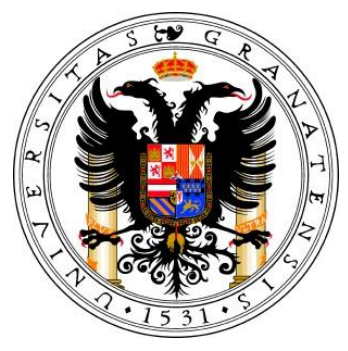

\title{
Maghemite Functionalization For Antitumor Drug Vehiculization
}

Programa Oficial de Doctorado en Física y Ciencias del Espacio

\section{TESIS DOCTORAL \\ Katarzyna Rudzka}

Departamento de Física Aplicada

Grupo de Física de Interfases y Sistemas Colloidales

Universidad de Granada

2013 
Editor: Editorial de la Universidad de Granada Autor: Katarzyna Rudzka

D.L.: GR 118-2014

ISBN: 978-84-9028-690-6 

La doctoranda Katarzyna Rudzka y los directores de la Tesis: Dr. D. Ángel V. Delgado Mora, Catedratico de Física Aplicada de la Universidad de Granada y Dr. D. Julián López Viota Gallardo, investigador de la empresa Vircell Micro Biologist, Granada

GARANTIZAMOS,

al firmar esta Tesis doctoral, titulada

\section{MAGHEMITE FUNCTIONALIZATION FOR ANTITUMOR DRUG VEHICULIZATON}

que el trabajo ha sido realizado por la doctoranda bajo la dirreción de los directores de la Tesis y hasta donde nuestro conocimiento alcanza, en la realización del trabajo, se han respetado los derechos de otros autores a ser citados, cuando se han utilizado sus resultados o publicaciones.

Granada, 7 de Junio de 2013

Directores de la Tesis:

Dr. Ángel V. Delgado Mora

Dr. Julián López Viota Gallardo

La doctoranda

Katarzyna Rudzka 

Nothing in life is to be feared, it is only to be understood.

Marie Curie-Sklodowska 



\section{Contents}

1 Introduction 1

1.1 Motivation .................... 3

1.2 Objectives and work plan . . . . . . . . . 5

$2 \begin{array}{lll}2 & \text { Magnetic nanoparticles for cancer imaging and therapy } & \mathbf{7}\end{array}$

2.1 Cancer pathology . . . . . . . . . . . . . 9

2.2 Magnetic nanoparticles in biomedical fields . . . . . . . . 13

2.2 .1 Generalities . . . . . . . . . . . . . . 13

2.2 .2 Drug delivery . . . . . . . . . . . . 16

2.2 .3 Hyperthermia . . . . . . . . . . . . . . . . . 19

2.2.4 Magnetic resonance imaging (MRI) . . . . . . . . 22

2.2.5 Physicochemical factors determining the fate of nanopart-

icles in the body . . . . . . . . . . . 27

2.2.5.1 Geometry . . . . . . . . . . . 27

2.2.5.2 Surface charge . . . . . . . . . . 33

2.2.5.3 Surface thermodynamics . . . . . . . . 34

2.2.6 Pharmacokinetics, biodistribution and biological fate 34

2.2 .7 Toxicity and biocompatibility . . . . . . . . 38

2.2.7.1 General concepts . . . . . . . . . . . 38

2.2.7.2 Toxicity evaluation and existing data . . . 44

2.2.7.3 Minimizing toxicity . . . . . . . . . 47

3 Synthesis and Characterization $\quad 49$

3.1 Design of magnetic colloids . . . . . . . . . . . . . 51

3.1 .1 Synthesis strategies . . . . . . . . . . . . . 51

3.1.2 Stabilization methods . . . . . . . . . . . 52

3.2 Synthesis and morphological study . . . . . . . . . 55

3.2.1 Maghemite synthesis and its functionalization . . . 55

3.2 .2 Morphological study . . . . . . . . . . . . . . 60 
3.3 Structure and chemical composition _. . . . . . . . . 69

3.3.1 X-ray Powder Diffraction (XRD) . . . . . . . . 69

3.3.2 X-ray Photoelectron Spectroscopy (XPS) . . . . . 72

4 Electrokinetic properties $\quad \mathbf{7 5}$

4.1 Description of the electric double layer . . . . . . . . . 77

4.2 Electrokinetic phenomena. Zeta Potential . . . . . . . 80

4.3 Methodology . . . . . . . . . . . . . . . 83

4.4 Results and discussion . . . . . . . . . . . . 83

4.4.1 Effects of $\mathrm{pH}$ and ionic strength on the electrokinetic properties of the maghemite nanocomposites. Design I. 83

4.4 .2 Results for Design II . . . . . . . . . . . . . . 87

$\begin{array}{lll}5 & \text { Magnetic characteristics } & 91\end{array}$

5.1 Generalities . . . . . . . . . . . . . . . 93

5.1.1 Ferromagnetism and ferrimagnetism . . . . . . . 93

5.1 .2 Superparamagnetism . . . . . . . . . . . . . . 98

5.1.3 Biomedical implications . . . . . . . . . . . . . 99

5.2 The magnetic properties of maghemite and nanocomposites 100

5.3 Magnetic Resonance Imaging . . . . . . . . . . . . . . . . 103

6 Capacity for antitumor drug vehiculization and tumor cell $\begin{array}{ll}\text { elimination } & 107\end{array}$

6.1 Stability of nanoparticulate drug delivery systems . . . . . . 111

6.2 UV-Vis spectrophotometry evaluation . . . . . . . . . 113

6.2.1 Procedure for the experimental determination of the antitumor drug vehiculization . . . . . . . . . . . 113

6.2.2 Absorbance of anticancer drug solutions . . . . . . . 114

6.3 Antineoplastic agent incorporation . . . . . . . . . . . . 115

6.3.1 Spectrophotometric determination . . . . . . . 117

6.3 .2 Electrokinetic analysis . . . . . . . . . . . . . . . 120

6.4 In vitro doxorubicin drug release . . . . . . . . . . . . 123

6.4.1 Methodology . . . . . . . . . . . . . . . 123

6.4.2 Results and discussion . . . . . . . . . . . . . . 124

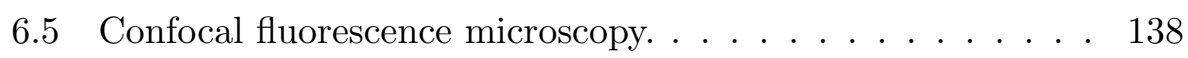

6.6 Cell viability studies . . . . . . . . . . . . . . . . . 138

6.7 Conclusions . . . . . . . . . . . . . . . . . . . . 144 
7 Resumen 145

7.1 Introducción. Objetivos . . . . . . . . . . . . . . 147

7.2 Síntesis y caracterización de núcleos magnéticos . . . . . . . 148

7.2.1 Estrategia de síntesis . . . . . . . . . . . . . . . 148

7.2 .2 Estudio morfológico . . . . . . . . . . . . . 150

7.3 Propiedades Electrocinéticas . . . . . . . . . . . . . . 154

7.3.1 Resultados: diseño I . . . . . . . . . . . . . . . . . . 154

7.3.2 Resultados: diseño II . . . . . . . . . . . . . . . . 155

7.4 Capacidad de adsorción de doxorrubicina . . . . . . . 158

7.4.1 Aspectos generales . . . . . . . . . . . . . 158

7.4.2 Determinación espectrofotométrica . . . . . . . . 158

7.4.3 Incorporación de agente antineoplásico . . . . . . . . 159

7.4.4 Análisis electrocinético . . . . . . . . . . . . . . 163

7.5 Liberación in vitro . . . . . . . . . . . . . . . . 163

7.5.1 Metodología . . . . . . . . . . . . . 163

7.5.2 Resultados . . . . . . . . . . . . . 163

7.6 Cultivos celulares . . . . . . . . . . . . . . 167

7.6.1 Metodología. . . . . . . . . . . . . 167

7.6 .2 Resultados . . . . . . . . . . . . 167

8 Conclusions 173 



\section{Chapter 1}

\section{Introduction}

In this chapter we describe the motivation of the research work performed as well as the objectives and work plan developed. We intend to give the reader the fundamentals of the necessity of carrying out investigations as those here described. 



\subsection{Motivation}

Fighting cancer has become an essential target of the scientific activity and health systems in all modern, socially concerned countries. The approaches are of course various, and the most recent advances deal with smart drug delivery systems and more selectivity in the transport of cancer drugs to tumor cells (Kim et al., 2012; Nakamura et al., 2012; Senter \& Sievers, 2012), but the contributions of physicochemical investigations must focus on some sort of "complementary" or "parallel" fields, notably the design of nanostructures aimed at transporting the therapeutic agent to the place of action, and releasing it at controlled rate.

An ideal drug-delivery system may be characterized by two distinctive features: the capacity to target and to control the cytotoxic agent release. Targeting will assure high efficiency of the drug and minimize the side effects. Chemotherapeutic substances, which are the pharmacological ground for approaches to cancer therapy, usually exhibit high cytotoxic properties, but they are not specific in reaching the biological target. This, in practice, results in a systemic distribution of the cytotoxic agents, which provoke well known side effects caused by unfortunate and undesirable interactions of the cytotoxic agent with healthy tissues (Dobson, 2006b; Sun et al., 2008). The reduction or prevention of side effects may also be obtained with the help of controlled release. Nanoparticulate drug delivery systems yield a better diffusion of the nanoparticles inside the body as their size enables delivery via intravenous injection or other ways. The nanoscale particle diameter of this kind of systems diminishes also the irritant reactions at the injection location. Initial attempts to guide treatment to a specific series of cells involved linking radioactive substances to antibodies specific to markers adsorbed on the surface of tumor cells. These strategies have provided some good results, and nanoparticulate drug delivery systems have demonstrated a lot of potential in the area (Thassu et al., 2007).

The idea of utilizing magnetic particles as a guide, applying an implanted permanent magnet or an externally applied field, to raise the accumulation of antitumor drugs to diseased parts of body dates back to the late 1970s. Widder, Senyi and colleagues (Widder et al., 1979; Senyei et al., 1978) reported the first preclinical experiments exploiting magnetic albu-

min microspheres loaded with doxorubicin in rats. However, the idea of taking advantage of iron oxide nanoparticles in biomedical and clinical ap- 
plications is not only because of their magnetic features but also thanks to their enormous potential in great number of biomedical and in vivo clinical applications, like magnetic resonance imaging (MRI) (Schlorf et al., 2011), magnetic hyperthermia treatment, tissue repair, contrast enhancement and gene (McBain et al., 2008) and/or drug delivery (Gupta et al., 2007; Viota et al., 2011). Moreover, it has been published that superparamagnetic iron oxide nanoparticles (SPIONs) are able to improve the efficiency of antitumor drugs, as well as to reverse multidrug resistance, and thus be employed as targeted drug carriers (Lin et al., 2007; Schroeder et al., 1998). Iron oxide nanoparticles can be produced with surface modification in order to make them more biocompatible. What is more, they can be attached to appropriate ligands which will serve as target specific receptors of various cancer cells for achieving targeted delivery systems (Pankhurst et al., 2003).

Drug delivery, hyperthermia and in vivo applications in imaging seem to be the most promising applications of SPIONs in the near future. However it should be underlined that knowing the safe upper limit of the safe use of SPIONs is of great importance (Gupta \& Curtis, 2004; Sonvico et al., 2005). Since their discovery, nanoparticulate drug-delivery systems based on magnetic iron oxide nanoparticles have become an attractive and challenging field of research (Berry \& Curtis, 2003; Pankhurst et al., 2003), but even though there are fascinating changes happening almost every day, there is still much uncertainty and issues regarding toxicity (Mahmoudi et al., 2011a; Laurent et al., 2011), biocompatibility or targeting efficiency and accumulation in RES (reticuloendothelial system) to be explained (Sharifi et al., 2012).

The experience of our group in the investigation of dispersed systems in which the suspended material consists of solid particles in the microto nanometer size range and the dispersion medium is an aqueous solution offers many possibilities for application in the field of drug transport and release, in which a number of contributions have already been published (Rudzka et al., 2012; Viota et al., 2011, 2013). In particular, we have recently (Duran et al., 2008) focussed on the design of magnetic drug delivery vehicles (MDDVs), based on a technology (that of magnetic colloidal vehicles) which started its development during the mid 1940's in the context of waste water treatment (Urbain \& Steman, 1941). New applications were soon envisaged, including enzimatic immobilization, magnetic separation of biomolecules, cell selection in a population, or biosensors (Pankhurst et al., 2003). Their use as drug delivery systems has been favored by the well known secondary effects of the anticancer drugs, associated to associated 
to their low specificity. In the present work, we intend to explore the possibilities of using magnetic drug vehicles (MDVs) as a possible technology capable of overcoming, at least in part, such difficulties.

\subsection{Objectives and work plan}

We have tried to find a research niche where new features could be added to the already large number of contributions dealing with the use of magnetic nanostructures in cancer treatment. In this sense, we have focussed on new designs of the nanostructures, as well as on possible drugs which could sit on them. With this idea, the following objectives and work steps are proposed in this Thesis:

- Synthesis and characterization of the magnetic nanoparticles: maghemite will be tested as magnetic core for the particles designed to be applied in the cancer field. The nanoparticles produced will be analyzed in relation to their size, shape, chemical composition, crystal structure, and magnetization.

- Silica coating of the magnetic nanoparticles. As a drug carrier, the system should be harmless to the body and excreted easily by it. Silica is a good candidate for incorporation in a drug delivery matrix due to its biocompatibility and has been used in many biomedical applications (Arruebo et al., 2006; Barbé et al., 2004; Slowing et al., 2007, 2008; Zhao et al., 2005). Silica is stable in water and easy to funcionalize. Another interesting benefit of this material is that it is degradable in an aqueous solution, so that problems related to the removal of the material after use can be avoided.

- Preparation of the particles for receiving their external gold layer. This is also a new approach in MDDV design: it has been shown that both in diagnostic and in therapeutic aspects, gold coatings offer spectacular possibilities (Nagahara et al., 2009). Because of the powerful interactions which exist between the gold surface and thiol/amineincluding molecules the surface of gold nanoparticles may be readily changed (Bhattacharya et al., 2007). In our case, we will follow two routes, one based on a previous coating with two different polyelectrolytes (PSS and PDADMAC), and the other on the application of an APTMS ((3-aminopropyl)trimethoxysilane) layer.

- Formation of the maghemite/gold complexes. By successive nucleations of gold in solution on the nanoparticles, it will be possible to 
obtain two kinds of gold-terminated nanostructures. Gold nanostructures present an interesting option for drug delivery (Connor et al., 2005; Ghosh et al., 2008), and have recently emerged as an attractive candidate for delivery of various payloads into their targets (Paciotti et al., 2006; Lopez-Viota et al., 2009; Liu et al., 2011). They will also be characterized by microscopy and magnetic response, and, additionally, by UV-Vis spectroscopy, since the presence of gold nanoparticles makes it possible absorption of radiation in the visible region because of surface plasmon resonance (Kelly et al., 2003). Not only does gold stabilize the particles by preventing their aggregation, but it also reduces considerably the toxicity of the nanocompounds (Liu et al., 2011). Even more, the capacity of the particles for absorbing electromagnetic radiation in the visible and IR regions of the spectrum, opens the possibility of using them as agents for electromagnetic (in addition to magnetic) hyperthermia (Govorov \& Richardson, 2007; Prashant et al., 2007).

- Drug transport. This subject can be considered as the true core of our work. The drug used to test the loading and release capacities of the nanostructures designed will be doxorubicin, a cationic drug, of routine use in the treatment of many solid tumors, including lymphoma, osteosarcoma, Kaposi 's sarcoma, Hodgkin and non-Hodgkin lymphoma and soft tissue sarcomas (Minotti et al., 2004). The adsorption density achieved, as well as the release rate, will be evaluated.

- Magnetic resonance imaging (MRI). It is known that magnetic nanoparticles can enhance the contrast in MRI because of their high transversal relaxivity. It will be a significant and original contribution of the present work to evaluate the magnetic relaxivities of our nanostructures and compare them to those presently existing in the market. 


\section{Chapter 2}

\section{Magnetic nanoparticles for cancer imaging and therapy}

In this chapter we describe various ways of magnetic NPs synthesis and their physicochemical characterization. We will also consider their biodistribution, pharmacokinetics and toxicity. Biomedical applications will be an issue of special importance. 



\subsection{Cancer pathology}

Cancer is a generic group of specific illnesses that can affect various parts of the body. According to the World Cancer Report, in 2008 there were over 12 million of estimated global cancer burden, 25 million of people alive with cancer and 7 million deaths because of this disease. In addition, it is worth to mention that the number of cancer deaths is expected to rise up to 17 million by 2030 (World Health Organization, 2008); cancer occurs due to mutations in genes which govern cell cycles (Husband \& Reznek, 2000), invasion of adjacent tissue, metastasis (dissemination to other parts of the body), immortality (self-protection against programmed cell death). Almost all cancer cells present irregular cell-cycle control. When the tumor has become bigger than ca. 1-2 mm, it starts to create new blood vessels (angiogenesis) because of substances liberated into the tumor microenvironment that stimulate it. Malignant vessels are irregular, showing spaces between endothelial cells, break of the basement membrane, and no muscle without bumps inside the capillary walls (Husband \& Reznek, 2000). They present a chaotic structure, are winding and dilated, show variable diameter and excess branching, and possess a vasculature characterized by disorder (Brigger et al., 2002). By reason of the walls, the vessels are characterized by many gaps, like for example endothelial fenestrae, vesicles and transcellular holes and a basement that is not continuous. These elements make the capillaries more permeable than healthy ones, permitting substances to enter more readily and create the surrounding environment (Cheon \& Lee, 2008). Vascular permeability is strongly determined by the type of cancer and the organs which affects.

Tumor Angiogenesis. The rate of tumor growth is determined by the diffusion of oxygen and nutrients and normally it begins as a cluster of cells that can be benign or malignant (Fig. 2.1). When the size of the tumor is greater than 1-2 mm, the interior cells are not able to grow and proliferate due to a scarcity of nourishing substances and turns into dormant or necrotic, resulting in the creation of a three-layered structure: a center with dead cells encircled with a first layer of quiescent cells and the outer thin layer containing proliferating cells (Kerbel, 2000). The tumor can stay on in a dormancy state while balance exists between cell death and cell proliferation. Simply by creation of new blood vessels the tumor 


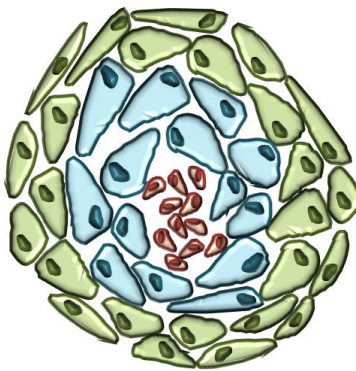

Benign tumor

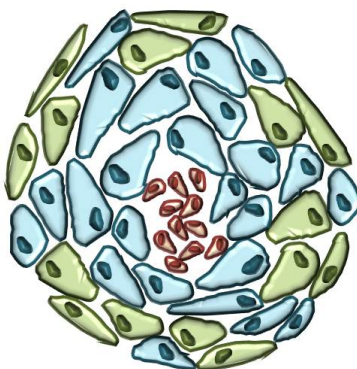

Malignant tumor

Figure 2.1: Schema of two types of tumors. In the case of a malignant one, mutated (blue) cells are combined with normal ones, trying to attack the surroundings tissues. In benign tumor, mutated (blue) cells are present inside a single group separated from normal (green) cells by a clear border. Note that the inner cells (red) are necrosed/dead cells.

can come out of the dormancy state. The fundamental events leading to the tumor vessels growth and their sprouting are analogous to those existing in normal tissue angiogenesis (Fig. 2.2). In case of tumor angiogenesis, there are three significant occurrences that have to happen for creation of new blood vessels: disintegration of the basement membrane, movement of endothelial cells, and proliferation to yield new cells for vessel growth (Hobson \& Denekamp, 1984). However, contrary to physiological angiogenesis, the angiogenesis switch continue to be turned on as a result of the persistent manufacturing of angiogenic factors inside the tumor areas that are not sufficiently oxygenated. Constant growth of new capillaries within the tumor results in an imperfect, tortuous, non-mature and leaky vasculature network which is deficient in a stable basement membrane (Plank \& Sleeman, 2003).

The enhanced permeability and retention (EPR) effect. Tumor tissues exhibit many abnormalities which provoke excess leakage of blood plasma constituents and other different macromolecules (Lyer et al., 2006). The important permeability to macromolecules of blood vessels within the tumor in comparison to normal blood vessels, and the weaker clearance of these macromolecules from the tumor interstitial tissues (provoking longer retention of various molecules), is called EPR effect (enhanced permeability and retention effect) (Fig. 2.3) (Greish, 2007). The EPR effect was first described by Maeda (Maeda et al., 1986). As a result of this effect, bigger 


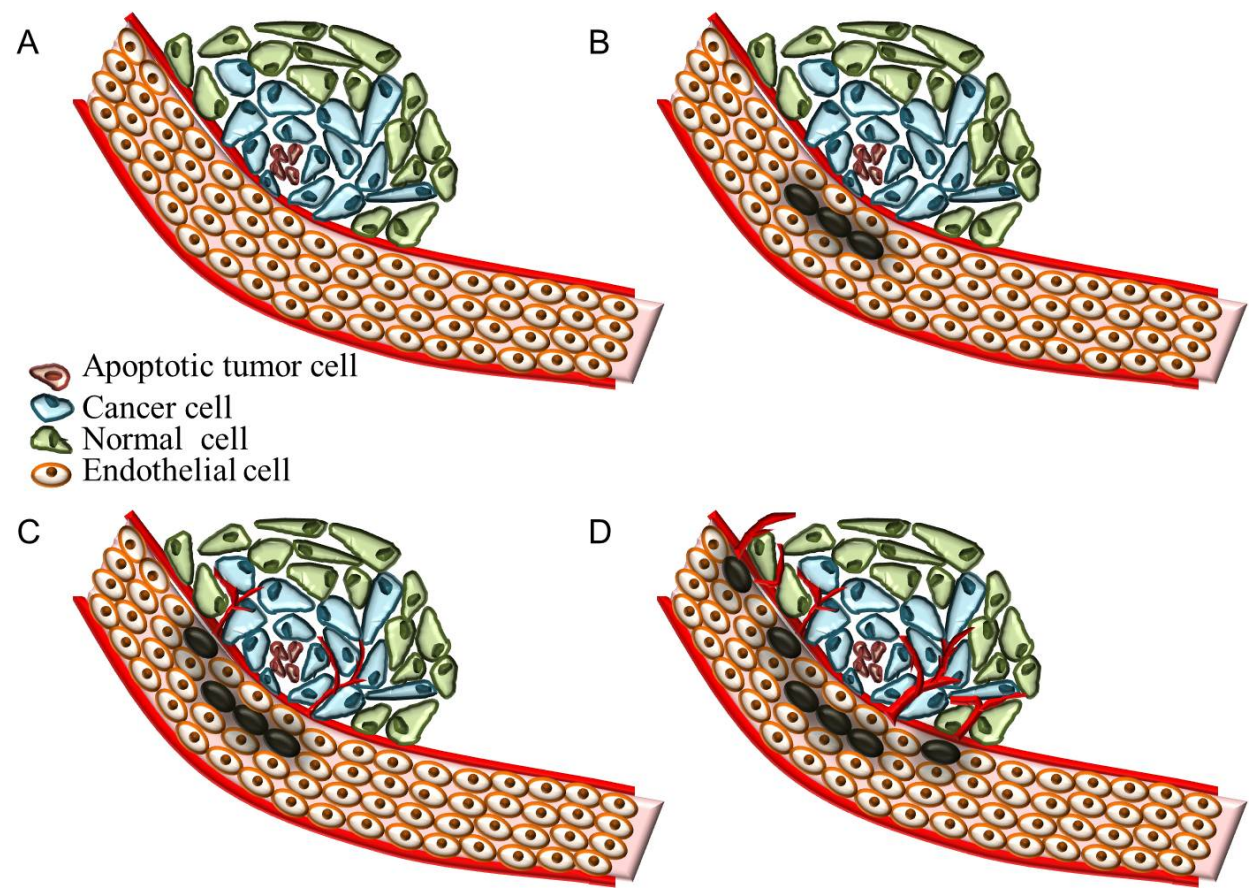

Figure 2.2: The fundamental events occurring in tumor angiogenesis: A) tumor cluster next to the blood vessel in a dormancy state, B) dissolution of the basement membrane (black points) provoked by the angiogenic switch being turned on, resulting in migration and proliferation of endothelial cells which lead to new blood vessels formation C) and D). 


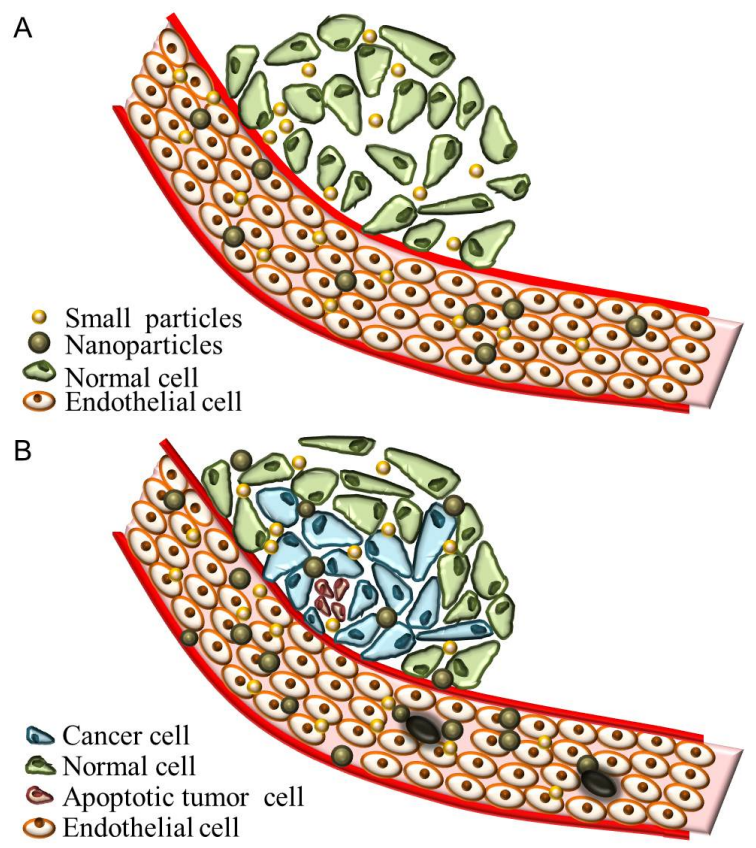

Figure 2.3: The enhanced permeability and retention (EPR) effect. A- Normal/Healthy tissues where only small molecules can pass across blood vessel walls; B- Permeable blood vessels within the tumor where nanoparticles and other macromolecules can spread out within the tissue because of the leaky arrangement of blood vessels.

molecules can gather within the tumor in much larger amounts in a comparison to normal tissues within 1-2 days (Maeda, 2001). The EPR effect can be employed to accomplish passive targeting of tumors for drug delivery and imaging by working with nanoparticles (NPs) and macromolecules in place of traditional small-molecule drugs. For the purpose of efficiently exploiting the EPR effect for disease detection as well as cancer treatment, the molecular weight of a drug should be more than $40 \mathrm{kDa}$ (Maeda et al., 2009). Cut-off sizes for pores in cases of various tumors are in the range between 380 and $780 \mathrm{~nm}$ (Brigger et al., 2002; Williams et al., 2009). 


\subsection{Magnetic nanoparticles in biomedical fields}

\subsubsection{Generalities}

Nowadays a lot of magnetic materials are studied with the intention of using them in drug delivery and more particularly in cancer therapy. One of the most important benefits which they present is targeting drug delivery to the tumor area. There are two ways under which we can deliver a cytostatic drug to the tumor area; this may be obtained either by passive or active targeting (See Fig. 2.4) (Parveen et al., 2012).

Passive targeting and EPR effect. Passive targeting takes advantage of the anatomical dissimilarities between normal and tumor tissues in order to transport the therapeutic drug to the desired area, since the functions and activity of non-healthy tissues can be modified by way of the EPR effect.

A great number of studies has been performed on passive drug targeting. One of the examples is the commercially available Doxil (Caelyx) which is a PEG-coated liposome with doxorubicin loaded as a therapeutic drug. This product exhibits good doxorubicin retention with increased time within circulatory system, and moreover it shows that its efficacy is six times higher than that of free therapeutic drug. It has been authorized for the therapy of ovarian cancer, metastatic breast cancer and Kaposi's sarcoma (Gullotti $\&$ Yeo, 2009). The tumor-targeting capability of chitosan nanocompounds with glycol coating and cisplatin loading has been as well demonstrated in in vivo studies. It has been reported that nanocompounds were gathered within tumor site in mice due to the EPR effect and extended time in the circulatory system (Parveen et al., 2012).

Active targeting. The principal physiological property of cancer cells is their intensified metabolic velocity. A tumor which is increasing in size and expanding quickly, needs different nutrients and vitamins. As a consequence, tumor cells overexpress a large number of specific receptors, that may be employed as objects to carry cytotoxic agents to the tumor. Generally, a tumor-targeting drug delivery system is made up of a tumor identification part and a cytotoxic drug linked directly or through a proper linker in order to construct a conjugate. These tumor-targeting unions carrying the cytotoxic agent may be categorized into various groups depending on the class of cancer recognition half, which can include by folic acid, monoclonal 


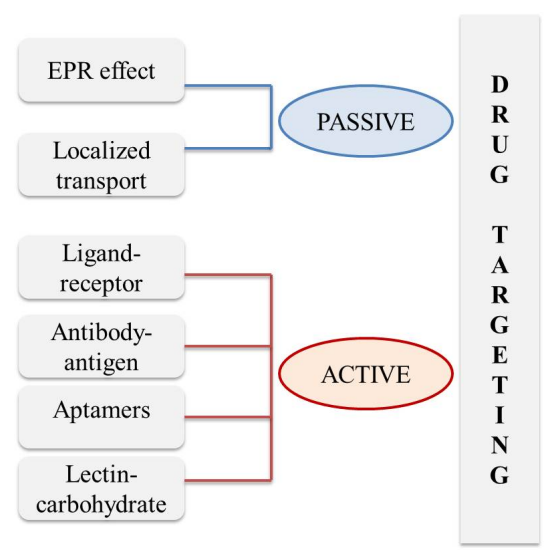

Figure 2.4: Schematic illustration of distinct methods for targeting drug delivery (Parveen et al., 2012).

antibodies, hyaluronic acid, polyunsaturated fatty acids and oligopeptides (Jaracz et al., 2005).

The effectiveness of antibody-drug conjugates employed as antitumor drugs is considerably conditioned by the tumor specificity of antibodies, the strength of the antitumor drug, and the efficacy of the linker. The antitumor drug in the conjugate must be very potent. This is due to the fact that just a small amount of molecules may be loaded on every antibody part without reducing the attachment affinity of the antibody (Chari, 1998). Another argument for the need of employing strong antitumor agents is that there exists just a limited number of antiagents overexpressed on the surface of malignant cells.

Besides the antibody and the antitumor drug parts, the linker component is also of great importance. An excellent linker has to be stable within the circulation system and at the same time has to be adequately attached to the malignant cells. The most largely used linkers can be classified into three types: i) hydrazone; ii) peptide; iii) disulfide. The choice of the linker is determined by the cancer type and the antitumor drug needed. None of these linkers is for the general use and all of them possess their pros and cons.

Tumor-targeting with folic acid. Folic acid is a member of the vitamin B family. It is significant in the creation of new cells because it plays a role in the biosynthesis of nucleotide bases. There exist two folic acid receptors which are connected to membranes, namely FR- $\alpha$ and FR- $\beta$. Both of them link to folic acid with great affinity (Elnakat \& Ratnam, 2004). In 
healthy tissues the expression of FR is insignificant and limited to different types of epithelial cells (placenta, lungs or kidneys), whereas FR is found everexpressed in various tumors (ovarian and endometrial cancers). The radiolabeling analysis has demonstrated that there is 20 times more folic acid bound to the tumor cells than to healthy epithelial cells (Weitman et al., 1992).

Few studies have been published on conjugates bearing folic acid and antitumor agent (e.g. paclitaxel, maytansine or mitomycin C) which are characterized by low molecular weight. One of this examples in the paclitaxel bound to folic acid via an oligoethyleneglycol linker (Lee et al., 2002). These conjugates exhibit much higher cytotoxicity than paclitaxel alone. In a recent study, nanoparticles of polyactide with paclitaxel loading and conjugated with aptamer showed improved targeting to the tumor site (Tong et al., 2010). Other studies have shown that different tumor cell lines (liver, colon, and breast), that overexpress at different degrees the three types of folic acid receptors, can be selectively targeted by functionalizing magnetic nanostructures with a final layer of folic acid (Viota et al., 2013).

Taking into consideration that one liposome is able to bear a drug cargo of approximately $10^{3}-10^{4}$ molecules, liposomal conjugates provide chance to create effective targeted drug delivery systems. In one of the studies performed in this direction, folic acid was bound to Doxil (Vaage et al., 1992). In vitro studies showed that cytoxicity of liposomal formulation with attached folic acid was 10 times higher than that of Doxil, however the cytotoxicity value of the conjugate was equal to that of doxorubicin alone (not encapsulated into the liposome).

Tumor-targeting with hyaluronic acid. Hyaluronic acid (HA) is a linear and negative polysaccharide, which consists of two alternating parts. It plays an important role in different functions such as cell growth, differentiation and migration (Toole, 1982).

It has been demonstrated that the HA level is raised in different cancer cells (Toole et al., 2002). This probably is responsible for less dense matrix formation, thus raising cell capacity to move in addition to enhance intrusive ability within other tissues (Yang et al., 1993). It is worth to mention that different tumors (e.g. epithelial, ovarian, colon, stomach) overexpress HA-linking receptors CD44 and RHAMM (Day \& Prestwich, 2002; Turley et al., 1993). As a result, these malignant cells exhibit raised attachment and internalization of HA.

Tumor-targeting with peptides. Peptide-based targeted drug delivery is characterized by a great potential for tumor-specific drug delivery. An obvi- 
ous profit of this technique is an outstanding likelihood that tumor-specific peptide sequences for different cancers may be found by screening proper combinatorial libraries. Because of the fact that majority of gastrointestinal cancers are hard to treat as they frequently present multidrug-resistance, this method may help in the progression of effective chemotherapy.

Interest of magnetic particles. In the particular case of magnetic nanoparticles, it is clear that they can contribute nowadays in biomedicine, starting from their unique size which place them in a world of cells (10 $100 \mu \mathrm{m})$ and viruses $(20-450 \mathrm{~nm})$ or even proteins $(5-50 \mathrm{~nm})$ and genes $(2 \mathrm{~nm}$ in width and a length of $10-100 \mathrm{~nm}$ ). The second important fact is that they can be controlled by means of an external magnetic field gradient. This feature opens up lots of applications, like magnetic targeting or transporting and/or immobilization of biomolecules. The third reason for their importance is that magnetic nanocompounds are able to resonantly respond to an applied magnetic field which is varying with time. As a result, energy will be moved from the exciting magnetic field to the nanoparticle. These, and many other potential applications, are now present and available in medicine and biology thanks to the basic physical properties of magnetic nanocomposites (Pankhurst et al., 2003).

The biomedical applications of the nanoparticles requires the following characteristics: i) large magnetic moments (the larger the magnetic moment the lower the amount of magnetic nanoparticles needed); ii) biocompatibility (an equilibrium between a magnetic moment long enough and biocompatibility should be maintained) (Sandhu et al., 2010); iii) size (the perfect size ranges between 10 and $\sim 70 \mathrm{~nm}$ critical single-domain size).

As mentioned, with the aim of increasing the biocompatibility (among other features) of the nanoparticles, a polymeric coating of the surface of the nanoparticles has to be performed. However, this modification can reduce the magnetic characteristics of the final composite nanoparticles. Generally, an important decrease can be found in the literature in the saturation magnetization value (Fu et al., 2001), as a result of the non-collinear spin structure on the particle-coating interface. Nevertheless, it is worth to mention that this reduction depends on the type of coating.

\subsubsection{Drug delivery}

The main drawback of the majority of available chemotherapies is that they are comparatively non-specific. The intravenous administration of the 
drugs gives rise to an overall distribution in the body, producing harmful side-effects due to the non selectivity between tumor and healthy cells.

The awareness of this led researchers in the late 1970s to propose exploiting magnetic carriers in order to reach specific sites inside the body (Widder et al., 1978; Senyei et al., 1978; Mosbach \& Schröder, 1979). The goals are the following:

(i) reduction of the overall drug distribution within the body which leads to the diminution of the side-effects;

(ii) reduction of the required dose because of a more effective and localized targeting of the administered therapeutic drug.

In the case of therapies which are targeted by magnetic field, the therapeutic drug is connected to a biocompatible magnetic nanoparticles, that plays the role of a carrier. As these complexes go into the bloodstream, an external magnetic field is applied in order to concentrate them at the chosen targeted site inside the body. At the moment that the drug/carrier unit becomes concentrated at the target site, the therapeutic drug can be liberated by changes in the physiological conditions ( $\mathrm{pH}$, osmolality, temperature) or via enzymatic way, and in this manner can be taken up by the malignant cells.

The physical bases that underlie magnetic targeting is the magnetic force exercised on superparamagnetic particles by means of magnetic field gradient. The therapy results effective if physical parameters like the field strength and gradient, and the volumetric and magnetic properties of the particles are well controlled. Hydrodynamic parameters (blood flow rate, particle concentration, infusion rate or circulation time) also play an important role as the carriers are administered to the patient by intravenous or intra-arterial way. Physiological factors like reversibility, volume of tumor, binding strength of the complex and the depth of tissue until reaching the target site are not less significant (Lübbe et al., 1999).

In the majority of cases it is a strong permanent magnet set outside the body above the target site which produces the magnetic field gradient. Preliminary studies indicate that targeting is likely to be most effective in areas with slower blood flow, especially when the target site is near the magnet (Voltairas et al., 2002). Moreover, there are mathematical models created in order to find out trajectories of the particles for different field/particle configurations in 2D, which also include the factor of their movement when they are coming nearer to the vessel wall (Cummings et al., 2000). This fact is important as close to the walls the movement of the particle is no 
longer controlled by Stoke's law (for the drag force), and parameters of the hydrodynamics are changed.

As mentioned, the first time magnetic particles were utilized as carriers was for delivery of doxorubicin (antitumor drug) to sarcoma tumor which was implanted in rats (Widder et al., 1983). Different magnetizable nanoparticles (e.g. cobalt, maghemite, etc.) have been tested with various polymer coatings, like PLA, PLGA, dextran or chitosan, and these particular nanocompounds may be employed for targeted drug delivery.

Some researches have shown application of magnetic colloids for drug delivery purposes. New hybrid magnetic nanoparticles smaller than $160 \mathrm{~nm}$ made of hyaluronic acid (HA) and iron oxide have been prepared. These nanostructures were later examined as for their capacity for distributing peptides to the cells by means of HEK293 and A549 cells. The data obtained demonstrate that the nanocomposites carried peptides to the cells to a $100 \%$ extent, and that these nanoparticles could be utilized in creating efficient tissue and cell targeting systems (Kumar et al., 2007). Other research groups investigated highly magnetic nanoparticles with biodegradable polymer coating also for applications in biology and medicine. The nanocompounds synthesized presented good encapsulation capability as well as high magnetic response (magnetization values in the range of $26-40 \mathrm{emu} / \mathrm{g}$ ) due to a fact of possessing a lot of magnetic material (even up to $60 \%$ ) (Liu et al., 2007a). Water-dispersible nanospheres of iron oxide with coating of oleic acid and Pluronic were also investigated as drug delivery systems. It was demonstrated that they were able of delivering high amounts of anticancer drugs. Data showed the continuous release process of antitumor drug, doxorubicin, during at least 2 weeks and the nanospheres showed also a continuous keeping of drug within cells compared to doxorubicin in solution. Experiments illustrated also an antiproliferative effect determined by the drug dose, for cases of breast and prostate cancer cell lines (Jain et al., 2005).

In the recent past, drug/gene delivery systems for brain tumors have been created based on magnetic iron oxide particles with PEI coating. As this study demonstrates, when drug-loaded nanospheres were administrated in the carotid artery with combination of magnetic targeting, an increase of 30 times in tumor capture of nanospheres was observed as compared to intravenous administration (Chertok et al., 2010). In another interesting investigation functionalized dendrimers which may in effect pass over the $\mathrm{BBB}$ and concentrate within malignant glioma cells have been prepared. It was determined that dendrimers with sizes smaller than $11.7-11.9 \mathrm{~nm}$, 
which were delivered to the patient in a intravenous way, were capable of crossing pores of the blood-brain tumor barrier, and bigger dendrimers could not do it (Sarin et al., 2008).

There are some problems that may exist in magnetically targeted drug delivery. These limits/restrictions are the following: (i) the probability that the blood vessels will embolize in the target site because of collection of the magnetic carriers, (ii) problems in upscaling from the model used in animals as a result of larger distances connecting the target region and the magnetic carrier, (iii) after the drug is released, it is obviously out of the influence of the field, and (iv) magnetic particles may induce due toxic reactions. However, it is demonstrated that these limitations can be overcome and safety problems resolved.

\subsubsection{Hyperthermia}

This phenomenon includes dispersing magnetic nanoparticles all through the target site, and next applying an external magnetic field with strength and frequency high enough to make the particle temperature raise, and, if the temperature can be kept up over the therapeutic limit of $42^{\circ} \mathrm{C}$ during 30 min or more, the tumor tissues are destroyed. Although almost all of the hyperthermia tools have limited use due to unwanted simultaneous heating of the healthy tissues, hyperthermia of magnetic nanoparticles is of great interest since it can offer a method to make certain that nothing but the planned tumor tissues are heated. Appropriate tumor entities must be scrupulously chosen for existing oncological concepts, i.e. tumors with serious predictions and not easy to heat up, like brain tumors or those in importantly perfused organs (e.g. lung, liver or kidney). The difficulty of this technique is the ability to deliver/transport a satisfactory amount of the magnetic nanoparticles so that sufficient heat production occurs in the target site, employing AC magnetic field conditions which are admitted clinically.

Numerous studies have proved the efficacy of this therapy in animal models (Moroz et al., 2002) and there have been presented reports of the satisfactory application of this method in investigations on cells and human tissues (Chan et al., 1993; Jordan et al., 1996, 1997). As a result, hyperthermia has been accepted by the FDA to utilize it alone or in combination with radiation in cases of some solid malignant tumors which are present onto and under the surface of the body (e.g. sarcoma, adenocarcinoma, melanoma). Clinical trials employing combined therapy have revealed that 
$83.7 \%$ of cancer patients experience a considerable reduction of tumor mass, and $37.4 \%$ of these patients had a total tumor regression. Nowadays there are companies (e.g. Aspen MediSys or MagForce) which are working in methods that produce heat thanks to exposure of the magnetic nanoparticles to magnetic fields (Colombo et al., 2012).

It is essential to understand the physical basis through which heat generation in magnetic nanoparticles occurs via AC magnetic fields. Estimating the heat deposition rate which is needed to reach the target temperature is complicated because of the existence of blood flow and tissue perfusion, both of which are dominating factors in tissue cooling and also differ energetically when tissue is made hot. As a rule of thumb, which is utilized in many cases, a heat deposition rate of $100 \mathrm{mWcm}^{-3}$ of tissue will be enough in most situations.

The strength and frequency of the AC magnetic field employed to produce the heating is restricted by damaging physiological reactions to magnetic fields of high frequency (Reilly, 1992). They include stimulation of peripheral and skeletal muscles, cardiac stimulation and arrhythmia as well as non-specific inductive tissue heating. Frequencies $(f)$ utilized normally are set between 0.05 and $1.2 \mathrm{MHz}$ and amplitudes employed are in a range of $\mathbf{H}=0-15 \mathrm{kAm}^{-1}$. It has been demonstrated that when $\mathbf{H} \cdot f$ is not greater than $4.85 \times 10^{8} \mathrm{~A} \mathrm{~m}^{-1} \mathrm{~s}^{-1}$ the field is free from harm (Atkinson et al., 1984).

The amount of magnetic particles which will be enough to generate the required needed temperature is determined largely by the method of administration. A realistic supposition is that ca. 5-10 $\mathrm{mg}$ of magnetic nanoparticles concentrated in every $\mathrm{cm}^{3}$ of tumor tissue is suitable for hyperthermia in human patients.

Heating mechanisms. Ferro- or ferrimagnetic particles (FM) undergo magnetic hysteresis when there is a time-varying magnetic field applied and this results in magnetically induced heating (Fig. 2.5). The amount of heat produced per unit volume is the area of the M-B hysteresis loop multiplied by the frequency:

$$
P_{F M}=\mu_{0} f \oint \mathbf{H} d \mathbf{M}
$$

where $f$ is the frequency, $\mu_{0}$ represents the vacuum permeability, $\mathbf{H}$ and $\mathbf{M}$ represent the field strength and the magnetization, respectively.

In this equation other probable mechanisms for magnetically caused heating (like eddy current heating or ferromagnetic resonance) are neglected, as these are normally not relevant in these circumstances. 


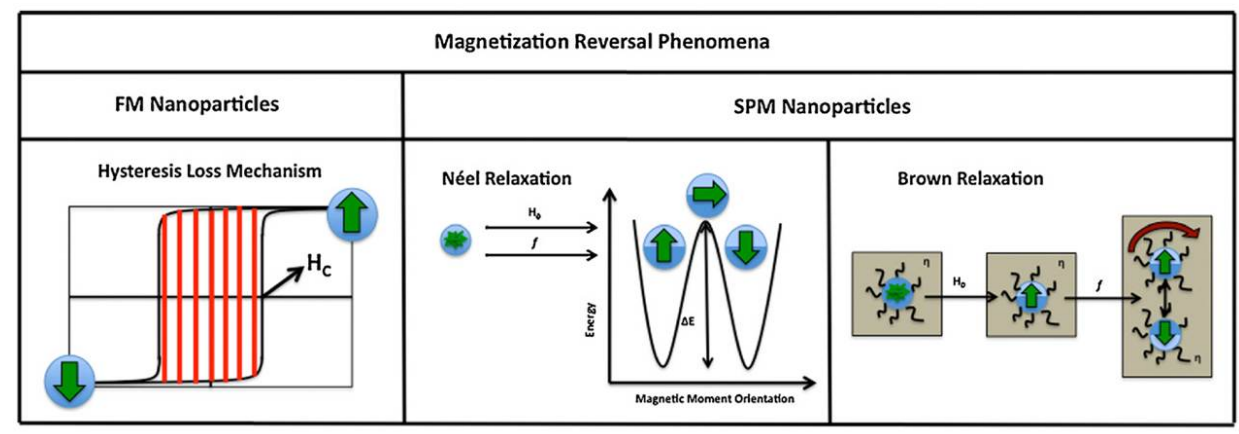

Figure 2.5: Magnetization reversal phenomena for heat generation under the effect of an alternating magnetic field $\left(\mathbf{H}_{0}, \mathbf{f}\right)$. Left: Hysteresis loss mechanism of heat generation by ferro- and ferrimagnetic nanoparticles; center: Néel- and right- Brown relaxation mechanisms of heat generation by superparamagnetic nanoparticles (Figuerola et al., 2010).

Theoretically, important hysteresis heating of the ferro- or/and ferrimagnetic particles may be achieved employing strongly anisotropic magnets like Nd-Fe-B or else Sm-Co; although the limits on $\mathbf{H}$ that may be utilized signifies that fully saturated loops cannot be employed. Minor loops (unsaturated ones) can be utilized, and would result in heating, but only at very reduced levels. Nevertheless, this could be obtained only with a group of uniaxial particles which are characterized by perfect alignment with $\mathrm{H}$. This configuration might be hard to achieve, if not absolutely impossible in vivo. The most realistic group of randomly aligned FM particles can be of $25 \%$ of the ideal maximum.

Nonetheless, in the last years the area of magnetic nanoparticles for hyperthermia treatment has been modernized because of the arrival of "magnetic fluid hyperthemia". Recall that a "magnetic fluid" or "ferrofluid" is a suspension consisting of superparamagnetic nanoparticles in water or else in a hydrocarbon fluid (Jordan, 2001). The magnetization of these ferrofluids while the magnetic field is not applied goes to zero because of thermal randomization of orientations. This means that hysteresis is not present, and other relaxation phenomena must be considered, namely, particle rotation inside the magnetic fluid, or atomic magnetic moments rotation inside each particle. The former is called "Brownian rotation" and the moment rotation inside each particle has been given the name of "Néel relaxation".

The physical principles of the process of heating of superparamagnetic nanoparticles by means of magnetic fields have been recently re-examined (Rosensweig, 2002). They follow the Debye model originally created for 
characterizing the dielectric dispersion in case of polar fluids (Debye, 1929). If we assume that there are very small interactions between component superparamagnetic nanoparticles, and that the field strength is small, the phase lag between the magnetization $\mathbf{M}$ and the field $\mathbf{H}$ will be represented by the imaginary component $\chi^{\prime \prime}$ of the magnetic susceptibility $\chi=\chi^{\prime}+i \chi^{\prime \prime}$. The heating power can be expressed as:

$$
P_{S P M}=\mu_{0} \pi f \chi^{\prime \prime} H^{2}
$$

Normally, measurments of the heat production from magnetic nanoparticles are estimated in terms of the specific absorption rate (SAR), expressed in $\mathrm{Wg}^{-1}$. The product of the particle density by the SAR gives $\mathrm{P}_{\mathrm{FM}}$ and $\mathrm{P}_{\mathrm{SPM}}$, and thus this value permits comparing the efficacies of different magnetic nanoparticles of any size. After comparing these parameters, it is obvious that for achieving a fully saturated loop the majority of FM particles need applied fields of ca $100 \mathrm{kA} \mathrm{m}^{-1}$ (in strength). Due to the constant value of $15 \mathrm{kA} \mathrm{m}^{-1}$ which has to be employed, only small hysteresis loops can be used, resulting in low specific absorption rate. On the contrary, superparamagnetic particles have ability to produce imposing/great amounts of heating lower fields. For instance, one of the published SAR values for SPM fluid is $45 \mathrm{Wg}^{-1}$ at $300 \mathrm{kHz}$ and $6.5 \mathrm{kA} \mathrm{m}^{-1}$, and can be extrapolated to $209 \mathrm{Wg}^{-1}$ for $14 \mathrm{kA} \mathrm{m}^{-1}$ (Hergt et al., 1998). For the same field strength $\left(14 \mathrm{kA} \mathrm{m}^{-1}\right)$ the best SAR value obtained for FM material is $75 \mathrm{Wg}^{-1}$.

\subsubsection{Magnetic resonance imaging (MRI)}

Magnetic resonance imaging (MRI) has become a tool of fundamental importance in the diagnosis of cancer (and other pathologies). It is based on the phenomenon of nuclear magnetic resonance, a manifestation of the existence of the small magnetic moment of the proton, and the fact that cells and tissues have a huge number of protons, so that the macroscopic response of the tissues under the action of magnetic field can be measured if the field is large enough. This is not the place for going into details (see, e.g., (Elster \& Burdette, 2001)); at this point it may suffice to keep in mind that although the number of protons feeling the field is rather small (only three out of $10^{6}$ proton magnetic moments, $\mathbf{m}$, are oriented parallel to the external field $\mathbf{B}_{\mathbf{0}}$, even if this is as high as $1 \mathrm{~T}$, the number of protons available is so great $\left(6.6 \times 10^{19}\right.$ in each $\mathrm{mm}^{3}$ of water $)$ that the productive signal $\left(2 \times 10^{14}\right.$ proton moments/ $\left.\mathrm{mm}^{3}\right)$ is noteworthy.

The principles of the method are shown in Fig. 2.6: the capture of the 


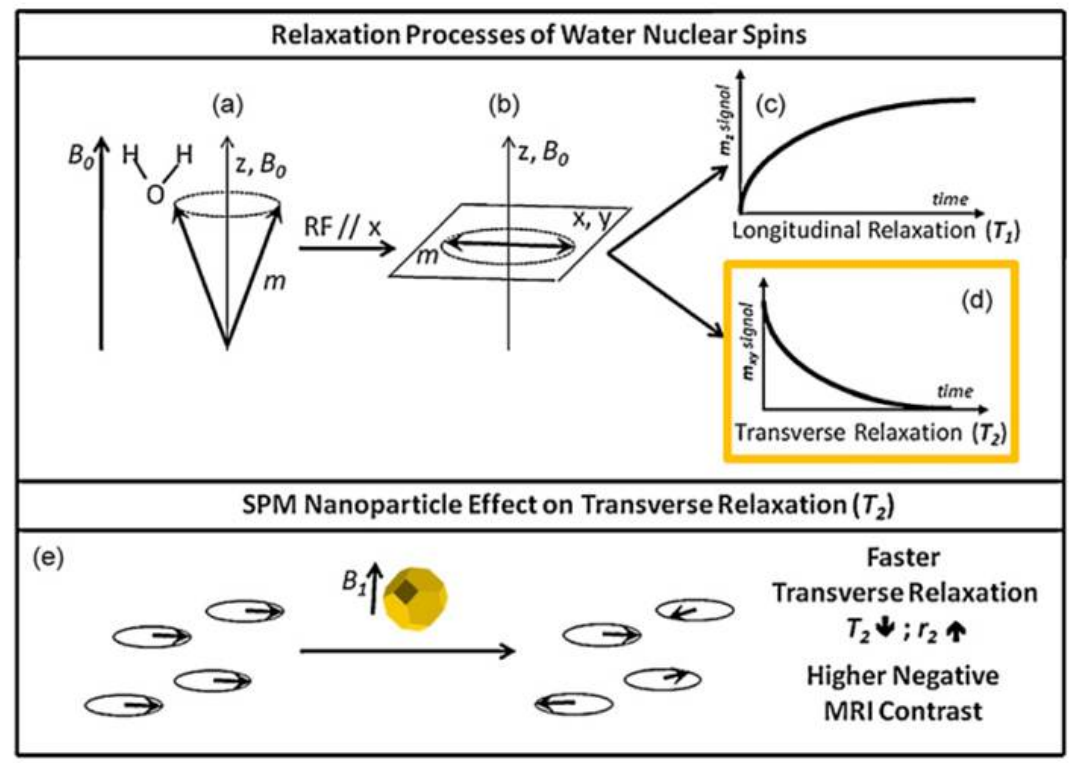

Figure 2.6: Illustration of the magnetic resonance (MR) principle and the role of magnetic nanoparticles as a contrast agent. Above: (a) net magnetic spins $(\mathbf{m})$ of water protons precess around the direction of the applied external magnetic field $\left(\mathbf{B}_{\mathbf{0}}\right)$; (b) upon application of an RF pulse, $\mathbf{m}$ begins precessing perpendicularly to $\mathbf{B}_{\mathbf{0}}$; (c) $\mathbf{m}$ relaxes back to its original equilibrium states through longitudinal $\left(\mathrm{T}_{1}\right)$ and $(\mathrm{d})$ transverse $\left(\mathrm{T}_{2}\right)$ modes; (e) in the presence of magnetic nanoparticles, the spins of the water protons start precessing non-homogeneously under the additional effect of the local dipolar field $\left(\mathbf{B}_{\mathbf{1}}\right)$ originated by the nanoparticles. Consequently they relax faster and induce a strong MRI signal, which produces strong MR contrast effects (Figuerola et al., 2010). 
signal is possible by resonant absorption: a rotating field perpendicular to $\mathbf{B}_{\mathbf{0}}$ is applied, the frequency of which is adjusted to the Larmor precession frequency of the magnetic moment to be determined, around the main field. In the case of the proton, the frequency equals $\omega_{0}=\gamma B_{0}$, where the gyromagnetic ratio $\gamma$ in case of ${ }^{1} \mathrm{H}$ protons is $\gamma=2.67 \times 10^{8} \mathrm{rad} \mathrm{s}^{-1} \mathrm{~T}^{-1}$. In this way when $\mathbf{B}_{0}$ is equal to $1 \mathrm{~T}$, the resonant radio-frequency equals $\omega_{0} / 2 \pi=42.57 \mathrm{MHz}$. In reality, the transverse field is applied as a series of pulses in the MRI scanner, and the relaxation of the signal absorption after switching off the pulse is measured by means of the evaluation of the current induced in sensing coils (Pankhurst et al., 2003). For the situation b) in Fig. 2.6, the relaxation signals take the form:

$$
\begin{gathered}
m_{z}=m\left(1-e^{-t / T_{1}}\right) \\
\text { and } \\
m_{x, y}=m \sin \left(\omega_{0} t+\phi\right) e^{-t / T_{2}},
\end{gathered}
$$

where $m$ is the magnetic moment to be determined, $\mathrm{T}_{1}$ represents the longitudinal (spin-lattice), $\mathrm{T}_{2}$ the transverse (spin-spin) relaxation times, and $\phi$ is a phase constant. The spin-spin relaxation is comparatively fast in comparison with the spin-lattice one, and it is impelled by the loss of phase coherence in the precessing protons because they interact magnetically with each other and with any other moments in the surrounding tissue. The loss of phase may be also influenced by regional lacks of homogeneity in the utilized spin-lattice field, resulting in the substitution of $\mathrm{T}_{2}$ in Eq. 2.4 for the (shorter) $\mathrm{T}_{2}^{*}$ relaxation time:

$$
\frac{1}{T_{2}^{*}}=\frac{1}{T_{2}}+\gamma \frac{\Delta B_{0}}{2}
$$

where $\Delta \mathbf{B}_{\mathbf{0}}$ represents the field fluctuation caused either by deformation in the uniformity of the field used, or else by regional changes in the $(\chi)$ of the system (Browne \& Semelka, 1999).

Both $\mathrm{T}_{1}$ and $\mathrm{T}_{2}^{*}$ relaxation times may be reduced by employing magnetic contrast agents, paramagnetic Gd ion complexes being the most frequently utilized. The interesting point in the context of the present thesis is that superparamagnetic nanoparticles designed with such aim exist already in the market (e.g. in Advanced Magnetics Inc. (Pankhurst et al., 2003)). The superparamagnetic nanoparticles employed normally reach the saturation magnetization for the usual magnetic field strengths in MRI scanners. They 
A
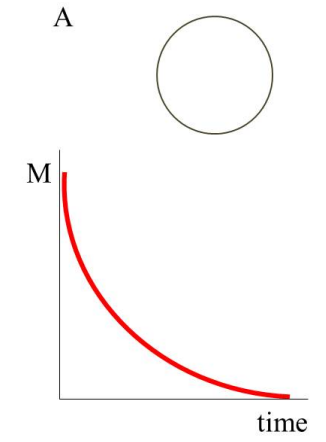

B

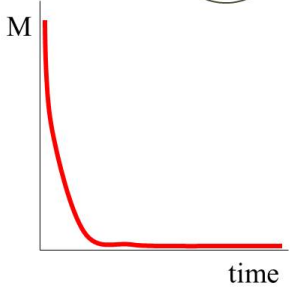

Figure 2.7: Influence of the incorporation of magnetic nanoparticles in the cells on $\mathbf{T}_{2}^{*}$ : A - unlabelled cells; B - the protons present in cells labelled by magnetic materials possess a smaller $\mathrm{T}_{2}^{*}$.

work by creating an important regional disturbing dipolar field which gives rise, by means of Eq. 2.5, to a marked reduction of $\mathrm{T}_{2}^{*}$ (Fig. 2.7) accompanying also a marked, but to a smaller degree, shortening of $\mathrm{T}_{1}$ (Elster \& Burdette, 2001).

The most common superparamagnetic material-based MRI contrast agents are SPIONs, generally with dextran or carboxydextran coatings. There are two formulas accepted nowadays for clinical treatment, ferumoxides and ferucarbotran, respectively, both used in MRI of liver. It has been proved that once administrated in the body through a vein, the SPIONs are eliminated via phagocytosis completed by the reticulo-endothelial system, and their capture is noticed in the healthy liver, spleen, bone marrow, and the lymph nodes (Wang et al., 2001).

Gene therapy. It is an innovative therapy (also known as magnetofection), based on magnetic nanoparticles use, for the treatment of genetic malfunctions, including cancers, neurodegenerative and cardiovascular diseases.

The main concept of this kind of therapy is to fight the lack of genetic order by generating outer proteins/peptides which raise the response of the immune system via insertion of exogenous DNA. In the case of in vivo, if magnetic particles are utilized, the starting part is the coupling of nucleic acids to positive magnetic nanoparticles. These particles are administrated intravenously and afterwards external magnets of high gradient are utilized for entrapping the particles when they flow inside the blood stream. 


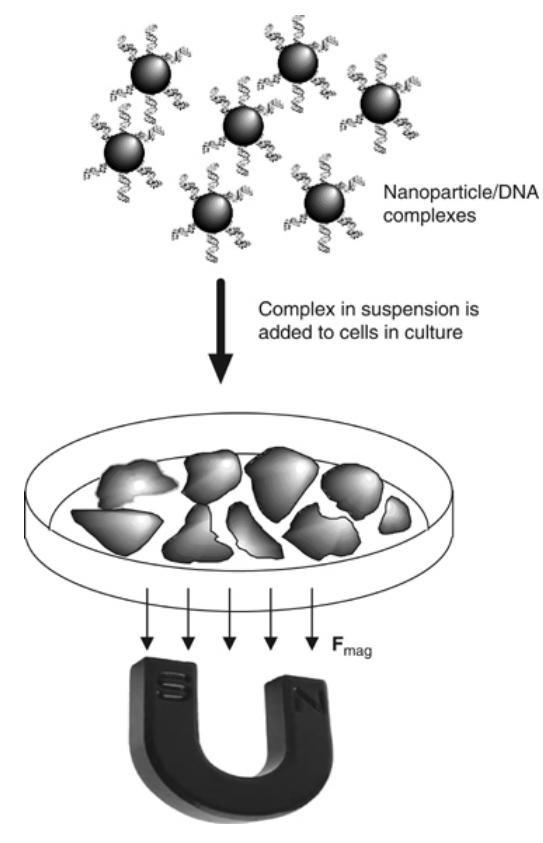

Figure 2.8: Schematic picture of the magnetofection technique under in vitro conditions. The carrier is linked to the nanocompounds, which are introduced to the cell culture. A permanent earth magnet (or electromagnet) is located below the culture dish and nanocompounds are drawn downwards. This causes the rise in the sedimentation rate and speeds up the transfection process. $\mathrm{F}_{\text {mag }}$ represents force pushing down the nanocompounds by the magnetic field (Dobson, 2006a). 


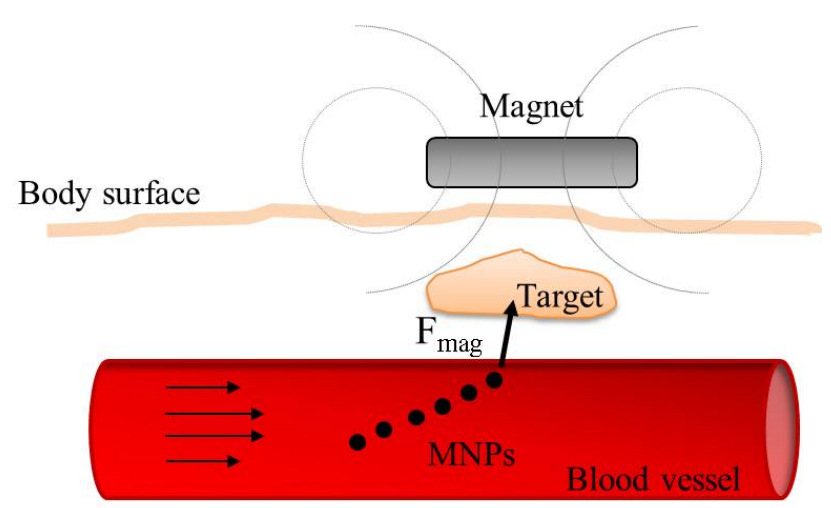

Tissue

Figure 2.9: A diagram representing the gene targeting process under in vivo conditions employing nanostructures, which may be also a model illustration of magnetic drug delivery system displayed in cross-section: a magnet situated outside the human body is pulling the nanoparticles loaded with drug or nucleic acid, which are floating in the circulatory system.

Once entrapped, the particles are maintained at the target site, where they are taken up by the tissue. The therapeutic genes can be liberated either by enzymatic cleavage of the cross-linking molecules, charge interactions or polymer matrix degradation (Banerjee et al., 2010). In the case of in vitro magnetic transfection employing nanostructures, a system formed by nanocompunds and DNA is added to the cell culture, in which the magnetic field gradient generated by means of a permanent magnet (or electromagnet) located beneath the cell culture rises the sedimentation rate and subsequently the velocity of the transfection process (Fig. 2.8, 2.9).

\subsubsection{Physicochemical factors determining the fate of nanopart- icles in the body}

\subsubsection{Geometry}

It can be stated that particle size and shape are among the most significant properties controlling the stability (and, in general, the physiological fate) of nanocarriers in both in vivo and in vitro experiments. The size highly affects the magnetic moment of the nanoparticles and the way in which they respond to the applied magnetic field. It has been demonstrated that 
the smaller the particle diameter, the lower the saturation magnetization (Di Marco et al., 2006). What is worthy to note is that the particle diameter has also been reported to govern the signal in magnetic resonance imaging of iron oxide nanoparticles (e.g. transverse relaxivity, $R_{2}$, values), that may have important implications on the interpretation following a diagnostic exam (Wiessleder et al., 1990). Other features in which particle diameter affects the in vivo nanoparticle role are extravasation, targeting, circulation times, internalization, immunogenicity, degradation, flow properties, clearance and uptake mechanisms (Fig. 2.10)(Kohane, 2007).

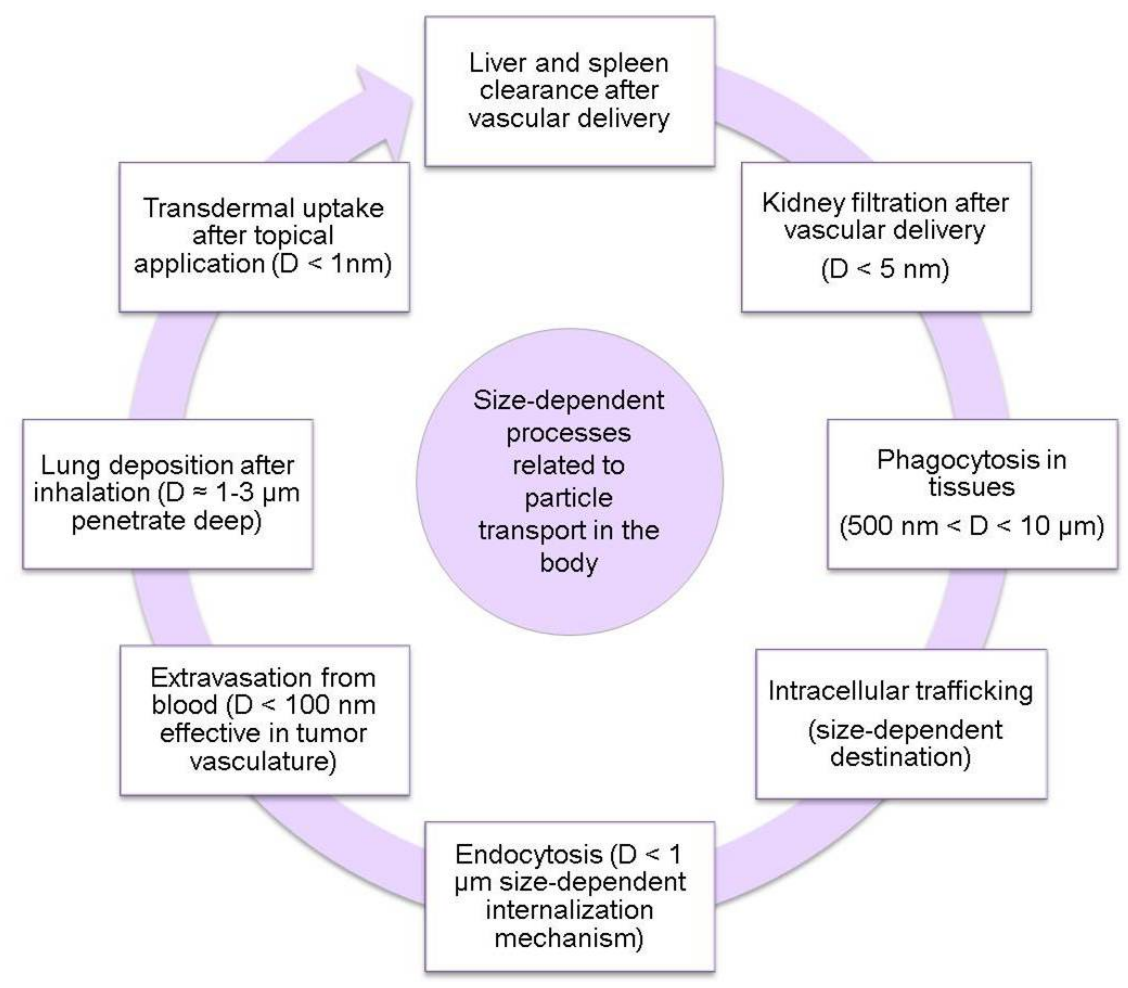

Figure 2.10: Schematics of particle transport processes occurring in the human body which are determined by particle size. Nanocomposites may go through biological boundaries by many different mechanisms. The majority of them influence biodistribution and clearance processes of particles in the body and are characterized by particle diameter (Mitragotri \& Lahann, 2009).

Size also governs particle motion and adhesion processes in the cir- 
culatory system, airways or digestion organs. There are different mechanisms responsible for clearance in blood. Due to the fact that the smallest sizes of capillaries within the human body are around $4 \mu \mathrm{m}$ (Schmidt \& Thews, 1995), big particles tend to be entrapped and held back in the lungs (Kreuter, 1985). Thus, bigger magnetic nanoparticles or their agglomerates may be captured, bringing about emboli inside the circulatory system of the lungs (Kreuter, 1983). As mentioned before, the majority of nanocompounds administered intravenously are identified as 'strange'objects and they are finally removed at once by the macrophages. In the case of particles with sizes smaller than the minimum capillary diameter, they are captured by the RES system, up to $60-90 \%$ in the liver and the rest in the spleen (Müller et al., 1997). At the same time there is a great probability that nanoparticles not exceeding $100 \mathrm{~nm}$ in size will be engulfed by liver cells, because holes in the endothelium of liver sinusoids (the venous cavities through which blood passes in various organs) range from 100 to $150 \mathrm{~nm}$. At the same time, nanoparticles having particle diameters greater than $200 \mathrm{~nm}$ tend to be taken up through sinusoid vessels of the spleen (Moghimi et al., 2001). Using particle diameter the process of uptake may be categorized more specifically into phagocytosis (which occurs for particles of all sizes) and pinocytosis (only in case of nanoparticles smaller than $150 \mathrm{~nm}$ ) (Massen et al., 1993; Müller et al., 1997).

When nanoparticles of sizes greater than $10 \mathrm{~nm}$ are found within the body they cannot pass through the endothelium (Schütt et al., 1997). Nevertheless, this penetrability barricade can be raised when any pathology in the body occurs, that is tumor permeation or protective reaction to irritation, injury, or infection. At that place, the permeability frontier may be raised to permit even particles of $700 \mathrm{~nm}$ size (Moghimi et al., 2001).

Factors like size and surface chemistry are as well considered to influence opsonization process. In one of the studies where the effect of different particle sizes $(14,50$ and $74 \mathrm{~nm})$ on their uptake within the body was studied, it was determined that the uptake was importantly greater in case of nanoparticles of $50 \mathrm{~nm}$ in size. Similarly, in another study it was found that the process of phagocytosis for polystyrene nanoparticles is as well strongly determined by particle diameter, with its maximum happening in a size limit from 2 to $3 \mu \mathrm{m}$. Interestingly, this particle diameter range is analogous to the bacteria size range (See Fig. 2.11) and they can be believed the most widely known objectives of macrophages (Mitragotri \& Lahann, 2009). 

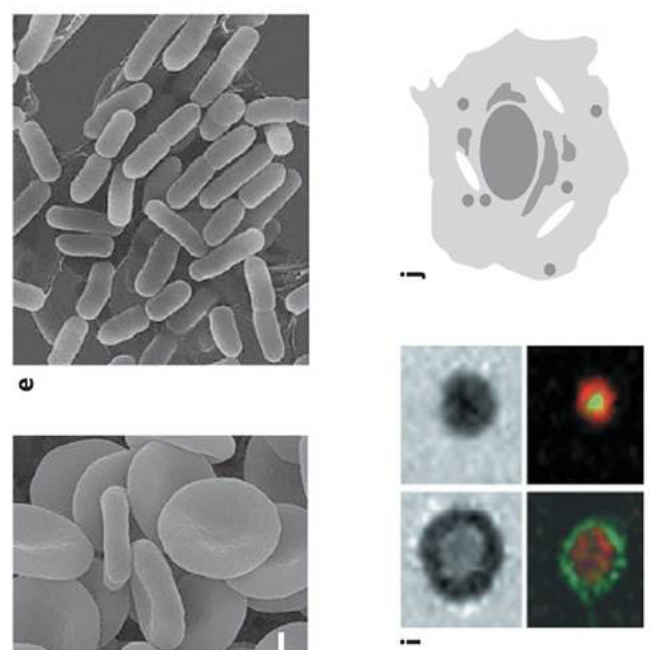

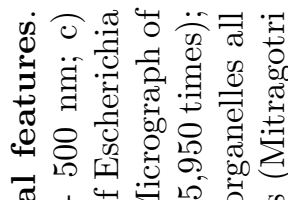

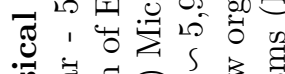

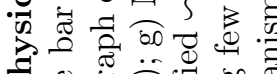

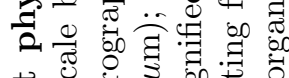

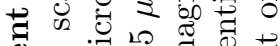
Ð

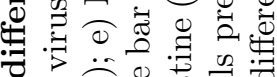

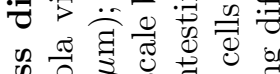

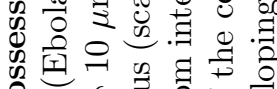

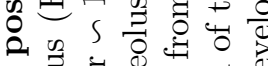

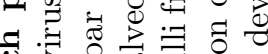
50 $\overline{5}$

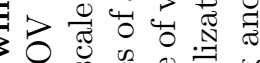
o 000 .

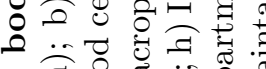

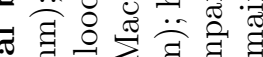
응

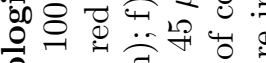

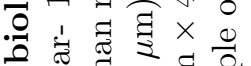

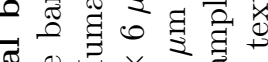

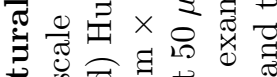

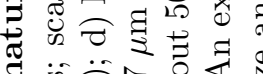

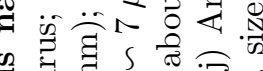
号. สี क्षे

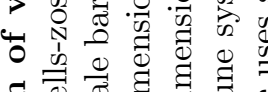

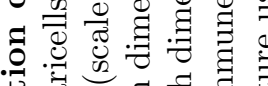

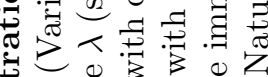

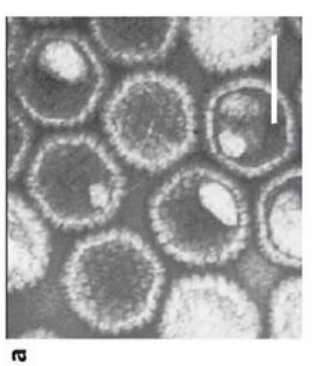
药

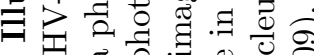

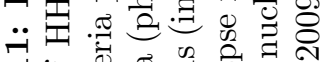
ن

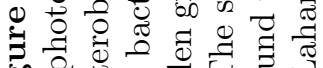

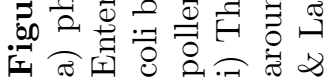


Shape is another particle feature (Fig. 2.12) greatly influencing biomedical applications as well. There exist theoretical studies which show the advantages of non-spherical nanoparticles in some biomedical applications like drug delivery which work on the basis of the particle shape impact on cellular internalization and vascular dynamics. For instance, the process of phagocytosis by macrophages is to a large extent controlled by particle shape. Actually, it is the restricted geometry of nanocompounds in the moment of attaching to the cell, not the particle size as a whole, that may control if macrophages start internalization process. For instance, a macrophage finding a particle of elliptical disc shape ended with a sharp point can incorporate in some minutes, while this process takes up to $12 \mathrm{~h}$ with a flat particle. In summary, the local particle shape is decisive for the beginning of phagocytosis, determined by creation of an actin ring below the macrophage layer. In another study it has been found that at a lesser scale the process of internalization of the nanoparticles of cylindrical shape is determined by their aspect ratio. When the particle aspect ratio happens to be three, the internalization occurs about four times faster than for its spherical analogue having identical volume (Gratton et al., 2008; Muro et al., 2008). 

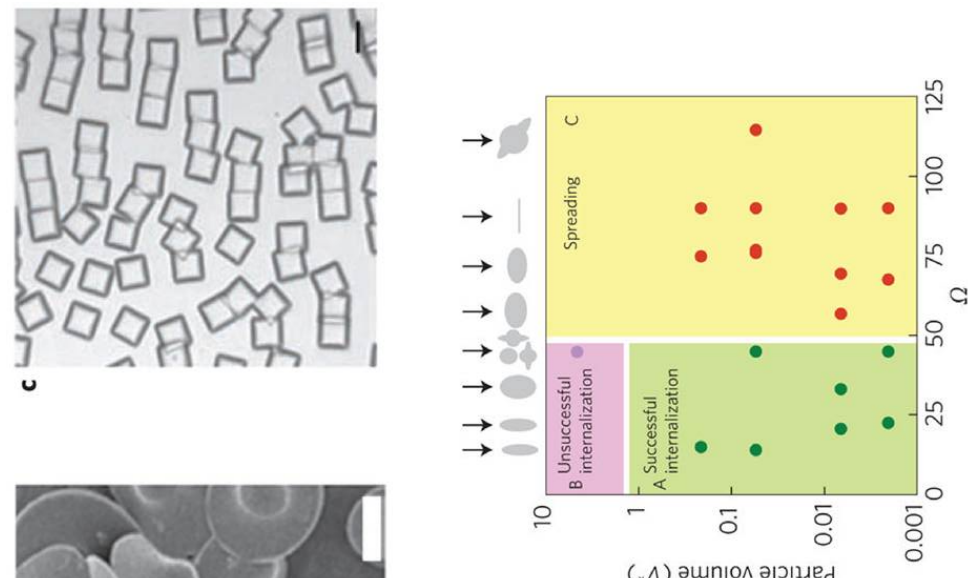

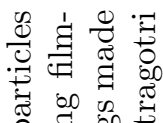

苋.

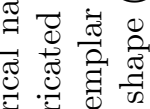

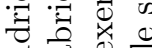

需

它氙芯

㟧弄员?

究

द्व్

讯 灵

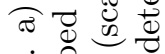

घ)

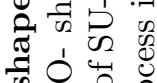

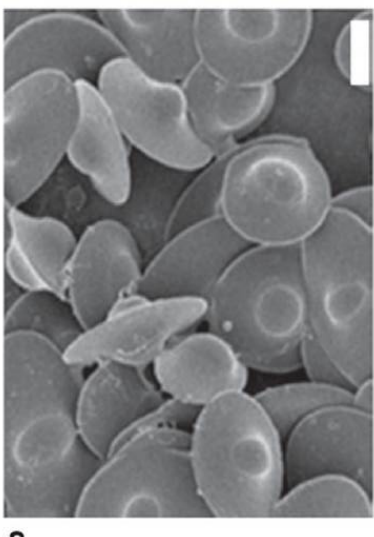

$(* \Lambda)$ әширо әрэ!นе

का

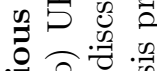

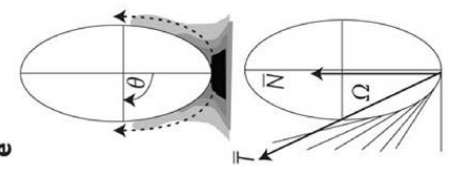

สี

द

考的要

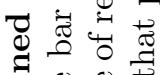

ส

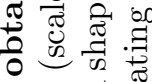

of

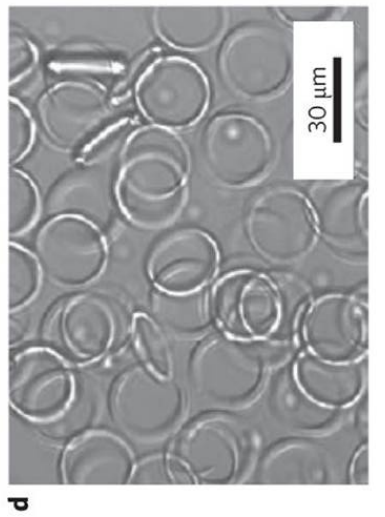

过司运

西茟

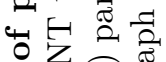

a 0 品

气

要岸

the

$\Xi \stackrel{\Xi}{\Xi} \Xi$

$\exists$ 目

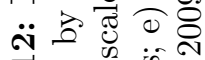

ᄀ่

. $\cong$ घ

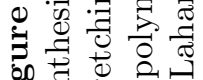

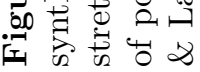


Many research studies have already demonstrated that the use of compounds with controlled shapes may determine advantages inaccessible with spherical particles. Spherical nanocompounds have to be smaller than 200 $\mathrm{nm}$ in order to travel through the spleen whereas disc-shaped particles as red blood cells (which are about $10 \mu \mathrm{m}$ in size), do that passage routinely. There exist theoretical studies which have already demonstrated many advantages of non-spherical compounds usage for biomedical applications such as drug delivery (Mitragotri \& Lahann, 2009). For instance, they expect that nanocompounds of oblate shape may adhere with higher efficiency to the endothelium cells forming vessels than particles of spherical shape of identical quantity would achieve (Decuzzi \& Ferrari, 2006), a property which is crucial for targeted drug delivery to endothelium.

\subsubsection{Surface charge}

After intravenous injection, magnetic colloids meet with a somewhat basic $\mathrm{pH}$ (physiological pH 7.4), and comparatively high ionic strength (around $140 \mathrm{meq} / \mathrm{L}$ ). These physiological conditions combined with magnetic forces provoke the situation in which the particles often tend to aggregate, mainly due to van der Waals and/or magnetic dipole-dipole attractive interactions. Hence, particles stability under in vivo and in vitro conditions can be only achieved when the following effects are balanced: i) particles interact with each other thanks to their hydrophilic nature; ii) suitably charged surfaces repel each other; iii) when there is a suitable shell surrounding nanoparticles which provides steric stability (Duran et al., 2008).

It can be said that the charge of magnetic colloids extremely matters throughout the process of endocytosis. Generally, the nanocompound uptake occurs at a slower rate in the case of particles with negative charge because of repulsive effects of the negative cell membranes. Additionally, in vitro studies have proved that endocytosis indicators are minimum when the electrokinetic potential value is in the vicinity of zero (Kellaway, 1991). On the contrary, phagocytosis grows when the surface charge is finite, no matter if it is negative or positive (Müller et al., 1997). The blood circulation time is shorter if the surface charge of particles is high (Chouly et al., 1996).

Some new studies have shown that particles of polystyrene functionalized with amine went through more phagocytosis processes when contrasted to particles possessing sulfate, hydroxyl and carboxyl groups attached to their surface. Due to this fact and as mentioned earlier, it is widely recognized that particles with positive charge experience faster cell uptake when 
compared to neutral and negatively-charged ones. It is also known that for positive nanoparticles the non-specific internalization process occurs faster and their half-life in the circulatory system is significantly brief (Alexis et al., 2008).

It has recently been shown that thiolated gel nanoparticles with size of $\sim 250 \mathrm{~nm}$ and with $\zeta$ values of $-5 \mathrm{mV}$ get accumulated in the tumor area in larger amounts then the nontiolated ones. In addition, the half-life of thiolated particles, rated at about 3 hours, suggests that they can happen to be collected within the tumor area. Moreover, the thiolated nanocompounds were discovered to be taken up faster by the spleen.

\subsubsection{Surface thermodynamics}

The tendency of particles to aggregate in aqueous solution is not only determined by the surface charge but also by their wettability, which determines if particle-water interfaces are thermodynamically preferred (posses a lower surface free energy) than particle-particle ones. It is a fact that the hydrophilic/hydrophobic characteristics of the magnetic colloids define how they will later interact with the plasma proteins under physiological conditions (known also as opsonization process). Generally, opsonization takes place faster in case of nanoparticles with hydrophobic nature (Neuberger et al., 2005). As a result, this agglomeration can impede the efficiency of iron oxide nanoparticles in drug delivery (smaller amount of drug loading) because of the smaller surface area associated to larger particle diameters.

These phenomena may be well estimated without difficulties by means of the van Oss theory (van Oss, 2006). Employing this model, all the surface free energy ingredients of the nanostructures can be calculated (van Oss et al., 1988; Chibowski \& Gonzalez-Caballero, 1993; Chibowski, 2003, 2007), and this can be applied for the estimation of their thermodynamic stability in aqueous solutions. Generally, these ingredients may be obtained for example in contact angle determinations (Chibowski et al., 2011; Duran et al., 2008). As a matter of fact, contact angle measurements do also make their contribution regarding the estimation of differences in the interaction free energy shown by magnetic iron oxide coated with hydrophilic coating (Duran et al., 2008).

\subsubsection{Pharmacokinetics, biodistribution and biological fate}

In targeted drug delivery, magnetic nanoparticles administered into the body by intravenous injection go through three consecutive stages: i) they 
are led to the target using an external magnetic field; ii) they remain within the target area until whole whole drug loading is liberated; iii) finally, they are expelled from the human body. The magnetic nanoparticles transportation through the circulatory system is supported by the hydrodynamic forces of the blood flow. In a short time following intravenous injection, an instantaneous interaction between magnetic nanoparticles and the opsonins (plasma proteins) takes place. This gives rise to the opsonins adsorption onto the surface of nanoparticles, also known as opsonisation. This type of adsorption is very important for the biological destiny of the magnetic colloids (e.g. biodistribution and clearance process) (Blunk et al., 1993).

The opsonised magnetic colloids are eliminated rapidly from the circulatory system because of their entrapment by the macrophages of the liver (Küpffer cells), of the bone marrow and of the spleen, that compose the reticuloendothelial system (RES) (Bochard, 1993). This type of collecting of opsonised magnetic colloids in the RES parts of the body is believed to be positive/advantageous if these parts of the body are the target area. Therefore, in order to transfer magnetic colloids to target organs not belonging to the RES systems it is necessary to diminish the fast opsonisation and elimination described. As already mentioned, this may be obtained by coating onto the magnetic non-fouling shells leading to a yield shielding effect (known as well as 'stealth property') (Arruebo et al., 2007). Until now, polyethylene glycol (PEG) is the most suitable and the most popularly employed polymer with hydrophilic characteristics to impede administrated magnetic colloids from being expelled from the body by the RES system. Two principal characteristics of PEG, that is its chain length and its packing density, slow the process of opsonisation as a result of steric hindrance effect and by means of that extend the half-life of magnetic colloids within the circulatory system (Gref et al., 1994). In case of PEG possessing a chain with molecular weight from $2 \mathrm{kDa}$ is considered to be the most appropriate for this goal, as shown using different nanoparticle systems created for the drug delivery purpose (Matsumura, 2008).

When dealing with tumors, the magnetic colloids with long half-life get to the target site by means of passive extravasation, profiting from the augmented tumor permeability proceeding from the EPR effect in the tumor vasculature (Maeda, 2010). Otherwise, magnetic colloids can be lively gathered within the tumor site using an external magnetic field. So as to prevent magnetic nanoparticles from being eliminated from the targeted site within a short time, an external magnetic field must be strong enough to overcome the hydrodynamic blood flow (e.g. for arteries $>10 \mathrm{~cm} / \mathrm{s}$ and 
for capillaries in a range between $0.05-0.1 \mathrm{~cm} / \mathrm{s}$ ). The capacity of having magnetic colloids trapped is also conditioned by the particle diameter ( $\mathrm{Du}-$ ran et al., 2008). In a practical way, the magnetic field strength should be $\geq 1 \mathrm{~T}$ to obtain effective entrapment of magnetic colloids despite the brownian and hydrodynamic forces existing in the blood vessels (Senyei et al., 1978). Generally, small nanoparticles in usual blood vessels are trapped only at distances smaller than $0.5 \mathrm{~cm}$ from the skin, in the presence of a magnetic field applied externally. However, in the tumor vessels, magnetic nanoparticles can be entrapped up to $1.5 \mathrm{~cm}$ due to the porous vasculature. The entrapment distances increase observably when the particle size raises, extending up to $6 \mathrm{~cm}$ in case of healthy blood vessels and even up to $10 \mathrm{~cm}$ for the tumor ones (Duran et al., 2008).

Influence of the administration route. If nanoparticles are administrated locally by injection just beneath the skin or within the tumor, they will undergo gradual penetration into the interstitial parts surrounding the area of injection and they are progressively taken up by the lymph vessels. Because of this, locally administrated nanocompounds with sizes smaller than $60 \mathrm{~nm}$ are employed for targeting lymph node therapy and imaging (Reddy et al., 2012).

Another example is a study of fluorescent magnetic colloids coated with PVP (polyvinyl-pyrrolidone) and with particle size of about $50 \mathrm{~nm}$. These nanocompounds were administrated by means of inhalation to mice during four weeks and they were significantly collected in the liver and afterwards in male gonad, in the spleen, lung and even in the brain. Trace amounts of administrated magnetic colloids were found in the nasal cavity and also in heart and kidney. Therefore, the nanocompounds thus administrated were able to pass through the narrow windows like the epithelium within the lungs or even the endothelium inside the brain and testis blood vessels (Kwon et al., 2008). Another example of passage through the blood brain barrier was reported by (Kim et al., 2006) using magnetic nanoparticles with outer silica shell and stabilized with PVP, administrated to the interior of the peritoneum by injection.

There exists the misconception that the nanoparticle distribution within the body after intravenous administration is identical as that when nanocompounds are introduced in the circulatory system from other tracts like respiratory system, skin or gastrointestinal tract. Fig. 2.13 presents basic dissimilarities in nanocompound relocation paths to circulatory system and body organs depending on whether nanocomposites are administrated intravenously or through the respiratory system. 


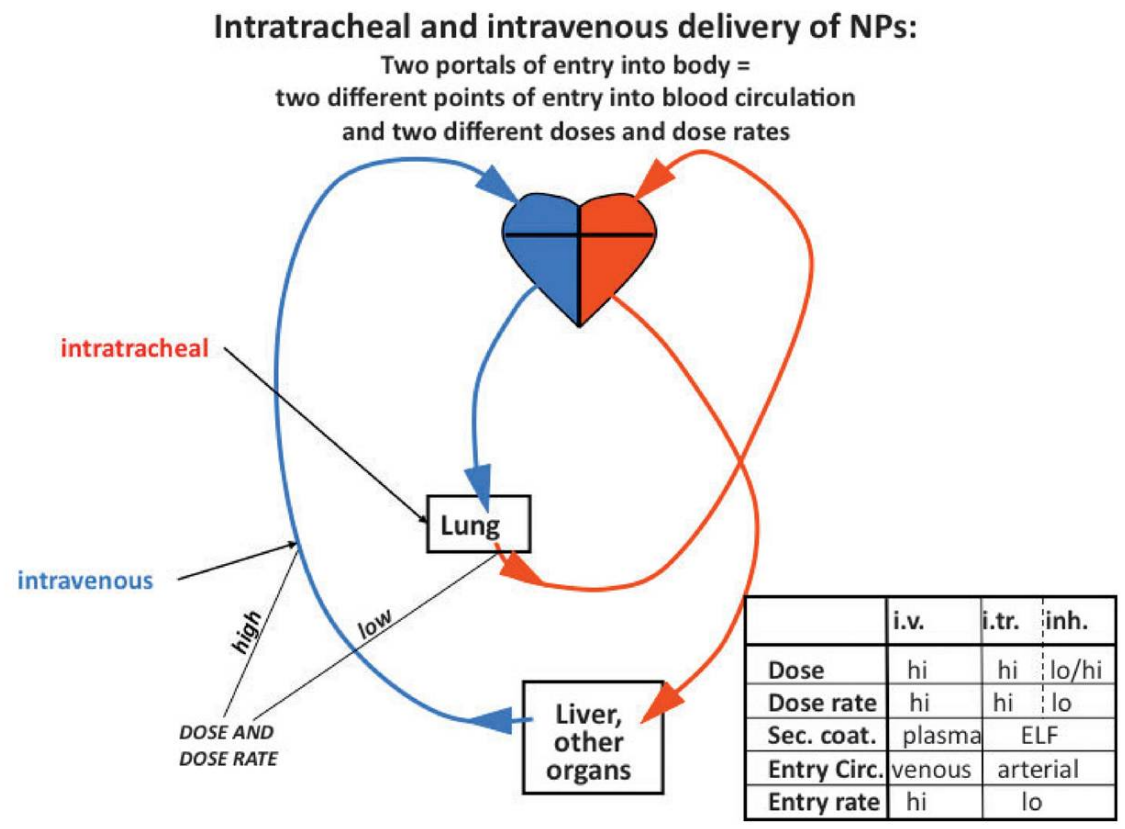

Figure 2.13: Schematic illustration of the pharmacokinetics of nanocomposites within the human body depending on the port of entry. Identical nanocompounds which were delivered to the lung (by inhalation or intratracheal instillation) or administered by intravenous injection, interact with various biological objects and will get various secondary layers (Oberdörster, 2009). 
Elements affecting the tissue/cell penetration. Regarding the NPs dispersion within tissues, particle size exceeding $10 \mathrm{~nm}$ normally limits their spreading out through little pores in vessels within regular endothelium (Schütt et al., 1997). The situation is quite the reverse when the border of the endothelium is impermanently endangered because of not genuine means such as heating or radiation, or either as an outcome of particular pathological situation like tumor permeation, and then nanocompounds with particle diameter smaller than $700 \mathrm{~nm}$ may pass into the endothelium without limits (Moghimi et al., 2001). It is worth to mention that degradation of iron oxide colloids depends on their surface approachability to water and this also clearly depends on the type of particle coating. Magnetic colloids with comparable coatings, although having various particle diameters are degraded within liver with much the same velocity. For example, magnetic nanoparticles of $58 \mathrm{~nm}$ and $12 \mathrm{~nm}$ possessing the same carboxydextran layer were administrated by intravenous injection ( $5 \mathrm{mg} \mathrm{Fe} / \mathrm{kg}$ ) and they exhibited comparable velocities of hepatic degradation. Similar conclusions have been reached regarding hepatic metabolization process of the above mentioned Ferumoxide and Ferumoxtran-10 commercial materials (Jung, 1995; Varallyay et al., 2002). It must be underlined that even though the process of hepatic nanoparticle clearance is not controlled by dissimilarities in the particle diameter, the amount of nanocompound uptake can, by contrast, affect the velocity of clearance (Reddy et al., 2012).

As for the cellular level, the process of nanoparticle passing into is highly affected by their particle diameter (Hillaireau \& Couvreur, 2009). After entering the cell, the nanoparticles of iron oxides are enclosed in the lysosome, and then the metabolism into iron and oxygen is assumed to happen under conditions of low enough $\mathrm{pH}$ and also in the presence of various hydrolytic enzymes taking part in the iron degradation process. The most important benefit of using nanoparticles of iron oxides relies on the fact that iron ions coming from their metabolism, are later employed again by the cells by way of usual biochemical routes of the degradation (Schulze et al., 1995; Singh et al., 2010).

\subsubsection{Toxicity and biocompatibility}

\subsubsection{General concepts}

In order to employ the capacity of nanotechnology in medicine, there is a need of much attention to safety and toxicity of the nanoparticles injected in the body. The number of experiments on magnetic particles tox- 
icity is scanty, however nowadays we observe a tendency to investigate this issue more carefully and with more frequency. Complete estimation of wanted versus adverse effects is needed for safety employing nanomaterials in various biomedical applications. The first toxicity study with magnetic materials was carried out on carbohydrate-coated magnetic nanoparticles in nude mice and the results demonstrated no median lethal dose $\left(\mathrm{LD}_{50}\right)$ nor modifications in the blood environment and biochemical informations were examined after the administration of magnetic particles. On the other hand, if the volume of magnetic particle suspension exceeded $10-20 \%$ of the blood volume, some cases of lethargy and repulsive behaviour to food were noticed (Lübbe et al., 1996).

A constantly developing production and usage of nanomaterials, and especially spherical and fibre-like nanoparticles, for different biomedical applications and in consumer goods, has attracted much attention their safety for human health. It has been shown in recent years that some nanocomposites provoke more serious toxic effects than larger objects with identical chemical structure (Oberdörster et al., 2007), and it is quite recently that the need to estimate the safety of nanomaterials for future scientific development must be taken seriously (Oberdörster, 2009). 


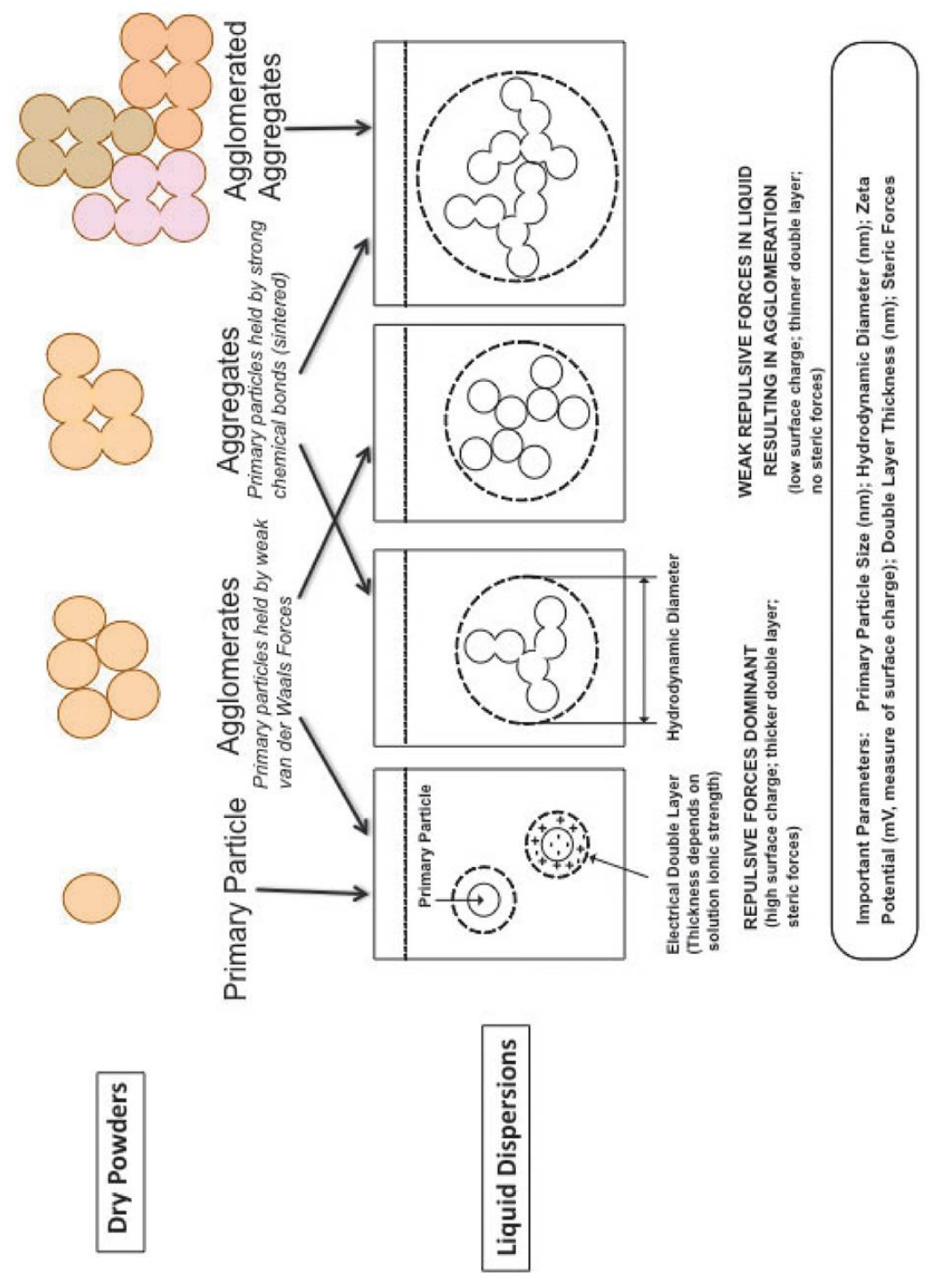

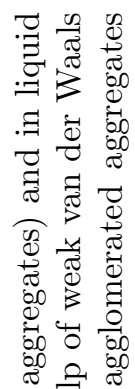

픙

के

象

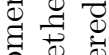



苍苍

豙

氙

范

承

.

के

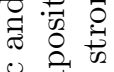

当 영

敢

궁

氖

范苛总

㟧

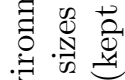

द्व

.

$\exists$ :

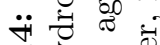

서 $\vec{G}$

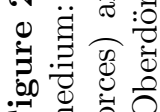


In all the examples mentioned up to this point in this Thesis, living cells are at some moment set in contact with different nanoparticles, mostly inorganic, coated or not. However, systematic studies on the possible adverse effects of the introduction of the NPs in the body are absent (Soenen et al., 2011), and in fact there are many aspects of the problem that should be considered, ranging from the kind of particles and coatings used to the type of cells employed and the assay chosen for toxicity evaluation. Some reviews have been recently elaborated on the subject (De Jong \& Borm, 2008; Hussain et al., 2009; Lewinski et al., 2008; Maurer-Jones et al., 2009; Pelley et al., 2009; Singh et al., 2010; Soenen et al., 2011), and so we will give only a brief account, mainly focused on the kind of particles used in our investigation. We will hence not consider the particular case of drug vehicles designed to be delivered to the patient though respiratory ways (Oberdörster, 2009), as in this route the first unwanted effects can occur already in the lung cells, and only later, after passing the alveolo-capillary barrier, they can get into the bloodstream and spread in the body, where either beneficial or undesired effects can occur as well.

Dose concept. In the case of nanoparticles, the relation between their dose and the organism reaction (to them) needs exceptional caution, partly because the traditional and widely utilized standards of dose measurement can be incomplete, and often focussed on mass. For instance, the US National Ambient Air Quality Standard for the period of time of $24 \mathrm{~h}$ in the case of fine nanoparticles $\left(\mathrm{PM}_{2.5}=\right.$ airbone particles $\left.<2.5 \mu \mathrm{m}\right)$ is 35 $\mu \mathrm{g} / \mathrm{m}^{-3}$. Although this may seem to be a comparatively small concentration, it may signify an enormous concentration for ambient nanoparticles (about $20 \mathrm{~nm}$ in size) with number concentrations as high as $1 \times 10^{6}$ particles $\mathrm{cm}^{-3}$. Therefore, although all the above mentioned standards are expressed by mass, it may not be significant in case of nanoparticles. In various toxicological experiments it was demonstrated that the most suitable dose-metric for contrasting the influences of various particle diameters and various types of nanocompounds is the nanoparticle surface area and not their number or mass (Oberdörster et al., 2005).

The list below presents some physico-chemical features of nanocompounds which have effect on their biological and/or toxicological activeness. As mentioned earlier, there are nanoparticles features of relevance for toxicology are (See also Fig. 2.14):

i) size (airbone, hydrodynamic);

ii) size distribution;

iii) shape; 
iv) agglomeration/aggregation;

v) surface properties, such as:

$$
\begin{aligned}
& \text { - area (porosity) } \\
& \text { - charge } \\
& \text { - reactivity } \\
& \text { - chemistry (coatings, contaminants) } \\
& \text { - defects }
\end{aligned}
$$

vi) solubility (lipid, aqueous, in vitro);

vii) crystallinity.

To characterize better one of the feature presented above, it can be mentioned that the particle diameter may affect cells in vitro in various ways. For example, nanocompounds of $30 \mathrm{~nm}$ cause higher cytotoxicity than $500 \mathrm{~nm}$-sized nanocomposites. When these nanoparticles are left in contact with the A549 epithelial cell line, different effects of size and dose on the cell injury are observed(Reddy et al., 2012). Smaller magnetite nanoparticles (30 nm in size) provoked greater oxidative DNA harm effect than $500 \mathrm{~nm}$ particles (for concentrations of $80 \mu \mathrm{g} / \mathrm{mL}$ ), while in contrast, for lower concentration in the order of $40 \mu \mathrm{g} / \mathrm{mL}$, no cytotoxicity was observed for either size.

Toxic effects provoked in vitro can be connected with certain cell culture alterations caused by nanocomposites incubation. For example, in many cases bare magnetic colloids possess negative surface charge in aqueous solutions because of the attachment of $\mathrm{OH}^{-}$ions to their surface. This can encourage protein adsorption, and, in addition, chloride ions may as well be inclined to compete in order to be attached to iron ions, thus changing the $\mathrm{pH}$ of the surrounding environment at the same time while they do so with the surface properties of the nanocompounds. These kinds of interactions with the culture medium cause changes in the ionic concentration and protein role, and they may provoke cell separation and result in cell death (Mahmoudi et al., 2010).

Cytotoxicity can be more significant and noticeable under in vivo than in vitro conditions due to the fact that in the culture medium the nanocomposites and their metabolism products are permanently in close vicinity to the cells, whereas the situation in vivo conditions is different, since the nanocompunds are ceaselessly removed from the body undergoing biodegradation processes. Therefore, it is clear that the estimation of in vivo safety of magnetic nanocompounds is fundamental for biomedical applications.

To mention few, various studies on the biocompatibility of SPION coating have been performed, for example, with or without PEG coating using 
in vitro grown human fibroblasts (hTERT-Bj1). The particles without coating brought about an important reduction (of about $64 \%$ ) in cell adhesion in comparison with that of the control cells, while in contrast nanocompounds with PEG coating did not show noteworthy alteration (Reddy et al., 2012). Maintaining nanocomposites with dextran coating in contact with human fibroblasts (TERT-Bj1) resulted in cytotoxicity and programmed cell death similar to that of nanocomposites without dextran coating. On the contrary, nanoparticles with albumin coating did not affect cell viability differently from nanocomposites with dextran coating and the bare ones (Berry et al., 2004). The nanocomposites with albumin layer brought about only insignificant cell membrane break, likely due to the fact that albumin interacts with phospholipids/fatty acids of the cell membrane. In general, it can be said that the characteristics of the coating material can importantly affect the cytotoxicity of magnetic colloids: identical coating onto distinct cores can also cause various toxicological consequences because of the participation of other factors (like size) (Reddy et al., 2012). It has been demonstrated that DMSA-coated magnetic colloids characterized by negative surface charge undergo strong endocytosis by different types of cells, and provoke a reduction in the viability of clonal cancer cell line (PC12). In the $48 \mathrm{~h}$ following exposure of these nanocomposites to nerve growth factor (NGF), the PC12 cancer cells usually become different within neuronal cells and start to origin mature neuritis that may be stretched to the outer border. In addition, a reduction in expression of protein connected with the axon function to send impulses away from the cell and other neuronal roles was determined at concentrations used in this study, indicating that toxicity of these negative nanocomposites depends on the dose (Reddy et al., 2012). Other studies have demonstrated that the coating material, even if yielding comparable surface charge to the final composites, may significantly change their cytotoxicity as for the dose (Reddy et al., 2012).

Effects on blood and the cardiovascular system. Cationic nanocompounds (polystyrene included) have been demonstrated to provoke hemolysis and blood coagulation, whereas normally anionic nanocomposites are rather non-toxic. The comprehension of this concept may be employed to impede possible effects of unintentional nanocomposite exposure (Gupta \& Gupta, 2005). From a different point of view, attempts have been reported ascertaining the possible increased risk of patients with cardiovascular problems if they are exposed to PM (particulate matter; particle mass fraction in ambient air) (De Jong \& Borm, 2008). Some toxicological investigations have shown that combustion and example nanomaterials may find their way to 
the circulatory system after inhalation or instillation and may intensify experimental thrombosis although it is not obvious if this was an impact of inflammation in the lung or nanomaterials moved to the circulatory system (Mills et al., 2005).

Uptake and impact of nanomaterials in the brain. Nanocomposites may enter the brain thanks to two mechanisms, the first of them is by carrying them over synapsis after inhalation through the olfactory system, and the second one is by taking them up through the blood brain barrier (BBB). As for the first way, it has been investigated firstly with exemplar nanomaterials like carbon, $\mathrm{Au}$ or $\mathrm{MnO}_{2}$ in studied inhalation patterns in rats (Oberdörster et al., 2005). Concerning the second manner, a number of studies have shown that neutral nanocomposites and anionic ones but at very low concentrations can be considered non-toxic for the BBB, while anionic nanomaterials at high concentrations and cationic ones were found to cause no effect on the BBB. Moreover, nanocomposites have been demonstrated to induce the formation of reactive oxygen species, as well as oxidative stress and this has been proved in the brain following inhalation of $\mathrm{MnO}_{2}$ nanocomposites. In addition, oxidative stress has been shown to be connected with the pathogenesis of Parkinson's and Alzheimer's diseases (De Jong \& Borm, 2008).

\subsubsection{Toxicity evaluation and existing data}

Superparamagnetic iron oxide nanoparticles. SPIO nanoparticles participate in the possible harmful effects of nanometer-scale materials: their large surface area (reactive nature), their tendency to go through membranes and tissue barriers (resulting in cell stress), etc. may possibly produce cytotoxicity which can be revealed by weakening the functions of the most important parts of the cell - mitochondria, DNA and nucleus (Singh et al., 2010).

The amount of researches in vivo carried out on humans is still low, but nevertheless one of them demonstrated that Ferumoxtran-10, which is a product consisting of superparamagnetic iron oxide particles smaller than $50 \mathrm{~nm}$ with dextran coating, produced side effects like diarrhoea, nausea or urticaria (although all of them were moderate and lasted a brief time) (Thoeny et al., 2009). Thus, it is clear that this matter is of great importance due to the fact that iron oxide nanoparticles can be metabolised and expelled from the circulatory system through iron metabolic pathways within an organism.

In the specific case of injected SPIONs, it must be recalled that iron is 


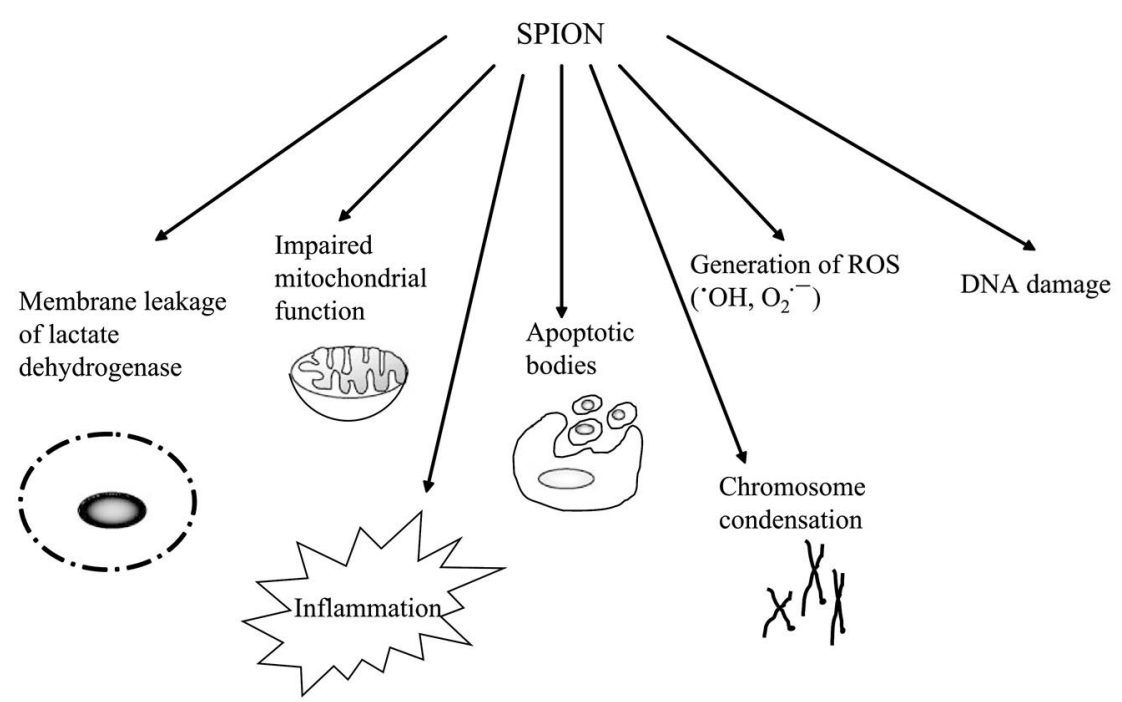

Figure 2.15: Types of cytotoxicity generated by superparamagnetic iron oxides nanoparticles (Singh et al., 2010).

an important element playing fundamental roles in cell signalling pathways. $\mathrm{Fe}^{3+}$ is normally transported by means of transferrin, which is capable of binding the cell membrane through specific receptors. In turn, ferritin stores the iron cell pool in the cytoplasm. Because of the significance of iron from the physiological point of view, SPIONs have long been considered as non-cytotoxic. However, although iron coming from the nanoparticle decomposition can go to the iron pool of the cell, it is precisely the small size of the SPIONs that can make them harmful, considering that they can reach moderately high concentrations in the cell. The effects are obviously dose-dependent, and, according to the revision carried out by (Singh et al., 2010), cytotoxicity can be expected, no matter the surface treatment of the particles for particle concentrations in the vicinity of $100 \mu \mathrm{g} / \mathrm{mL}$ or above.

The mechanisms by which the iron oxide nanoparticles can be toxic are very varied, as illustrated in Fig. 2.15. One of the most common is the generation of reactive oxygen species (ROS) when the cells are exposed to the SPIONs (Singh et al., 2010; Soenen et al., 2011).

Although in the case of iron oxide nanoparticles the dose injected intravenously ranges between $1.25 \%$ and $5 \%$ of the whole organism iron stock, there is a need to direct these nanomaterials with the help of magnetic 
field to a desired organ so as to derive maximum advantage for biomedical application, resulting in collecting a great number of nanomaterials within a targeted site. As a consequence, an excess of free iron ions can accumulate in the target site and result in a lack of balance in its homeostasis. This situation can give rise to abnormal cellular responses together with toxicity, DNA damage, inflammatory states or oxidative stress generation. Iron oxide nanoparticles can also produce some (geno)toxic effects by ROS generation. After being internalized, iron oxide particles probably undergo degradation to iron ions inside the lysosomes with the help of some hydrolysing enzymes active when $\mathrm{pH}$ is low. This free Fe may possibly go across the biological membranes, and further these free ferrous ions $\left(\mathrm{Fe}^{2+}\right)$ may react with $\mathrm{H}_{2} \mathrm{O}_{2}$ as well as with oxygen generated by mitochondria to generate hydroxyl radicals which tend to react very readily, and ferric ions $\left(\mathrm{Fe}^{3+}\right)$ through the Fenton reaction:

$$
\mathrm{Fe}^{2+}+\mathrm{H}_{2} \mathrm{O}_{2} \longrightarrow \mathrm{Fe}^{3+}+\mathrm{OH}^{+} \mathrm{OH}^{-}
$$

As a consequence, hydroxyl radicals produced by the free Fe may cause damage of DNA, polysaccharides, proteins and lipids under in vivo conditions.

It is clear that $\mathrm{pH}$ will be an essential parameter affecting the generation of these species and, in general, the chemical degradation of the NPs: the acidic character of endosomes and lysosomes favors the leaching of free iron into the cytoplasm, eventually leading to a complete loss of the magnetic response of the particles. This process can be controlled by proper coating again (Soenen et al., 2010).

In addition, the presence of the particles in the nucleus or its vicinity can produce alterations in the signaling pathways of the cell, including: a) changes in protein of gene expression provoked by particles in the perinuclear region; b) mRNA degradation induced by ions released by the particle's decomposition process; c) altered gene expression associated to the cell stress provoked by the particles, among others.

Coated superparamagnetic iron oxide nanoparticles. Concerning the second component of our particles, the silica coating, the many uses described for silica have also led to many investigations on possible harmful effects of $\mathrm{SiO}_{2}$ particles at the nanoscale (De Jong \& Borm, 2008). Silica nanocomposites exposure gave rise to raised ROS amounts and decreased glutathione quantities being evidence of the growth in oxidative stress. Another investigation demonstrated that at much smaller concentrations ( $\sim$ 
$0.1 \mathrm{mg} / \mathrm{ml}$ ) an important decrease in cell viability is noted. Moreover, it was found that cationic $\mathrm{SiO}_{2}$ nanocomposites functionalized with amino-hexylamino-propyltrimethysilane exhibited no or very low cytotoxicity (De Jong $\&$ Borm, 2008). As can be noticed, no conclusive results have been reported, though, since they have been proved to be both toxic (Chang et al., 2007) and non-toxic (Ravi Kumar et al., 2004).

Because of the fact that gold is the final coating layer of the nanoparticles here considered, it seems interesting to briefly consider whether toxic effects can also be associated with this material. Many authors agree about the safe use of gold nanoparticles in biomedical applications, including drug and gene delivery and imaging (Soenen et al., 2010). The exception is mentioned by (Soenen et al., 2010), regarding the very small size fraction of typically used gold particles (4-5 $\mathrm{nm}$ and below), because in that case they can go through the nuclear membrane and attach to DNA, producing potentially (geno)toxic effects. The harmful effects reported are generally ascribed to the stabilizing agent used: for instance, CTAB has been demonstrated to be very toxic, even in low concentrations, whereas particles with PEG coating, after clearing of CTAB, have not demonstrated cytotoxicity. Gold suspensions are as well utilized to fabricate nanoshells consisted from gold and copper, or gold and silver to operate as MRI contrast agents. Another ones made from gold and silica are employed in photothermal ablation of tumor cells. When gold nanocomposites are under in vitro conditions but they have not already reached the tumor cells, they did not exhibit any cytotoxic effect, while as attached to the tumor cells may provoke their death after laser activation. Favourable data were as well achieved after in vitro experiments with gold nanocompounds and using photothermal ablation (De Jong \& Borm, 2008).

\subsubsection{Minimizing toxicity}

The number of unanswered questions and needs in the evaluation of the toxicity of SPIONs is really large (De Jong \& Borm, 2008; Soenen et al., 2011):

i) A thorough characterization of the particles is required, concerning their size, stability, chemical inertness, interaction with plasma proteins, charge and wettability, ...

ii) The cytotoxic evaluation should follow well established, reproducible routines: cell type, assays for cell viability, ...

iii) Setting up the parameters defining toxicity are needed. 
iv) Is there a need of safe regulation in the use of these particles?

v) Are the nanoparticles (and not only their use in humans) potentially harmful themselves?

In spite of these uncertainties, some indications can be given as to the optimum conditions aiming at the minimization of toxicity. We follow (Soenen et al., 2011) for this:

i) The size and shape of the NPs are determinant for the toxicity, although there is a general agreement that the cell availability is most compromised if the diameter is below $5 \mathrm{~nm}$. It is also recommended to have a rather narrow size distribution, so that our efforts in producing monodisperse particles are really fruitful. Concerning shape, the majority of experiments are carried out with spherical particles, and where other geometries as nanorods have been tested, less toxicity has been ascribed to the latter. It is not clear, though, whether this is just a manifestation of slower diffusion of the non-spherical particles through the cell membrane, and hence hindered internalization.

ii) As has been mentioned before in this Thesis, surface charge is determinant, not only because of its effect on suspension stability, but also on the interactions with the cell. Positively charged particles appear to be the ones provoking a larger toxicity, although this has to be balanced against the fact that the internalization is favored by even small amounts of positive surface charge.

iii) The hydrophilic/hydrophobic balance of the particles is also an essential characteristic: decreasing hydrophilicity increases in turn the capacity of the opsonins to adsorb on the particles, and hence makes it easier the NP recognition by the phagocytic cells (Prijic \& Sersa, 2011).

iv) Finally, the purity is as well an issue to take care of: biocompatibility can be significantly altered by the leaching of any impurities of the particles (and this includes some stabilizers, as well). Purification at some step of the production process can be useful in this respect. 


\section{Chapter 3}

\section{Synthesis and \\ Characterization}

This chapter provides a brief description of the synthesis routes used to obtain nanocomposites as well as their morphological analysis. This will be followed by the part where nanocomposite structure, their chemical composition and magnetic characteristics will be described. 



\subsection{Design of magnetic colloids}

\subsubsection{Synthesis strategies}

The principal representatives of magnetic NPs in this field are the ferrite colloids, maghemite $\left(\gamma-\mathrm{Fe}_{2} \mathrm{O}_{3}\right)$ and magnetite $\left(\mathrm{Fe}_{3} \mathrm{O}_{4}\right)$. These two iron oxides have attracted much attention in the medical and pharmaceutical areas because of their biodegradability and biocompatibility (Laurent et al., 2008; Duran et al., 2008). Both magnetic colloids show an inverse spinel crystal structure including oxygen ions creating a close-packed cubic lattice and iron ions situated at the interstices.

The principal synthesis routes for the iron oxides preparation are: i) Physical methods: lithography and deposition from gas phase; ii) Chemical methods: sol-gel, oxidation and co-precipitation, electrochemical, hydrothermal and sonochemical decomposition reactions, flow injection synthesis, aerosol/vapour phase method; iii) Microbial methods. As an alternative, a lot of attention has also been dedicated to metal-doped iron oxides and therefore there are various synthesis procedures suggested for spinel metal ferrites $\left(\mathrm{MFe}_{2} \mathrm{O}_{4}\right.$ and $\mathrm{M}=\mathrm{Mn}, \mathrm{Zn}, \mathrm{Fe}, \mathrm{Ni}, \mathrm{Co}$, etc) (Liu et al., 2007b; Najdek et al., 2011).

The chemical method of co-precipitation. This is carried out in aqueous solution and can be done with ease, and moreover it is one of the most effective when referring to the SPIONs synthesis. It relies on chemical reactions performed in an aqueous mixture, permitting the control of the nucleation and of the process of iron hydroxide nuclei growth (Viota et al., 2007). The synthesis method consists of the precipitation of Fe (III) and Fe (II) hydroxides by combing their respective salts with a base (i.e. $\mathrm{NaOH}$ or $\mathrm{NH}_{4} \mathrm{OH}$ ). Afterwards, a precipitate (iron hydroxide) is obtained, which is later separated using magnetic decantation or centrifugation and next a concentrated base or acid is added in order to electrostatically stabilize the final solution.

The final production of iron oxide nanoparticles of appropriate size, with suitable surface characteristics and having magnetic response is determined by the stoichiometric ratio of the ferrous and ferric salts and other experimental conditions, like temperature, medium $\mathrm{pH}$, nature of salts (chlorides, nitrates etc.) or ionic strength (Massart, 1981).

The synthesis of metal-doped iron oxide nanoparticles has recently re- 
ceived a great interest. Different techniques have been suggested for manufacturing $\mathrm{MFe}_{2} \mathrm{O}_{4}$ (and $\mathrm{M}$ may be $\mathrm{Fe}, \mathrm{Mn}, \mathrm{Zn}, \mathrm{Ni}$, etc.) (Liu et al., 2007b; Najdek et al., 2011). One of them is based on a non-hydrolytic reaction of metal chloride and the iron tris-2,4-pentadioate mixed with surfactants, which moreover is later coated with DMSA. This method employs high temperature and give rise to the creation of the single crystalline iron oxide nanoparticles which present good stability (Lee et al., 2007).

\subsubsection{Stabilization methods}

The high tendency of iron to react with many oxidizing agents, makes it necessary to provide the particles with an external protection, specially when they will be in contact with water or just humid air. This surface modification will preserve its physical and chemical properties, and will also improve its stability. This can be obtained by treating the surface of the nanoparticles with a protective layer that simultaneously provides high colloidal stability, and improves their capacity for being functionalized and dispersed in water. Stabilization can be achieved in various ways:

i) The surface of the magnetic colloids can be coated using polymers or surfactants (e.g. poly(ethylene glycol)) (Lutz et al., 2006), dextran (Berry et al., 2003) or poly(vinyl alcohol) (Liu et al., 2008), or alternatively a layer containing metals (like gold (Jeong et al., 2006)), non-metals (like graphite (Seo et al., 2006)) or oxides (silica (Chastellain et al., 2004)) deposited on its surface.

ii) Magnetic colloids may be encapsulated thanks to polymers which protect them from further cluster growth and keep them separated beyond the range of attractive forces (Arias et al., 2011b; Lecommandoux et al., 2006).

iii) Iron oxide nanoparticles can also be protected by creating lipid-like coatings (such as liposomes/lipid NPs) surrounding the magnetic core (Peira et al., 2003).

The coating process can be carried out within the synthesis procedure, by adding polymers or surfactants to the route of the synthesis (Laurent et al., 2008). Depending on the chemical structure of the polymer/polyelectrolyte employed for the coating, the size of the synthesized nanoparticles can be controlled.

Most frequently, colloidal stabilization can be achieved by employing stabilizers made up of organic monomers supported by functional groups 
such as phosphate, sulphate or carboxylate (i.e. oleic acid, phosphonates) or using polymeric stabilizers like poly(etylene glycol), dextran, chitosan or poly(etylene imine)(PEI) (Reddy et al., 2012).

With respect to the vascular administration of magnetic colloids (which can be done intravenously or intra-arterially), the settling within the blood vessels may be considered insignificant, because polymer coatings in general reduce the mean density of magnetic particles, and, in addition, the drag of the blood stream can be very noticeable. The polymer structure on the surface of the nanoparticles also plays a role in determining their real hydrodynamic particle diameter and antifouling characteristics. The latter is of great importance in so far as reducing the fast blood clearance of nanoparticles after injecting them into the body. For the purpose of making easier the effective binding of polymers on the surface of the magnetic nanoparticles various molecules (like alkoxysilanes, dimercaptosuccinic acid (DMSA)) can be utilized (Fauconnier et al., 1997).

Notably, when nanoparticles are coated with polymers of hydrophilic character (like poly(etylene glycol) or dextran) or with proteins (such as lactoferrin), their plasma half-life may be enlarged up to various days. This occurs due to the characteristics provided by the polymeric shell which screens the nanoparticles detection in the body. It is worth to mention that poly(ethylene glycol) (PEG) is one of the polymers most commonly employed in nano-biomedicine, due to its biocompatibility and steric repulsion features. It is possible to find in the literature many different ways under which PEG can be bound onto magnetic nanoparticles, to mention a few: sol-gel technique (Gupta et al., 2007), polymerization in the presence of nanoparticles (Flesch et al., 2005), implantation of silane groups on the surface of the nanoparticles (Pilloni et al., 2010) among others. Besides PEG, dextran is another candidate largely used as surface modifying agent. Its capacity of generating amino groups on the surface, improves the biomolecules interactions with the nanoparticles (Corot et al., 2006; Weissleder et al., 1995).

For example, nanoshells consisting of magnetite core and polystyrene shell of very small final particle diameter $(<10 \mathrm{~nm})$ and presenting very high monodispersity have been synthesized using the PS polymerization at the magnetic nanoparticles surface (Dey, 2006). Another example can be in situ production of magnetic nanoparticles in which gelatin was employed, and which gave rise to the creation of magnetic sponge-like hydrogel materials (also known as ferrosponges) (Hu et al., 2007). These systems can easily work as a drug container for drug delivery use. The ability of the fer- 
rosponges to increase their volume, as well as their outstanding elastic and hydrophilic properties enable adequate response to an external oscillating magnetic field by fast swelling and re-swelling procedure which can be performed repeatedly. This process causes the liberation of the encapsulated antitumor drug under magnetic stimulus.

Other functional solution for nanoparticle coating is employing for this purpose inorganic metals (Prime \& Whitesides, 1991). Although there are some problems in the creation of the shell covering magnetic nanoparticles, associated,, for example, to the chemical inertness characterizing gold particles (Yu et al., 2005), coating is certainly possible, and the final iron oxide compounds with gold coating tend to be stable under conditions of acidic and neutral $\mathrm{pH}$ in aqueous solutions (Lu et al., 2007).

Non-metalic materials like graphite have been also investigated as magnetic nanoparticle coatings. It has been demonstrated that they have the ability to improve both the solubility and the stability against oxidation of FeCo nanocrystals in aqueous solutions. In addition, a graphite layer/shell onto these FeCo colloids provides them with near infrared optical absorptivity. This important feature can be useful for heat-induced treatments applied in cancer therapies (Seo et al., 2006).

For the purpose of the present investigation, it is worth mentioning that silica coatings in combination with alkoxysilane groups (e.g. 3-aminopropyltriethoxysilane or tetraethoxysilane), have also shown important benefits due to the higher stability and the simplicity of the protocol necessary to produce the surface modification of magnetic colloids (Bi et al., 2008; Lu et al., 2002). For example, a core/shell nanocompound consisting of iron oxide nanoparticle core and silica shell (10 and 10-15 $\mathrm{nm}$ are the particle size of iron oxide core and thickness of silica shell, respectively) presenting superparamagnetic behaviour and luminescent features has been prepared in a three-step process: first, silica shell is deposited onto the iron oxide cores. Later, an organic dye (tris $(2,2$ '-bipyridine) ruthenium $[\mathrm{Ru}(\mathrm{bpy})])$ was incorporated, and finally this nanostructure was coated by silica for the second time in order to provide luminescence (Ma et al., 2006).

Hybrid magnetic materials made up of magnetite in its interior and alginate/silica shell around it have been obtained via spray-drying method, and demonstrated superparamagnetic properties (Boissière et al., 2007). These carriers were incorporated within cultured fibroblasts. Moreover, a highly specific alginate degradation within cells was noticed, implying that these nanocompounds can be possible antitumor drug carriers. 
The structures obtained by encapsulating magnetic NPs in liposomes (also called magnetoliposomes) are conformed of two phospholipid layers able of being loaded with high amounts of magnetic nanoparticles. The profit of this kind of drug delivery systems is that the nanoparticle core can be within the nanostructure without being affected by possible external modifications via standard liposomal functionalization techniques (Arias et al., 2011a). Another example of magnetoliposomes includes those synthesized thanks to magnetotactic bacteria, and they are also called magnetosomes, having a typical size of $\approx 35 \mathrm{~nm}$ (Lang et al., 2007). These magnetic nanostructures are believed to play a very significant role in the future applications in biology and medicine.

\subsection{Synthesis and morphological study}

\subsubsection{Maghemite synthesis and its functionalization}

A wide variety of synthetic routes for magnetic colloids have been described (Li et al., 2006), each having its pros and cons, and it can be said that there is no synthesis which offers a general solution for all kinds of magnetic colloids. Wet precipitation and coprecipitation methods are among the most popular and having existed for longer time. For instance, by controlling the $\mathrm{pH}$ and the concentration of iron chlorides in solution, a magnetic iron oxide suspension can be obtained, containing particles with diameter around $5 \mathrm{~nm}$. A mixture of iron oxides (for example, ferrites include $\mathrm{CoFe}_{2} \mathrm{O}_{4}$, $\mathrm{NiFe}_{2} \mathrm{O}_{4}$ and magnetite) may also be prepared by coprecipitation methods starting with a stoichiometric mixed solution of salts of the desired ions. For instance, magnetite can be synthesized from solutions of $\mathrm{Fe}^{2+}$ and $\mathrm{Fe}^{3+}$ ions, as shown below:

$$
\mathrm{Fe}^{2+} \text { (aq.) }+2 \mathrm{Fe}^{3+} \text { (aq.) }+8 \mathrm{OH}^{-} \text {(aq.) } \longrightarrow \mathrm{Fe}_{3} \mathrm{O}_{4}(\mathrm{~s})+4 \mathrm{H}_{2} \mathrm{O}(\mathrm{l})
$$

Coming now to the disadvantages of the method, let us mention that the preparation of a mixture of oxides employing the coprecipitation technique is not so simple due to the fact that these ions tend to precipitate under different $\mathrm{pH}$ conditions. Likewise, $\mathrm{pH}$ regulation is crucial if one wishes to control the particle diameter, which is determined by kinetic factors. For this reason, it may happen that nanocomposites obtained via these methods often present wide size distributions and irregular morphological character- 
istics. In the case of ferrites, the Fe (II) precursor has to be protected from oxidation to obtain the mixed $\mathrm{Fe}(\mathrm{II})-\mathrm{Fe}(\mathrm{III})$ magnetic oxide.

There exists a variety of magnetic materials which can serve as magnetic cores for drug carriers, although their toxicity must be considered carefully: for instance, it is very unlikely that cobalt and chromium can find their way in the field of drug carrier design, unless special care is taken in devising protecting shells. Nevertheless, in the case of iron oxides, namely maghemite and magnetite, it has been demonstrated that they are comparatively safer than other magnetic materials, as evidenced by their wide usage in MRI, described earlier in this Thesis. Here we have chosen maghemite, as it is considered one of the most appropriate material for biomedical applications, because previous studies have shown that it is comparatively harmless (Tartaj et al., 2003).

Since maghemite is the topotactic oxidation product of magnetite, it possesses its same crystal structure, although all ions in the lattice are $\mathrm{Fe}^{3+}$. On the contrary, it also presents high magnetization, a consequence of the existence of vacancies within the crystal lattice resulting in uncompensated electron spins in the structure (McBain et al., 2008).

Their magnetic cores has to be protected from corrosion, using silica as a coating material for the purpose of this thesis. Amorphous silica is characterized, among other properties, by the presence of silanol groups (-Si-OH) on its surface providing negative surface charge for $\mathrm{pH}>3$ (Yiu et al., 2001). Silica coating onto magnetic nanocompounds may cause difficulty because of its structure, which makes it impossible the formation of a uniform layer onto the surface of maghemite. Generally, one obtains silica spheres on the SPIONs surface with particle diameter similar to that of the magnetic particles. Therefore, the overall particle diameter and its shape are difficult to control when no structure-directing agents like surfactants are employed. Usually silica coating is performed through TEOS hydrolysis under conditions of basic $\mathrm{pH}$ (8-10), like in our case, or through neutralization of silicic acid.

Procedure for the preparation of maghemite nanoparticles. $M a$ terials. Ethanol $96 \%$ solution was reagent grade from Guinama, Spain. Ammonia $\left(\mathrm{NH}_{4} \mathrm{OH}\right) 32 \%$ solution was purchased from Scharlau, Germany. Iron(III) chloride hexahydrate; iron(II) chloride; hydrochloric acid $(\mathrm{HCl})$; ammonium hydroxide; (3-aminopropyl)trimethoxysilane, APTMS $\left(\mathrm{H}_{2} \mathrm{~N}\left(\mathrm{CH}_{3}\right) 2 \mathrm{Si}\left(\mathrm{OCH}_{3}\right)_{3}\right)$; poly (diallyldimethylammonium chloride) (PDAD$\mathrm{MAC})\left(\mathrm{C}_{8} \mathrm{H}_{16} \mathrm{ClN}\right)$, (low molecular weight) $20 \mathrm{wt} \%$ in water; poly (sodium 
4-styrenesulfonate), PSS (typical molecular weight, $\mathrm{Mw}=70,000) 30 \mathrm{wt} \%$ solution in water; TEOS (tetraethoxysilane), $98 \%$ solution; sodium chloride; sodium hydroxide; iron(III) nitrate; nitric acid $\left(\mathrm{HNO}_{3}\right)$; trisodium citrate dihydrate $\left(\mathrm{Na}_{3} \mathrm{C}_{6} \mathrm{H}_{5} \mathrm{O}_{7} \cdot 2 \mathrm{H}_{2} \mathrm{O}\right)$; L-ascorbic acid $\left(\mathrm{C}_{6} \mathrm{H}_{8} \mathrm{O}_{6}\right), 99 \%$ solution; and doxorubicin $\left(\mathrm{C}_{27} \mathrm{H}_{29} \mathrm{NO} \cdot \mathrm{HCl}\right)$; trisodium citrate $(\mathrm{Na} 3 \mathrm{Ci})$; formaldehyde $\left(\mathrm{CH}_{2} \mathrm{O}\right)$; potassium carbonate $\left(\mathrm{K}_{2} \mathrm{CO}_{3}\right)$; chloroauric acid or hydrogen tetrachloroaurate $\left(\mathrm{HAuCl}_{4}\right)$; were all reagent grade from either Sigma-Aldrich (USA) or Panreac (Spain). All the chemical products and solvents were used without additional purification. Water used for prepare all of the suspensions was deionized and filtered in a Milli-Q Academic (Millipore, Spain) system.

Maghemite. Aqueous suspensions of SPIONs were obtained by Massart's coprecipitation method of ferric and ferrous chlorides in alkaline medium, and further surface oxidation by ferric nitrate was carried out (Massart, 1981). In particular, a solution of iron chlorides was prepared by dissolving $3.97 \mathrm{~g}$ of $\mathrm{FeCl}_{2}$ in $10 \mathrm{~mL}$ of $2 \mathrm{M} \mathrm{HCl}$ and $10.8 \mathrm{~g}$ of $\mathrm{FeCl}_{3}$ in $40 \mathrm{~mL}$ of Milli-Q water. Both solutions were simultaneously and rapidly added to 500 $\mathrm{mL}$ of $7.7 \mathrm{M} \mathrm{NH}_{4} \mathrm{OH}$ contained in a $1 \mathrm{~L}$ beaker, while stirring vigorously. After the sedimentation of the produced black precipitate with a magnet, the supernatant was separated and the moist precipitate was heated up to about $90^{\circ} \mathrm{C}$, while stirring on a hot plate. After about 5 min, magnetite nanoparticles were oxidized by adding to the solution $40 \mathrm{~mL}$ of $2 \mathrm{M} \mathrm{HNO}_{3}$ and $60 \mathrm{~mL}$ of $0.33 \mathrm{M} \mathrm{FeNO}_{3}$. The temperature of $90^{\circ} \mathrm{C}$ and the stirring were maintained during $1 \mathrm{~h}$. After this time the synthesis was terminated. The maghemite nanoparticle dispersion was repeatedly centrifuged and redispersed in water. All the synthesis procedures were carried out in a fume hood.

Silica Coating. A silica shell was obtained onto maghemite nanoparticles employing a modified sol-gel method described by (Salgueiriño Maceira et al., 2006), which comes from the well-known Stöber method based on the hydrolysis of TEOS (Stöber et al., 1968). First a mixture with 4.85 $\mathrm{mL}$ of $28 \% \mathrm{NH}_{4} \mathrm{OH}, 28.8 \mathrm{~mL}$ of $\mathrm{H}_{2} \mathrm{O}$ and $27.5 \mathrm{~mL}$ of $96 \% \mathrm{EtOH}$ was prepared, to which magnetic particles were added to reach a concentration of $4.8 \mathrm{mg} / \mathrm{mL}$. An ethanolic solution of TEOS $(2 \mathrm{~mL}$ of TEOS in 30 $\mathrm{mL}$ of $\mathrm{EtOH}$ ) was prepared separately and added to the maghemite suspension. Because of the necessity of avoiding aggregation of the particles, not too much TEOS should be added at once because it can result in the secondary nucleation of silica particles; the method chosen was addition of small volumes with a pipette. The hydrolysis and condensation of TEOS 
onto magnetic particles was completed in $4 \mathrm{~h}$. During all this process the solution was stirred $(210 \mathrm{rpm})$ and sonicated in a water-bath sonicator (Selecta, Spain). The formed particles were centrifuged in order to get rid of excess reactants and redispersed in $50 \mathrm{~mL}$ of MQ water. The following step was to cover the magnetic silica spheres with gold layer. Two procedures have been carried out in order to achieve it and they will be presented in the coming paragraphs.

Procedure I for the functionalization of maghemite nanoparticles Synthesis of gold seeds. Gold nanoparticles were prepared according to the standard sodium citrate reduction method (Enüstün \& Turkevich, 1963). First, $100 \mathrm{~mL}$ of an aqueous solution $\mathrm{HAuCl}_{4}\left(5 \cdot 10^{-4} \mathrm{M}\right)$ was prepared and further driven to boil. Later, $5 \mathrm{~mL}$ of $1 \%$ sodium citrate solution was added, and the mixture was stirred energetically while boiling. The synthesis was continued during 5 more minutes until appearance of a red-wine colour, which is associated to the formation of particles with small diameter. The obtained gold suspension was kept in a glass bottle in a fridge $\left(5^{\circ} \mathrm{C}\right)$ in the dark.

Deposition of gold seeds onto magnetic silica nanocompounds. In order to complete a uniform gold shell onto maghemite nanoparticles with silica coating it is required to perform a layer-by-layer deposition of cationicanionic-cationic polyelectrolytes with the aim of producing a highly charged positive substrate for gold deposition. The polyelectrolytes adsorbed sequentially were PDADMAC-PSS-PDADMAC in such a way that the chosen polymer possesses an opposite charge to that on the silica nanocompounds, as indicated elsewhere (Salgueiriño Maceira et al., 2006; Schmidt \& Thews, 1995). More precisely, we took $30 \mathrm{~mL}$ of maghemite nanocompounds with silica coating with concentration of $0.1 \% \mathrm{wt}$, and added it dropwise into $30 \mathrm{~mL}$ of an aqueous solution of polyelectrolyte $(0.05 \mathrm{~g} / \mathrm{mL})$ while it was mechanically stirred and sonicated. Before adding the solution of maghemite to a solution with polyelectrolyte, it was also sonicated for at least 20 min. The polyelectrolyte deposition was allowed to carry on for 12 hours in the same conditions. After that time the solution was centrifuged twice in a Kontron T-124 high speed centrifuge (Kontron Instruments, France) and redispersed in pure water $(30 \mathrm{~mL})$. The same procedure and concentrations were used for both polyelectrolytes. The polymer is generally adsorbed through electrostatic interactions. Magnetic silica spheres with three deposited polyelectrolyte layers in $0.2 \mathrm{M} \mathrm{NaCl}(5 \mathrm{~mL})$ were slowly added to $5 \mathrm{~mL}$ of the gold seeds solution $(0.05 \%)$. They were left in contact during 


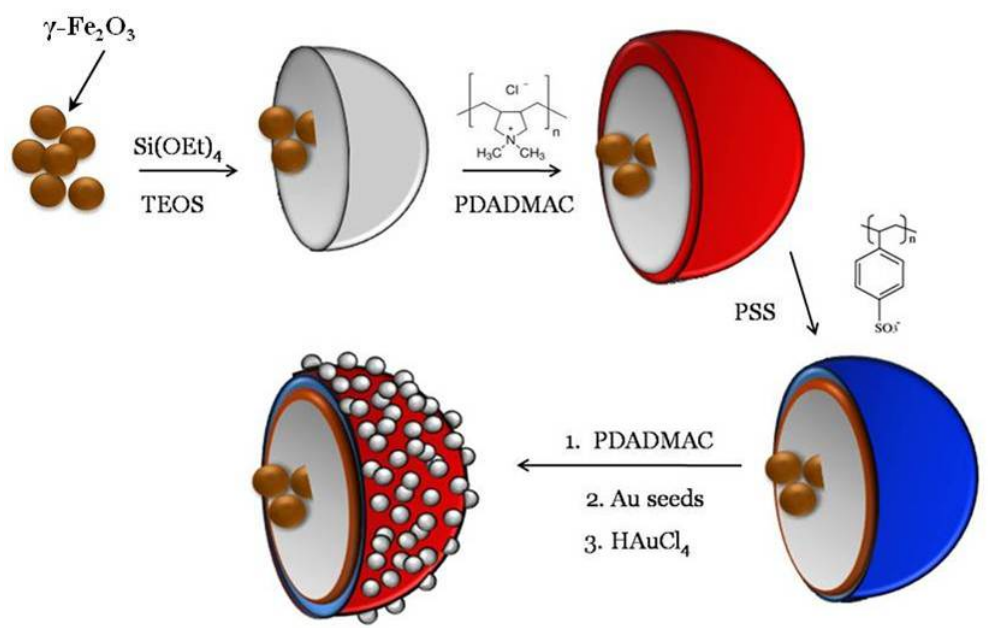

Figure 3.1: Schematic illustration of the procedure I.

$20 \mathrm{~min}$. After this time the solution was centrifuged three times and the particles were redispersed in $10 \mathrm{~mL}$ of $\mathrm{MQ}$ water.

Production of the gold shell. The following step after deposition of gold nanoparticles on silica-coated maghemite with three polyelectrolyte layers is the growth of the uniform gold shell. The gold seeds attached to the surface of silica-coated maghemite were exploited to template the growth of the continuous gold shell. This was achieved by reducing small amounts $(10 \mu \mathrm{L})$ of $5 \cdot 10^{-4} \mathrm{M} \mathrm{HAuCl}_{4}$ with small amounts of ascorbic acid $(10 \mu \mathrm{L}$, $\left.0.34 \cdot 10^{-3} \mathrm{M}\right)$ in aqueous solution of silica-coated maghemite with gold seeds deposited. For the resulting particles see Fig. 7.1.

Procedure II for the functionalization of maghemite nanoparticles Surface treatment with APTMS. The silica-coated iron oxide nanoparticles were treated with APTMS as described elsewhere (Pham et al., 2002). Briefly, $10 \mathrm{~mL}$ of silica-coated maghemite nanoparticles with particle concentration $6.5 \cdot 10^{11}$ particles $/ \mathrm{mL}(5.4 \mathrm{mg} / \mathrm{mL})$ was mechanically stirred and sonicated at the same time during 5 minutes. Afterwards, $5 \mu \mathrm{L}$ of APTMS was added to the solution, and the resulting solution was left under continuous mechanical stirring and sonication during $3 \mathrm{~h}$. After this time, the solution was cleaned three times by successive cycles of centrifugation at $14000 \times \mathrm{g}$ during $10 \mathrm{~min}$, followed by redispersion in water. In the final cycle ethanol was used as suspending liquid.

Gold seeds deposition. Gold nanoparticles were obtained by the reduc- 
tion method of chloroauric acid with $\mathrm{NaBH}_{4}$ reported in (Busbee et al., 2003). As a first step, $0.5 \mathrm{~mL}$ of a $0.1 \mathrm{M} \mathrm{NaBH}_{4}$ solution was prepared and cooled down inside a vessel with ice. Next, $0.5 \mathrm{~mL}$ of $0.01 \mathrm{M} \mathrm{HAuCl}_{4}$ was mixed with $0.5 \mathrm{~mL}$ of $0.01 \mathrm{M} \mathrm{Na}_{3} \mathrm{Ci}$ and added to $18 \mathrm{~mL}$ of distilled water. The following step is adding energetically the $0.5 \mathrm{~mL}$ of the cooled $\mathrm{NaBH}_{4}$ solution, while stirring in a vigorous manner at room temperature. This results in the reduction of $\mathrm{Au}^{3+}$ ions to neutral gold atoms. After a few seconds, when the color of the solution changes to pink, stirring was stopped and the synthesis was left to stay for $2 \mathrm{~h}$. The resulting gold concentration was $2.3 \cdot 10^{13}$ particles $/ \mathrm{mL}(50 \mathrm{mg} / \mathrm{L})$. In order to deposit the gold seeds onto magnetic silica nanocompounds, the nanoparticle dispersion with the previously deposited APTMS layer $(1 \mathrm{~mL})$ was added dropwise to the gold seed solution $(7 \mathrm{~mL})$ under energetic stirring in ultrasounds bath. At last, the solution was left $2 \mathrm{~h}$ under mechanical stirring and finally it was cleaned as described previously, but redispersing in $\mathrm{MQ}$ water.

Formation of the gold layer. First, $25 \mathrm{mg}(0.18 \mathrm{mmol})$ of $\mathrm{K}_{2} \mathrm{CO}_{3}$ was dissolved in $100 \mathrm{~mL}$ of water. After a few minutes of vigorous stirring, $1 \%$ $\mathrm{HAuCl}_{4}$ solution $(1.5 \mathrm{~mL})$ was added. It was stirred for $30 \mathrm{~min}$ and later $8 \mathrm{~mL}$ of this solution was collected. Then, $600 \mu \mathrm{L}$ of nanocompounds with gold seeds deposited was injected to $8 \mathrm{~mL}$ volume while stirring, followed by addition of $40 \mu \mathrm{L}$ of formaldehyde. When the color of the solution turns violet, the gold layer is considered completed. The particles were finally centrifuged and redispersed in water as described (Pham et al., 2002).

Summarizing, magnetic maghemite cores have been encapsulated into a silica shell, and further their surface was treated with a silanizing agent (APTMS) possessing amine surface groups. The final amine groups act like attachment sites for gold seeds which subsequently perform a function as nucleation sites for the coalescence of the fine layer of gold (See Fig. 7.2). The inner magnetic $\gamma-\mathrm{Fe}_{2} \mathrm{O}_{3}$ core furnish the nanocompounds with magnetic properties, while on the contrary particle diameter, shape, surface characteristics, drug loading efficacy, and biological fate, the most significant properties governing the application as drug delivery systems, both in vitro and in vivo, are generally dependent on the coatings.

\subsubsection{Morphological study}

Microscopic observations are essential to assess the resulting nanostructures as suitable for biomedical applications. For the purpose of this work highresolution transmission electron microscopy (Philips STEM CM20, Netherlands) has been used in order to obtain pictures of the synthesized nanos- 


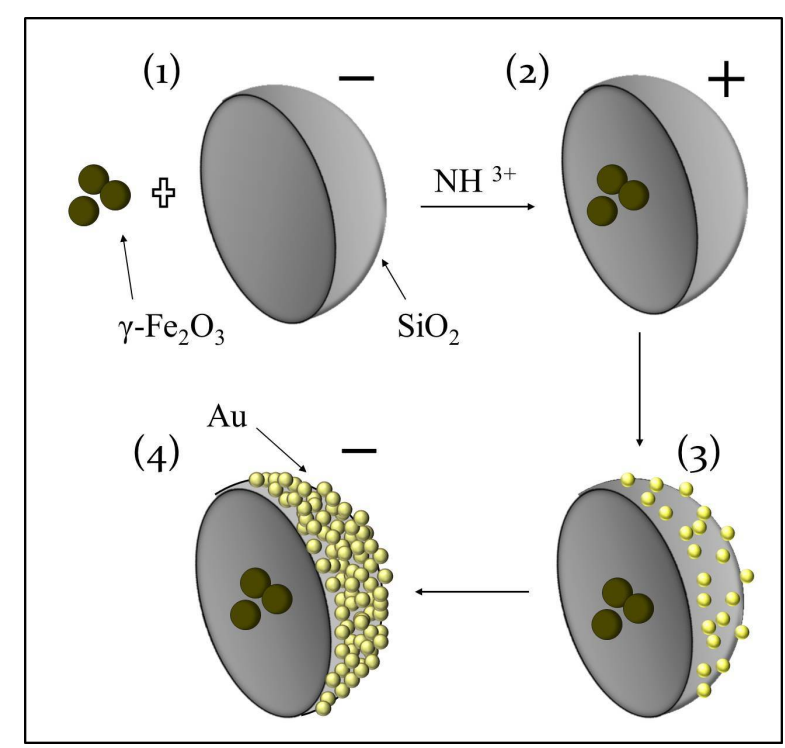

Figure 3.2: Schematic illustration of procedure II. 1) The coating process of maghemite with silica; 2) Surface treatment with APTMS; 3) Deposition of gold seeds; 4) Growth of the final gold layer.

tructures. HRTEM photos (Fig. 7.3) displays the morphology of $\gamma-\mathrm{Fe}_{2} \mathrm{O}_{3}$. It can be noticed that the maghemite nanoparticles are almost spherical and rather monodisperse. The particle diameter determined from HRTEM micrographs in a direct manner is approximately of $15 \mathrm{~nm}$ with a standard deviation of $5 \mathrm{~nm}$, which, as will be presented below, is comparable to the measured particle diameter by means of light scattering determinations. Furthermore, it can be observed that all maghemite particles were satisfactorily dispersed and no aggregation was noticed. Let us underline that the described well-defined and reproducible synthetic routes are fundamental for strict control of features when dealing with biomedical applications.

Particle sizing was performed not only based on the microscopic study, but also through in situ static and dynamic light scattering (photon correlation spectroscopy). These experiments were carried out, respectively, in a Mastersizer 2000 and in a PCS 3700 (both from Malvern Instruments, England). The former device works on the basis of Mie theory for the estimation of size distributions from the angular variation of the scattered intensity, while the PCS 3700 is fundamented on dynamic light scattering (DLS). Details of the techniques can be found in the wide existing literature (Bantle et al., 1982; Brown, 1993; Kerker, 1969; Pecora, 1985). 

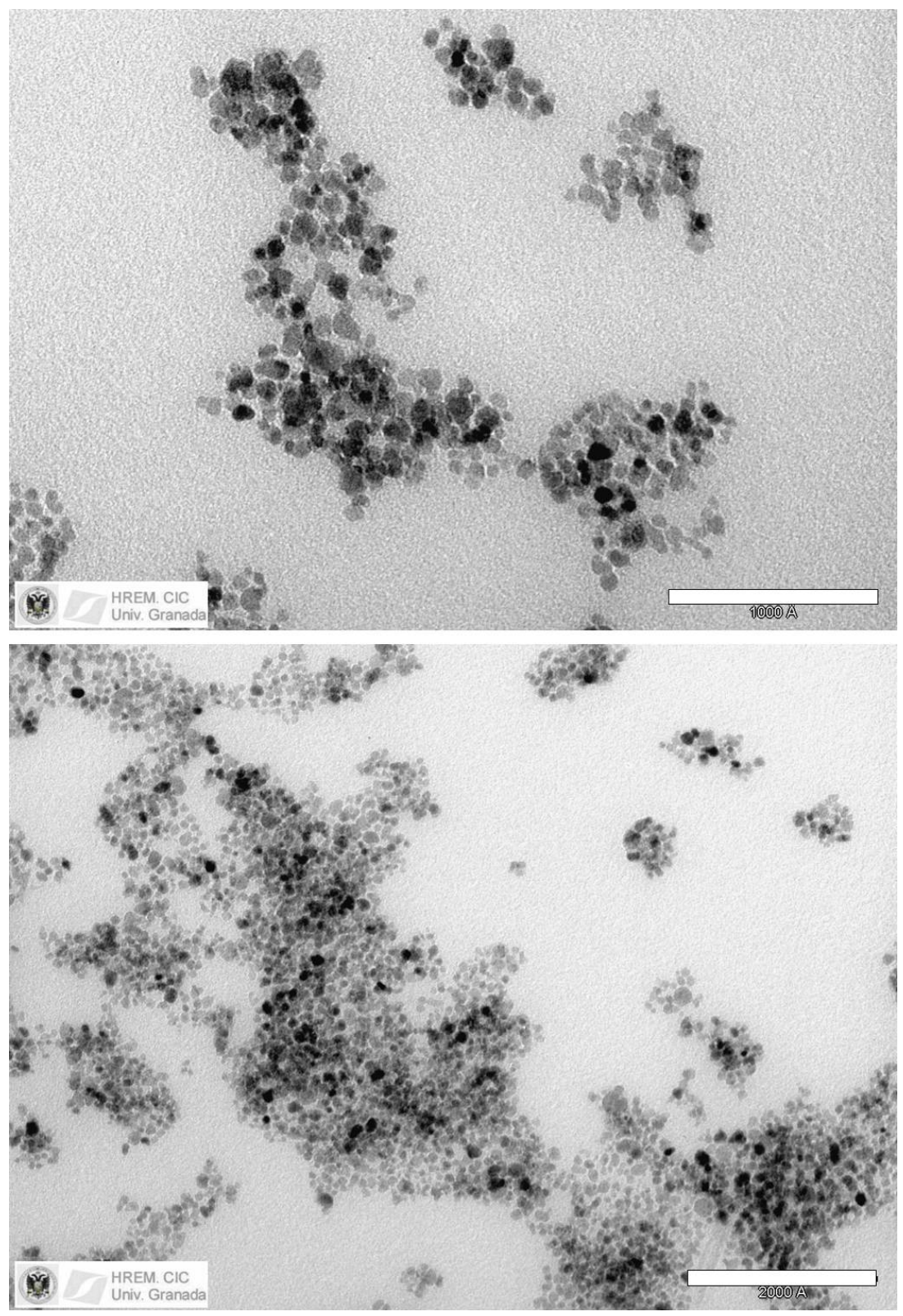

Figure 3.3: TEM pictures of synthesized maghemite particles. Scale bars $100 \mathrm{~nm}$ (top) and $200 \mathrm{~nm}$ (bottom). 


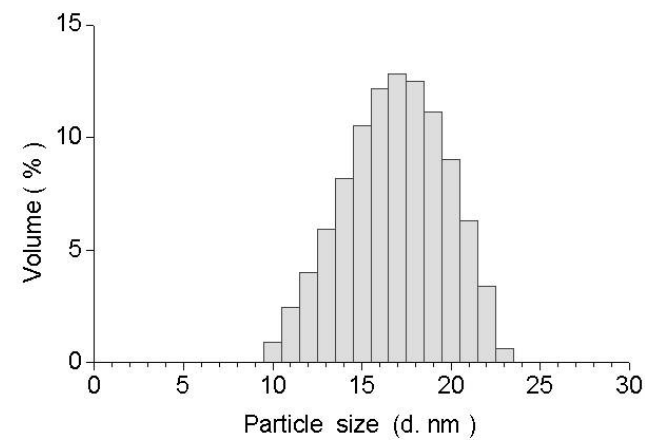

Figure 3.4: Size distribution (by volume) histogram for synthesized maghemite obtained from static light scattering.

The average particle diameter of the synthesized iron oxide nanoparticles was $17 \pm 4 \mathrm{~nm}$, as evaluated by the static light scattering technique. Fig. 3.4 shows the size distribution found: the principal population has an average size of about $15 \mathrm{~nm}$, but the existence of some aggregates cannot be rejected. The polydispersity of the maghemite sample is not very marked. As already mentioned, the benefit of employing maghemite nanoparticles generally is connected with its chemical purity and, above all, geometrical homogeneity. This fact is verified by taking into account that the particle size distribution is quite narrow.

The subsequent step in the preparation of our final nanocomposites includes the process of coating of superparamagnetic iron oxide nanoparticles with silica in order to isolate the maghemite core from the surrounding environment. However, maghemite surface has an accented affinity for amorphous silica, the coating procedure may be difficult because of aggregation of maghemite nanoparticles. Due to this fact, the magnetic core is composed of several individual particles of maghemite in the majority of the cases. Fig. 7.4 illustrates these results: the presence of more than one maghemite nanoparticle inside the silica shell can be noticed. Dynamic light scattering measurements of dilute solutions of silica-coated maghemite nanoparticles gave an average hydrodynamic particle diameter of $202 \pm 14 \mathrm{~nm}$.

Procedure I for the funtionalization of maghemite nanoparticles As described earlier, the first procedure for complete gold shell formation is based on the successive deposition of three polyelectrolytes. This step can be achieved thanks to the Layer-by-Layer technique which permits to coat the solid silica shell with various layers of polyelectrolytes thanks to electrostatic self-assembly. Light scattering data reported by (Caruso et al., 


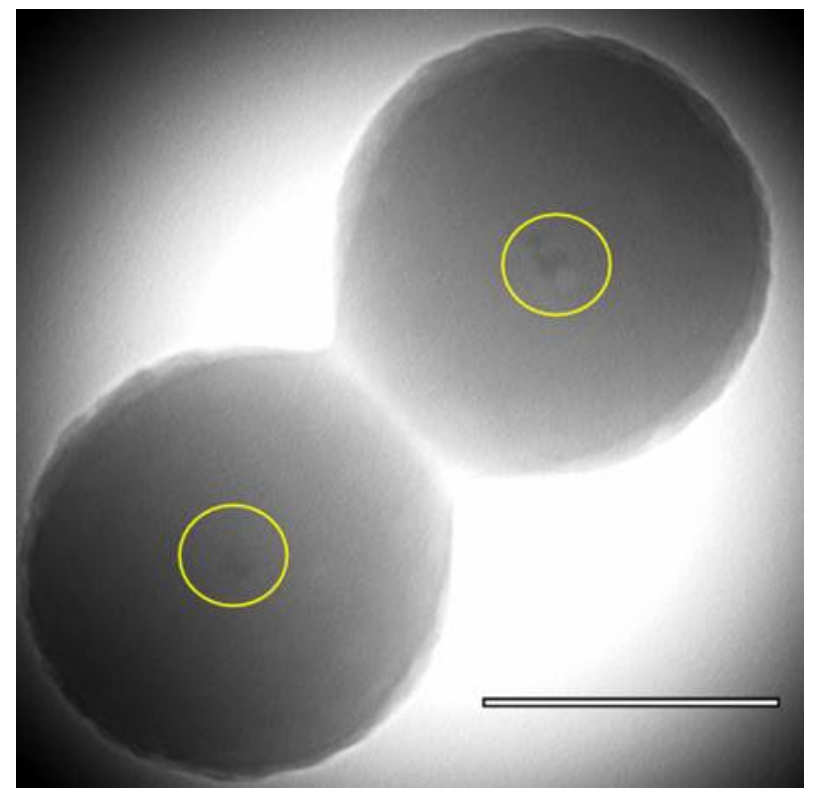

Figure 3.5: TEM picture of silica-coated maghemite nanoparticles. Bar length - $200 \mathrm{~nm}$. The color circles indicate magnetic nuclei.

1999) (Tab. 3.1) demonstrate that the average thickness of successive layers is approximately $1.5 \mathrm{~nm}$ in the case of PDADMAC/PSS polyelectrolytes as in our synthesis. The control that the technique allows to the nm scale is very noticeable.

Fig. 3.6 exhibits an example of the synthesized gold seeds and their particle size distribution. From various images like this, and also from dynamic light scattering data (Fig. 3.6B) it can be determined that the particle diameter of these gold seeds is around $10 \mathrm{~nm}$.

DLS measurements were performed as well in order to determine the hydrodynamic diameter of the samples, obtaining $260 \pm 30 \mathrm{~nm}$ and 202 $\pm 16 \mathrm{~nm}$ for polymers (PDADMAC/PSS/PDADMAC)-coated magnetic silica nanospheres and final nanocomposites with gold layer respectively. Note that the addition of gold particles provokes the partial compression of the soft polyelectrolyte layer on which they are deposited. Fig. 3.7 displays an HRTEM image of the final nanocomposites obtained following the first synthesis route.

Procedure II for the functionalization of maghemite nanoparticles Fig. 3.8 presents a size distribution of synthesized small colloidal gold em- 


\begin{tabular}{ccc}
\hline Film thickness $(\mathrm{nm})$ & & \\
\hline Number of layers & PAH/PSS & PDADMAC/PSS \\
\hline 1 & 1.2 & 1.4 \\
3 & 3.4 & 3.3 \\
5 & 5.6 & 6.6 \\
7 & 8.4 & 8.5 \\
9 & 11.5 & 11.7 \\
11 & 15.0 & - \\
15 & 24.8 & - \\
21 & 33.9 & - \\
\hline
\end{tabular}

Table 3.1: Thickness of polyelectrolyte multilayer films assembled onto polystyrene latexes; PAH = polyallyamine hydrochloride), PSS = poly(styrenesulfonate), PDADMAC = poly(diallydimethylammonium chloride); Values were derived from SPLS data employing the Rayleight-DebyeGans theory and a refractive index of 1.47 for the polymer layers (Caruso et al., 1999).

A

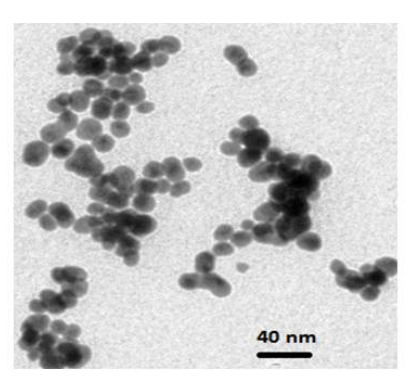

B

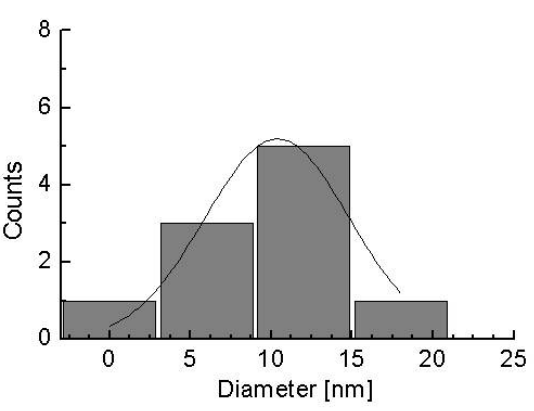

Figure 3.6: TEM image (A) and dynamic light-scattering measurements (B) of gold NPS synthesized by standard sodium citrate reduction method and used further as seeds to form the gold shell. 


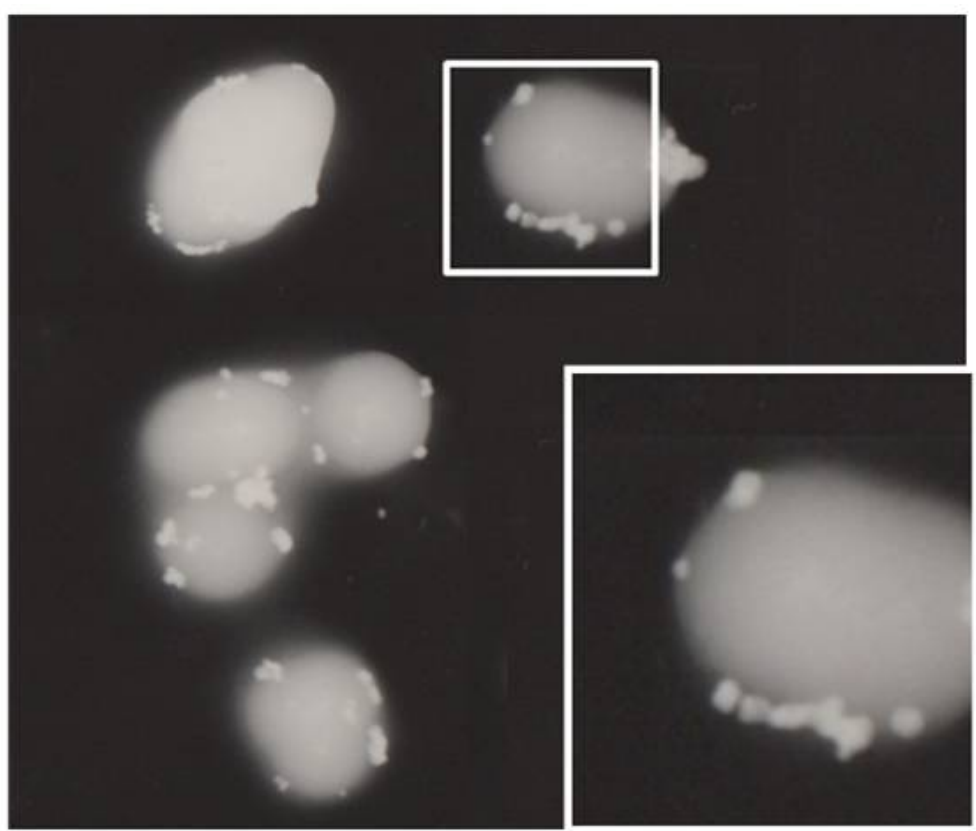

Figure 3.7: Final nanocompounds obtained using the first procedure. Photo of the gold shell creation on the silica-coated maghemite nanospheres by occupying the voids between gold nanoparticles deposited onto the surface of the maghemite covered with silica. 


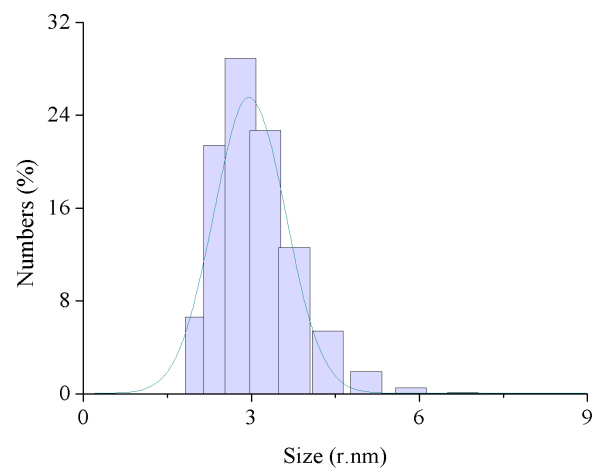

Figure 3.8: Size histogram of gold seeds synthesized by the reduction of chloroauric acid with $\mathrm{NaBH}_{4}$.

ployed which will serve as nucleation sites for the gold layer growth. Fig. 7.5 and displays typical HRTEM images of nanocompounds coated with gold seeds (A, B) and with a final thin gold layer on the surface of magnetic silica spheres $(\mathrm{C}, \mathrm{D})$, created utilizing the described second procedure. These photos show that the resulting particle size is approximately $200 \mathrm{~nm}$ and that the gold coating is not smooth but uninterrupted.

Not only it is significant that the particle diameter is in the accurate range, but on top of that the synthesis route of monodisperse nanocomposites is essential for achieving reliable cytotoxicity and in vitro results which permit to be repeated. Dynamic light scattering measurements are excellent for yielding information about the particle size distribution in aqueous media, these working as an indirect test of the stability of the suspensions. Average hydrodynamic sizes for nanocomposites are not influenced by either sedimentation or aggregation (Chapter 6). 


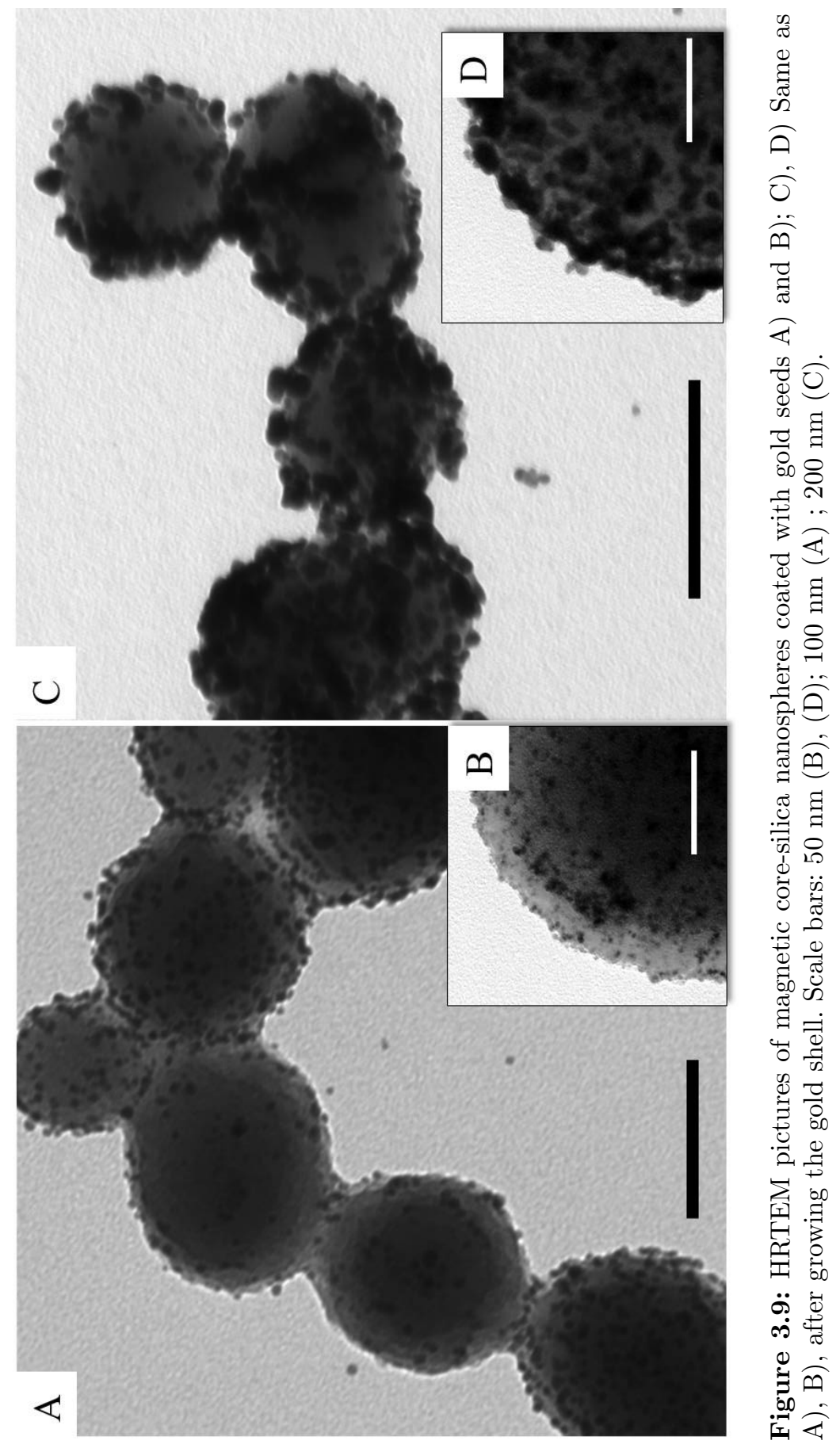




\subsection{Structure and chemical composition}

\subsubsection{X-ray Powder Diffraction (XRD)}

The XRD evaluation of a maghemite $\left(\gamma-\mathrm{Fe}_{2} \mathrm{O}_{3}\right)$ sample in powder was performed utilizing a Bruker D8 Advance (USA) powder diffractometer using $\mathrm{Cu}-\mathrm{K} \alpha$ radiation. The parameters selected for the determinations were $2 \theta$ steps of $0.02^{\circ}, 8 \mathrm{~s}$ of counting per step, and $2 \theta$ range from $3^{\circ}$ to $80^{\circ}$ at room temperature. The instrument is available at the University of Granada (Scientific Instrumentation Centre).

The X-ray diffraction pattern of the obtained magnetic iron oxide colloids is presented in Fig. 3.10. It corresponds to that of the standard maghemite but shows peaks which match with two iron oxides, namely, maghemite $\left(\gamma-\mathrm{Fe}_{2} \mathrm{O}_{3}\right)$ and magnetite $\left(\mathrm{Fe}_{3} \mathrm{O}_{4}\right)$. All X-ray diffraction peaks may be ascribed to the iron oxide inverse spinel structure. As maghemite is usually produced by oxidation of magnetite and the crystallographic (as well as magnetic) characteristics of maghemite are very similar to those of magnetite, it is very hard to differentiate between magnetite and magnetite/maghemite phases since XRD spectra are not dissimilar enough in that aspect. In our case, the six XRD peaks, with Miller indices (220), (311), (400), (422), (511), (440), agree with those of the reference data for maghemite (Greaves, 1983; Haas, 1965; Morales et al., 1994; Shmakov et al., 1995; Wells, 1975) in more than 58 \% (see Fig. 3.11), indicating that the creation of magheite phase is most likely. Therefore, the presence of maghemite nanoparticles is confirmed, although it may be not fully crystalline, as demonstrated by the slightly broad XRD lines, indicative of the production of very small particles, or of the presence of impurities and structural/crystallographic disorder to a large extent. The width of the (311) peak suggests that the average crystallite diameter of the obtained iron oxides (Mikhaylova et al., 2004). The $\gamma-\mathrm{Fe}_{2} \mathrm{O}_{3}$ nanoparticles were identified as well by confirmation of the main peak position. This peak (indices 311 ) was found at $35.68^{\circ}$ slightly shifted (Fig. 3.10) in comparison with the standard $\theta$ positions for maghemite - $35.631^{\circ}$ (JCPDS 39-1346) and magnetite $-35.423^{\circ}$ (JCPDS 19-629) (Feltin \& Pileni, 1997). This is probably due to particle oxidation, although the sample may additionally contain some small amounts of impurities like hematite or goetite. 


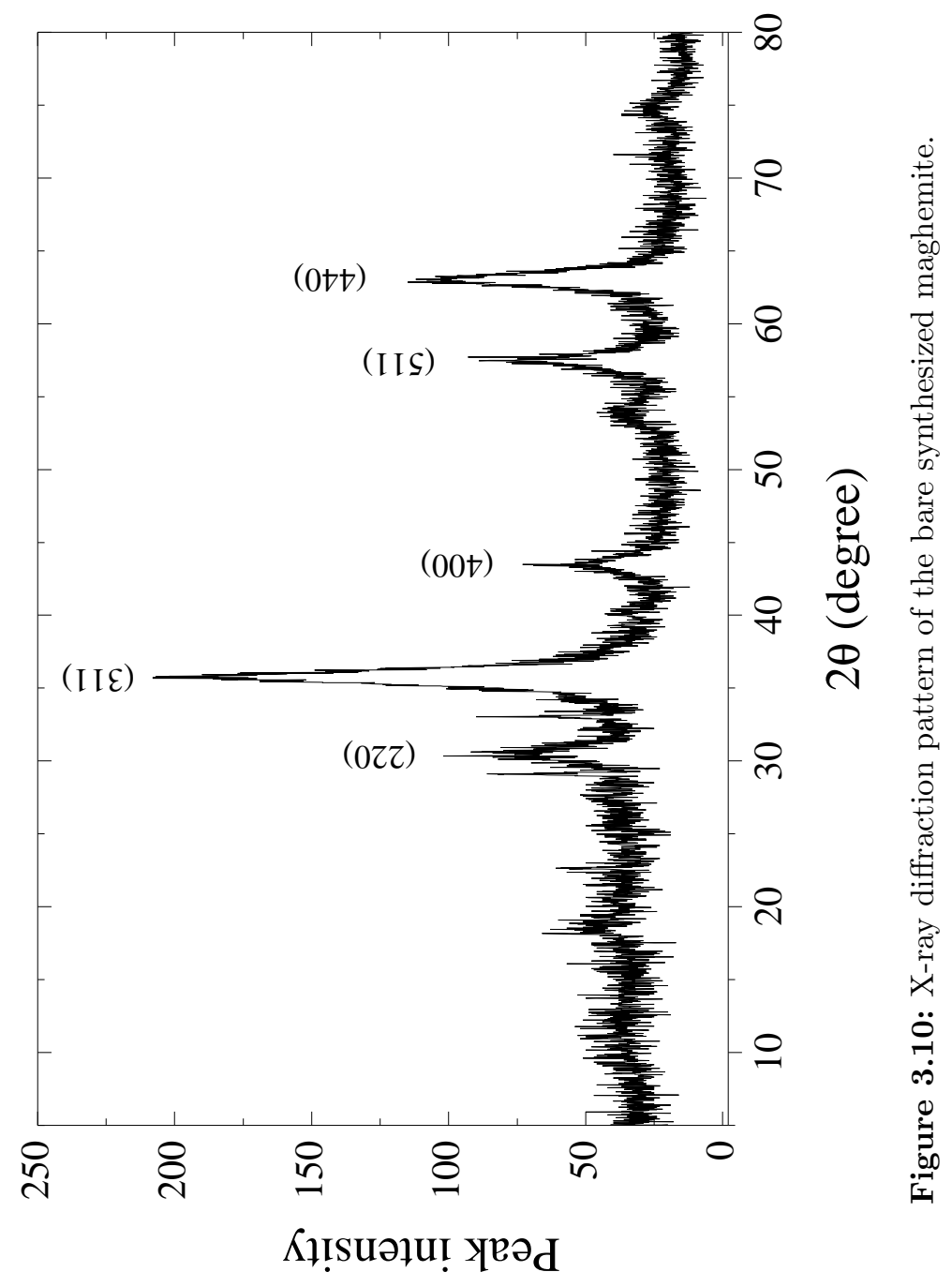




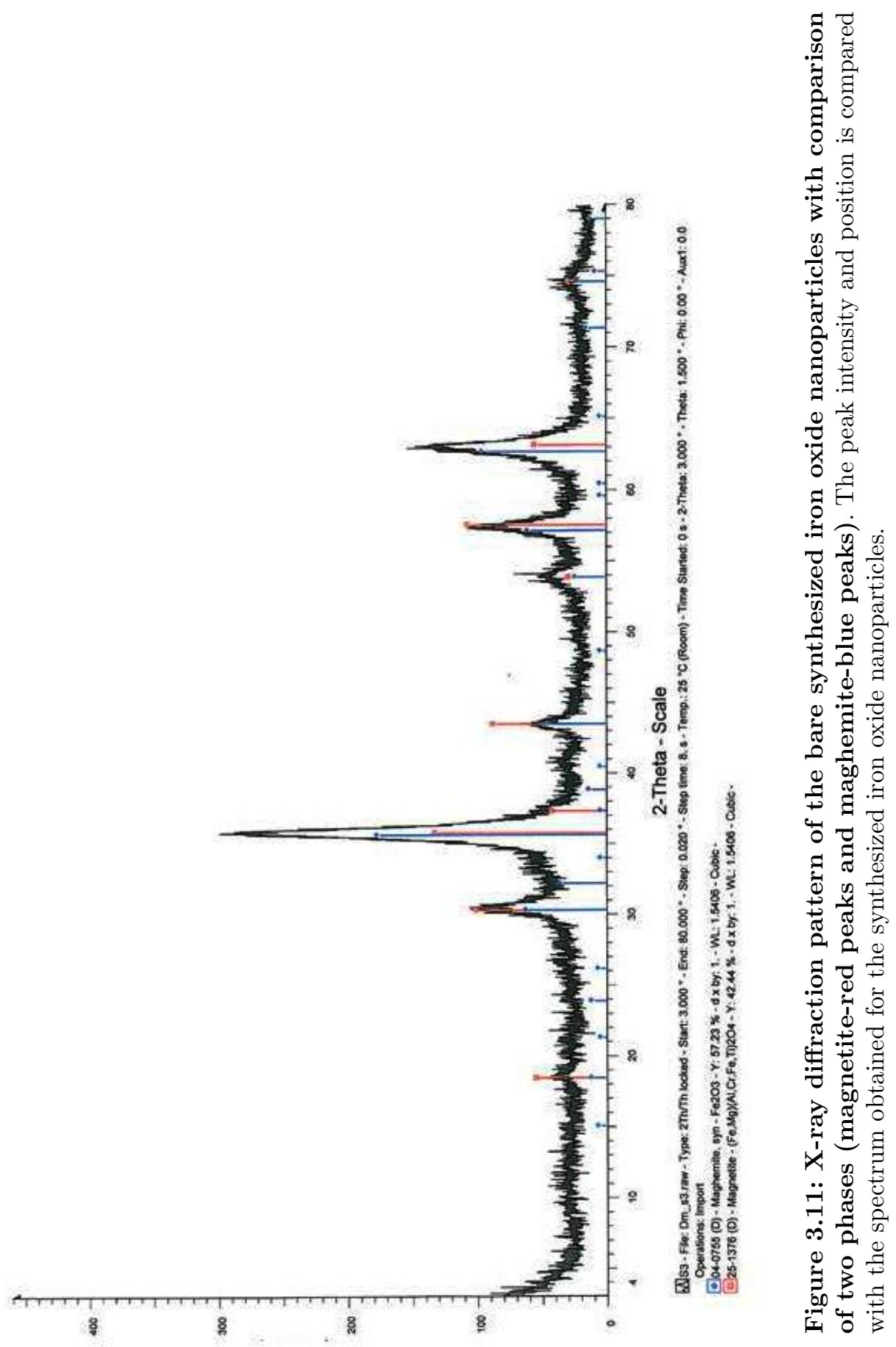




\subsubsection{X-ray Photoelectron Spectroscopy (XPS)}

$\mathrm{X}$-ray photoelectron spectroscopy is a nondestructive technique for investigating the electronic structure of atoms, molecules, and solids. In early studies, XPS was employed for the spectroscopy of atoms and molecules existing in the gas phase, excited with monochromatic ultraviolet light (He I: $21.2 \mathrm{eV}$, He II: $40.8 \mathrm{eV}$ ) and further for spectroscopic studies of solid materials by stimulation with soft X-rays $\left(\mathrm{Al}-\mathrm{K}_{\alpha}: 1486.7 \mathrm{eV}, \mathrm{Mg}-\mathrm{K}_{\alpha}: 1253.6\right.$ eV) (Siegbahn, 1967). The technique was improved by Kai Siegbahn (Nobel Prize in Physics 1981) and was called ESCA (or Electron Spectroscopy for Chemical Analysis). In the case of the XPS method the radiation employed is a monochromatic X-ray beam $\left(\mathrm{Al}-\mathrm{K}_{\alpha}, \mathrm{Mg}-\mathrm{K}_{\alpha}\right)$ or He I, He II, synchrotron radiation or laser light, and it is utilized for separation of electrons from atoms, molecules and solid materials. The momentum and energy of the photoelectrons excited from the sample permit to obtain direct data about electronic structure of this sample. With the help of XPS, the shell structure of atoms may be determined. Let us mention that when dealing with condensed matter the XPS technique is extremely surface sensitive due to the fact that only photoelectrons from a fine surface layer are given out without any loss. The escape depth $\lambda$ ranges from 2 to $20 \AA$, depending on the kinetic energy $\left(E_{k i n}\right)$ of the photoelectron. $\lambda$ may be estimated with the help of the so-called universal curve that is characterized by a minimum of approximately 2 Åat kinetic energies $E_{k i n} \cong 40 \mathrm{eV}$ (greatest surface sensitivity).

Once the electron is extracted out of the inner layer of the atom, this becomes ionized. The energy balance in the process reads:

$$
E_{B}=h \nu-\Phi-E_{k i n}
$$

where $h \nu$ is the energy of the photon, $\mathrm{E}_{B}$ represents the binding energy of the electron, and $\mathrm{E}_{k i n}$ is its kinetic energy. If we can determine the photon energy $(\mathrm{h} \nu)$, the binding energy may be evaluated for all core electrons for which $\mathrm{E}_{B}<\mathrm{h} \nu-\Phi$. Fig. 3.12 shows the typical excitation processes in solid materials in an XPS process:

a) emission from core levels

b) Auger processes

c) emission from the valence band

d) secondary electron excitation and energy losses

Moreover, peaks are found in well-specified positions with respect to photo-emission lines, for instance Auger electrons or satellite peaks, re- 


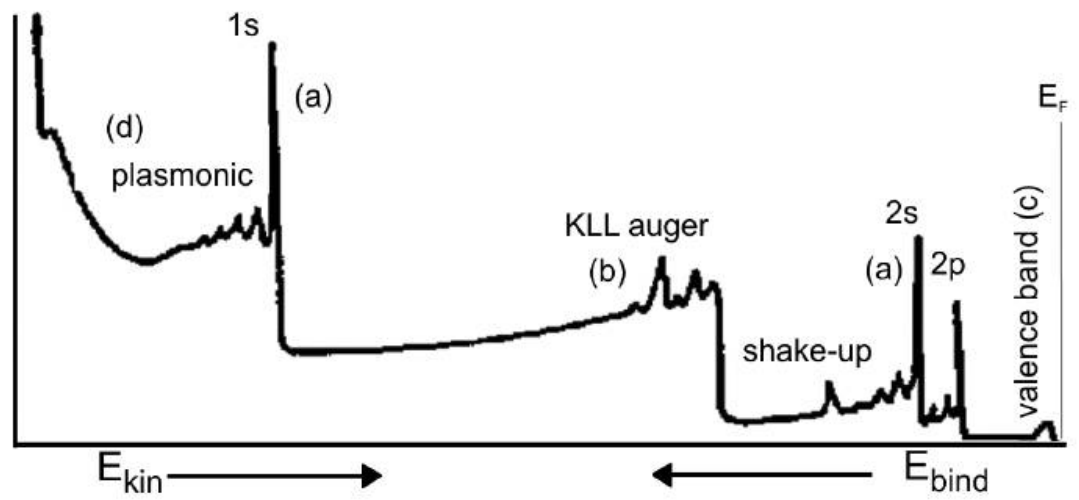

Figure 3.12: Overview of the XPS process in a solid.

sulting from plasma excitation, that is, collective oscillations of conduction electrons.

There are various inputs to the linewidth of a photo-emission line that can be found. Among others exist: the lifetime width of the ultimate state, the width of the inciting X-ray line, the thermal distribution by interacting with photons and the energy resolution of the electron analyzer. Normally the width of the $\mathrm{Mg}-\mathrm{K}_{\alpha} \mathrm{X}$-ray line is approximately $<1 \mathrm{eV}$ and the energy resolution is of order of $1 \mathrm{eV}$ (at the kinetic energy $\approx 1000 \mathrm{eV}$ ).

Methodology. XPS measurements were carried out in an X-ray photoelectron spectrometer Kratos Axis Ultra-DLD (UK), using a monochromatic K $\alpha$ x-ray source. The settings for the wide survey scan were: energy range $=1200-0 \mathrm{eV}$, pass energy $=160 \mathrm{eV}$, step size $=1 \mathrm{eV}$, dwell(s) time $=0.1 \mathrm{~s}$, number of sweeps $=1$. In case of the Fe $2 \mathrm{p}$ region, and also for the $\mathrm{O}$ 1s region, for the high-resolution spectra measurements, we used an energy range of $50-20 \mathrm{eV}$ and a step size of $0.1 \mathrm{eV}$.

Results. The data obtained with our particles are presented in Fig. 3.13A (wide general spectrum) and Fig. 3.13B (iron bands). In accordance with published data (Grosvenor et al., 2004; Martinez et al., 2007; Koleva et al., 2009 ), the peaks found at $711.5 \mathrm{eV}$ and $725 \mathrm{eV}$ match, respectively, the $\mathrm{Fe}$ 2 p $1 / 2$ and $\mathrm{Fe} 2$ p $3 / 2$ band (categorized as the $\mathrm{Fe}^{2+}$ state of $\mathrm{Fe}_{3} \mathrm{O}_{4}$ ). These, together with $717 \mathrm{eV}$ satellite peak, permit to differentiate unmistakably that the sample only contains $\mathrm{Fe}^{3+}$ states, and therefore our nanoparticles are mainly maghemite (Grosvenor et al., 2004; Koleva et al., 2009; Martinez 

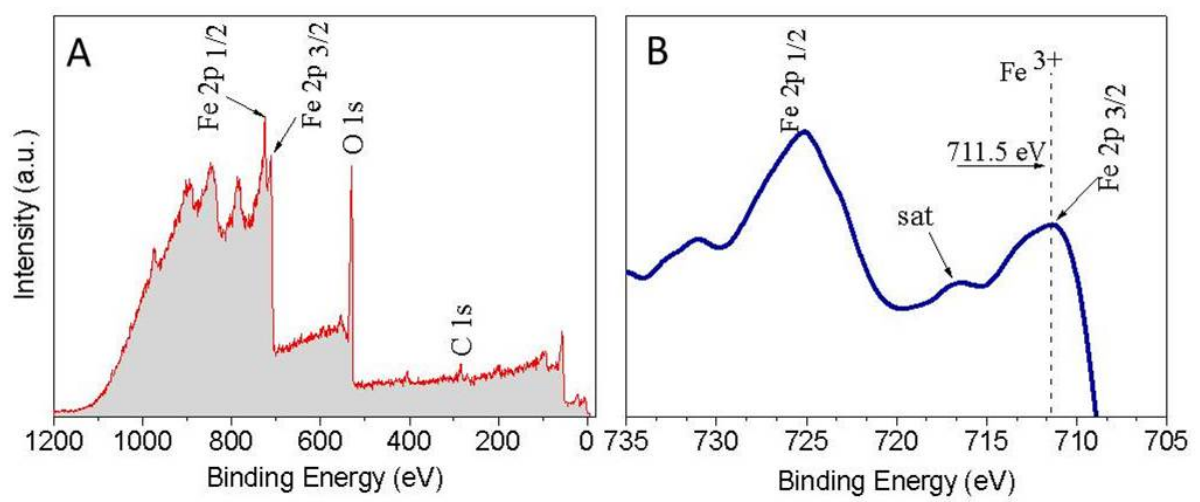

Figure 3.13: The XPS wide spectrum (A) and the Fe 2p spectrum (B) obtained for the iron oxide nanoparticles powder sample.

et al., 2007). In addition, the sample peak centred at $530 \mathrm{eV}$ exhibited in the $\mathrm{O}$ 1s region (Fig. 3.13A) further confirms that conclusion (Temesghen \& Sherwood, 2002). 


\section{Chapter 4}

\section{Electrokinetic properties}

Because the systems designed are in fact surface modifications of previously existing particles, it can be proposed that those techniques devised for the evaluation of surface properties are well suited for performing a follow-up of the efficiency of the coatings carried out. This will be shown in the present chapter. 

It is a virtually universal fact that when a solid particle is placed in contact with a polar medium (an aqueous solution in our case) surface groups ionization or unequal adsorption of ions from solution will provoke the appearance of a surface charge on the solid. The phenomenon acquires the utmost importance when we are dealing with very small dimensions of the dispersed solid material, since in this case surface electrostactics becomes a determinant characteristic of the system. In fact, electrostatic interactions may control most macroscopic properties of the disperse system (stability or coagulation, rheology, particle size, color and turbidity, field of application and so on). Looking at the interface in more detail, we must consider that the surface charge thus generated must be compensated for by some countercharge in the solution in roder to guarantee electroneutrality. Ions in solution will tend to approach the interface if they are oppositely charged to the latter (counterions), whereas they will be repelled otherwise (coions). Increased (decreased) concentrations of counterions (coions) will tend to be opposed by diffusion, and a steady state distribution of ions known as (Electrical Double Layer $(E D L)$ ) will be produced. Its structure and our ways to obtain information on it are described below.

\subsection{Description of the electric double layer}

Even though its name contains the word "double ", the EDL structure can be rather complex even in the equilibrium state. Fig. 4.1 schematically presents the various regions which can be distinguished in every EDL. More details can be found in classical references like the textbooks by Hunter and by Lyklema ((Lyklema, 1991; Hunter, 1981, 1987)). Next to the solid surface (or on the surface itself), charges determining the surface charge $\left(\sigma_{0}\right)$ are found, and the potential on this plane (at the solid surface) is $\psi_{0}$. In direct proximity to the first plane, ions which may experience specific adsorption might be found. One can assume that these ions may not possess hydration shell yet (in the direction of the surface), and therefore, the space between them and the solid surface is in the order of the radius of a dehydrated ion. This plane is characterized by the charge density $\sigma_{i}$. The ions found in this plane, which cause the potential $\psi_{i}$, are interacting with the surface not only via electrostatic interactions, but also in many cases they can succeed in facing up electrical repulsions and may be able to raise the positive charge of the surface which was previously positive. It is commonly said that the 
lacking interactions are of chemical nature. However, this is may not be true, since there exist situations in which in addition to (or in place of) the creation of chemical (covalent) bonds, van der Waals attractions, hydrogen bonds or even hydrophobic-hydrophilic forces may be responsible for this loosely named specifically adsorbed ions. It is admitted that it can located at some specific distance $\beta_{i}$, defining the inner Helmholz plane (IHP) - at the distance $\beta_{i}$ in Fig. 4.1) (Delgado \& Arroyo, 2002; Delgado et al., 2007).

More distant from the solid surface, at a distance $\mathrm{x}=\beta_{d}$ and further than that point, ions which experience only electrostatic interactions and diffusion are placed. Due to the fact that the character of their interactions with the solid surface is not so strong, they can be also under influence of solvent (collisions with solvent molecules) hence they are, actually, spread out diffusively in the solution in contact with the solid surface. These ions do possess a hydration shell what allows them to move close to the surface, up to the distance $\mathrm{x}=\beta_{d}$, which is the hydrated ion radius. The plane found at a distance $\mathrm{x}=\beta_{d}$ is named the outer Helmholtz plane (OHP). The volume between $\mathrm{x}=0$ and $\mathrm{x}=\beta_{d}$ represents a charge-free region between the solid surface and the location of hydrated counterions known as Stern layer or inner part of the double layer, or dense part of the double layer. The OHP determines the beginning of the diffuse layer or Gouy-Chapman layer.

As mentioned before, $\sigma_{0}$ represents the charge density at the solid surface, $\sigma_{i}$ characterizes the charge density at the inner Helmholtz plane and $\sigma_{d}$ is the charge density within the diffuse layer. According to the electroneutrality condition of the system:

$$
\sigma_{0}+\sigma_{i}+\sigma_{0}=0
$$

As for the ions in the EDL, it is usual to differentiate between so-called indifferent and specifically adsorbing ones. The first type of ions is adsorbed solely by means of Coulomb forces and do not adsorb preferentially on an uncharged surface. On the contrary, specifically adsorbed ions are characterized by the above mentioned chemical or specific affinity for the surface. It has been proposed by (Lyklema, 1991) to rather use the denomination of surface ions to ions which are components of the solid, and therefore they are considered to sit on the surface. Protons and hydroxyl ions are also rated among these surface ions. 


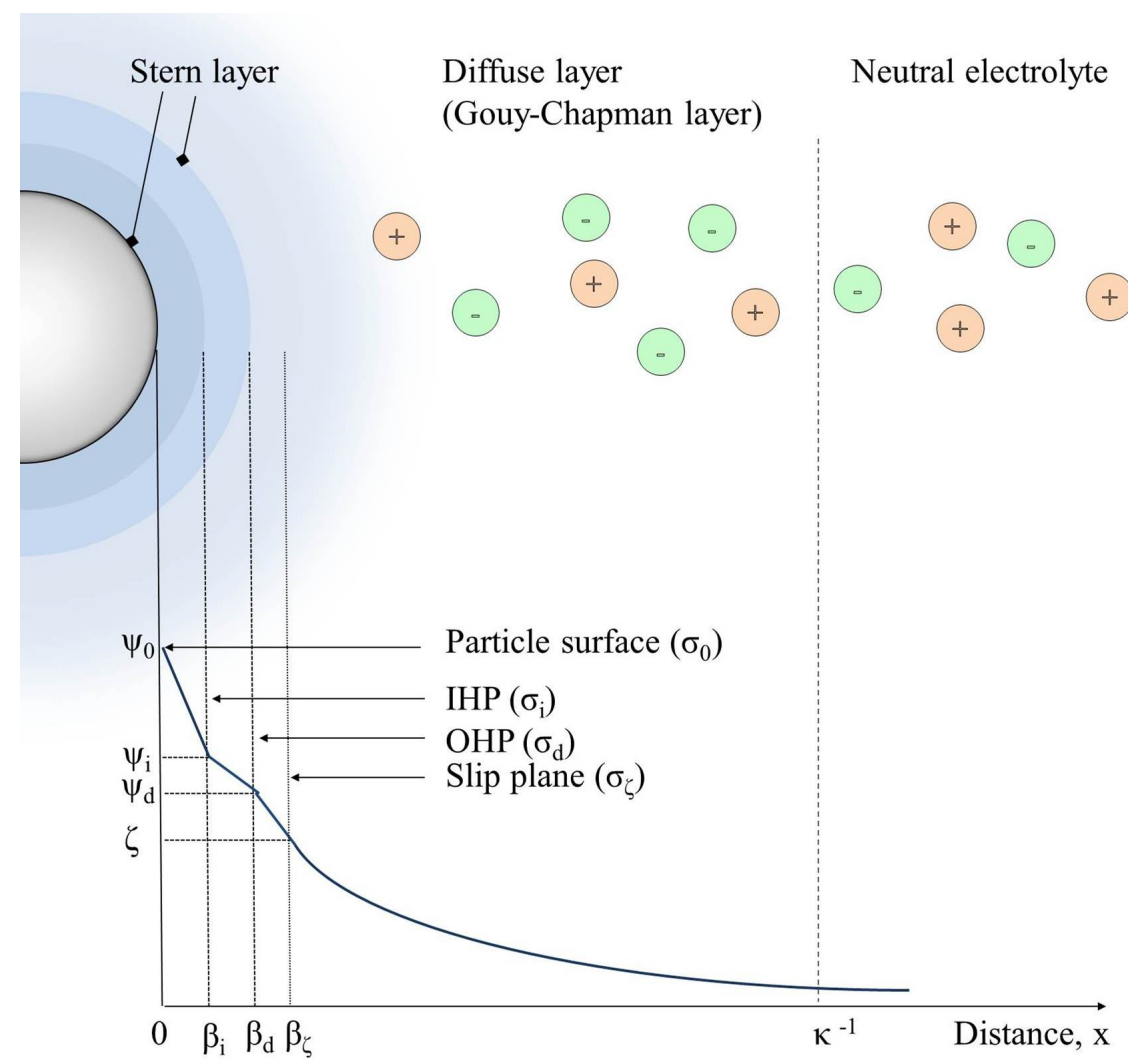

Figure 4.1: Schematic representation of the charges and potentials existing at a positively charged particle. The area found between the solid surface (with potential $\psi_{0}$ and charge density $\sigma_{0}$ ) and inner Helmholtz plane (at a distance $\beta_{i}$ from the solid surface) is uncharged. The IHP (with potential $\psi_{i}$ and surface charge $\sigma_{i}$ ) is the location where ions are specifically adsorbed. The diffuse part of the double layer begins at $\mathrm{x}=\beta_{d}$ (at the outer Helmholtz plane with potential $\psi_{d}$ and surface charge $\sigma_{d}$ ). At a distance $\mathrm{x}$ $=\beta_{\zeta}$, the slip plane or shear plane is situated and the potential found there is the electrokinetic or zeta potential, $\zeta$ and the electrokinetic charge density is $\sigma_{\zeta} \cdot \kappa^{-1}$ is the Debye length and also a measure of thickness of the diffuse layer of the EDL. 


\subsection{Electrokinetic phenomena. Zeta Potential}

Direct access to EDL quantities as surface charge density or potential is in most cases (with the notable exception of the mercury/solution interface, of scarce applied interest) impossible. Fortunately, since the pioneering works of Helmholtz or von Smoluchowski (see (Lyklema, 1991; Delgado \& Arroyo, 2002; Delgado et al., 2007)), it was demonstrated that it is factible to reach indirectly such information by applying an external field (electric, pressure gradient, gravitational, acoustic) to the disperse system and analyse experimentally its response. The information is indirect in the sense that it requires a theoretical model relating some measured quantity to the EDl quantities.

To begin with, it is admitted that generally an immobile, extremely thin layer of liquid adheres to the solid surface. Such layers known as hydrodynamically stagnant layer, and extends to the certain distance $\left(\beta_{\zeta}\right)$, where the so-called hydrodynamic slip plane is located. It is generally assumed that the slip plane is situated in the close vicinity of the outer Helmholtz plane. In this layer the main assumption is that no liquid motion is allowed although some models admit that ion migration by diffusion or electric transport may be possible, leading to the existence of a stagnant-layer conductivity or SLC, not considered in the present work. Out of the stagnant layer the action of external fields may set liquid into motion, and in general the phenomena associated to the subsequent relative motion between the liquid and the solid is known generally as electrokinetic phenomena. Because the motion occurs at the position $\beta_{\zeta}$, the potential (or charge) involved is not the surface potential but a different (typically smaller) one, known as electrokinetic or zeta potential $(\zeta)$. Even so, it is possible to find rigorous theoretical models capable of yielding information about $\zeta$ or its corresponding surface charge density, $\sigma_{\zeta}$.

The whole of electrokinetic phenomena come from two universal effects: convective electric surface current and electro-osmosis flow inside the electric double layer. For nonconducting solids, Smoluchowski has obtained equations for two general electrokinetic effects. They permit for extending the theory for all electrokinetic phenomena. The Smoluchowski's hypothesis is acceptable for every particle shape or pores within the solid, given that the (local) degree of curvature of radius $a$ is much greater than the Debye length $\kappa^{-1}$,

$$
\kappa a \gg 1,
$$


and $\kappa$ is given by

$$
\kappa=\left\{\frac{\sum_{i=1}^{N} e^{2} z_{i}^{2} n_{i}}{\varepsilon_{r s} \varepsilon_{0} k T}\right\}^{1 / 2},
$$

where $e$ is the elementary charge, $z_{i}$ is the charge number and $n_{i}$ represents number concentration of ion $i$ (the solution is composed of $N$ ionic species). Moreover, $\varepsilon_{r s}$ represents the relative permitivity of the solution and $\varepsilon_{0}$ the electric permitivity of vacuum. $k$ in this equation is the Boltzmann constant and $T$ is the temperature. Numerous aqueous solutions fulfil this condition, except those dispersions with particles little in size being in media of low ionic strength.

Electrophoresis is the movement of a colloidal particle with regards to the solution subjected to an external field, $\mathrm{E}_{\infty}$, which is invariable in time and not conditioned by location. This electrokinetic effect is reciprocal of electro-osmosis. The mobility value is positive if colloidal particles transfer in the direction of lower potential (negative electrode) and in reverse in the situation of negative mobility value. The velocity of the particle, $\mathbf{v}_{e}$, in relation to a solution at rest is given by:

$$
\mathbf{v}_{e}=\frac{\varepsilon_{r s} \varepsilon_{0} \zeta}{\eta} \mathbf{E} .
$$

The last expression is the Helmholtz-Smoluchowski equation for electrophoresis. For constant and not very high electric fields, a linear relation between electrophoretic velocity, $\mathbf{v}_{e}$ and the externally applied field, $\mathbf{E}$ can be found:

$$
\mathbf{v}_{e}=u_{e} \mathbf{E},
$$

and $u_{e}$ represents the electrophoretic mobility given by:

$$
u_{e}=\frac{\varepsilon_{r s} \varepsilon_{0} \zeta}{\eta} .
$$

Electrophoresis will be used in the present work, but mentions should be made to other electrokinetic phenomena, equally important from both fundamental and applied points of view:

- Electro-osmosis is the movement of the liquid adjoining to a solid (it may be also a porous plug, a capillary or a membrane) which is charged due to externally applied electric field. 
- Sedimentation potential is a potential gradient, $V_{s}$ detected by two electrodes located at a fixed vertical distance in the suspension of particles, exposed to a gravitational field g. The sedimentation (or, for that matter, flotation or centrifugation) potential is generated when the gravitational or centrifugal field sets the charged particles in motion.

- Streaming potential and streaming current are electrokinetic phenomena in which the movement of the solution is imposed by an applied pressure difference. The movement of this charged solution brings about an electric current (streaming current, $I_{s t r}(\mathrm{~A})$ ) when there exists a return track for the charges, or, in other case, an electrical potential (streaming potential, $U_{\text {str }}(\mathrm{V})$ ) when the electrodes are just connected to a high-input impedance voltmeter.

- Electrorotation is the rotational movement of colloidal particles which is caused by an applied rotating AC field of certain frequency. The torque required to promote the rotation is due to the phase lag between the field-induced electric dipole of the particle and the field itself.

- Dielectrophoresis occurs when particles are subjected to a harmonic AC field, with some specified spatial variation. The phenomenon of dielectrophoresis consists of the translational motion of the particles towards or away of the high-field area, depending again on the characteristics of the induced dipole moment.

- Diffusiophoresis takes place when the colloidal particles are in motion as a result of an externally applied electrolyte concentration gradient (or, more generally, in a gradient of chemical composition of the solvent, as the motion may also appear in nonaqueous suspensions with non-homogeneous solvent composition.

- Low-Frequency Dielectric Dispersion, LFDD. This is the name given to the frequency dependence of the dielectric permittivity of a suspension, in the radiofrequency range. It is manifestation of the existence of certain characteristic relaxation times for the various polarization mechanisms of the EDL. It happens to be a technique very sensitive to the electrochemistry of the EDLs of the particles, although it is not free of experimental difficulties (Grosse \& Delagdo, 2010).

- Electroacustic phenomena. Related to the previous one, but typically applicable in the 1-20 MHz frequency region only. The techniques most widely used are ESA(electrokinetic sonic amplitude), that is, the generation of sound waves through application of an alternating electric field to the suspension, and CVP or CVI (colloid vibration 
potential or current), where the passage of a sound wave produces an alternating electric field. From both, information about the dynamic electrophoretic mobility (the AC counterpart of classical electrophoretic mobility) can be obtained, yielding information about the $\zeta$ potential and the size distribution of a (often concentrated) suspension of particles.

\subsection{Methodology}

Electrophoretic mobility measurements were carried out in a Zetasizer NanoZS (from Malvern Instruments, U.K.). In the case of suspensions with various $\mathrm{pH}$ values they were prepared by simply adding dropwise a small amount of the iron oxide mother suspensions to $50 \mathrm{~mL}$ of $5 \mathrm{mM} \mathrm{KNO}_{3}$ (fixed ionic strength) until finally obtaining a slightly turbid solution adequate for this type of determination. The $\mathrm{pH}$ value of the suspensions was then adjusted by adding a suitable amount of $\mathrm{KOH}(0.01$ or $0.1 \mathrm{M})$ or $\mathrm{HNO}_{3}(0.01$ or $0.1 \mathrm{M})$. The suspensions were left unperturbed at least $3 \mathrm{~h}$, and then the electrophoretic mobility was determined. For each suspension, 3 measuring runs were taken, with 3 cycles in each run. The temperature was $25.0 \pm 0.5^{\circ} \mathrm{C}$.

In the case of suspensions with different ionic strengths, these were prepared by suspending a small amount of the iron oxide mother suspensions in $\mathrm{KNO}_{3}$ solutions containing the specified amount of electrolyte, in the concentration range $10^{-4} \mathrm{M}$ to $0.1 \mathrm{M}$. After preparation, suspensions were equilibrated for $3 \mathrm{~h}$ as before. When necessary, the zeta potential was obtained from mobility data using the O'Brien and White theory (O'Brien \& White, 1978), and the maximum relative uncertainty was typically below 5 $\%$.

\subsection{Results and discussion}

\subsubsection{Effects of $\mathrm{pH}$ and ionic strength on the electrokinetic properties of the maghemite nanocomposites. Design I.}

As mentioned earlier, two designs of nanoparticulate vehicles have been investigated in this work for doxorubicin transport. The synthesis of magnetic nanoparticles, functioning as a magnetic core, and the process of their 
silica coating are two common steps in fabrication of both types of vehicles. As these steps are fundamental in the overall vehicle formation, there is a need for control of their stability. This can be tested, and to some extent predicted, by means of electrophoresis, which may be considered as a very useful tool in the investigation of the effectiveness of the surface modification employed in the situations referred to above. Another argument proving the significance of surface charge evaluation regards the biodistribution of nanocompounds in function of this quantity $\left(u_{e}\right)$ measurements (Thode et al., 2000).

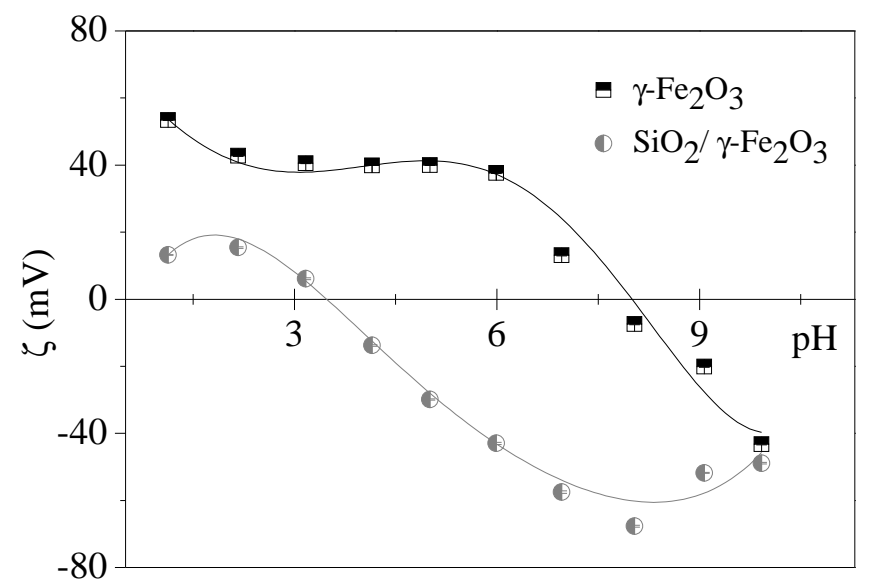

Figure 4.2: Zeta potential of $\gamma-\mathrm{Fe}_{2} \mathrm{O}_{3}$ and $\mathrm{SiO}_{2}$-coated $\gamma-\mathrm{Fe}_{2} \mathrm{O}_{3}$ suspensions as a function of $\mathrm{pH}$.

Zeta potential titration permitted to confirm the $\mathrm{pH}$ value of about 7.5 for the isoelectric point of maghemite (Fig. 4.2), in accordance with prior results showing that ferrofluids are generally stable only in highly acidic or basic pHs (Halbreich et al., 1997). The existence of the silica layer changes the $\zeta \mathrm{pH}$ tendency in an important manner, in such a way that the composite particles behave similarly to pure silica. Note that the isoelectric point of the silica-coated maghemite nanoparticles is approximately 3.5 , very distant from that of maghemite and actually closer to that of silica (Rudzka et al., 2012). The $\gamma-\mathrm{Fe}_{2} \mathrm{O}_{3}$ potential instability at the beginning at natural $\mathrm{pH}$, which is connected with its low surface potential and small particle size, will be very likely modified through the existence of the outer silica shell. It is worth noting that silica-coated maghemite nanocomposites may form stable suspensions under the $\mathrm{pH}$ conditions characteristic for biological fluids and may be characterized by a surface chemistry easy 
to be predicted because of the well-known reactivity of silica with respect to coupling agents. The silica shell onto maghemite nanoparticles causes an increase in their chemical stability, thus affecting their performance for bioapplications, particularly when employed for photodynamic therapy purposes. The outer silica shell also enhances the polyelectrolyte compatibility. As mentioned, generally the magnetic core contains more than one iron oxide nanoparticle, due to the aggregation of iron oxide nanoparticles before or during the coating procedure.

In the case of the first type of synthesized DOX vehicles the molecular self-assembly (layer by layer, or $L b L$ ) method was the first phase in a two-step process for the gold layer creation. Every consecutive precursor polyelectrolyte film (cationic PDADMAC was first adsorbed, anionic PSS deposition was coming after) showed a very important impact on the surface charge of the silica-coated maghemite nanoparticles and their subsequent modifications. Figure 7.7(a) displays the radical changes of the electrophoretic mobility of silica-coated magnetic nanoparticles goes through after the polyelectrolyte deposition. It is noteworthy that through deposition of one polyelectrolyte layer, there is the possibility of absolute control of the surface charge, in such a way that the surface can be changed from highly positive (for the PDADMAC deposition) to very negative (for the PSS deposition). Subsequently, data pertaining to the third layer (PDADMAC on top of PSS) are practically the same as those achieved after the first PDADMAC adsorption, and therefore they will not be presented. These three deposits of polyelectrolytes will produce uniform nanocompounds with positive charge excellently prepared for the negative gold deposit (Salgueiriño Maceira et al., 2006). It is once more verified that the LbL method is an outstanding tool for taking advantages of the electrostatic attraction between charged particles and layers to be adsorbed.

Fig. 7.7(b) presents the trend of variation of the electrophoretic mobility with ionic strength. It is worth noting the significant effect of the polyelectrolyte deposits on the mobility of maghemite nanoparticles with silica coating, for the entire range of ionic strengths examined. Interestingly enough, the results published by Ohshima regarding the finite value of the electrophoretic mobility at very high ionic strengths for soft particles (with polyelectrolyte coating) is validated in our case (Ohshima, 2002). Electrophoretic mobility evaluation proves as well to be an excellent probe for testing the adsorption of small gold seeds onto the PDADMAC-coated silica-maghemite compounds. Results in Fig. 7.6 show that after three steps of gold layer formation, the electrophoretic mobility of these nanocom- 


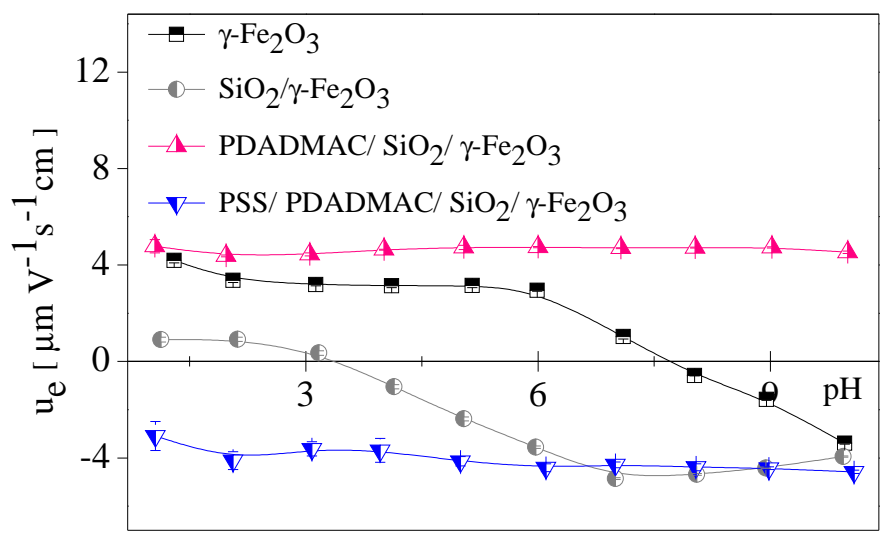

(a) $u_{e}$ as a function of $\mathrm{pH}$ in $5 \mathrm{mM} \mathrm{KNO}_{3}$.

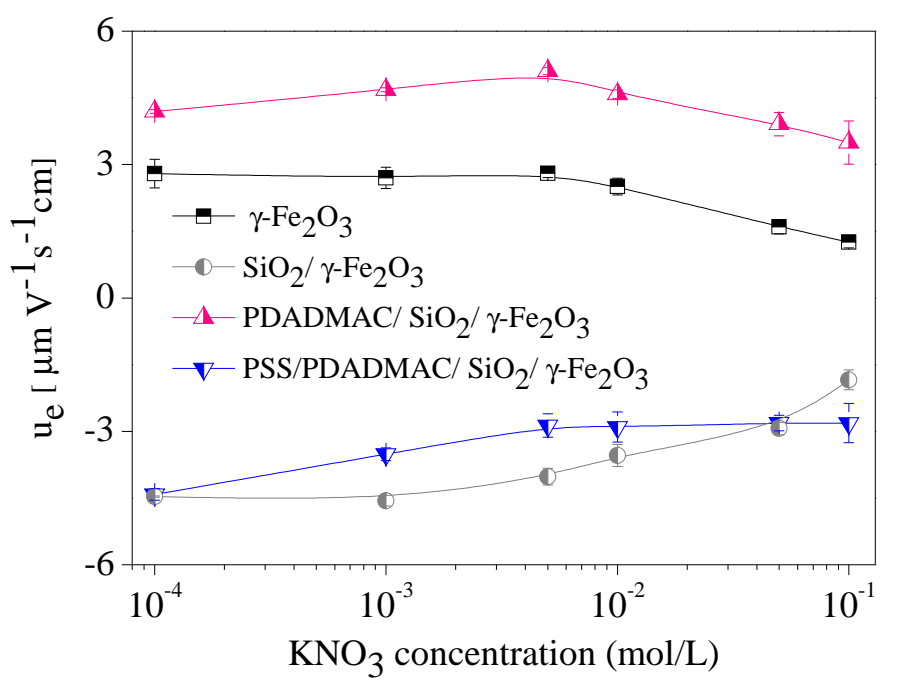

(b) $u_{e}$ as a function of ionic strength at natural $\mathrm{pH}$.

Figure 4.3: The electrophoretic mobility of maghemite $\left(\gamma-\mathrm{Fe}_{2} \mathrm{O}_{3}\right)$, and maghemite/silica $\left(\mathrm{SiO}_{2} / \gamma-\mathrm{Fe}_{2} \mathrm{O}_{3}\right)$ before and after adsorption of PDADMAC and PSS/PDADMAC polyelectrolyte layers for the first type of designed DOX vehicles. 


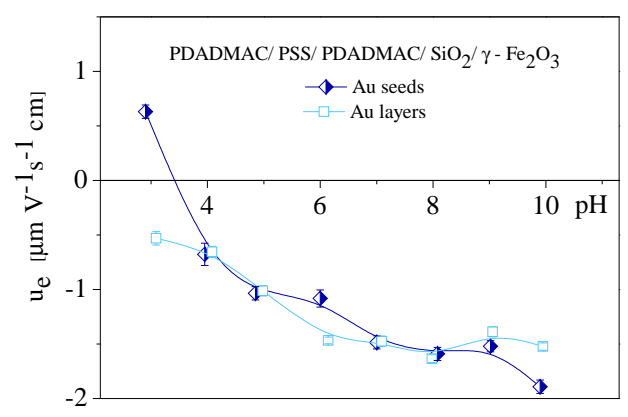

Figure 4.4: Comparison of the $\mathrm{pH}$ effect on the electrophoretic mobility of silicacoated maghemite nanoparticles with deposited gold seeds and on that of final nanostructures with three gold layers, for the first type of synthesized vehicles.

pounds is almost impossible to differentiate from that of the nanoparticles with the gold seeds deposited, this being a good manifestation of the effectiveness of the gold layer creation.

\subsubsection{Results for Design II}

The second DOX vehicle design is quite different. To begin with, as above described, their preparation process as for the gold layer formation consists only in one step, namely adsorption of APTMS (short-tailed amine functionalised silane) onto the surface of magnetic silica spheres. APTMS plays the role of amine functional groups precursor. Fig. 7.8(a) displays the electrophoretic mobility of the bare $\gamma-\mathrm{Fe}_{2} \mathrm{O}_{3}$ nanoparticles, the $\mathrm{SiO}_{2} / \gamma-\mathrm{Fe}_{2} \mathrm{O}_{3}$ nanoparticles and APTMS-treated magnetic silica spheres as a function of $\mathrm{pH}$. It is obvious that the cationic polyelectrolyte APTMS may be employed to functionalize the magnetic silica spheres, making them positive in a broad $\mathrm{pH}$ range, until a $\mathrm{pH}_{i e p} \approx 7$. The significant detail is that APTMS modification causes that the nanocompounds are already prepared to function as a seat for small gold nanoparticles, as needed (Fig. 7.9). The change of the isoelectric point is an indication of the gold seeds adsorption. In both graphs (Fig. 7.8(b) and Fig. 7.9) we observe that the positive mobility of APTMS-covered particles rises in the $\mathrm{pH}$ range 1 - 3: two possible explanations can be proposed to this fact. One is that the rise of ionic strength from $\mathrm{pH} 3$ to 1 will produce compression of the extended APTMS chains, shifting the electrokinetic plane towards the surface and reducing the zeta potential. The second possibility, probably occurring as well, is that the increase in the negative surface charge of the exposed silica, and also of gold, in the $\mathrm{pH}$ range indicated will favor adsorption of the protonated APTMS molecules. The electrophoretic mobility evolution is compatible with the formation of the gold layer in the latest phase of the nanocompounds creation: observe in Fig. 7.9 how the mobility shifts to more negative values 


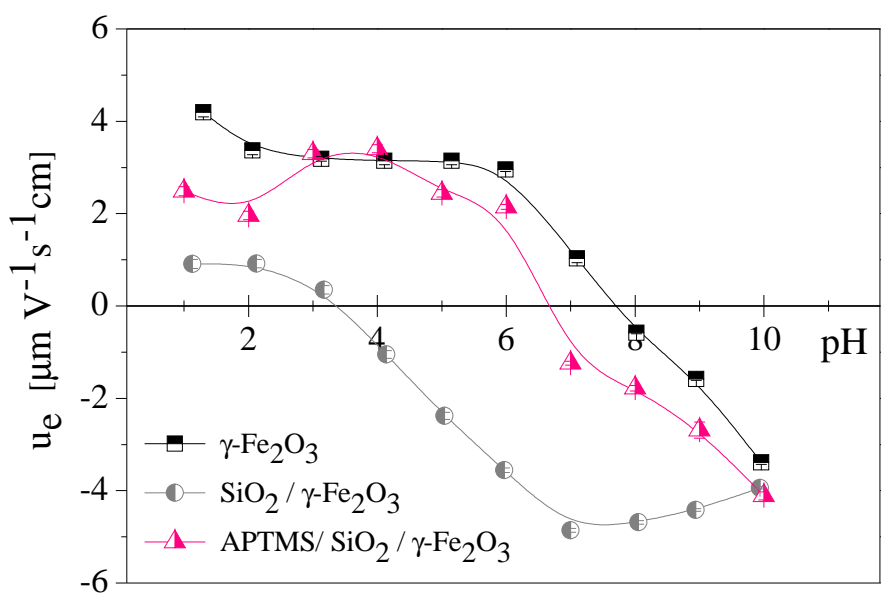

(a) $u_{e}$ as a function of $\mathrm{pH}$.

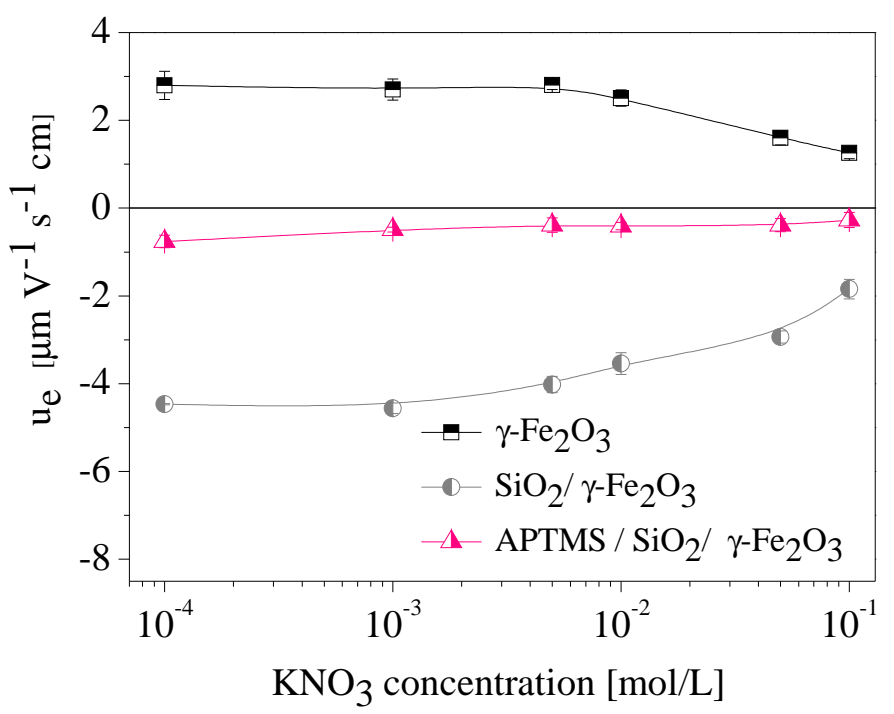

(b) $u_{e}$ as a function of ionic strength.

Figure 4.5: Electrophoretic mobility of bare $\gamma-\mathrm{Fe}_{2} \mathrm{O}_{3}$, silica-coated maghemite, and silica/maghemite nanocompounds with an APTMS layer deposited. 


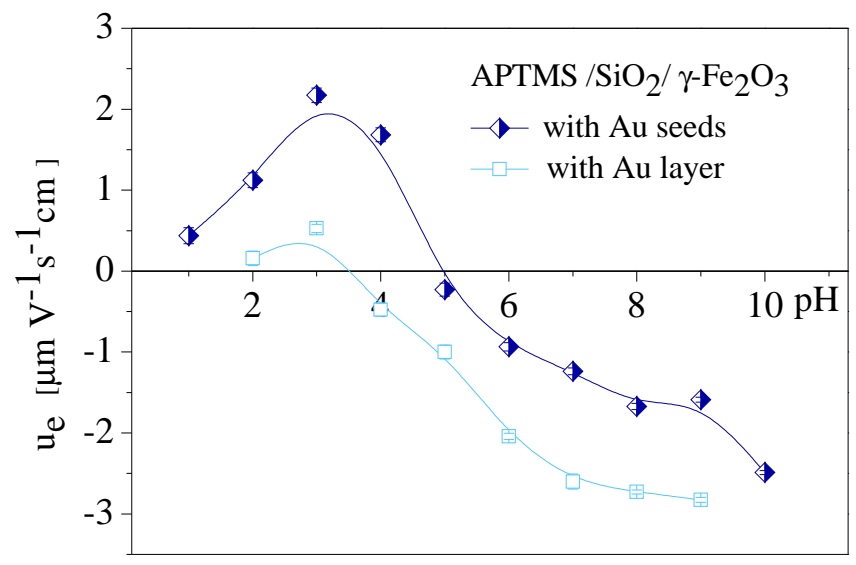

Figure 4.6: Mobility behavior after coating with Au seeds and gold layer for the second type of DOX vehicles.

when compared to that determined when only the gold seeds are deposited on the nanocomposites surface. 



\section{Chapter 5}

\section{Magnetic characteristics}

Because the key aspect of the particles described is the fact that they possess a magnetic core, it seems convenient to provide the magnetic characterization of the vehicles designed, and their consequences. These are the aims of the present chapter. 



\subsection{Generalities}

We can say that all materials present magnetic properties to some extent, and they will differ, for a given material, on the sample geometry, temperature, particle size, etc., and from one material to another in function of the magnetic moments of their atoms and possible interactions between them. In practical terms they can be categorized with the use of the magnetic susceptibility $\chi$ (dimensionless), relating magnetization $\mathbf{M}$ and field strength $\mathbf{H}(\mathbf{M}=\chi \mathbf{H}$, and the magnetic susceptibility will be a scalar quantity for an isotropic material, as assumed here). The experimentally accessible quantity is the mass susceptibility, $\chi_{m}$, relating the mass magnetization (magnetic moment per unit mass) to the applied field. If the density of the material $\rho_{m}$ is known, the relationship between them is immediately given by $\chi=\chi \rho$. Nevertheless, it sometimes happens that the particles are constituted by several materials, and their density is not easy to evaluate. In that case, the mass susceptibility is preferred ${ }^{1}$. Another observation that might be in order concerns the system of units typically used in experimental magnetism (and in other fields, in fact: for practical reasons, the cgs units are still frequently used). All magnetometers provide the magnetization in emu/g (mass magnetization in cgs units) and the field in Oersted (instead of $\mathrm{A} / \mathrm{m}$ ). Care must be taken in changing units if necessary:

$$
\begin{array}{r}
1 \mathbf{A} / \mathbf{m}=4 p i \times 10^{-3} \mathbf{O e} \\
1 \mathbf{T}=10^{4} \mathbf{G} \\
\chi^{\mathbf{S I}}=4 \pi \times \chi^{\mathbf{c g s}} \\
\chi_{m}^{\mathbf{S I}}\left(\mathbf{m}^{\mathbf{3}} / \mathbf{k g}\right)=\chi_{m}^{\mathbf{c g s}}\left(\mathbf{c m}^{\mathbf{3}} / \mathbf{g}\right)
\end{array}
$$

\subsubsection{Ferromagnetism and ferrimagnetism}

As it is well known, some metals and alloys are characterized by their atoms possessing permanent magnetic moments, capable of interacting with their neighbours by exchange coupling, and as a consequence favouring parallel

\footnotetext{
${ }^{1}$ Note that the SI units of $\chi_{m}$ are $\mathrm{m}^{3} / \mathrm{kg}$.
} 
(or antiparallel) orientations (in the absence of applied field) extending to the whole sample or to some region of it (see details below). When the exchange interaction favours parallel coordination, we speak of ferromagnetic materials. Typical examples include CO, Ni, Gd or $\alpha$-iron. They are characterized by large initial (low-field), positive susceptibilities (they can be as high as $10^{6}$ ), so that the dependence of flux density $\mathbf{B}$ and field strength can be approximately given by $\mathbf{B}=\mu_{0}(\mathbf{H}+\mathbf{M}) \cong \mu_{0} \mathbf{M}$.

Depending on the characteristics of the atoms, the exchange coupling can lead to antiparallel orientation of neighbour spins, characterizing the antiferromagnetic behaviour. As a significant example of this kind of behaviour, we mention manganese oxide, $\mathrm{MnO}_{2}$ : whereas $\mathrm{O}^{2-}$ ions do not present a net magnetic moment due to a complete cancellation of orbital and spin magnetic moments, $\mathrm{Mn}^{2+}$ ions, with a spin moment, are arranged in the crystal structure in such a way that the moments of adjoining ions are in antiparallel orientation. Clearly, the resultant magnetic moments will cancel each other and it will result in no magnetic moment in the material.

For our purposes, we must still consider another kind of magnetic behaviour, shown by some ceramic materials (known as ferrites), notably magnetite and maghemite. They may be represented by the chemical formula $\mathrm{MFe}_{3} \mathrm{O}_{4}$, where $\mathrm{M}$ symbolizes some metal. The standard ferrite material is magnetite $\left(\mathrm{Fe}_{3} \mathrm{O}_{4}\right)$, or $\mathrm{Fe}^{2+} \mathrm{O}^{2-}-\left(\mathrm{Fe}^{3+}\right)_{2}\left(\mathrm{O}^{2-}\right)_{3}$, with a crystal structure of inverse spinel, which is essential in explaining the magnetization of these ferrites. The divalent iron ions are located in tetrahedrally coordinated positions, whereas the trivalent irons are equally located in octahedral and tetrahedral ones, as follows:

$$
8\left\{\mathrm{Fe}^{3+}\right\}_{\mathrm{T}} 8\left\{\mathrm{Fe}^{3+}, \mathrm{M}^{2+}\right\}_{\mathrm{O}} 32 \mathrm{O}
$$

Each divalent iron contributes 4 Bohr magnetons $\left(4 \mu_{B}\right)$ to the total magnetic moment of the crystal, and each $\mathrm{Fe}^{3+}$ gives $5 \mu_{B}$. In reality, the trivalent cations undergo antiparallel exchange interactions between the octahedral and tetrahedral irons, so that each magnetite molecule contributes 4 Bohr magnetons. In the case of maghemite, the crystal structure is also inverse spinel, but it is defective in $\mathrm{Fe}^{2+}$, with a distribution of $\mathrm{Fe}^{3+}$ as indicated below:

$$
8\left\{\mathrm{Fe}^{3+}\right\}_{\mathrm{T}} 8\left\{\mathrm{Fe}_{5 / 3}^{3+},()_{1 / 3}\right\}_{\mathrm{O}} 32 \mathrm{O}
$$

corresponding to $2.5 \mu_{B}$ per formula unit. The cancellation of $\mathrm{T}$ and $\mathrm{O}$ sites leads to a a ferrimagnetic behaviour, similar to magnetite. 


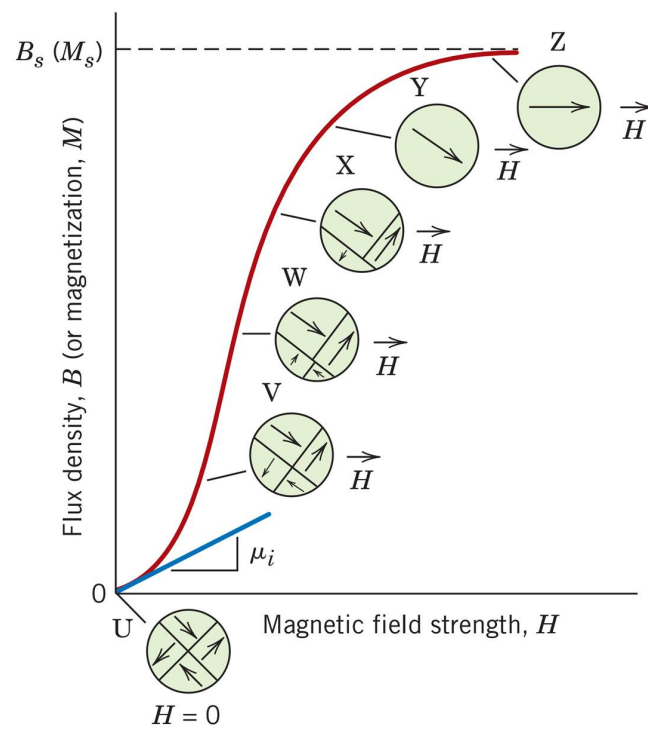

Figure 5.1: Magnetic flux density as a function of magnetic field strength for ferromagnetic and ferrimagnetic materials. As indicates the curve, at the beginning the sample was unmagnetized. Arrangements of domains during various phases of magnetization are depicted. The values of $\mathrm{B}_{s}, \mathrm{M}_{s}$ and initial permeability $\mu_{i}$ are as well pointed out (Callister, 2005).

It may be recalled that, for sufficiently large field strengths the magnetization of ferro- or ferrimagnetic materials ceases to be proportional to $\mathbf{H}$ (and so does the induction B), as shown in Fig. 5.1. The magnetic permeability is maximum at low fields $\left(\mu_{i}\right)$, and decreases as the field raises, so that, at sufficiently large fields the magnetization does not increase further and saturation magnetization is said to be reached. The mechanism, based on the growth of magnetic domains (regions of equal moment orientation) whose orientation is close to that of the field direction at the expense of domains in other directions, is illustrated in Fig. 5.1. This process goes on as the magnetic field strength is raised until the macroscopic sample finally turns into a single domain that is almost oriented in the direction of the applied magnetic field. This is the saturation magnetization (point $\mathrm{Z}$ in Fig. $5.1)$.

There is still another aspect of the magnetic behaviour of ferromagnets that should be reminded at this point. This is the phenomenon of hysteresis, by virtue of which the magnetization does not follow its original track when the field is decreased back from a given value (from saturation, for instance), as shown in Fig. 5.2. When this happens, the magnetization or induction at zero field do not come back to zero (remanence, $B_{r}$ in the Figure), and a coercive field (or coercive force) $H_{c}$ is needed to cancel the magnetization.

Fig. 5.2 depicts a hysteresis loop which reaches saturation magnetiza- 
Figure 5.2: The flux density (B) as a function the field strength $(\mathbf{H})$ for material which exhibits ferromagnetic behaviour and is exposed to forward and reverse saturations (point $\mathrm{S}$ and $\mathrm{S}^{\prime}$ ). The red line symbolizes the hysteresis loop and the blue one represents the early magnetization state. The magnitudes of remanence $\mathrm{B}_{r}$ and the coercive force $\mathrm{H}_{c}$ are also pointed out (Callister, 2005).

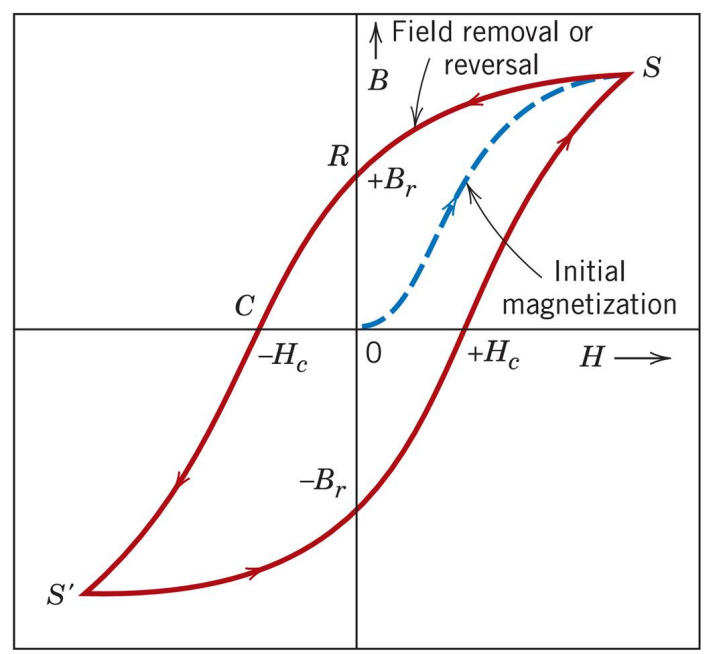

tion. Certainly, there is no need to rise the magnetic field up to saturation before reversing its direction. Fig. 5.3 presents a loop (NP loop) that represents a hysteresis which does not reach saturation. Moreover, reversion of the field orientation at any stage of the curve will produce different hysteresis loops (this is the case of the LM loop in Fig. 5.3).

At this point is may be helpful to contrast the $B$-versus- $H$ behaviours of paramagnetic, diamagnetic, and ferromagnetic/ferrimagnetic substances. A comparative relation like this is depicted in Fig. 5.4. It helps in giving arguments for calling paramagnetic and diamagnetic materials "nonmagnetic ". It suffices for this to compare the $B$ values from two plots at $H$ values of $50 \mathrm{~A} / \mathrm{m}$. In case of ferromagnetic/ferrimagnetic substances the flux density $B$ reaches $1.5 \mathrm{~T}$, while for the paramagnetic/diamagnetic substances is approximately $5 \times 10^{-5} \mathrm{~T}$, either positive or negative.

As was mentioned before, the existence of hysteresis is one of the possible routes to hyperthermia: the energy needed for moving the domain walls in the process of magnetization is lost as heat as a consequence of the irreversibility of the magnetization curve. As a result, when we apply a time-varying magnetic field to a ferro- or ferrimagnetic material a continuous flow of energy will be produced into that material, which will hence increase its temperature. 


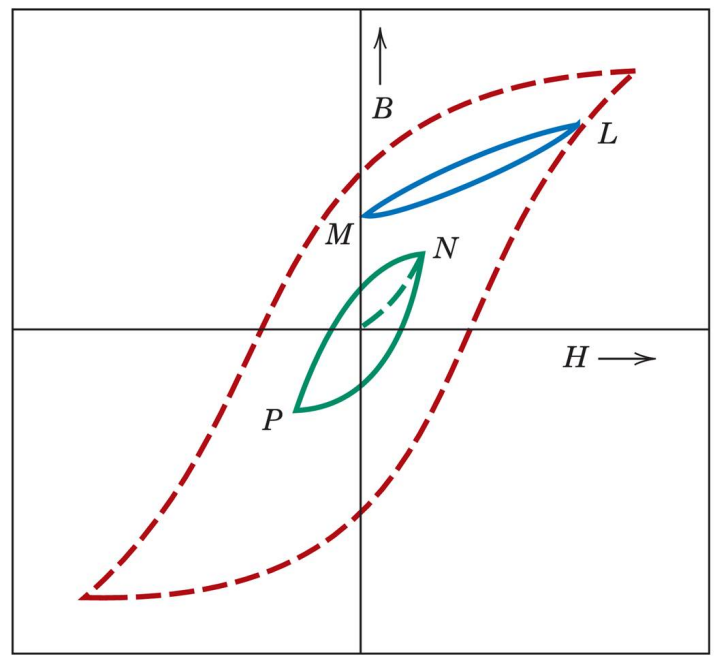

Figure 5.3: A hysteresis represents curve that does not reach saturation (NP curve) which is presented inside a hysteresis loop for ferromagnetic material (red dashed line). The $B-H$ dependence in case of field reversion without saturation is represented by the LM curve (Callister, 2005).

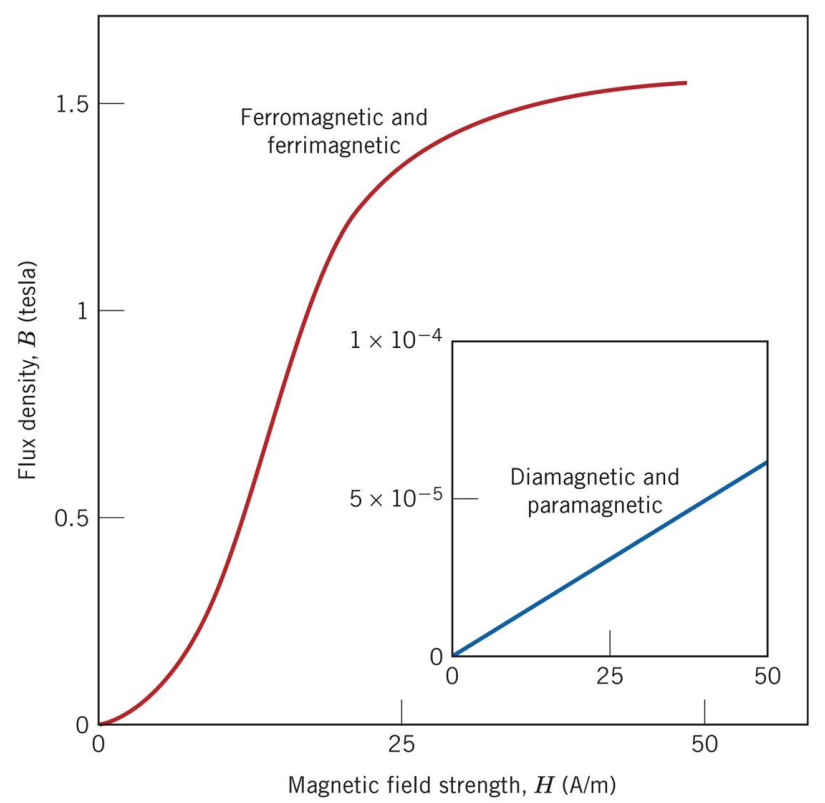

Figure 5.4: Plots of magnetic induction as a function of magnetic field strength for ferromagnetic/ferrimagnetic and diamagnetic/paramagnetic samples (inset). Note that the magnetic induction values generated in the latter cases are very small (Callister, 2005). 
A
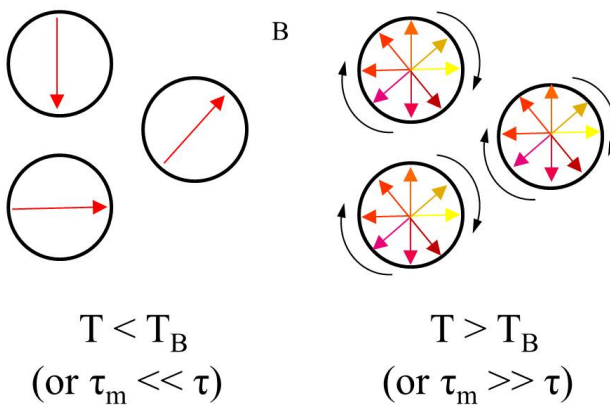

Figure 5.5: Illustration of the idea of superparamagnetism. The circles represent magnetic particles and the arrows inside them demonstrate the net magnetization direction. In A the temperature $(T)$ is much lower than the blocking temperature $\left(T_{B}\right)$ of the particles, or else the time between moment reversal ( $\tau$-relaxation time) is much longer than the measurement time $\left(\tau_{m}\right)$ so that the net moments are quasi-static. In B the temperature is higher than $T_{B}$, or relaxation time is much shorter than $\tau_{m}$, meaning that without external field applied the time-averaged net moment on the particles is equal to zero.

\subsubsection{Superparamagnetism}

The characteristics of the hysteresis cycle are related to the pinning force experienced by the walls of the magnetic domains when they meet impurities or grain boundaries, but also to the magnetic anisotropy occurring inside the crystal lattice of the material. Additionally, the shape of the curves is partially defined by the particle size: i) micron-sized particles (multi-domain ground state), result in a thin hysteresis loop, due to the fact that the walls of the domains require low energy to move; ii) smaller sized particles (single-domain ground state) present a broad hysteresis loop; iii) nano-sized particles (tens of nanometers) present a phenomenon known as superparamagnetism. The whole particle can be considered as a single magnetic domain, and its corresponding $M-H$ dependence is anhysteretic and sigmoidal (Fig. 5.6).

This is the kind of magnetic behaviour normally exhibited by magnetic nanoparticles, for which thermal energy can be sufficient to spontaneously change the magnetization inside each magnetic nanoparticle. As a result, in the absence of an external magnetic field (Fig. 5.5), the net magnetic moment of the system containing the nanoparticles will tend to zero. However, when an external magnetic field is applied, a net average alignment of the magnetic moments will be observed. Something similar happens in case 
of paramagnetic materials, with the difference that the individual magnetic moment now is that of the magnetic nanoparticle (containing hundreds or thousands of atoms), which can hence be up to $10^{4}$ times larger than that of an individual atom. This feature (characterized by no remaining magnetization in absence of magnetic field) allows the magnetic nanoparticles to keep the colloidal stability and prevent agglomeration, which is essential for biomedical applications. From what has been said, the superparamagnetism of the particle is based on the physical law of activation for the relaxation time $\tau$ of the net magnetization:

$$
\tau=\tau_{0} \exp \left(\frac{\Delta E}{k_{B} T}\right)
$$

where $\Delta E$ expresses the energy barrier to moment reversal, and $\mathrm{k}_{B} \mathrm{~T}$ represents thermal energy. $\tau_{0}$ factor is slightly temperature-dependent when particles do not interact and take values of $10^{-10}-10^{-12} \mathrm{~s}$ (Brown, 1963). The origins of the energy barrier involves two effects: i) intrinsic (magnetocrystalline) and ii) extrinsic (anisotropies in shape). The activation energy $\Delta E$ is proportional to the particle volume $V$, and for this reason superparamagnetism (where thermally excited flipping of the direction of the net moment can take place) is only possible for small particles, for which $\Delta E \approx \mathrm{k}_{B} \mathrm{~T}$ at room temperature. Nevertheless, it is also very significant that superparamagnetism depends not only on temperature but also on the measurement time $\left(\tau_{m}\right)$ required by the employed technique (Fig. 5.5).

When $\tau \ll \tau_{m}$, the flipping is quick compared to the experimental time window and the particles seem to be paramagnetic, whereas if $\tau \gg \tau_{m}$ the flipping is slow and we can notice quasi-static properties (described as "blocked"state of the system). The definition given for a 'blocking temperature' $\left(\mathrm{T}_{B}\right)$ is the point between these two phases described above, which is characterized by the condition that $\tau=\tau_{m}$. The value of $\tau_{m}$ in case of a typical measurement can vary from slow and medium timescales (for diamagnetic materials $-10^{2} \mathrm{~s}$ and for AC susceptibility) to the fast timescales (for ${ }^{57} \mathrm{Fe}$ Mössbauer spectroscopy $-10^{-7}$ to $10^{-9} \mathrm{~s}$ ).

\subsubsection{Biomedical implications}

The phenomenology just described is of special interest for us in relation to the behavior of magnetic particles when injected in the body. In Fig. 5.6 a schematic plot illustrates a blood vessel with some magnetic colloids inside. Two types of injected particles coexist with biomolecules in the 
blood stream environment, and they have distinct responses to the applied magnetic field which can manifest in the shape of the magnetization curves.

The blood vessel itself exhibits: i) paramagnetic response (due to the Fe transported by the haemoglobin molecules); ii) diamagnetism (due to the proteins - composed only by $\mathrm{C}, \mathrm{H}, \mathrm{N}$ and $\mathrm{O}$ atoms). It is worth to remark that the magnetic signal obtained from haemoglobin is negligible compared to that of the particles injected in the blood stream, independently of the particle size. This intensified selectivity can be considered as one of the beneficial characteristics of biomedical applications of magnetic nanoparticles.

\subsection{The magnetic properties of maghemite and nanocomposites}

The magnetic study for this work was carried out using a Quantum Design MPMS XL Squid magnetometer from EVERCOOL (USA). For all samples the hysteresis cycle determination started from zero field up to $50 \mathrm{kOe}$, back to $-50 \mathrm{kOe}$ and again to $50 \mathrm{kOe}\left(1 \mathrm{Oe}=10^{3} / 4 \pi \mathrm{A} / \mathrm{m}\right)$. The plots of magnetization versus magnetic field ( $M-H$ loops) at room temperature for the bare superparamagnetic nanoparticles and for maghemite nanoparticles encapsulated into silica shells are displayed in Fig. 5.7. Our study shows that both bare and modified maghemite nanopartices present superparamagnetic behaviour at room temperature, suggesting that thermal fluctuations turn out to prevail over spontaneous magnetization at provided field (Sun et al., 2004), and the net magnetization when the external magnetic field is not applied is zero. The saturation magnetization reaches the values of $30.7 \mathrm{emu} / \mathrm{g}$ for bare maghemite nanoparticles at $50 \mathrm{kOe}$. This value is much lower than that of bulk maghemite, for which published data are about $74 \mathrm{emu} / \mathrm{g}$ (Berkowitz et al., 1968). Other values reported in the literature are $52 \mathrm{emu} / \mathrm{g}$ in case of particles with size of $15 \mathrm{~nm}$ and $31 \mathrm{emu} / \mathrm{g}$ for the ones of $7 \mathrm{~nm}$ (Millan et al., 2007). It should be recalled that the value of saturation magnetization for single-domain materials with superparamagnetic characteristics is dependent on particle size (Liu et al., 2000), because of the considerable effect of surfaces, where the exchange coupling is compromised ( $\mathrm{Yu} \&$ Chow, 2004): the surface spins of magnetic materials are deficient in absolute coordination and are consequently disordered. Therefore, they are less liable to be affected by modifications in the external field strength (Kachkachi et al., 2000; Kodama et al., 1996). This 

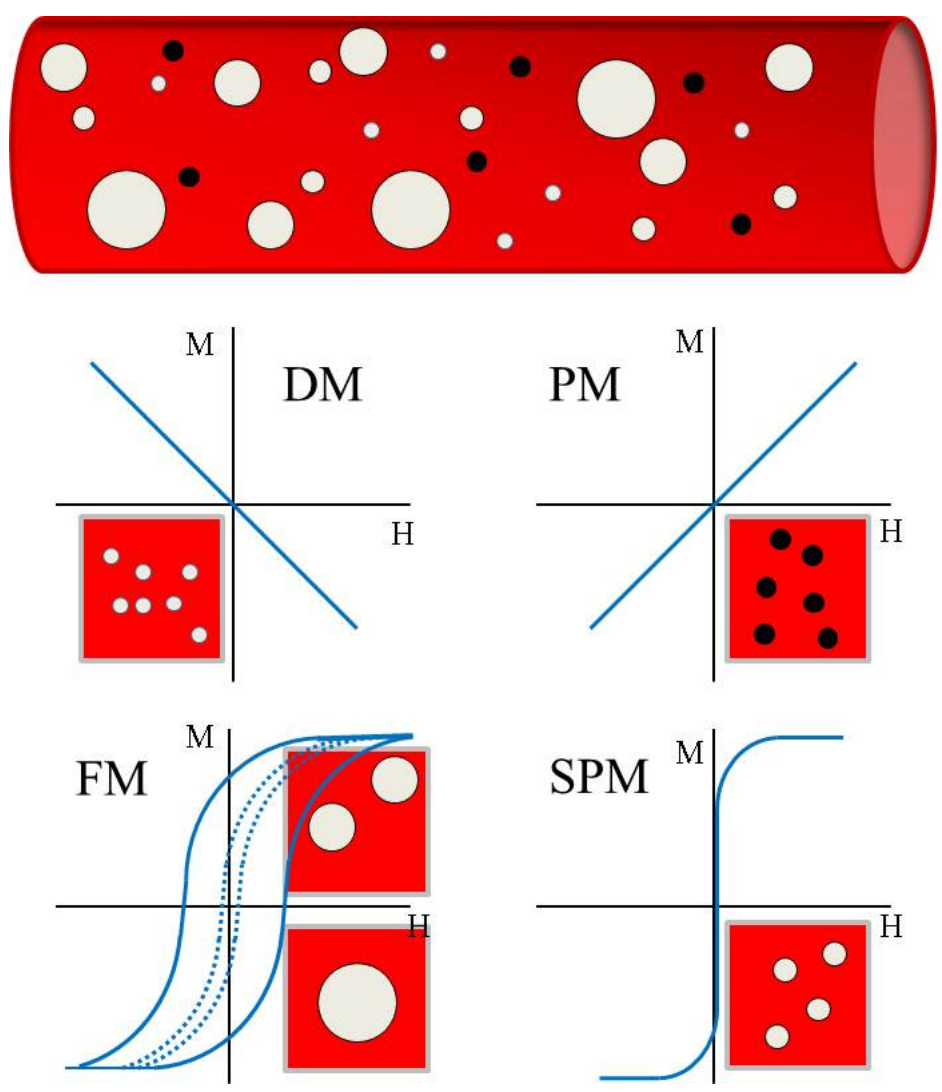

Figure 5.6: Magnetic responses characteristic for various types of magnetic materials: - This scheme presents a hypothetical situation in which particles with different sizes (from $\mathrm{nm}$ to $\mu \mathrm{m}$ scale) and with ferromagnetic properties have been injected intravenously. Four depicted M-H curves illustrate the behaviour of diamagnetic (DM) and paramagnetic (PM) biomolecules in the blood, and of the injected ferromagnetic particles (FM). In the last case there are three types of possible response: dots for multi-domain, and lines for single-domain or superparamagnetic (SPM) particles, in which response depends on the size of the particle. 
Figure 5.7: Magnetization curves obtained at roomtemperature for magnetic silica spheres and for bare maghemite nanoparticles.
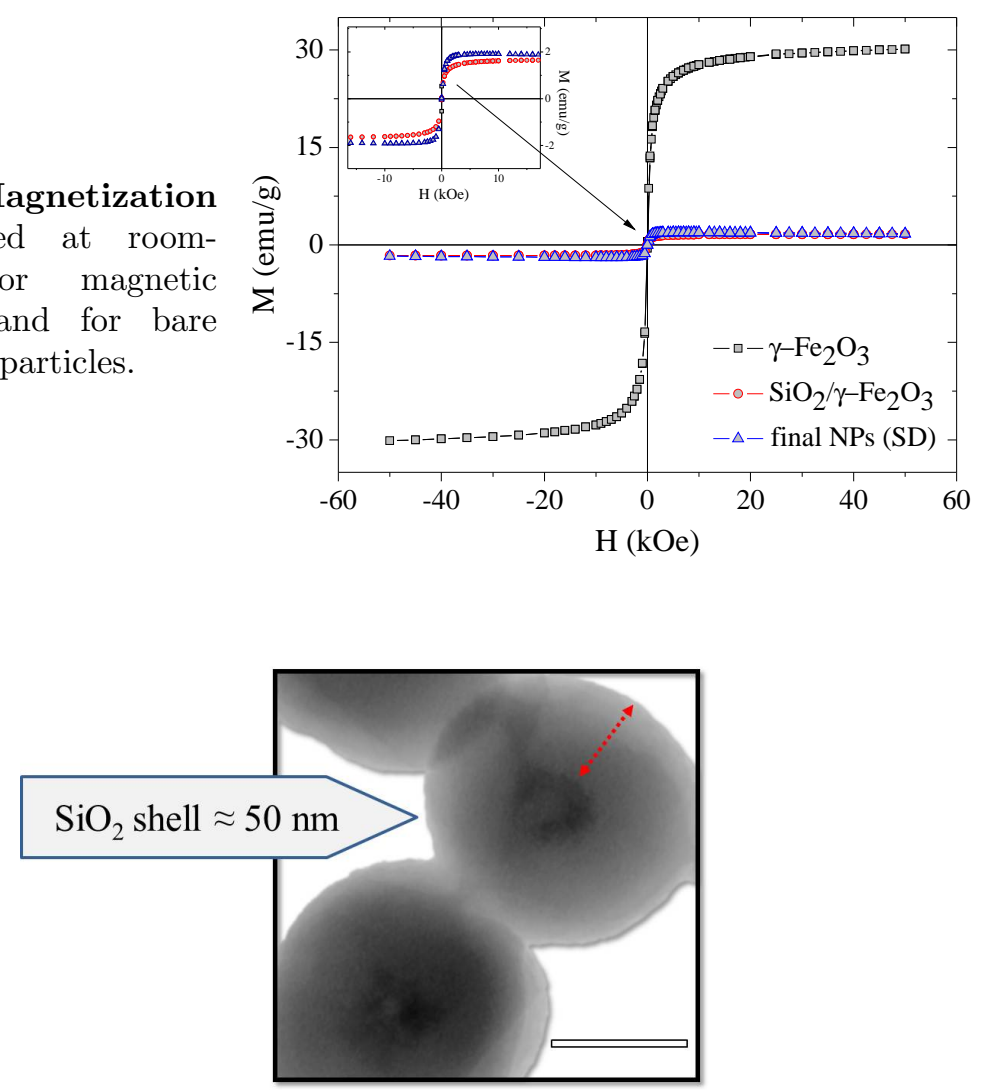

Figure 5.8: A TEM picture of magnetic silica spheres indicating the thickness of the silica shell onto maghemite nanoparticles, scale bar $-100 \mathrm{~nm}$.

is obviously more important for materials in the nm size range, where the surface/volume ratio is maximized. To this it must be added that the crystallinity could as well have an effect on magnetic characteristics (Feltin \& Pileni, 1997). If our particles contain even small amounts of non-magnetic impurities (hematite, for example), we can explain a significant reduction in the effective magnetic moment (Liao \& Chen, 2002). Needless to say, this explains the low saturation magnetization obtained for magnetic silica spheres at $50 \mathrm{kOe}(2.8 \mathrm{emu} / \mathrm{g})$, considering the thickness of the silica shell ( $\sim 50 \mathrm{~nm}$ Fig. 5.8), although the coating does not alter the superparamagnetic behaviour associated to the maghemite cores. 


\subsection{Magnetic Resonance Imaging}

MRI determinations were carried out in a $7 \mathrm{~T}$ Bruker Pharmascan (USA) thanks to the courtesy of professor S. Cerdán and doctor D. Calle, Universidad Autónoma de Madrid. The aim in these preliminary experiments was checking whether our nanocomposites could be useful as MRI contrast agents as well as drug delivery vehicles. The quantity characterizing this possibility is the relaxivity of the material: recall that this measures the relaxation times of the spins of the material tested in the MR spectrometer. At the concentrations normally employed in MR imaging, the impact of an MR contrast medium generally manifests on $T_{1}$ relaxation, leading to an increase of the initial relaxation time, which happens to be proportional to the concentration of the MR contrast medium. As a result, if $\mathrm{T}_{1} 0$ is the initial $T_{1}$ relaxation time and $R_{1}$ is the relaxivity, the $T_{1}$ relaxation time in the presence of the agent is given by:

$$
\frac{1}{T_{1}}=\frac{1}{T_{1} 0}+R_{1} C,
$$

where $C$ is the concentration of the MR contrast medium. Experiments were performed employing water and fetal calf serum (FCS) as dispersing phases. Prior to this, the amount of iron in the two samples tested (maghemite and maghemite/silica) was established by total X-ray reflection in a TXRF 8030C FEI spectrometer (Germany). Various suspensions of the original solution (Fe concentrations - $6575 \pm 14 \mathrm{mg} / \mathrm{mL}$ for bare maghemite solutions and $288.9 \pm 0.5 \mathrm{mg} / \mathrm{mL}$ in the case of silica coated maghemite nanoparticles) were placed in capillaries $1 \mathrm{~mm}$ in diameter in the isocenter of the magnetic field. Traditional weighting sequences were observed in $\mathrm{T}_{1}$ (longitudinal relaxation time, controlled by the spin environment), $\mathrm{T}_{2}$ (transversal or spin-relaxation time associated with the loss of phase between spins in the plane perpendicular to the orientation of the applied magnetic field), $\mathrm{T}_{2}^{*}$ (containing also field irregularities in the transversal relaxation).

The clinical goal of Magnetic Resonance Imaging is to modify the contrast and the acuteness/sharpness in detailed examination of body structures. This is connected with dissimilarities in relaxation times resulting from various tissues and, for specified magnetic field strength, imaging can be improved thanks to the generation of $\mathrm{T}_{2}$ and $\mathrm{T}_{2}^{*}$ contrast. The two quantities are therefore most helpful for applications of nanocomposites in MRI: due to the fact that $T_{2}$ time is longer when the water content is larger (tissues containing greater concentrations of water will become brighter in 


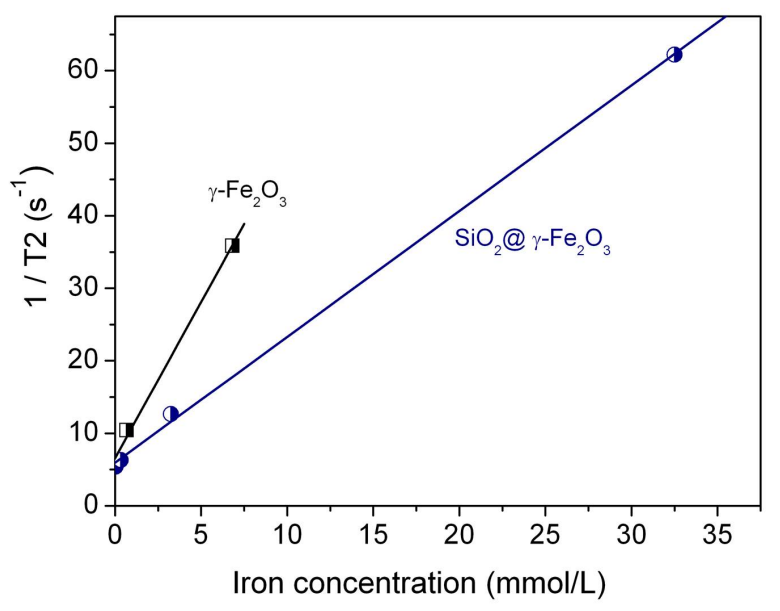

Figure 5.9: Spin-spin relaxation time for bare maghemite solution and its silica coating for distinct values of total Fe concentration in water.

case of $\mathrm{T}_{2}$ images) (Jezzard et al., 2002; Kuperman, 2000). More details can be found in (Browne \& Semelka, 1999; Prasad, 2006; Vlaardingerbroek $\&$ den Boer, 2003). In order to intensify contrast in this type of images gadolinium chelates exhibiting paramagnetic behaviour may be employed. They diminish $\mathrm{T}_{2}$ and $\mathrm{T}_{2}^{*}$ relaxation times, and consequently cause obscuring of the region where they are located. Still a better option is to use superparamagnetic nanoparticles, as those presented in this work.

Fig. 5.9 presents the decrease in $\mathrm{T}_{2}$ obtained with silica-coated maghemite and bare maghemite, and a comparable effect was determined on $\mathrm{T}_{1}$ and $\mathrm{T}_{2}^{*}$. It can be observed that there is a linear relationship between $1 / \mathrm{T}_{2}$ and the Fe concentration in the material and the slope of the linear trend is the relaxivity $\mathrm{R}$ in each case (See Fig. 5.9). Values of relaxivities obtained for $\mathrm{T}_{1}$ and $\mathrm{T}_{2}$ are presented in Tab. 5.1.

The relaxivity values are rather low when compared to the data published before concerning the relaxivity of $\mathrm{Fe}_{2} \mathrm{O}_{3}$ nanocompounds commercially available. This may be due to the fact that the majority of commercial products have magnetite as a basic nuclei. For instance, in a recent study reported $\mathrm{R}_{2}$ values were as large as $177.5 \mathrm{mM}^{-1} \mathrm{~s}^{-1}$ in case of maghemite nanoparticles with dextran coating, $358.9 \mathrm{mM}^{-1} \mathrm{~s}^{-1}$ for maghemite with carboxymethyl dextran coating composites and $124.5 \mathrm{mM}^{-1} \mathrm{~s}^{-1}$ for PEGphospholipid coated maghemite nanocomposites. All measurements were performed in the presence of fields on the order of $3 \mathrm{~T}$. These dissimilarities 


\begin{tabular}{cccc}
\hline & $\mathrm{R}_{1}$ & $\mathrm{R}_{2}$ & $\mathrm{R}_{2}^{*}$ \\
\hline Relaxivity & $\left(\mathrm{mM}^{-1} \mathrm{~s}^{-1}\right)$ & in Fetal & Calf Serum \\
\hline $\mathrm{Fe}_{2} \mathrm{O}_{3}$ & 0.0112 & 4.13 & 7.71 \\
$\mathrm{SiO}_{2} / \mathrm{Fe}_{2} \mathrm{O}_{3}$ & 0.0004 & 1.73 & 2.50 \\
\hline Relaxivity & $\left(\mathrm{mM}^{-1} \mathrm{~s}^{-1}\right)$ & in Water & \\
\hline $\mathrm{Fe}_{2} \mathrm{O}_{3}$ & 0.101 & 3.16 & 3.96 \\
$\mathrm{SiO}_{2} / \mathrm{Fe}_{2} \mathrm{O}_{3}$ & 0.0002 & 1.825 & 2.51 \\
\hline
\end{tabular}

Table 5.1: Values of $R_{1}, R_{2}$ and $R_{2}^{*}$ for bare maghemite and its silica-coating solutions measured in fetal calf serum and water.

may be explained taking into account that the particles in the above cited works were specifically developed as MRI contrast agents, while nanoparticles presented in this work are rather thought of as drug delivery vehicles, in which MR features would be just a supplementary or additional application. 



\section{Chapter 6}

\section{Capacity for antitumor drug vehiculization and tumor cell elimination}

In this chapter we describe the final application of the synthesized nanoparticles in the loading and delivery of antitumor drugs. As mentioned previously in this Thesis, the drug chosen for this proof-ofconcept experiments is doxorubicin. Here we show how do the particles adsorb this antitumor medicine and at what rate is the drug released. In addition, some examples are provided on the actual behavior of the drug-loaded particles in contact with liver and colon cancer cells. 

It is essential for antitumor drug action to access all drug-responsive cells with high enough concentration. In the case of anti-neoplasic drugs the goal is to ensure the maximum accumulation of these drugs within the target cells, as a way to obtain an increase of its therapeutic effectiveness. Moreover, new therapeutic strategies against cancer intend to reduce to a minimum the toxicity associated with anticancer therapy and avoid the development of cell resistance (Flórez, 2008). As previously mentioned, our work is based on the hypothesis that improvements in these fields are possible thanks to the use of magnetic nanovehicles for the drugs. Once the nanostructures have been described in previous Chapters it is essential to actually prove their usefulness as anticancer agents(Arias, 2008, 2011). Hence, our main purpose in this chapter is to employ our designed magnetic nanocompounds as antitumor drug systems. Particularly, we have investigated the vehiculization of doxorubicin (DOX). Among the large number of antineoplasic drugs, doxorubicin (Fig. 7.10) has demonstrated a notable limiting effect on the development of different tumors, as well as a substantial rise in viability of treated animals (Arcamone et al., 1997). Like other anthracyclines, DOX possesses a wide range of activity against human cancers (in fact, DOX belongs to the group of antitumor agents efficient in treatment of ten types of tumor with major occurrence: lung, stomach, breast, colon, cervix, head and neck, lymphoma, hepatobiliary system, esophageal tumor and prostate). Doxorubicin as a unique cytotoxic agent, has been shown to be very responsive in cases of advanced breast cancer, and it has demonstrated promising results in gastric carcinoma (a type of tumor on which only few cytotoxic agents are found to be active) (Kim \& Chu, 2000). DOX is also fundamental for the treatment of oesophageal carcinomas, solid tumors in children, osteosarcomas, Kaposi's sarcoma, Hodgkin and non-Hodgkin lymphomas and soft tissue sarcomas (Minotti et al., 2004). It is known that the very acidic pH in tumors may diminish the effectiveness of a number of anticancer therapies, as the activity of antitumor drugs which are weak bases like doxorubicin is restrained by low pH (Mahoney et al., 2003). Various processes like selection of apoptosisresisting phenotypes (Ohtsubo et al., 2001), straight effect of ion gradient on cytotoxic agent spreading and ion trapping (Raghunand \& Gillies, 2002) have been presumed to be the cause of this effect. The ion trapping mechanism states that cytotoxic agents being weak base will collect in more acidic parts of tumor. As a consequence, the low $\mathrm{pH}$ of tumors will effectually impede weakly basic agents from getting to their target within the cells, and 
as a consequence, it will decrease cytotoxicity.

Furthermore, the use of anthracyclines is limited by a chronic, cumulative, dose-related toxicity resulting in irreversible congestive heart failure. To prevent these secondary effects, the upper limit of proposed cumulative doses of anthracyclines were fixed at 500 or 450 to $600 \mathrm{mg} / \mathrm{m}^{2}$. The mechanisms through which anthracyclines provoke cardiotoxicity are believed to be different from those mediating their anticancer effectiveness. This idea is promising with regard to plan strategies for keeping the heart from being affected and at the same time not reducing tumor response (Minotti et al., 2004).

Polymer vehicles for doxorubicin may have the form of liposomes (Niu et al., 2010; Pakunlu et al., 2006; Rifkin et al., 2006) and polymeric micelles (Cuong et al., 2011; Gao et al., 2005; Kim et al., 2008; You et al., 2008). Nowadays there has been an enormous amount of research on the doxorubicin union with nanoparticulate carriers (Nawara et al., 2012; Du et al., 2011; Nowicka et al., 2009). This combination has resulted in controlled drug release over increased period of time, with the subsequent raise in efficiency and decrease in side effects (Jia et al., 2012; Prados et al., 2012). DOX has been found to be more efficient if attached to hydrophilic nanoparticles which are able to enter into the cell more profoundly than the cytotoxic agent alone, and therefore they can increase uptake of free anthracycline drug (Guo et al., 2008; Patil et al., 2008; Wang, 2007). Moreover, in order to overcome various problems concerning nonspecific DOX delivery, some targeted drug deliveries and combined therapies have been investigated (Brulé et al., 2011; Park et al., 2009). The significance of this drug can be measured by its widespread commercial use, involving different tradenames, both for the drug itself (for example, Adriamycin and Rubex are used for the hydrochloride salt, and the brand name of liposome- encapsulated doxorubicin is Doxil or Myocet).

In the present chapter we will carry out, in the first place, a spectrophotometic characterization for the determination of the capacity of the designed nanoparticulate system for the vehiculization of the antitumor drug. Moreover, we will determine the wavelength of the maximum absorbance and the molar absorbance coefficient for DOX. The reproducible methodology which allows to measure effectively the anti-neoplastic agent incorporation into nanoparticulate drug delivery systems is based on the Beer law. The study of surface drug adsorption by the two designed DOX delivery systems will be also evaluated qualitatively by means of electrophoretic mobility. Next, in vitro release analysis will be performed. Moreover, cell 
<smiles>COc1cccc2c1C(=O)c1c(O)c3c(c([OH2+])c1C2=O)C[C@@](O)(C(=O)CO)C[C@@H]3O[C@H]1C(N)=C(C)C(C)C1(N)O</smiles>

Figure 6.1: Chemical structure of cytotoxic agent, doxorubicin.

viability studies have been carried out for the second nanoparticulate drug delivery system. Finally, clear field microscopy has been employed in order to get a more illustrative information about cell viability.

\subsection{Stability of nanoparticulate drug delivery sys- tems}

It is important that the size is in the correct range, that is, the mentioned diameter is around that recommended for antitumor drug transport: particles with diameters below $500 \mathrm{~nm}$ can extravasate and accumulate in the tumor taking advantage of the enhanced permeation and retention effect (increased permeability in the parenchyma of the tumor vessels, and reduced clearance from the interstitial space in the tumor, see Refs. (Barreto et al., 2011; Decuzzi et al., 2010) for details. In addition, the synthesis of monodisperse nanoparticles is essential for achieving reliable and repeatable in vitro and cytotoxicity results (Mahmoudi et al., 2011b). Average hydrodynamic size values (estimated by DLS) for our nanocompounds were measured, and it was verified that the synthesized nanostructures do not undergo significant sedimentation or aggregation. Hydrodynamic particle diameters of bare maghemite and other nanostructures in water and culture medium are shown in Tab. 6.1. As anticipated, the small values of the standard deviation demonstrate that the nanoparticles are generally monodisperse.

The stability of the nanoparticulate solutions was directly examined 
112 Capacity for antitumor drug vehiculization and tumor cell elimination

\begin{tabular}{|c|c|c|}
\hline Nanoparticle type & \multicolumn{2}{|c|}{ Hydrodynamic size ( \pm S.D.)(nm) } \\
\hline & in water & in culture medium \\
\hline$\gamma-\mathrm{Fe}_{2} \mathrm{O}_{3}$ & $25 \pm 3$ & $42 \pm 8$ \\
\hline $\mathrm{SiO}_{2} / \gamma-\mathrm{Fe}_{2} \mathrm{O}_{3}$ & $202 \pm 14$ & $252 \pm 12$ \\
\hline $\operatorname{pol}(1) / \mathrm{SiO}_{2} / \gamma-\mathrm{Fe}_{2} \mathrm{O}_{3}$ & $260 \pm 30$ & $299 \pm 26$ \\
\hline $\mathrm{Au}_{S E M} / \operatorname{pol}(1) / \mathrm{SiO}_{2} / \gamma-\mathrm{Fe}_{2} \mathrm{O}_{3}$ & $265 \pm 19$ & $178 \pm 23$ \\
\hline $\mathrm{Au}_{L} / \operatorname{pol}(1) / \mathrm{SiO}_{2} / \gamma-\mathrm{Fe}_{2} \mathrm{O}_{3}$ & $202 \pm 16$ & $254 \pm 20$ \\
\hline $\operatorname{pol}(2) / \mathrm{SiO}_{2} / \gamma-\mathrm{Fe}_{2} \mathrm{O}_{3}$ & $222 \pm 20$ & $278 \pm 15$ \\
\hline $\mathrm{Au}_{S E M} / \operatorname{pol}(2) / \mathrm{SiO}_{2} / \gamma-\mathrm{Fe}_{2} \mathrm{O}_{3}$ & $259 \pm 12$ & $219 \pm 11$ \\
\hline $\mathrm{Au}_{L} / \operatorname{pol}(2) / \mathrm{SiO}_{2} / \gamma-\mathrm{Fe}_{2} \mathrm{O}_{3}$ & $269 \pm 15$ & $195 \pm 5$ \\
\hline
\end{tabular}

Table 6.1: Hydrodynamic diameter of the different nanoparticles from the first and the second type of drug carriers synthesized, when dispersed in water and in culture medium. Label - pol(1) refers to PDADMAC/ PSS/ PDADMAC polyelectrolytes combination, while pol(2) indicates APTMS.

by investigating the time evolution of their normalized optical absorbance (absorbance $\mathrm{cA}$ ) relative to its initial value $A_{0}$ ) at a wavelength of 489 $\mathrm{nm}$ for both types of DOX carriers, in water at physiological $\mathrm{pH}(7.4)$ and in culture medium. The results are included in Fig. 6.2. Both plots show that the ratio $A / A_{0}$ does not suffer important alterations after 166 minutes when nanostructures are dispersed in water. In the case of type I antitumor drug vehicles, stability in culture medium gradually and regularly decreases with time, whereas type II particles are observed to be much more stable, and their $A / A_{0}$ value does not diminish as much as in the previously discussed case. Note that there is a light rise in the absorbance value which may be due to the temporal agglomeration of the nanoparticles and this provokes that the absorbance value increases. No more than few percent decrease in absorbance is observed after the measurement time, being more unfavourable for the first DOX carriers design, indicating negligible particle aggregation and sedimentation. Hence, we can say that the systems presented may be both considered applicable for a sufficiently long time study, from the stability standpoint. 

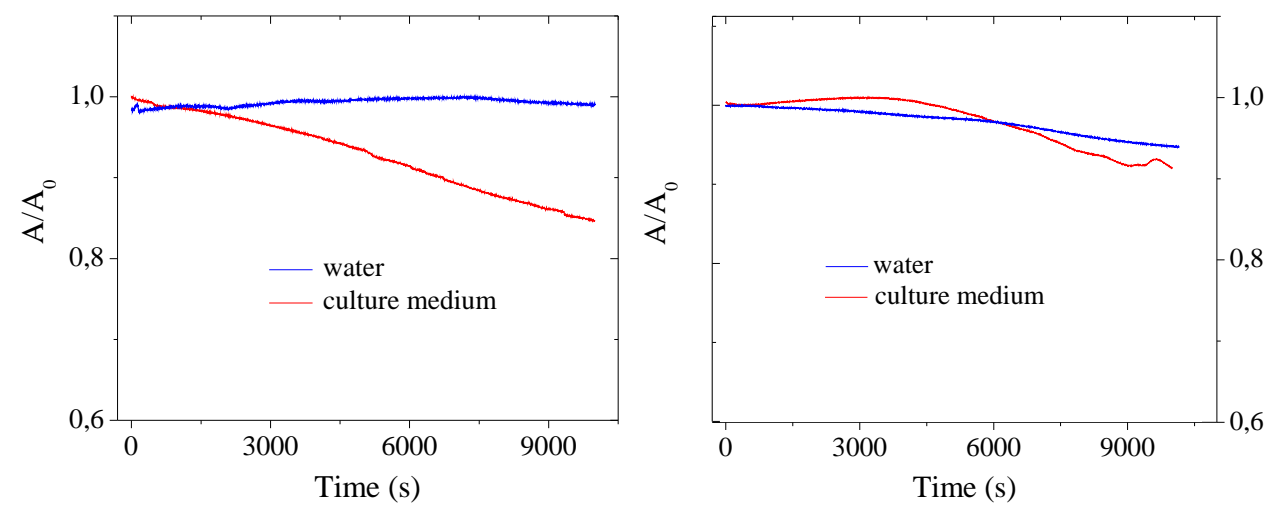

Figure 6.2: Optical absorbance $A$ expressed as a fraction of initial absorbance $A_{0}$ ) of final nanoparticulate suspensions as a function of time in water at pH 7.4 and in culture medium. Left: Final nanoparticulate system from the first design. Right: Final nanoparticulate system from the second design.

\subsection{UV-Vis spectrophotometry evaluation}

\subsubsection{Procedure for the experimental determination of the antitumor drug vehiculization}

Measurements of UV-Vis absorption spectra find an immense number of applications in the identification and quantitative determination of a great variety of organic and inorganic species. The spectroscopy of molecular absorption is based on the measurements of either transmittance $(T)$ or absorbance $(A)$ of solutions placed in a transparent cuvette with a pathlength of $l \mathrm{~cm}$. Usually, the concentration $c$ of the absorbing molecule has a linear relation with the optical absorbance of its solution, as shown below:

$$
A=-\log T=\log \frac{I_{0}}{I}=\varepsilon l c,
$$

where $\mathrm{I}_{0}$ and $I$ are the intensity of the incident and the transmitted light, respectively, and $\varepsilon$ is the molar absorption coefficient. This equation is known as Beer law (Skoog et al., 1998), and it has been demonstrated to be true for describing the absorption behaviour of a solution which contains small concentrations of absorbing species. In case of high concentrations, usually greater than $10^{-2} \mathrm{M}$, the average distance between molecules which are responsible for the absorption decreases to the point where ev- 
ery molecule alters the charge distribution of the neighbouring ones. This interaction may change the capacity of the molecules to absorb the radiation of a determined wavelength. Due to the fact that the magnitude of the interaction depends on the concentration, the manifestation of this phenomenon brings deviations from the linear relation between absorbance and concentration, in moderately concentrated solutions.

\subsubsection{Absorbance of anticancer drug solutions}

The UV-Vis 8500 spectrophotometer (DINKO Instruments, Spain) employed for our measurements possesses a deuterium lamp, which produces the continue spectrum useful for the region between 180 and $375 \mathrm{~nm}$, and a wolfram lamp, which is useful for the wavelengths region between 350 and $1100 \mathrm{~nm}$. In that way, we are able to obtain a spectrum from 180 and 1100 nm. A UV quartz cuvette with a pathlength of $1 \mathrm{~cm}$ was used for spectrophotometric evaluations. The first stages of spectrophotometric analysis pretend to establish work conditions and prepare the calibration curve which links absorbance with drug concentration. The absorbance measurements are normally performed at the wavelength of maximum absorbance, the most sensitive to concentration variations. Due to the light-sensitivity of the employed cytotoxic agent, an additional precaution is required in this case, which led us to keep the solutions away from ambient light, by wrapping all containers in aluminium foil. The natural $\mathrm{pH}$ of the solutions was always between 6 and 6.5 , and no effort was taken in changing it, until the in vitro release experiments, when the $\mathrm{pH}$ was 7.4 , provided by the buffer or the culture medium. All measurements for the calibration curve determination were carried out in duplicate, and DOX solutions in the concentration range $10^{-5} \mathrm{M}$ to $4 \times 10^{-4} \mathrm{M}$ were measured in the wavelength interval 200-700 $\mathrm{nm}$ at $1 \mathrm{~nm}$ steps.

Absorbance spectra are illustrated in Fig. 7.11, including only the wavelength range of interest in the spectra, namely, below $600 \mathrm{~nm}$. Two absorbance maxima are observed, although the one in the visible spectrum (489 $\mathrm{nm}$ ) changes in a more systematic way with concentration than does the UV maximum. Additionally, we note that for concentrations higher than $3 \times 10^{-4} \mathrm{M}$, the two peaks tend to merge, so the spectra are considerably deformed, and it will be clear that it is impossible to carry out concentration determinations in this range, as Beer's law is not fulfilled. Dilutions will be necessary in such conditions, as usual.

The results of the measurements are displayed in Tab. 6.2 as absorbance values of aqueous DOX solutions as a function of antitumor drug concentra- 


\begin{tabular}{c|c|c|c}
\hline Concentration $(\mathrm{M})$ & Absorbance & \pm S.D. & C.V. (\%) \\
\hline $10^{-5}$ & 0.1012 & 0.0015 & 1.5 \\
$2 \times 10^{-5}$ & 0.223 & 0.012 & 5.4 \\
$3 \times 10^{-5}$ & 0.342 & 0.008 & 2.5 \\
$4 \times 10^{-5}$ & 0.427 & 0.015 & 3.7 \\
$5 \times 10^{-5}$ & 0.550 & 0.014 & 2.6 \\
$6 \times 10^{-5}$ & 0.673 & 0.006 & 0.9 \\
$7 \times 10^{-5}$ & 0.762 & 0.009 & 1.2 \\
$8 \times 10^{-5}$ & 0.860 & 0.009 & 1.1 \\
$9 \times 10^{-5}$ & 0.970 & 0.005 & 0.5 \\
$10^{-4}$ & 1.067 & 0.005 & 0.44 \\
$1.1 \times 10^{-4}$ & 1.136 & 0.013 & 1.1 \\
$1.3 \times 10^{-4}$ & 1.309 & 0.024 & 1.9 \\
$1.6 \times 10^{-4}$ & 1.57 & 0.04 & 2.7 \\
$2 \times 10^{-4}$ & 1.87 & 0.05 & 2.7 \\
$3 \times 10^{-4}$ & 2.21 & 0.05 & 2.6 \\
$4 \times 10^{-4}$ & 2.32 & 0.023 & 1.8 \\
\hline
\end{tabular}

Table 6.2: Absorbance values (averages) for the aqueous solutions of doxorubicin with their corresponding standard deviations and coefficient of variation for every one of the concentrations indicated in the Table.

tion, for a wavelength of $489 \mathrm{~nm}$. The average absorbance value determined, with standard deviation (S.D.) values and the coefficient of variation (C.V.) are shown. The small C.V. values (in most cases $<5 \%$ ) indicates the adequate precision of the method. The linear fit of the absorbance $A$ vs. molar concentration $(C)[\mathrm{A}=(0.07 \pm 0.22)+(9400 \pm 220) \times \mathrm{C}]$ is statistically significant with a probability higher than $97 \%\left(\mathrm{R}^{2}=0.9702\right)$. From this, the molar absorption coefficient was estimated as $\varepsilon=9400 \pm 2200 \mathrm{~mol}^{-1} \mathrm{~cm}^{-1}$. Fig. 7.11 (bottom) displays the data and the calibration curve. It clearly demonstrates how absorbance values of different concentrations obtained at the wavelength of $489 \mathrm{~nm}$ are in accordance with the Beer lay.

\subsection{Antineoplastic agent incorporation}

Generally, there are two methods for vehiculization of an antitumor drug in the colloidal systems (Arias et al., 2010). The first one is its addition in the moment when the nanoparticles themselves are forming, so that the drug is 

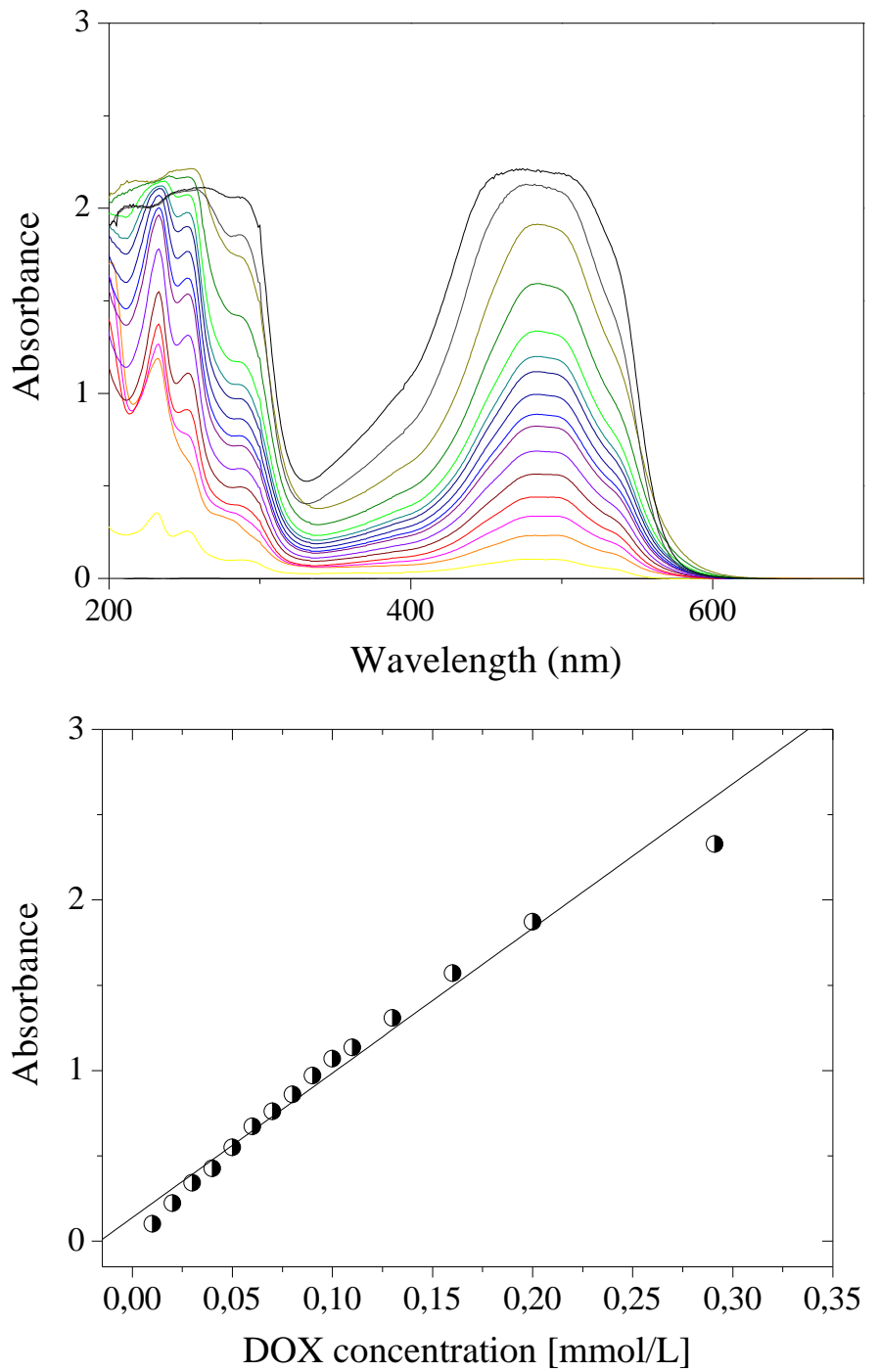

Figure 6.3: Top: UV-Vis absorbance spectrum of the aqueous DOX solutions. The molar concentrations of anti-neoplastic agent in an increasing order of absorbance are: $1 \mathrm{e}^{-5} \mathrm{M}, 2 \mathrm{e}^{-5} \mathrm{M}, 3 \mathrm{e}^{-5} \mathrm{M}, 4 \mathrm{e}^{-5} \mathrm{M}, 5 \mathrm{e}^{-5} \mathrm{M}, 6 \mathrm{e}^{-5} \mathrm{M}, 7 \mathrm{e}^{-5} \mathrm{M}$, $8 \mathrm{e}^{-5}, 9 \mathrm{e}^{-5} \mathrm{M}, 1 \mathrm{e}^{-4} \mathrm{M}, 1.1 \mathrm{e}^{-4} \mathrm{M}, 1.3 \mathrm{e}^{-4} \mathrm{M}, 1.6 \mathrm{e}^{-4} \mathrm{M}, 2 \mathrm{e}^{-4} \mathrm{M}, 3 \mathrm{e}^{-4} \mathrm{M}, 4 \mathrm{e}^{-4} \mathrm{M}$; Bottom: Absorbance as a function of doxorubicin concentration at the wavelength of $489 \mathrm{~nm}$. 
trapped inside the colloidal particle (absorption method). The second one is the surface adsorption from antitumor drug solution, after complete formation of the particles. This is the method used in this Thesis. The amount adsorbed will be controlled both qualitatively by means of electrophoretic evaluation of the surface charge evolution upon adsorption, and quantitatively, based on the spectrophotometric method above described.

\subsubsection{Spectrophotometric determination}

The quantitative follow-up of the surface adsorption of the antitumor agent onto the final nanocomposites was performed by first preparing $0.5 \mathrm{~mL}$ of doxorubicin solution containing $0.3 \mathrm{~mL}$ of final nanovehicles suspension and $0.2 \mathrm{~mL}$ of properly chosen DOX solutions so as to reach final molar concentrations of DOX, ranging from $1 \times 10^{-5}$ to $3 \times 10^{-4}$. The amount of nanoparticles in the obtained suspensions was $0.967 \mathrm{mg}$ in the case of first design and $0.625 \mathrm{mg}$ in the second one. The nanoparticles were left $24 \mathrm{~h}$ in contact with the antitumor agent in mild agitation at the temperature of $25 \pm 0.5^{\circ} \mathrm{C}$. After this time the nanoparticles were separated from the supernatant by centrifugation (14000 rpm, $20 \mathrm{~min}$ ), and next the absorbance of the supernatants was determined. The amount of antitumor drug incorporated (expressed in $\mu \mathrm{mol} / \mathrm{g}$ ) onto the surface of the final nanocomposites is determined in accordance with the equation presented below:

$$
\Gamma=\Delta C V_{t o t} 10^{6} / \mathrm{m},
$$

where $\Gamma$ represents the adsorption density, $\Delta C$ is the difference between the initial drug concentration and the equilibrium drug concentration, $\mathrm{V}_{\text {tot }}$ denotes the total volume of the solution and $m$ the mass of the particles. The obtained results are presented in Tab. 6.3.

The adsorption of DOX to both types of the final nanocomposites is shown by data in Fig. 7.12, where from the absorbance differences the drug loading efficiency for both kinds of nanovehicles can be obtained. Note that in the two designs the drug adsorption raises with the equilibrium concentration of antitumor drug in solution, until reaching saturation of the amount adsorbed. For the first DOX carriers (Fig. 7.12 top) the drug adsorption increases up to $10^{-4} \mathrm{M}$, where the saturation is almost attained at about $40 \mu \mathrm{mol} / \mathrm{g}$. As can be seen in Fig. 7.12 bottom, for the second DOX vehicles the adsorption also increases with equilibrium concentration of cytotoxic agent, reaching up to $80 \mu \mathrm{mol} / \mathrm{g}$ for the concentration range studied. 

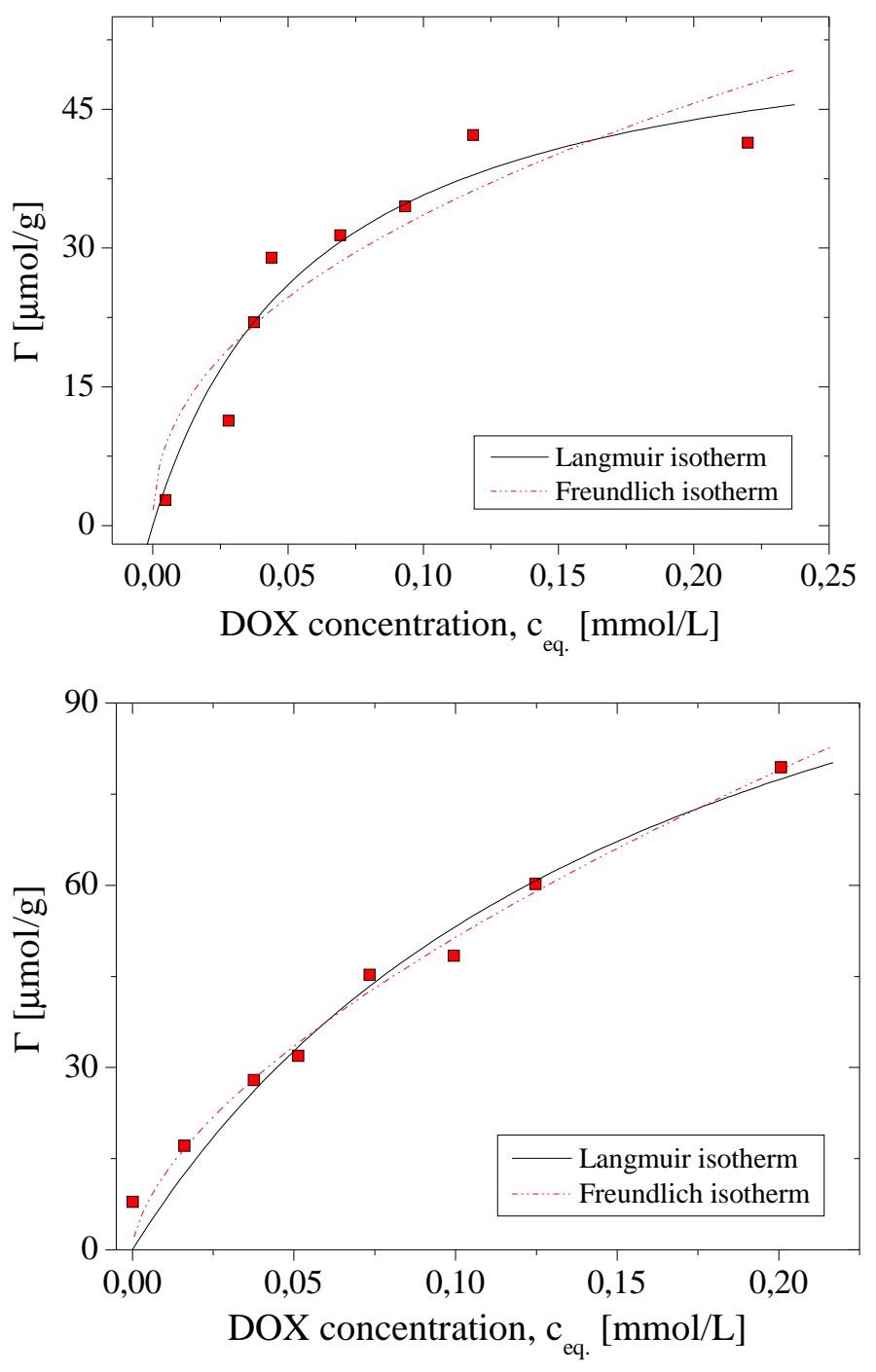

Figure 6.4: DOX adsorption on silica/gold-coated maghemite nanoparticles as a function of equilibrium concentration of DOX in solution. The solid lines are the best-fits to the Langmuir and Freundlich isotherms. Top: type I DOX vehicles; bottom: type II carriers. 


\begin{tabular}{ccccc}
\hline $\mathrm{A}_{489}$ & $\mathrm{C}_{\text {in }}[\mathrm{M}]$ & $\mathrm{C}_{\text {eq }}[\mathrm{M}]$ & $\Delta \mathrm{C}[\mathrm{M}]$ & $\Gamma[\mu \mathrm{mol} / \mathrm{g}]$ \\
\hline \hline First design & & & & \\
\hline 0.119 & $1 \times 10^{-5}$ & $4.73 \times 10^{-6}$ & $5.27 \times 10^{-6}$ & 2.75 \\
0.339 & $5 \times 10^{-5}$ & $2.81 \times 10^{-5}$ & $2.19 \times 10^{-5}$ & 11.33 \\
0.428 & $8 \times 10^{-5}$ & $3.75 \times 10^{-5}$ & $4.25 \times 10^{-5}$ & 21.96 \\
0.628 & $1 \times 10^{-4}$ & $4.41 \times 10^{-5}$ & $5.59 \times 10^{-5}$ & 28.94 \\
0.767 & $1.3 \times 10^{-4}$ & $6.93 \times 10^{-5}$ & $6.07 \times 10^{-5}$ & 31.38 \\
0.867 & $1.6 \times 10^{-4}$ & $9.33 \times 10^{-5}$ & $6.67 \times 10^{-5}$ & 34.50 \\
1.170 & $2 \times 10^{-4}$ & $1.18 \times 10^{-4}$ & $8.16 \times 10^{-5}$ & 42.20 \\
2.144 & $3 \times 10^{-4}$ & $2.20 \times 10^{-4}$ & $8.00 \times 10^{-5}$ & 41.38 \\
\hline Second design & & & & \\
\hline 0.076 & $1 \times 10^{-5}$ & $1.50 \times 10^{-7}$ & $9.85 \times 10^{-6}$ & 7.88 \\
0.227 & $5 \times 10^{-5}$ & $1.61 \times 10^{-5}$ & $3.39 \times 10^{-5}$ & 27.10 \\
0.429 & $8 \times 10^{-5}$ & $3.76 \times 10^{-5}$ & $4.24 \times 10^{-5}$ & 33.90 \\
0.558 & $1 \times 10^{-4}$ & $5.14 \times 10^{-5}$ & $4.86 \times 10^{-5}$ & 38.90 \\
0.766 & $1.3 \times 10^{-4}$ & $7.35 \times 10^{-5}$ & $5.65 \times 10^{-5}$ & 45.21 \\
1.010 & $1.6 \times 10^{-4}$ & $9.95 \times 10^{-5}$ & $6.05 \times 10^{-5}$ & 48.40 \\
1.250 & $2 \times 10^{-4}$ & $1.25 \times 10^{-4}$ & $7.52 \times 10^{-5}$ & 60.20 \\
1.960 & $3 \times 10^{-4}$ & $2.01 \times 10^{-4}$ & $9.92 \times 10^{-5}$ & 79.40 \\
\hline
\end{tabular}

Table 6.3: Values utilized in evaluation of the antitumor drug incorporation onto the surface of nanoparticles. 
The data profiles obtained from the study of antitumor adsorption at different concentrations were fitted to both Langmuir and Freundlich adsorption isotherms by using well-known adsorption isotherm equations. The Langmuir model (Langmuir, 1916, 1918) an empirical isotherm derived from a kinetic mechanism for the adsorption of gases on solids first proposed by this author. proposed kinetic mechanism. Using a notation suited to our purposes, the Langmuir isotherm model can be represented by the equation:

$$
\Gamma=\Gamma_{\max } \frac{k c}{1+k c},
$$

where $\Gamma$ denotes the adsorption density, $k$ is the equilibrium constant for the adsorption and crepresents the equilibrium concentration of doxorubicin. $\Gamma_{\max }$ represents the maximum adsorption capacity (monolayer coverage) which may reach the plateau in case of high equilibrium concentration of the adsorbate. In some cases, at lower concentrations, the Freundlich isotherm may better characterize the data (Freundlich, 1926). Boedeker was the first author who provided the empirical adsorption isotherm equation as presented below:

$$
\Gamma=k c^{n},
$$

and $k$ and $n$ are empirical (system specific) constants for each adsorbentadsorbate pair at a given temperature. This equation is known as the Freundlich adsorption isotherm because it was Herbert Freundlich who first employed this isotherm equation in his works, attributing a great meaning to it, and as a consequence, he popularized it. In particular, for the special case of heterogeneous surface energies, in which the energy term, $k$, varies as a function of surface coverage the use of the Freundlich model can be recommended (Zeldowitsch, 1934).

\subsubsection{Electrokinetic analysis}

It can be reasonably proposed that the principal mechanism of binding of cytotoxic agent to the final nanocomposites likely based on electrostatic attraction between the positive charge of dissolved doxorubicin (due to the protonation of the amino group of the DOX molecule), and the negative surface charge of gold nanoparticles forming the final layer of nanocompounds. For this reason, it can also be expected that the surface charge on the particles will be affected, a fact which can be confirmed indirectly but conclusively by means of electrokinetic techniques, electrophoresis in particular, considering the extreme sensibility of the electrophoresis technique 

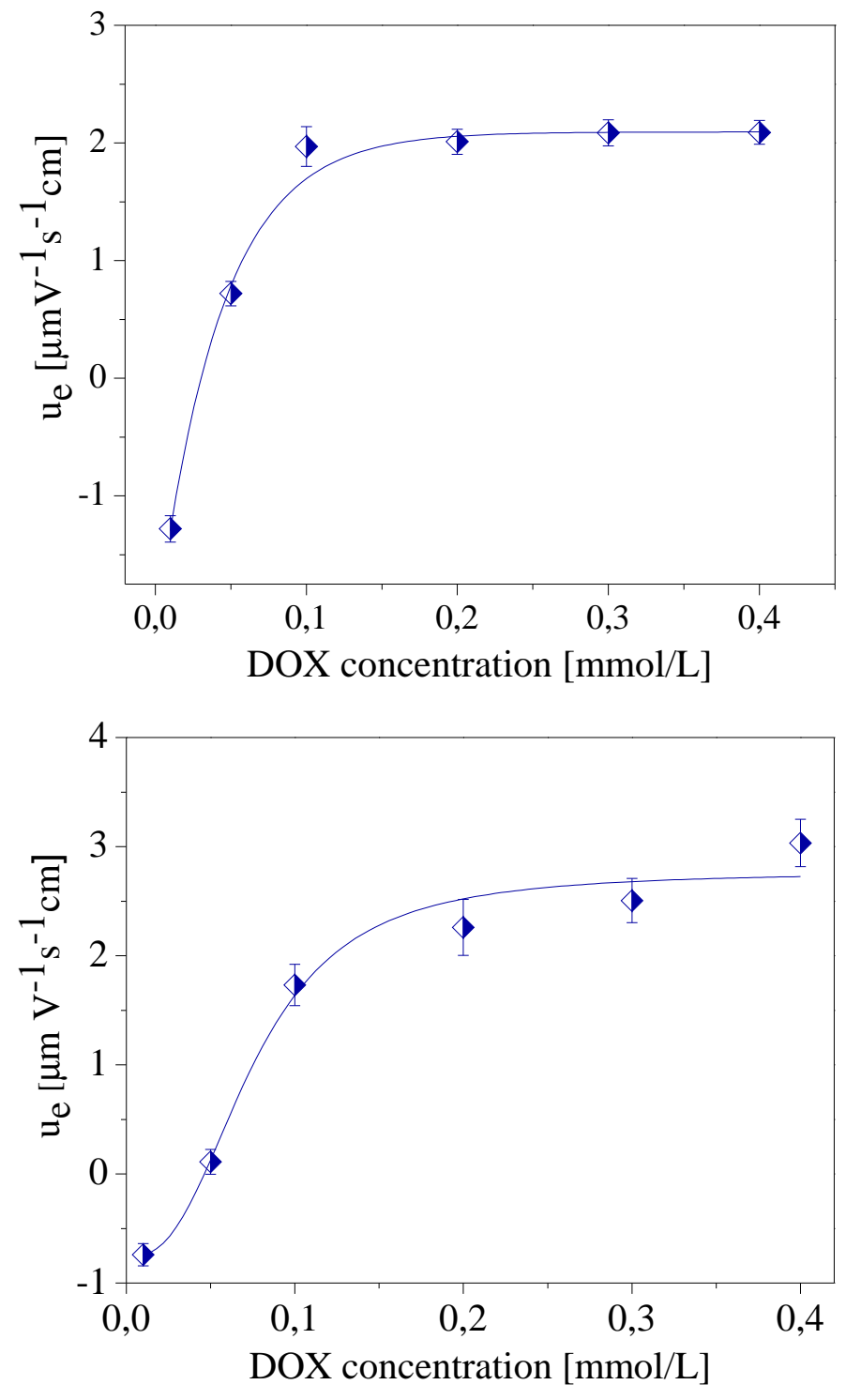

Figure 6.5: Electrophoretic mobility of maghemite/silica/gold nanostructures from the first design after $24 \mathrm{~h}$ contact with doxorubicin solutions of the concentrations indicated. Top: the first type of DOX vehicles; bottom: the second type of doxorubicin carriers. Both measurements were performed without fixing an ionic strength. 
122 Capacity for antitumor drug vehiculization and tumor cell elimination

\begin{tabular}{ccc}
\hline Parameters & First design & Second design \\
\hline Langmuir & & \\
\hline Maximum loading capacity $\Gamma_{\max }(\mu \mathrm{mol} / \mathrm{g})$ & $56.9 \pm 7.9$ & $141.7 \pm 24.6$ \\
Langmuir constant $(\mathrm{L} / \mathrm{mol})$ & $16.9 \pm 5.7$ & $6 \pm 2$ \\
Coefficient of determination $\left(\right.$ adj- $\left.\mathrm{R}^{2}\right)$ & 0.91 & 0.96 \\
\hline Freundlich & & \\
\hline Constant $(\mathrm{k})$ & $2 \pm 1.8$ & $0.44 \pm 0.1$ \\
Constant $(\mathrm{n})$ & $15 \pm 6.6$ & $0.62 \pm 0.04$ \\
Coefficient of determination $\left(\operatorname{adj}-\mathrm{R}^{2}\right)$ & 0.82 & 0.98 \\
\hline
\end{tabular}

Table 6.4: Calculated constants from Langmuir and Freundlich isotherms.

will allow to identify changes of the superficial electric properties of the final nanocompounds which will be the consequence of the antitumor drug adsorption.

The methodology used was the same as described in Chapter 3 for previous mobility measurements. Electrophoretic mobility measurements were carried out in a Zetasizer Nano-ZS (Malvern Instruments, U.K.). The suspensions with various DOX concentrations (from $1 \times 10^{-5}$ to $4 \times 10^{-4}$ $\mathrm{M})$ and without fixed ionic strength were prepared at their natural $\mathrm{pH}$. Specifically, $0.5 \mathrm{~mL}$ of the final nanocomposites mother suspensions was added to $1.5 \mathrm{~mL}$ of cytotoxic drug solution obtaining a $2 \mathrm{~mL}$ of slightly turbid solution suitable for this type of measurements. The solutions were left $24 \mathrm{~h}$ under mild agitation, and then the electrophoretic mobility was determined. For each suspension, 3 measuring runs were taken, with 3 cycles in each run. The temperature was $25.0 \pm 0.5^{\circ} \mathrm{C}$. The prepared suspensions were protected from the light with aluminium foil.

Fig. 7.13 shows the evolution of the electrophoretic mobility with antitumor drug concentration for the two types of DOX carriers. In both cases we can observe a general tendency of $u_{e}$ to increase (towards more positive values) as the antitumor drug concentration increases. This confirms the incorporation of the positively charged cytotoxic agent. As can be noticed, the mobility $u_{e}$ remains negative for both nanostructures only for solutions with low cytotoxic agent concentration. This is reasonable, since at such low DOX concentrations its adsorption is not enough to invert the sign of the electric charge of the final nanostructures, as the last layer prior to DOX adsorption is the gold layer quite negative at natural $\mathrm{pH}$. At higher concentrations charge inversion is observed, and, in fact, because of the pos- 
itive charge of DOX molecules in a wide $\mathrm{pH}$ range, their adsorption onto the negatively charged gold/maghemite nanostructures can be qualitatively followed. In the case of the first DOX vehicles, $u_{e}$ reaches a plateau starting at the DOX concentration of $0.2 \mathrm{mmol} / \mathrm{L}$, while for the second type of antitumor drug carriers we observe a slight but continuous rise of their $u_{e}$ value. Both results of adsorption density and electrophoretic characterization jointly considered confirm that the main mechanism of adsorption is electrostatic: the fact that the increase in concentration of DOX produces overcharging of the nanocomposites, the electrophoretic mobility becoming increasingly positive as more DOX is in solution, is an indication of strong interaction. Similar conclusions were reached recently by (Tapeinos et al., 2013), who described the adsorption of this drug as a test of poly(glycidyl methacrylate) microspheres as drug vehicles.

\subsection{In vitro doxorubicin drug release}

\subsubsection{Methodology}

Drug release. $0.6 \mathrm{~mL}$ of solution of the final nanocomposites (containing, $\approx 0.1 \%$ wt and $0.065 \%$ wt solid content for the first and the second DOX vehicle design, respectively) was added to $0.4 \mathrm{~mL}$ of $0.75 \mathrm{mM}$ DOX solution to a final drug concentration of $0.3 \mathrm{mM}$, and left to stay during $24 \mathrm{~h}$ under agitation. Initially, the suspension was centrifuged twice (20 min. at 14000 $\mathrm{rpm}$ ) in order to get rid of the supernatant including the non-adsorbed antitumor drug. Later, the nanoparticles were re-dispersed in $1 \mathrm{~mL}$ of PBS $(0.15 \mathrm{M})$ and kept at $37{ }^{\circ} \mathrm{C}$ while the release was taking place. At the indicated time intervals, the sample was centrifuged during 2 min at 14000 $\mathrm{rpm}, 150 \mu \mathrm{L}$ of the supernatant was extracted and the absorbance of this taken sample was measured in the Dinko 8500 spectrophotometer set at the wavelength of $489 \mathrm{~nm}$. The same volume was then given back to the releasing suspension.

Cell culture experiments. Two well-characterized human cancer cell lines, namely, PLC-PRF-5 (human liver cancer cell line which synthesizes hepatitis B virus surface antigen (HgsAB)), and DLD-1 (colorectal adenocarcinoma cell line), were maintained as an adherent monolayer in the culture media RPMI-1640 and DMEM (Invitrogen, USA), respectively, supplemented with $10 \%$ fetal bovine serum (FBS, JRH Biosciences, USA), $100 \mathrm{U} / \mathrm{mL}$ penicillin and $100 \mu \mathrm{g} / \mathrm{mL}$ streptomycin (Sigma Aldrich) and incubated at $37^{\circ} \mathrm{C}$ in $5 \% \mathrm{CO}_{2}$ atmosphere. For the cell culture experiments, cells were grown in chamber slides ( 8 wells Chamber Slide, Sigma-Aldrich) 

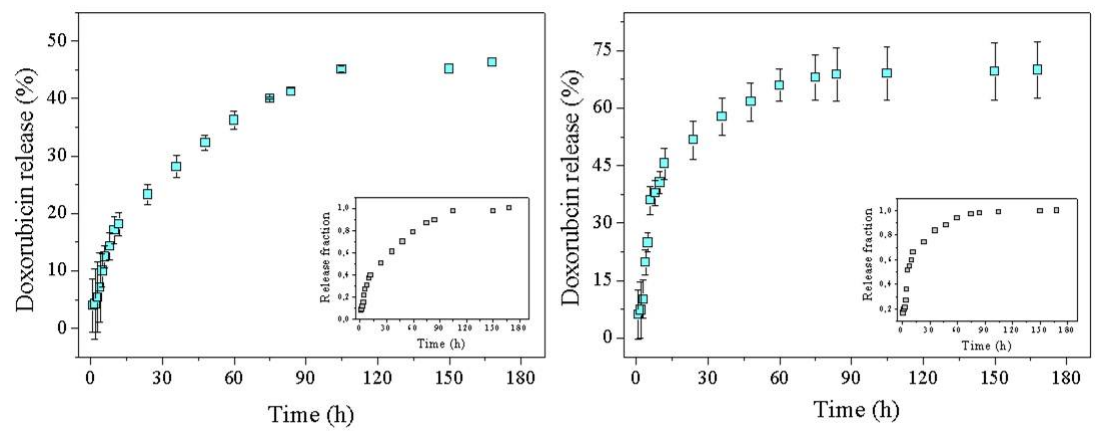

Figure 6.6: Doxorubicin release profile from magnetic nanocomposites Left:the first type of DOX nanoparticulate carriers; Right: the second type of DOX vehicles. Both graphs contain insets with the corresponding released fraction of the cytotoxic agent.

and treated with different dilutions of, respectively, doxorubicin, nanocomposites, and doxorubicin adsorbed onto nanocomposites for 2 hours in the corresponding culture medium supplemented with $10 \%$ FBS at $37{ }^{\circ} \mathrm{C}$. After treatment, the cells were fixed in ice-cold paraformaldehyde (4\%) for 10 minutes and nuclear counterstained with DAPI (4'-6-Diamidino-2phenylindole, Sigma Aldrich). Nanoparticle autofluorescence was visualized with a Leica Spectral confocal laser microscope, and DAPI-stained samples staining were observed with a Leica Fluorescence Microscope (Bensheim, Germany) using a $63 \mathrm{X}$ oil-immersion objective.

\subsubsection{Results and discussion}

Fig. 7.14 shows the antitumor drug release (\% of total drug on the particles) as a function of time for two types of DOX vehicles. The in-vitro release was performed for a drug concentration of $2 \times 10^{-4} \mathrm{M}$.

There are many kinetic models that characterize the complete release of the cytotoxic agent from the dosage forms. The utilization of in vitro drug dissolution results in order to predict in vivo bio-distribution may be thought to be the reasonable progression of controlled release drug delivery systems (Dash et al., 2010). Drug delivery systems will deliver the cytotoxic agent at a rate determined by the needs of the body area over a specified period of time. Control in release is useful for maintaining constant levels of antitumor drug in the target tissues or cells. The techniques of approximation to study the kinetics of cytotoxic agent release may be categorized as follows: 
- Statistical methods, such as exploratory data analysis techniques, repeated measures design, multivariate approach - MANOVA (multivariate analysis of variance) (Mauger et al., 1986; Polli et al., 1997).

- Model dependent methods, like zero order, first order, Higuchi approach, Korsmeyer-Peppas model, Hixson Crowell model, Baker-Lonsdale model, Weibull model, etc. (Costa \& Lobo, 2001; Shah et al., 1997).

- Model independent methods, for example difference factors $\left(\mathrm{f}_{1}\right)$, similarity factor $\left(\mathrm{f}_{2}\right)$ (Costa, 2001; Moore \& Flanner, 1996).

Model dependent methods are characterized by various mathematical functions, which depict the dissolution profile. Among the models presented above the most interesting from our point of view are:

Zero-order model describes the situation where the cytotoxic agent dissolution from dosage forms does not provoke separation into different/unequal parts and the drug release occurs at a low rate, according to the equation:

$$
Q_{0}-Q_{t}=K_{0} t
$$

where $Q_{t}$ represents the amount of cytotoxic agent dissolved in time $t, Q_{0}$ is the initial content of antitumor drug present in the solution (frequently $Q_{0}=0$ ), and $K_{0}$ is the zero order release constant represented in units of concentration/time. The results obtained must be represented as cumulative amount of agent released as a function of time in order to observe the release kinetics. This kind of relation may be employed to characterize the cytotoxic agent dissolution of various kinds of changed release pharmaceutical dosage forms. For example, it may be applied for transdermal drug delivery systems, like matrix tablets with almost insoluble agents in coated forms (Freitas \& Marchetti, 2005).

First order model has been also employed to characterize absorption and/or elimination of some agents, although it is not easy to explain its observations on theoretical grounds. The release of the cytotoxic agent in this case may be represented as:

$$
\frac{C}{t}=-K C
$$

where $\mathrm{K}$ represents first order rate constant (in units of time ${ }^{-1}$ ). Eq. 7.1 may be also represented as:

$$
\log C=\log C_{0}-\frac{K t}{2.303},
$$


and $C_{0}$ is the initial concentration of cytotoxic agent, and $k$ represents first order rate constant. In order to study the drug release kinetics, the results are plotted as log (cumulative percentage of agent which is left) versus time. One can expect a straight line having a slope of $-K / 2.303$. The above relation may be also expressed as:

$$
\log Q_{t}=\log Q_{0}+\frac{K_{t} t}{2.303}
$$

Then a graph of the decimal logarithm of the dissolved amount of DOX vs time will be linear. This equation may be employed for characterizing antitumor drug dissolution in pharmaceutical dosage forms like those with water-soluble agents in porous matrices.

The Higuchi model was the first mathematical model for characterizing drug release from a matrix system published by prof. Takeru Higuchi in 1961 (Higuchi, 1961). At the beginning it was thought to be applicable to planar systems, and then it was extended to include various geometries and porous matrices (Desai et al., 1965; Higuchi, 1963; Lapidus \& Lordi, 1966). In his first contribution Higuchi imagined the drug release as being modelled by the release of a cytotoxic agent from a thin ointment base, according to the following conditions:

a) The cytotoxic agent transport via the ointment film is restricted by rate, while cytotoxic drug transport inside the skin is quick;

b) The skin plays a role of a perfect sink: the concentration of the antitumor drug here is insignificant;

c) The initial concentration of cytotoxic agent is much higher than its solubility;

d) The antitumor agent is homogeneously distributed in every point of the ointment base at the beginning of the release process;

e) Cytotoxic agent diffusion occurs only in one dimension - the surface of the ointment base exposed to the film is extensive when compared to its thickness (edge effects must be insignificant);

f) Agent molecules are considerably smaller than drug delivery system thickness (the agent is excellently dispersed in the ointment base);

g) Swelling or dissolution of matrix (ointment film) are considered insignificant during drug release;

h) The diffusivity of the antitumor agent is constant (it does not depend on eiher time or the location in the film);

i) Perfect sink conditions must be constantly fulfilled in the release surroundings. 
In accordance with the model, Higuchi equation is represented as:

$$
\frac{M_{t}}{A}=\sqrt{D\left(2 C-C_{s}\right) C_{s} t},
$$

where $M_{t}$ is the amount of cytotoxic agent released in time $t$ per unit area $A, C$ represents the initial concentration of antitumor agent and $C_{s}$ is the agent solubility in the matrix medium. $D$ represents diffusivity of the agent molecules (diffusion coefficient) in the matrix.

In case of an important initial excess of the cytotoxic agent $\left(C \gg C_{s}\right)$ the above equation may be expressed as:

$$
M_{t}=A \sqrt{2 C D C_{s} t}
$$

This equation represents the classical Higuchi model. Certainly, one cannot infringe the rules on which origin of this well-known model is based. Especially, the pseudo-steady-state approximation must be valid, demanding a high initial excess of cytotoxic agent and also an unchanging "ointmentskin"(no swelling and no dissolution of the matrix). Clearly, the Higuchi equation may be also employed to characterize antitumor drug release from different drug delivery systems not only ointment bases, for example thin patches or thin films. In addition, it has been further extended to different geometries (Higuchi, 1963; Lee, 2011; Roseman \& Higuchi, 1970). We note that Eq. 6.10 may be also expressed as follows (in more simplified form):

$$
\frac{M_{t}}{M_{\infty}}=k \sqrt{t}
$$

where $M_{\infty}$ is the absolute cumulative amount of antitumor agent released at infinite time (that should be the same as the absolute amount of antitumor drug attached to the system at time $t=0$ ), and $k$ is a constant including the design variables of the drug delivery system.

$$
k=A \sqrt{2 C D C_{s}}
$$

Therefore, as can be observed, the classical Higuchi model characterizes a "square root of time" kinetics of the release process. Nevertheless, it must be indicated that the constant $k$ possesses a very particular and physically practical meaning for the Higuchi model. Regrettably, this constant is not always considered and in some cases the classical Higuchi model is confused with different kinds of release kinetics of square root of time. It must be emphasized that different types of drug delivery systems, that are controlled by release mechanisms distinct from that of Higuchi model, may be as 
well described by a proportionality between the cumulative quantity of cytotoxic agent and the square root of time. As an example, we can present a proportional relation between the fractional quantity of released cytotoxic agent and the square root of time derived from an exact solution of Fick's second law of diffusion for thin films with thickness $(\delta)$ under perfect sink conditions. In this case, considering $C<C_{s}$ and taking into consideration constant diffusivities (Baker \& Longsdale, 1974; Crank, 1975):

$$
\frac{M_{t}}{M_{\infty}}=4\left(\frac{M_{t}}{M_{\infty}}\right)^{1 / 2}\left\{\pi^{-1 / 2}+2 \sum_{n=1}^{\infty}(-1)^{n} \operatorname{ierfc} \frac{n \delta}{2 \sqrt{D t}}\right\}
$$

In this equation, $M_{t}$ and $M_{\infty}$ denote the absolute cumulative amount of cytotoxic agent released at time $t$ and infinite time, respectively, and $D$ is the diffusivity of the cytotoxic agent inside the polymeric system. Due to the fact that the second term in the second brackets disappears at short times, a precise enough estimation of Eq. 6.13 when $M_{t} / M_{\infty}<0.6$ may be presented as:

$$
\frac{M_{t}}{M_{\infty}}=4\left(\frac{D t}{\pi \delta^{2}}\right)^{1 / 2}=k^{\prime} \sqrt{t},
$$

and $\mathrm{k}^{\prime}$ is a constant. Therefore, a proportional relation between the fraction of anticancer drug released and the square root of time may be as well supported on these physical conditions that are considerably distinct from those investigated by Higuchi. Nevertheless, diffusion is the governing mechanism for both examples and therefore a $\sqrt{t}$-dependence is usually considered as an indication of diffusion-controlled drug release processes.

The Hixson-Crowell model was elaborated in 1931. It is based on the existence of a proportionality relationship between the regular area of the particles and the cube root of its volume (Hixson \& Crowell, 1931), that is::

$$
W_{0}^{1 / 3}-W_{t}^{1 / 3}=\alpha t,
$$

where $W_{0}$ represents the initial amount of cytotoxic agent in the pharmaceutical dosage form, $W_{t}$ is the quantity of drug left in the pharmaceutical dosage form at time $t$ and $\alpha$ represents a constant enclosing the surface-volume relation. This model characterizes the release process from drug delivery systems where exists some alteration in particle (or tablet) size and, as a consequence, on its surface area. In order to analyse the 
results, data obtained must be represented as the cubic root of antitumor agent percentage left in the matrix as a function of time (Chen et al., 2007). Such a plot will be linear, as long as the system has not come to the equilibrium conditions, and the shape of the pharmaceutical dosage remains the same, while it becomes proportionally smaller over time. Use of this model is based on the hypothesis that the release speed is restricted by the antitumor agent particles dissolution rate and not by the diffusion which may take place through the polymeric matrix. The Hixson-Crowell model has been employed to characterize the release profile keeping in mind the decreasing surface of the particles during the dissolution (Prista et al., 1995).

The Korsmeyer-Peppas model has its origins in an equation obtained by Korsmeyer in 1983 which characterizes a drug release process (Korsmeyer et al., 1983). In order to determine the mechanism of antitumor agent release process, during the initial $60 \%$ agent release, results are fitted in Korsmeyer-Peppas equation (Ritger \& Peppas, 1987a,b), which is expressed as:

$$
M_{t} / M_{\infty}=k t^{n},
$$

and $M_{t} / M_{\infty}$ represents the fraction of antitumor agent released at time $t, k$ is the release rate constant and $n$ represents the release exponent, suggesting the mechanism of drug release. This equation is also known as the power law. As can be noticed, the classical Higuchi model (Eq. 6.10) is similar to the above presented short time estimation of the exact solution of Fick's second law for thin layers: Eq. 6.14 symbolizes the special example of the so-called power law where $n=0.5$. Peppas et al. were the first who proposed an introductory explanation for the use and the restrictions of this equation (Peppas, 1985). Therefore, Eq. 7.2 possesses two different physical practical meanings, first when $n=0.5$ pointing out diffusion-controlled drug release, and when $n$ is equal to 1 signalling swelling-controlled drug release. In case when $n$ exponent has a value between 0.5 and 1 , is may be considered as an indication of the superposition of both drug release mechanisms (anomalous transport). One must remember that the two $n$ values mentioned above $(n=0.5$ and $n=1)$ are only acceptable for thick flat objects. In case of spheres and other geometries distinct values have been obtained (Siepmann \& Peppas, 2001; Ritger \& Peppas, 1987a), as presented in Tab. 6.5.

In order to determine the $n$ exponent, only the part of the release graph where $M_{t} / M_{\infty}<0.6$ should be employed. To investigate the kinetics of the release process, the results achieved should be represented as log cumulative 
130 Capacity for antitumor drug vehiculization and tumor cell elimination

\begin{tabular}{ccc}
\hline Release exponent $(\mathrm{n})$ & Drug transport mechanism & Rate as $y=f(t)$ \\
\hline Thin Film & & \\
\hline 0.5 & Fickian diffusion & $t^{-0.5}$ \\
$0.5<\mathrm{n}=1.0$ & $\begin{array}{c}\text { Non-Fickian (Anomalous) transport } \\
\text { Case II (relaxational) transport }\end{array}$ & Zero order release \\
1.0 & & \\
\hline Sphere & Fickian diffusion & $t^{-0.5}$ \\
\hline 0.43 & Non-Fickian transport & $t^{n-1}$ \\
$0.43<\mathrm{n}=0.85$ & Case II (relaxational) transport & Zero order release \\
\hline 0.89 & Fickian diffusion & $t^{-0.5}$ \\
\hline Cylinder & Non-Fickian transport & $t^{n-1}$ \\
0.5 & Case II (relaxational) transport & Zero order release \\
$0.45<\mathrm{n}=0.89$ & Super case II transport & $t^{n-1}$ \\
Higher than 0.89 & . &
\end{tabular}

Table 6.5: Explanation of diffusional release profile from polymers (Siepmann \& Peppas, 2001).

percentage of antitumor agent released as a function of $\log t$.

A general empirical equation derived by Weibull in 1951 was changed into the dissolution/release mechanism equation (Lagenbucher, 1972). After that, it can be applied in a successful manner to nearly every release curve (Goldsmith et al., 1978; Vudathala \& Rogers, 1992). The Weibull model is expressed by the equation:

$$
M=\left[1-e^{\frac{(t-T)^{b}}{a}}\right]
$$

$\mathrm{M}$ in this model represents the accumulated fraction of cytotoxic agent in the solution as a function of time $t . T$ represents the lag time, required for the beginning of the release process and in almost all cases is zero. The parameter $a$ symbolizes a scale variable which characterizes the time dependence, and $b$ denotes the shape parameter of the dissolution curve progress. The Weibull equation is more practical in case of comparisons of release profiles of matrix drug delivery systems (Lagenbucher, 1972).

The kinetics of antitumor drug release for the first and the second type of DOX carriers is presented in Fig. 7.14. Note that up to $46 \%$ and $70 \%$ of the antitumor drug for the first and the second DOX vehicles, respectively, can be released after $168 \mathrm{~h}$. The kinetics profile achieved suggests 

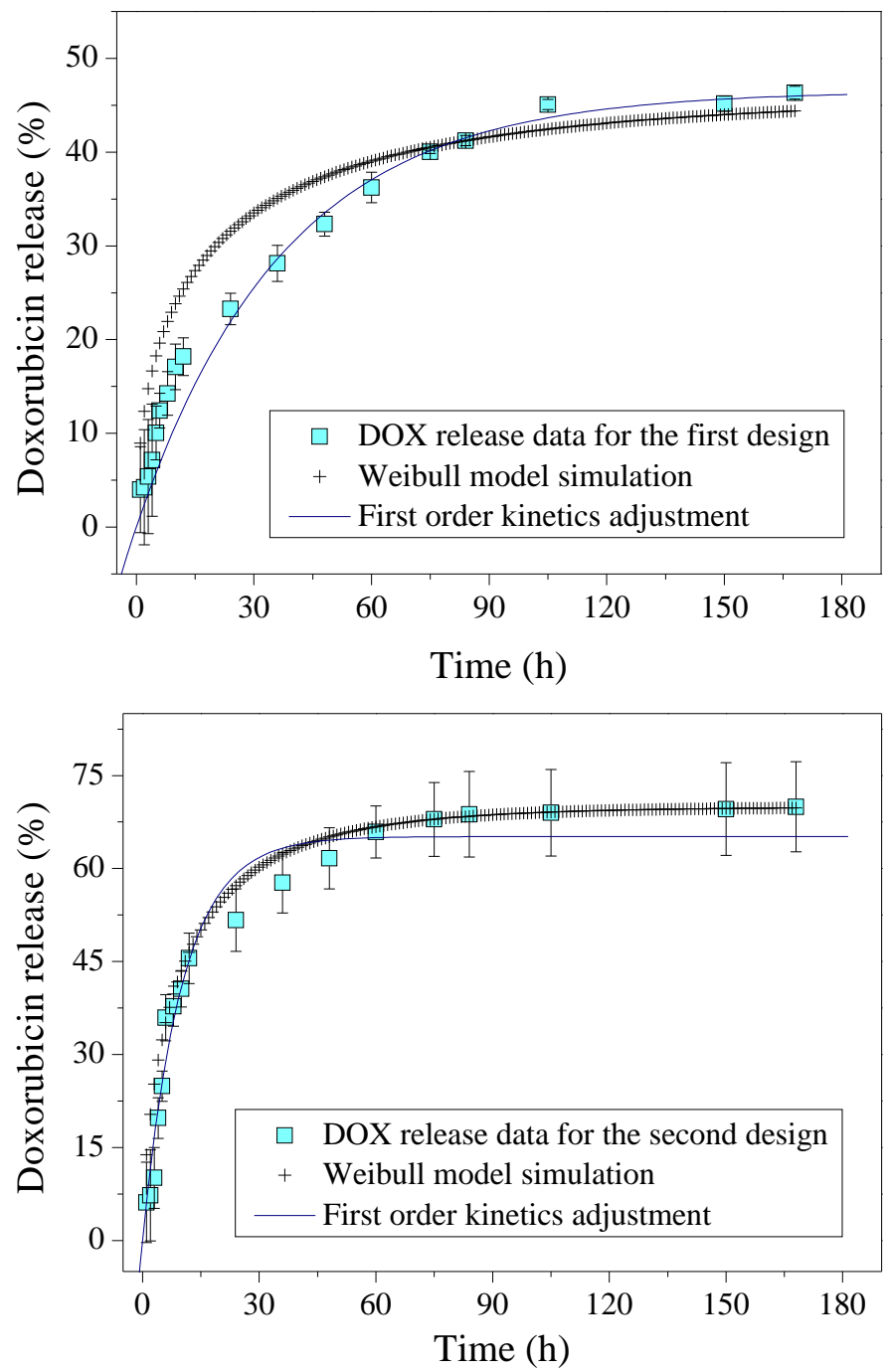

Figure 6.7: Doxorubicin release profile from magnetic nanocomposites Top:the first type of DOX nanoparticulate carriers. bottom: the second type of DOX vehicles. The solid lines are the best fits of the release data to a first order kinetics. For the two types of DOX vehicles, both graphics present Weibull model simulation with the parameters obtained from the fit of the released data to a Weibull model. 
the mechanisms of drug release from the nanocarriers. The mathematical models are used to evaluate the kinetics and mechanism of drug release from the drug delivery systems. The model that best fits the release data is selected based on the adjusted coefficient of determination $\left(\operatorname{adj}-\mathrm{R}^{2}\right), \mathbf{F}$ statistics value and Akaike Information Criterion (AIC) obtained from the release data fittings to the various kinetic models. Note that the coefficient of determination $\left(\mathrm{R}^{2}\right)$ is usually employed in order to estimate the best model of those applicable to "fit" a set of data. Nevertheless, $\mathrm{R}^{2}$ is predisposed to increase when more parameters are added, without regard to the importance of the variable added to the model; for this reason, it is suitable for comparing fittings from models with identical number of adjustable parameters. When contrasting models with distinct numbers of parameters, an adjusted determination coefficient is more significant and adequate. This modified parameter, adj- $\mathrm{R}^{2}$ is expressed by:

$$
\operatorname{adj}-\mathrm{R}^{2}=1-\frac{(n-1)}{(n-p)}\left(1-R^{2}\right),
$$

where $n$ is the number of the release data points and $p$ is the number of parameters in the model. Note that adj- $\mathrm{R}^{2}$ in fact may become smaller when adding new fitting parameter, in that way indicating if the addition of the new parameter increases the quality of the model or may result in overfitting. The model which gives higher adj- $\mathrm{R}^{2}$ and $\mathbf{F}$ values is considered as the best fit of the release data. The contrary situation occurs for AIC, for which the best fit corresponds to the AIC value with lowest absolute value. This has become one of the most widely used methods. The criterion AIC is calculated as follows:

$$
\mathrm{AIC}=n \times \ln (\mathrm{WSSR})+2 \times p
$$

where $n$ is the number of dissolution data points $(\mathrm{M} / \mathrm{t}), p$ is the number of the parameters of the model, WSSR represents the weighed sum of square of residues.

Tab. 6.6 displays the values of the release data fitting to the described models. Considering the adjusted determination coefficient $\left(\operatorname{adj}-\mathrm{R}^{2}\right)$, the $A I C$ and $F$ values obtained from fitting of the kinetic models to the release data (See Tab. 6.6), it can be clearly stated that the best fit for the release data in case of both types of drug carriers is first order kinetics (See Fig. 7.15). This good fit suggests that these nanosystems release the antitumor agent at a rate proportional to the amount of drug remaining in their internal part (e.g., like water-soluble drugs in porous matrices) or 


\begin{tabular}{|c|c|c|}
\hline Parameters & First design & Second design \\
\hline \multicolumn{3}{|c|}{ Zero-order model } \\
\hline $\mathrm{k}$ & 0.454 & 0.776 \\
\hline $\operatorname{adj}-R^{2}$ & 0.94 & 0.59 \\
\hline $\mathrm{F}$ & 61 & 30 \\
\hline $\mathrm{AIC}$ & 103 & 71 \\
\hline \multicolumn{3}{|c|}{ First order model } \\
\hline $\mathrm{k}$ & $0.02 \phi$ & 0.099 \\
\hline $\operatorname{adj}-R^{2}$ & $0.96 \$$ & 0.962 \\
\hline $\mathrm{F}$ & 2735 & 31196 \\
\hline $\mathrm{AIC}$ & 23 & -0.05 \\
\hline \multicolumn{3}{|c|}{ Higuchi model } \\
\hline $\mathrm{k}$ & 4.44 & 8.34 \\
\hline $\operatorname{adj}-R^{2}$ & 0.591 & 0.553 \\
\hline $\mathrm{F}$ & 747 & 5270 \\
\hline $\mathrm{AIC}$ & 64 & 42 \\
\hline \multicolumn{3}{|c|}{ Hixson-Crowell model } \\
\hline $\operatorname{adj}-R^{2}$ & 0.672 & 0.447 \\
\hline $\mathrm{F}$ & 36 & 15 \\
\hline $\mathrm{AIC}$ & -189 & -184 \\
\hline \multicolumn{3}{|c|}{ Korsmeyer-Peppas model } \\
\hline $\mathrm{k}$ & 0.084 & 0.116 \\
\hline $\mathrm{n}$ & 0.587 & 0.73 \\
\hline $\operatorname{adj}-R^{2}$ & 0.935 & 0.911 \\
\hline $\mathrm{F}$ & 302 & 226 \\
\hline AIC & -58 & -76 \\
\hline \multicolumn{3}{|l|}{ Weibull model } \\
\hline$a$ & 4.644 & 4.559 \\
\hline $\mathrm{b}$ & 0.525 & 0.461 \\
\hline $\mathrm{T}_{d}(\mathrm{~h})$ & 0.87 & 0.84 \\
\hline adj-R $R^{2}$ & 0.956 & 0.817 \\
\hline $\mathrm{F}$ & 368 & 77 \\
\hline $\mathrm{AIC}$ & -86 & -63 \\
\hline
\end{tabular}

Table 6.6: Adjusted parameters, the adjusted coefficient of determination, $F$ statistic, and AIC (Akaike Information Criteria) values for kinetic model fits of the release data for two types of nanovehicles. 
on their surface, and therefore the amount of agent dissolved by unit of time decreases. The first type of DOX vehicles presents good fit of the release data also in case of zero order kinetic model. This can be due to the slow rate of drug release during the first phase of the overall release process which results in the proportional relationship between the cumulative drug release and time. It is known that the pharmaceutical forms which are in good accordance with the zero order kinetics model are the ideal way of antitumor agent release to obtain a pharmacological action lasting a long period of time. It is obvious that for the second type of DOX carriers the calculated zero-order model fails to fit the release data (See Tab. 6.6). The other good data fit can be obtained with the Korsmeyer-Peppas model (the Power Law model) (Fig. 7.16). This approach is so general (although it lacks sufficient basis) that it is likely that it will fit any reasonable set of data. Concerning the $n$ value utilized to characterize the release, it can be estimated by fitting the initial part of the curve $(<0.6)$. It can be evaluated that the release mechanisms governing the drug release from first and the second type of DOX vehicles were diverse, as the $n$ value are equal to 0.59 and 0.73 , respectively. This means that they follow anomalous drug transport behaviour which is intermediate between Fickian diffusion and Case-II transport (mass transfer following a non-Fickian model).

The other model for which good fit parameters were obtained was the Weibull model (See Fig. 7.15). The Weibull equation (Eq. 7.3) can be rearranged to give:

$$
\log [-\ln (1-m)]=b \log \left(t-T_{i}\right)-\log a .
$$

From this equation a linear relation between the logarithm of the dissolved amount of drug vs. the logarithm of time is obtained. The parameter $m$, which expresses the accumulated fraction of the drug in solution at time $t$, will be in our case the accumulated concentration of the doxorubicin in the PBS solution. The shape parameter $b$ is determined from the slope of the fitted line and the scale parameter $a$ is evaluated from the ordinate value $(1 / a)$ at time $=1$. In some cases the scale parameter is substituted by the more instructive dissolution time, $T_{d}$, that is determined as $a=$ $\left(T_{d}\right)^{b}$ and is taken from the plot because the $t$ value corresponding to the ordinate $-\ln (1-m)$ is equal to 1 . From this fit the $a$ value equal to 4.64 and 4.56 for the first and the second type of DOX carriers were estimated, respectively. The time parameter, $\mathrm{T}_{d}$ calculated from the $a$ and the $b$ values is 0.87 (in hours) and 0.84 (in hours) for the first and the second DOX vehicles, respectively. They represent the time interval necessary to dissolve 

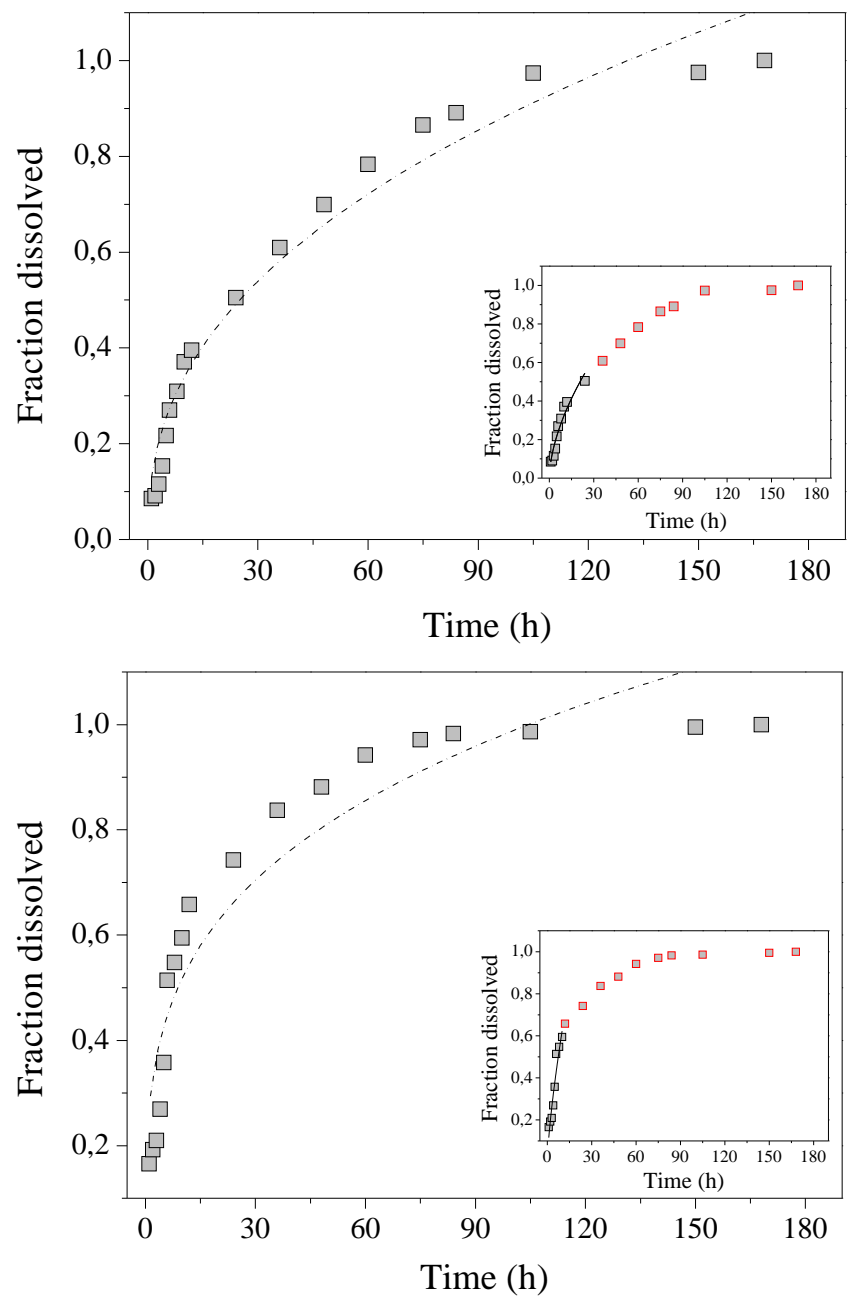

Figure 6.8: Doxorubicin release fraction from magnetic nanocomposites Top:the first type of DOX nanoparticulate carriers; Bottom: the second type of DOX vehicles. The dotted line in both graphics is the fit of the release data to Korsmeyer-Peppas model (the Power Law). Details - determination of the diffusional exponent which is indicative of the transport mechanism. 
$63.2 \%$ of the cytotoxic agent. A higher $T_{d}$ value indicates slower release rate, indicating that in our case the first nanosystem is characterized by a slower release process. Therefore, it may be concluded that the second type of DOX carriers gave faster release as compared with the first one. Concerning $b$ values, 0.52 and 0.46 for the first and the second type of DOX vehicles were obtained, respectively. These values of the shape parameter $(<1)$ indicate a parabolic curve with steeper initial slope which is coherent with the exponential. The in-vitro cytotoxic agent release profile from nanoparticles can be similar to that inside the body even though the rate is habitually faster in case of in-vivo conditions because of the existence of various enzymes and surfactants in biological environments. A PBS solution prepared as a dissolution medium imitates the $\mathrm{pH}$ and salt concentration in biological fluids. As published earlier (Kim \& Chu, 2000), when comparing DOX release processes in various $\mathrm{pH}$ conditions, the amine part of doxorubicin will stay in its unprotonated form (non-ionized) at $\mathrm{pH}$ 7.4. When the $\mathrm{pH}$ value decreases to 3.0 , the majority of amine groups will be ionized (Sturgeon \& Schulman, 1977), causing more release. Consequently, the existence of ionizable groups in agents will be a crucial factor for predicting their release profile in different $\mathrm{pH}$ conditions (See Fig. 6.9).

It is reasonable that the release of DOX from the gold surface is mainly due to diffusion into the culture medium. Of the many other mechanisms that have been proposed (matrix dissolution, swelling, vehicle erosion, action of external fields, ... ) none is applicable to our systems, considering the stability of the gold substrate. Concerning the breaking of bonds between DOX and the vehicle, we could only think of the so-called "environmentally responsive"systems, in as much as the charges of both the drug and the substrate are $\mathrm{pH}$-dependent in certain $\mathrm{pH}$ intervals. Specifically, DOX is positively charged only up to $\mathrm{pH}$ 8.3, whereas mobility data (See Figs. 4.4 and 4.6 in Chapter 4) shows that the substrates get their maximum negative charge at that $\mathrm{pH}$; hence, at the $\mathrm{pH}$ of the release experiments (7.4) the interaction is weakened by the lower average positive charge per DOX molecule, as compared to the adsorption stage (6-6.5, natural $\mathrm{pH})$. In addition, the ionic strength of the culture medium $(0.15 \mathrm{M})$ will partially screen the attractive interaction between DOX and nanoparticles. These factors favor diffusion as the only (passive) mechanism of release. Note also that the time required for release is well suited for antitumor treatment, since high plasmatic concentrations would be maintained for almost two days. 


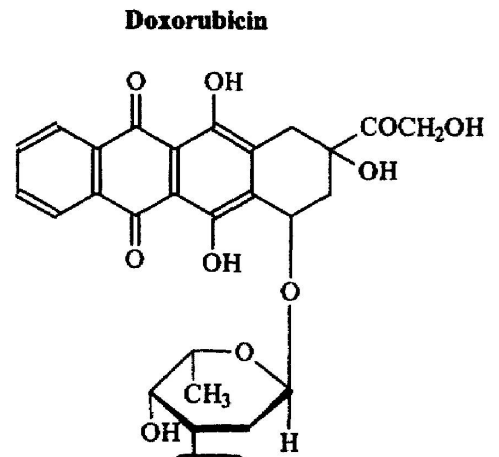

$\mathrm{NH}_{2}$

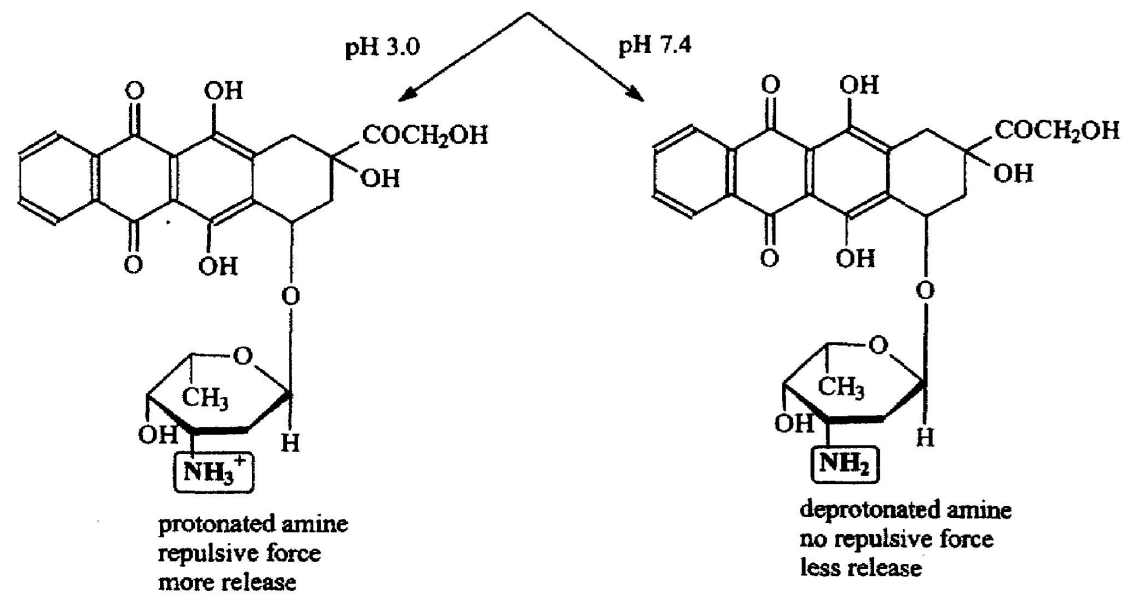

Figure 6.9: Ionic forms of doxorubicin in various $\mathrm{pH}$ conditions and its effect on the release profile (Kim \& $\mathrm{Chu}, 2000)$. 


\subsection{Confocal fluorescence microscopy.}

One more convincing proof/indication for the cellular uptake of doxorubicin delivered by the nanovehicles into tumor cells are the photos obtained by means of confocal fluorescence microscopy, like those presented in Figs. 7.17, 7.19 and 7.18. The partial planar structure of the tetracyclic ring in the cytotoxic agent molecule, makes it fluorescent at the wavelength of $533 \mathrm{~nm}$ upon laser excitation at $488 \mathrm{~nm}$. This fact permits to confirm the presence of the antitumor drug on the nanostructures, and finally its in vitro release in the chosen tumor cell lines. The results obtained verify that doxorubicin is released in the nuclei of cancer cells, as can be observed in Figures 7.17, 7.19 and 7.18: the red colour fluorescence typical of anthracycline antibiotic is noticed as either free doxorubicin or DOX adsorbed onto the nanocomposites is present in the culture medium. The presence of fluororescence is restricted to the nuclei in all cases and it confirms the nanostructures gain entrance to the cell nuclei and are able to move from in the cytoplasm, after 2 hours contact. The results were obtained for both tumor cell lines (liver tumor cells PLC-PRF-5 (Fig. 7.18) and colon tumor cells DLD-1, Figs. 7.17 and 7.19)) and both types of DOX carriers (compare Fig. 7.17 and Fig. 7.19).

\subsection{Cell viability studies}

The antitumor action of the designed vehicles will be evaluated by studying tumor cell viability when in contact with the particles. This was done by the sulphorhodamine B method as described by (Valenzuela et al., 1995). The study was performed only in the case of the second type of DOX carriers as previous experiments showed the adequacy of this system for drug delivery. The second DOX nanovehicles presented better stability in the culture medium suggesting their favourable in vitro behaviour, as also confirmed by data presented in Tab. 6.1 containing hydrodynamic sizes of final nanovehicles from the second design. Moreover, the drug loading capacity of the second type of DOX nanocarriers is quite bigger than that of the first design, what permits us to predict that this one will cause more cytotoxic effect on the tumor cells. Figs. 7.21 and 7.20 show the viability assays of the two cell lines at three dilutions of nanoparticles, compared to the free DOX treatment at comparative dilutions (starting from $0.3 \mathrm{mM}$ solutions), and the (untreated) control cells. Note first of all that bare particles significantly affect the viability only at the highest 


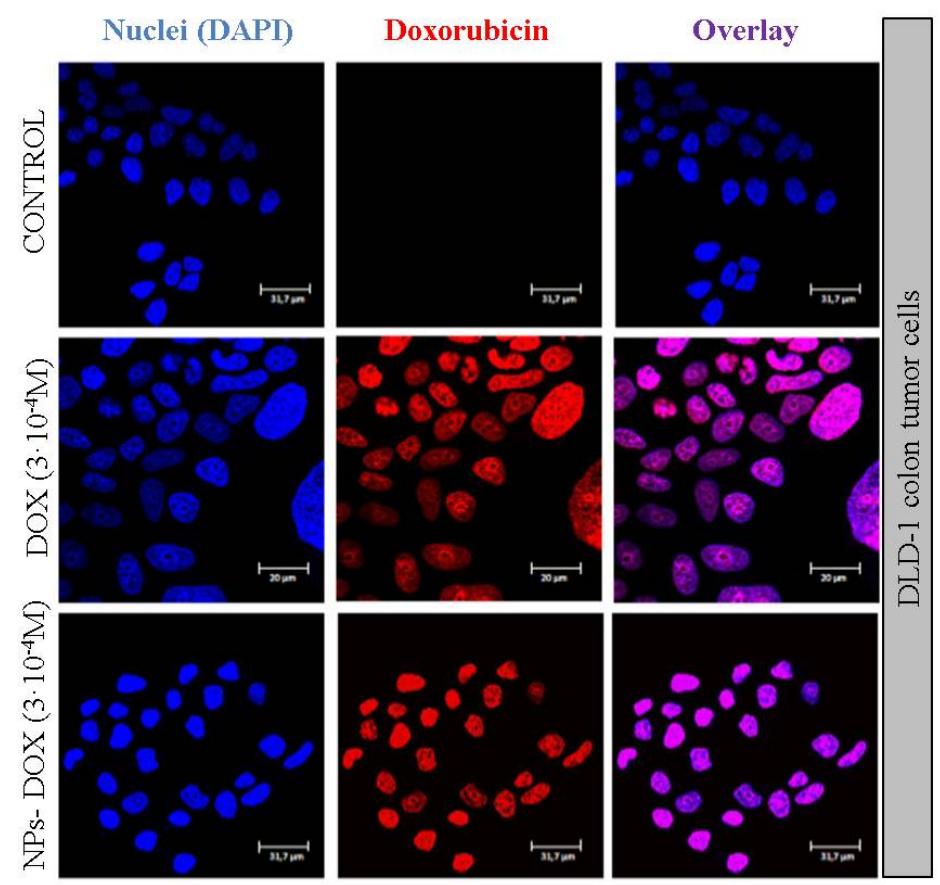

Figure 6.10: Confocal images of colon tumor cells (DLD-1) untreated, and treated with gold-coated magnetic nanoparticles from the first nanoparticulate system. The blue color indicates the staining for nuclear imaging with DAPIDNA in confocal microscopy. Top row: control without either nanoparticles or drug; middle and bottom rows: with nanoparticles loaded with the indicated concentrations of DOX after 2 hours. Particle concentration: $1.8 \mathrm{mg} / \mathrm{mL}$. 


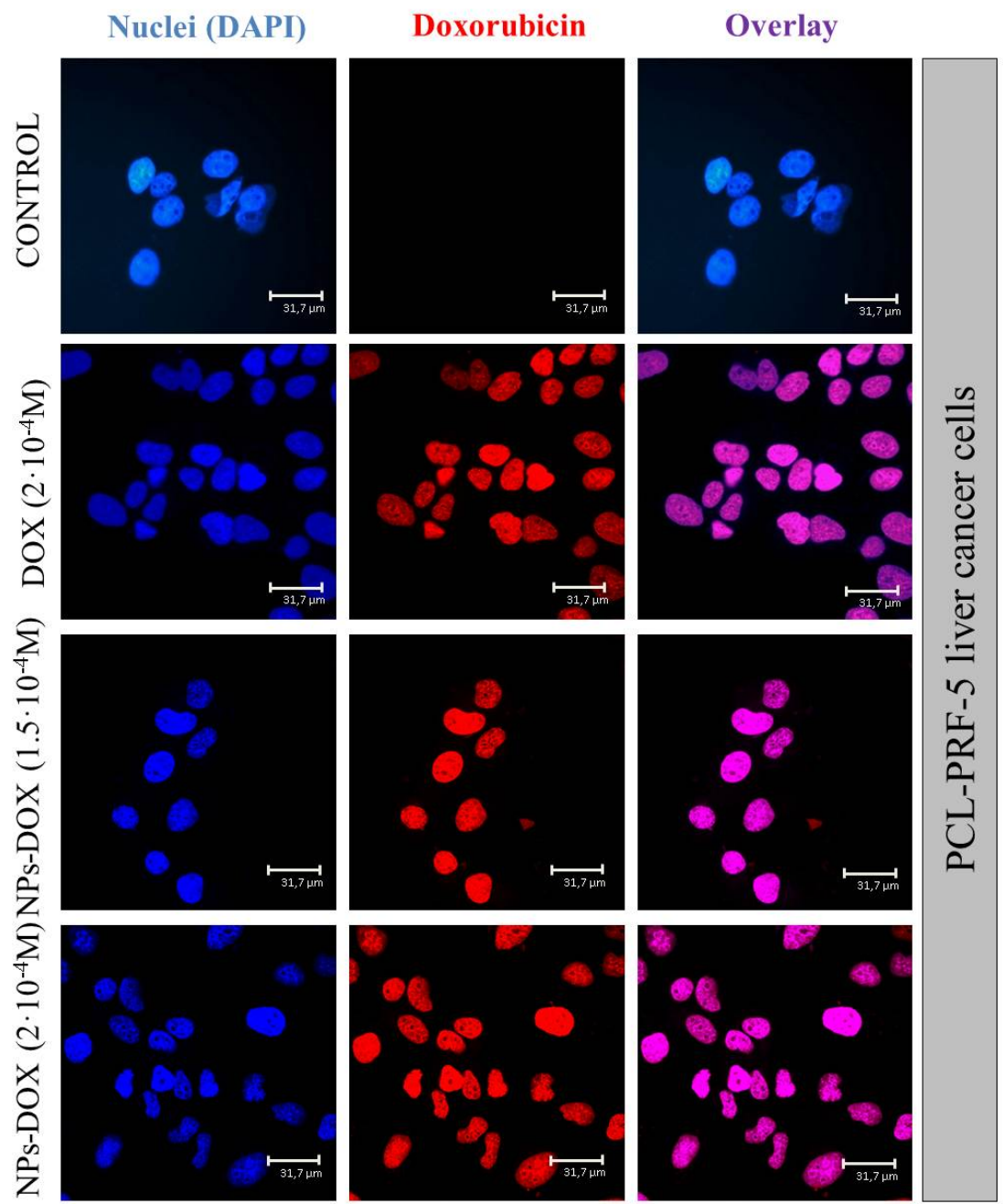

Figure 6.11: Confocal images of liver tumor cells (PLC-PRF-5) untreated, and treated with gold-coated magnetic nanoparticles from the second nanoparticulate system. The blue color indicates the staining for nuclear imaging with DAPI-DNA in confocal microscopy. Top row: control without either nanoparticles or drug; middle and bottom rows: with nanoparticles loaded with the indicated concentrations of DOX after 2 hours. Particle concentration: $1.25 \mathrm{mg} / \mathrm{mL}$. 


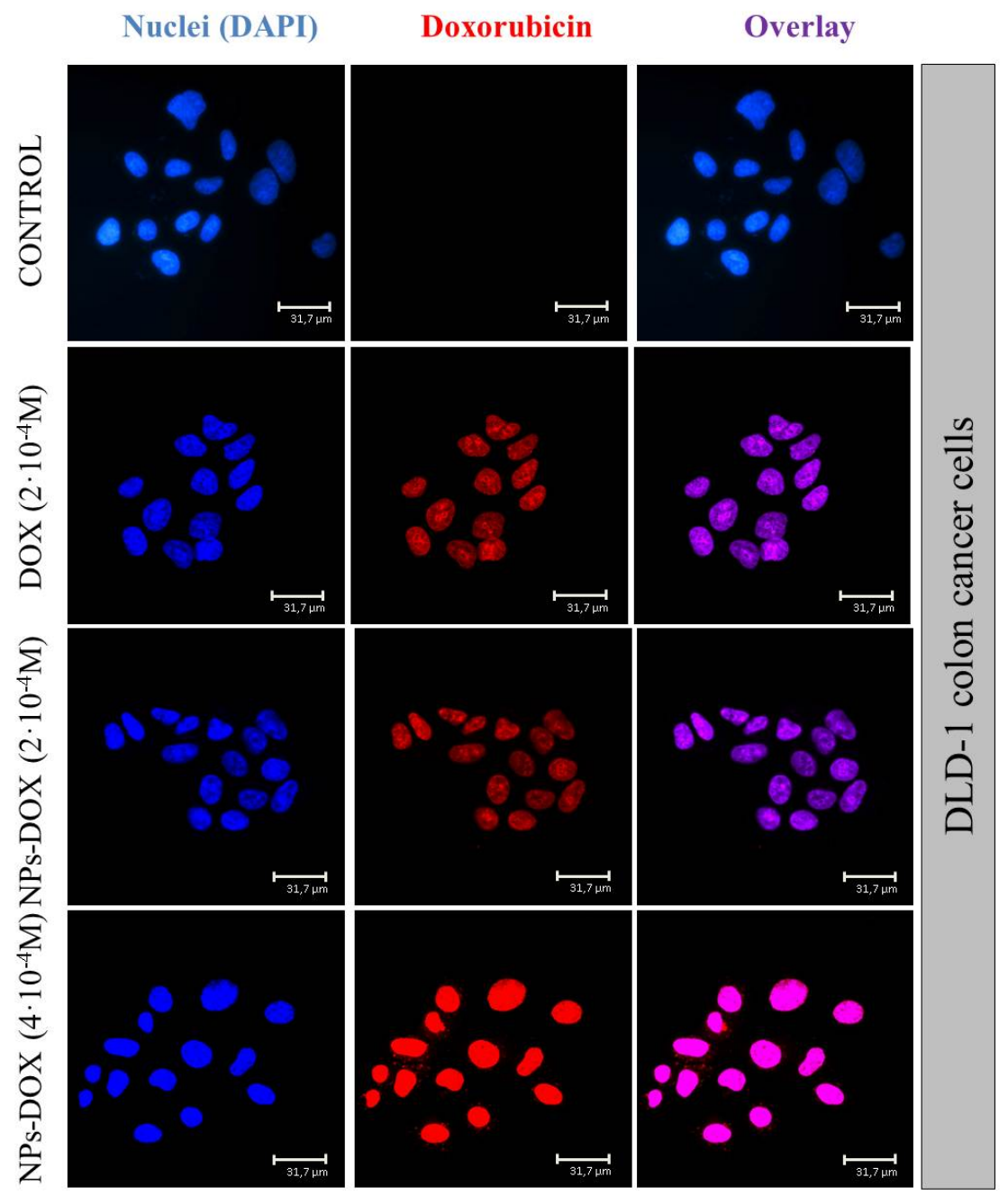

Figure 6.12: Same as Fig. 7.18, but for colon tumor cells (DLD-1). Solutions of free DOX and DOX-loaded nanoparticles were incubated for $2 \mathrm{~h}$ in presence of DLD-1 colon cancer cells. Cell nuclei were counterstained with DAPI (blue). Particle concentration: $1.25 \mathrm{mg} / \mathrm{mL}$. 

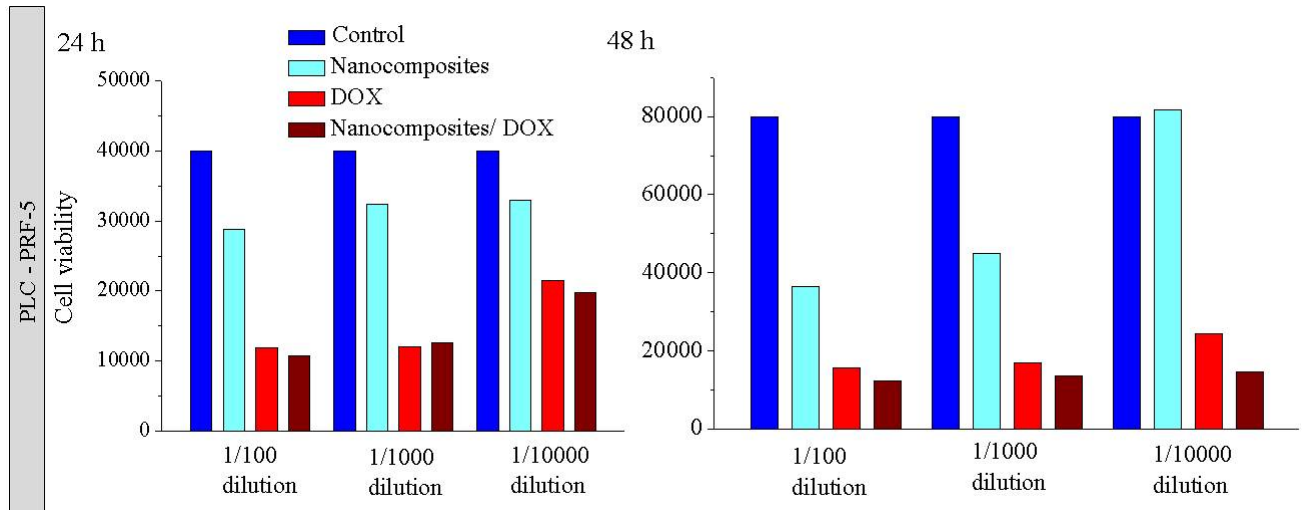

Figure 6.13: Cell viability assays for PLC-PRF-5 liver cancer cells treated for 24 and $48 \mathrm{~h}$, with nanocomposites, doxorubicin in different amounts, and doxorubicin adsorbed onto nanocomposites. Particle concentration in the nondiluted suspensions: $1.25 \mathrm{mg} / \mathrm{mL}$.

concentration and longest contact times $(48 \mathrm{~h})$. In the case of liver tumor cells (Fig. 7.20), it can be appreciated that the cytotoxic effect produced by free DOX is comparable to that of drug-loaded particles, and only for the highest dilution and after $48 \mathrm{~h}$ there appears some increased efficiency of the particles compared to free DOX in solution. The effect is much more noticeable with DLD-1 colorectal cells (Fig. 7.21): while for liver cells the viability is $31 \%$ with DOX and $20 \%$ with nanoparticles, the corresponding data for DLD-1 cells are $51 \%$ and $22 \%$, respectively. These data refer to the optimum conditions, that is, $48 \mathrm{~h}$ contact and $1 / 10000$ dilution. We can conclude that, because of the larger difference between DOX solution and DOX-loaded nanoparticles, these might be a better option in colorectal tumors.

As a final point, clear field microscope pictures like the ones depicted in Fig. 7.22 show the viability after 48 hours for the control DLD-1 cells (Fig. 7.22 A), the same cells treated with free DOX (Fig. 7.22 B), goldcoated maghemite/silica nanoparticles (Fig. 7.22 C), and with DOX-loaded nanoparticles (Fig. 7.22 D). Contrasting the four photos, it can be easily noticed that the cellular viability is significantly reduced after contact with the DOX-loaded nanocomposites compared to the cell death caused by the free antitumor drug. Finally, it is worth mentioning that the nanostructures alone caused very little toxicity on the DLD-1 tumor cells, most likely because of stress derived from the endocytic pathway overstimulation. 

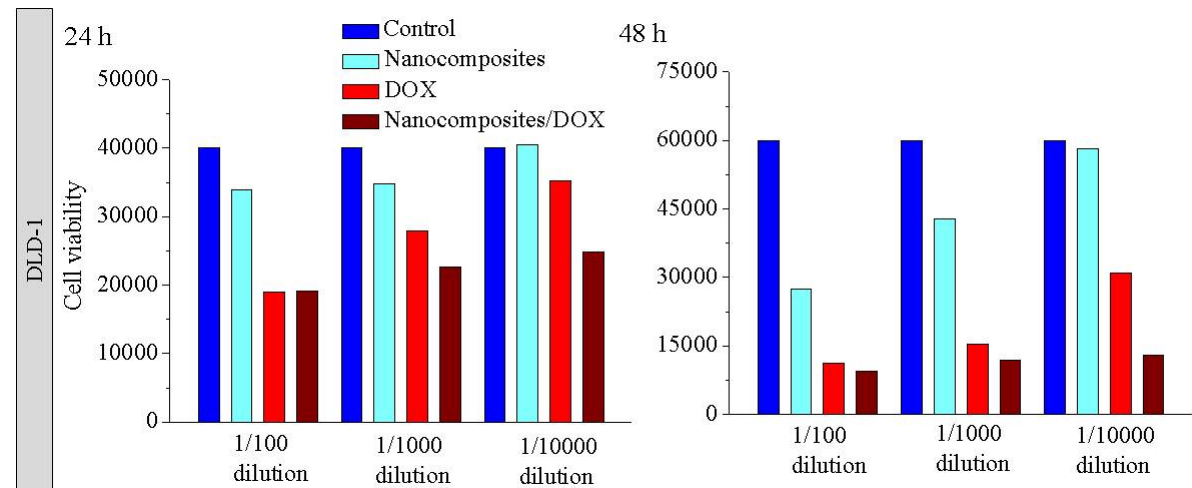

Figure 6.14: Same as Fig. 7.20, but for DLD-1 colorectal cancer cells.

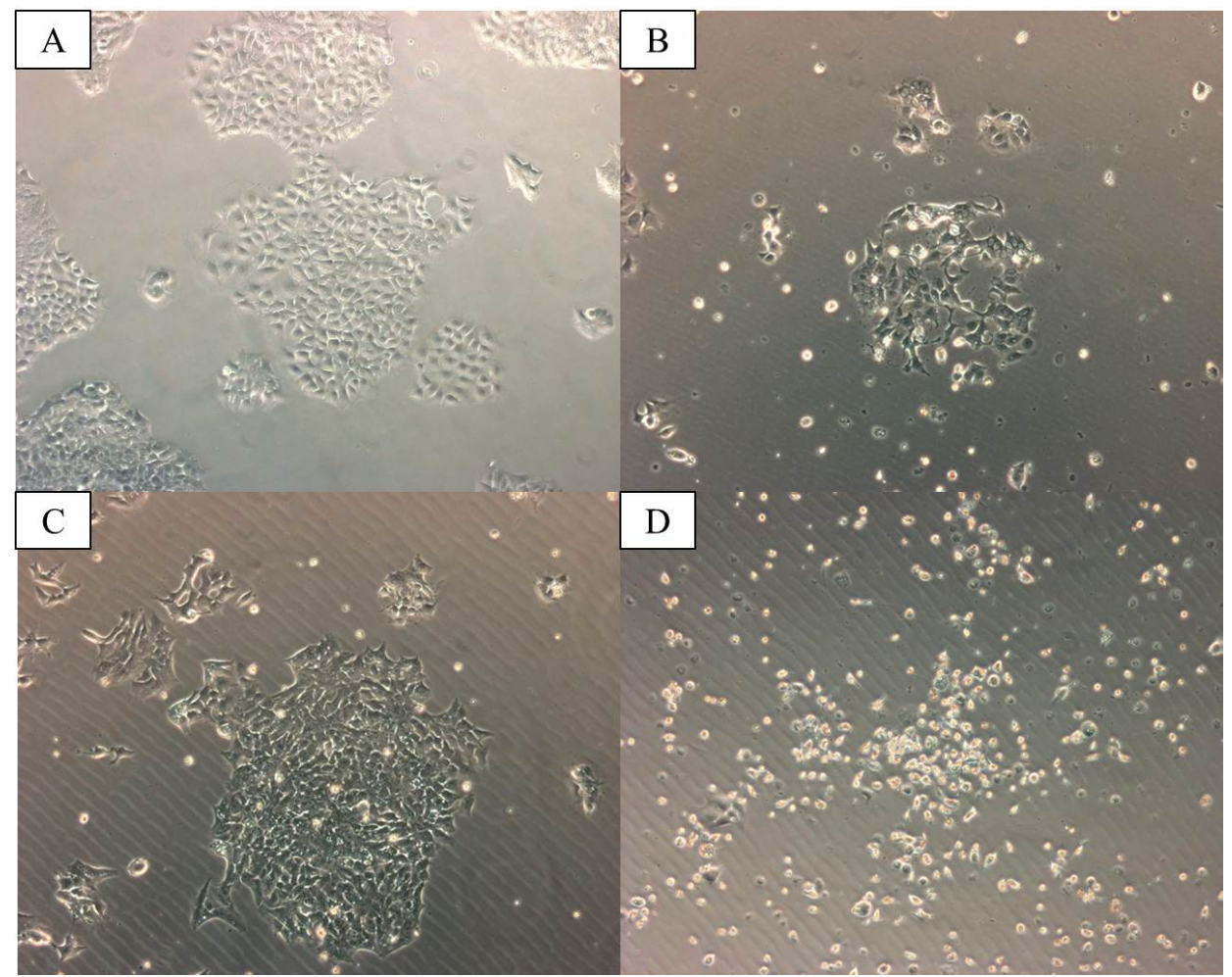

Figure 6.15: Clear field microscope images of DLD-1 colon tumor cell. A: control; B: in contact with free DOX $(0.3 \mathrm{mM})$; C: in contact with nanoparticles; D: with doxorubicin $(0.3 \mathrm{mM})$ adsorbed onto nanocomposites. All photos were taken after $48 \mathrm{~h}$ contact for the second nanoparticulate system. 


\subsection{Conclusions}

When comparing two nanoparticulate systems as DOX vehicles, it can be observed that the maximum adsorption density is almost two times higher in the case of the second nanocomposites design, and the dissimilarities, in this regard, between the two designed systems is considered to be very determinant. The second approach has the benefit of a more compressed and well defined final gold shell, that will finally make these nanocomposites more adequate for work with living tissues, and with more predictable manner of acting after antitumor drug system administration. In addition, there are differences in the relase of the payload of doxorubicin. As shown in Fig. 7.14, the first nanosystem is able to release only $46 \%$ of its payload, whereas the second goes up to $70 \%$. The in-vitro release confirms this, as shown by confocal microscopy in Figs. 7.17, 7.19 and 7.18, where photos of tumor cells treated with nanostructures of both types can be observed. 
Chapter 7

\section{Resumen}





\subsection{Introducción. Objetivos}

La lucha contra el cáncer se ha convertido en objetivo esencial de la actividad científica y de los sistemas de salud en todos los países desarrollados. Se han propuesto diferentes aproximaciones al problema, pero las más recientes se basan en el diseño de sistemas de liberación capaces de mejorar la selectividad y especificidad de acción de los principios activos. Esto se aplica especialmente al caso de los antitumorales debido a la variedad y gravedad de efectos adversos que produce su distribución sistémica (Kim et al., 2012; Nakamura et al., 2012; Senter \& Sievers, 2012). Las contribuciones de investigaciones, como la que se presenta en este trabajo, se centran por ello, en el diseño de nanoestructuras enfocadas al transporte del agente terapéutico hasta su lugar de acción y su liberación de manera controlada.

Uno de los diseños se centra en el empleo de sistemas de transporte y liberación de fármacos basados en nanopartículas. Se considera que el tamaño nanométrico favorecerá la entrada del fármaco en la célula objetivo. Tambin, la elevada superficie específica permitirá una gran carga de principio activo as como la irritación local en el sitio de administración (normalmente inyección intravenosa) será muy limitada (Thassu et al., 2007). En concreto, proponemos el empleo de sistemas magnéticos de liberación. La idea de emplear partículas magnéticas como método de conducir el fármaco y favorecer su acumulación nació en los años 70 (Widder et al., 1979; Senyei et al., 1978). Sin embargo, sólo recientemente se ha profundizado en las aplicaciónes biomédicas de las nanopartículas magnéticas, en aspectos tales como imagen por resonancia magnética (Schlorf et al., 2011), hipertermia, o liberación de fármacos y genes (Gupta et al., 2007; Viota et al., 2011). El material más frecuentemente usado con estos fines se basa en nanopartículas superparamagnéticas de óxido de hierro, o SPions, normalmente como núcleos de estructuras funcionalizadas para aumentar su biocompatibilidad, servir de soporte al depósito de fármaco o dotarlas de receptores específicos para células tumorales concretas (Pankhurst et al., 2003).

La experiencia de nuestro grupo en la investigación de sistemas dispersos formados por micro- o nanopartículas dispersas en medio acuoso ha abierto muchas posibilidades en la búsqueda de aplicaciónes en campos como el biomédico. En concreto, hemos trabajado en el diseño de sistemas de transporte y liberación de fármacos, incluyendo sistemas magnéticos. (Duran et al., 2008; Rudzka et al., 2012; Viota et al., 2011, 2013). En esta tesis, esta línea se amplia para desarrollar los siguientes objetivos: 
- Síntesis y caracterización de las nanopartículas magnéticas. Se usa maghemita como núcleo magnético. Se caracterizan las partículas obtenidas en cuanto a su forma, tamaño, composición y propiedades magnéticas.

- Aplicación de un recubrimiento de sílice. Se ha comprobado que la sílice es un excelente material para aplicaciónes biomédicas, atendiendo a su buena biocompatibilidad (Arruebo et al., 2006; Barbé et al., 2004; Slowing et al., 2007, 2008; Zhao et al., 2005).

- Recubrimiento como capa de oro. Se hará uso de las grandes posibilidades que ofrece el oro en aspectos diagnósticos y terapéuticos. Seguimos dos rutas para alcanzar este objetivo. Una esta basada en el empleo de capas sucesivas de polielectrolito (catiónico - aniónico -catiónico, sistema capa a capa o LbL; polielectrolitos: PDADMAC y PSS, respectivamente). La segunda es la aplicación de una capa de APTMS (3-aminopropil-trimetoxisilano).

- Formación de complejos maghemita/oro. Se realiza mediante métodos de nucleación de nanopartículas de oro sobre la capa base de sílice, y extensión de una capa casi-continua de este metal mediante sucesivas nucleaciones.

- Transporte de fármaco. Es el verdadero núcleo del trabajo: se emplearán las nanoestructuras descritas para la adsorción y liberación de doxorrubicina (DOX). DOX es un fármaco catiónico de uso extendido y muy efectivo en el tratamiento de diferentes tumores sólidos, incluyendo linforma, sarcoma de Kaposi, osteoesarcoma, etc.

- Imagen por resonancia magnetica (MRI). Se analiza el empleo de estas nanopartículas como agentes de contraste en imagen por resonancia (MRI): nuestras partículas podrían idealmente combinar capacidad de liberación de DOX y posible empleo en diagnostico MRI.

\subsection{Síntesis y caracterización de núcleos magnéticos}

\subsubsection{Estrategia de síntesis}

Existe una amplia variedad de métodos de preparación de coloides magnéticos (Li et al., 2006), cada cual tiene sus ventajas e inconvenientes. Puede decirse que ninguno es completamente satisfactorio y de validez general. Sin embargo, está claro que los más frecuentemente utilizados y fiables son los procedimientos de precipitación o coprecipitación en medio líquido, partiendo de disoluciones de sales de los iones magnéticos de interés. El método usado 


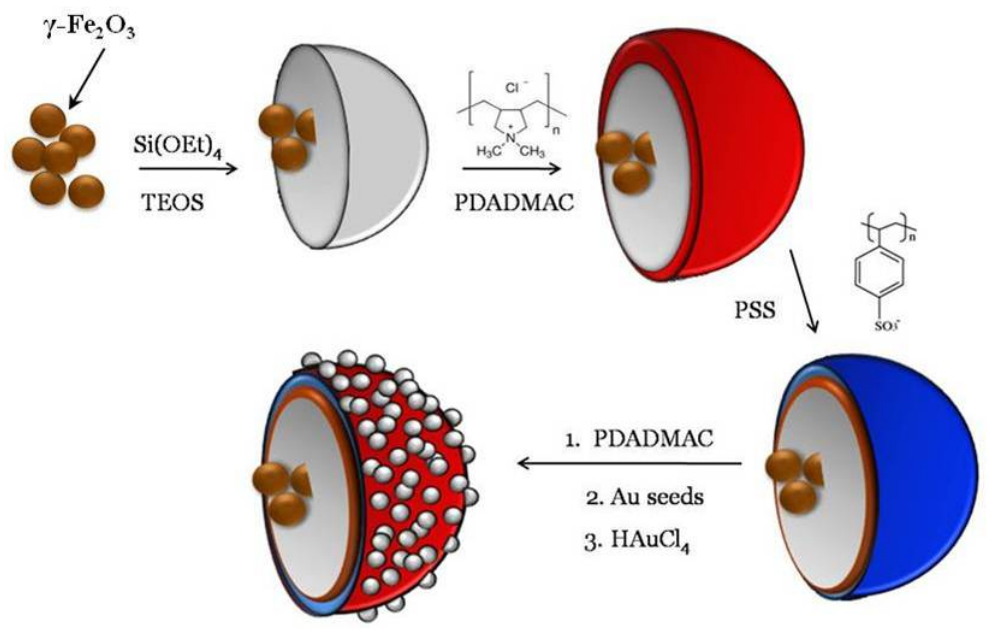

Figure 7.1: Ilustración del metodo I.

en este trabajo es de este tipo. El punto de partida es la reacción de Massart (Massart, 1981), en la que se comienza por preparar nanomagnetita que posteriormente, se somete a un proceso de oxidación. Este consiste en añadir a las nanopartículas de magnetita resultantes una disolución $2 \mathrm{M}$ de $\mathrm{HNO}_{3}$ y $0.33 \mathrm{M} \mathrm{Fe}\left(\mathrm{NO}_{3}\right)$ y agitar a $90^{\circ} \mathrm{C}$.

Para el recubrimiento de sílice se siguió el procedimiento de (Salgueiriño Maceira et al., 2006), modificación del método clásico de Stöber (Stöber et al., 1968). Estas partículas mixtas fueron a su vez empleadas para el recubrimiento con oro, para lo cual se siguieron dos procedimientos:

Método I El punto de partida es la síntesis de nanopartículas de oro por el procedimiento de reducción con citrato sódico (Enüstün \& Turkevich, 1963). Posteriormente, las partículas de maghemita/sílice se recubren con tres capas sucesivas de polielectrolito (PDADMAC-PSS-PDADMAC) (Salgueiriño Maceira et al., 2006; Schmidt \& Thews, 1995) y estas nanopartículas se añaden a una suspensión de nanopartículas de oro. La etapa de recubrimiento final consiste en crecer oro sobre las partículas que son portadoras de semillas de oro. La Fig. 7.1 muestra las partículas obtenidas.

Metodo II Se parte de nuevo de maghemita recubierta de sílice. El siguiente paso es el tratamiento con APTMS (Pham et al., 2002). Con ello, las partículas adquieren el sustrato de carga positiva necesario para fijar las nanopartículas de oro (obtenidas en este caso por reducción de disoluciones 


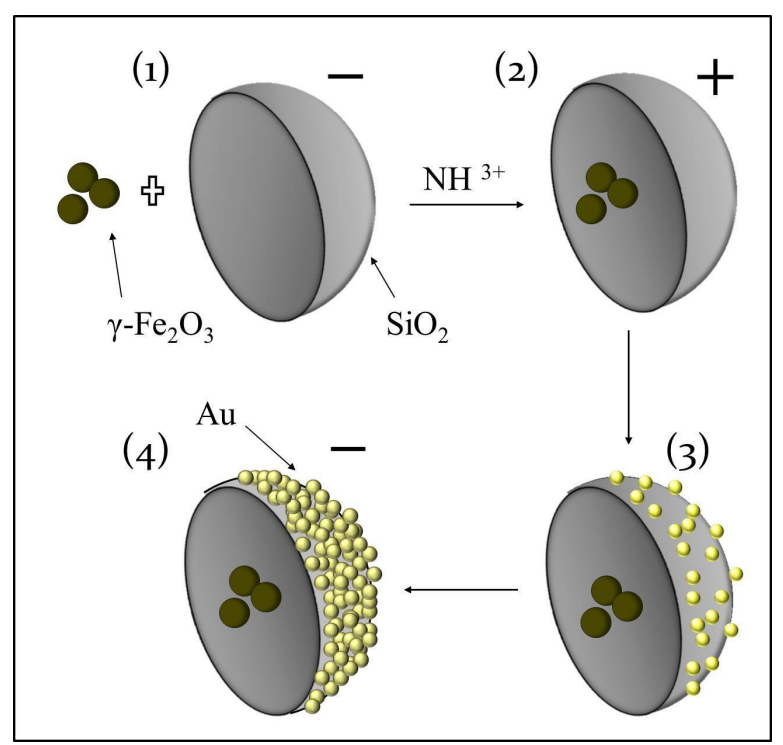

Figure 7.2: Representación del metodo II. 1) Recubrimiento con sílice; 2) tratamiento con APTMS; 3) Deposición de semillas de oro; 4) Crecimiento de la capa final.

de ácido cloroaúrico con $\mathrm{NaBH}_{4}$ ) (Busbee et al., 2003). Trás la deposición del oro, se procedió a una etapa de recubrimiento final, añadiendo los núcleos de maghemite/sílice /nano-Au a una disolución con ácido cloroaúrico y fijándolas con formaldehído (Fig. 7.2).

\subsubsection{Estudio morfológico}

La Fig. 7.3 ilustra las partículas de maghemita obtenidas. Nótese que son aproximadamente esféricas y muy monodispersas, con un tamaño próximo a los $15 \mathrm{~nm}$. Este resultado coincide con el obtenido mediante scattering estático y dinámico de luz láser (Malvern Mastersizer 2000 y Malvern PCS 3700, Malvern Instruments, UK).

Se llevó a cabo un estudio similar con partículas recubiertas de sílice. La Fig. 7.4 es un ejemplo. Es interesante destacar que cada partícula compuesta contiene varios núcleos de maghemita, y que su diámetro final es de $202 \pm 14$ nm, según los datos de scattering dinámico de luz. 

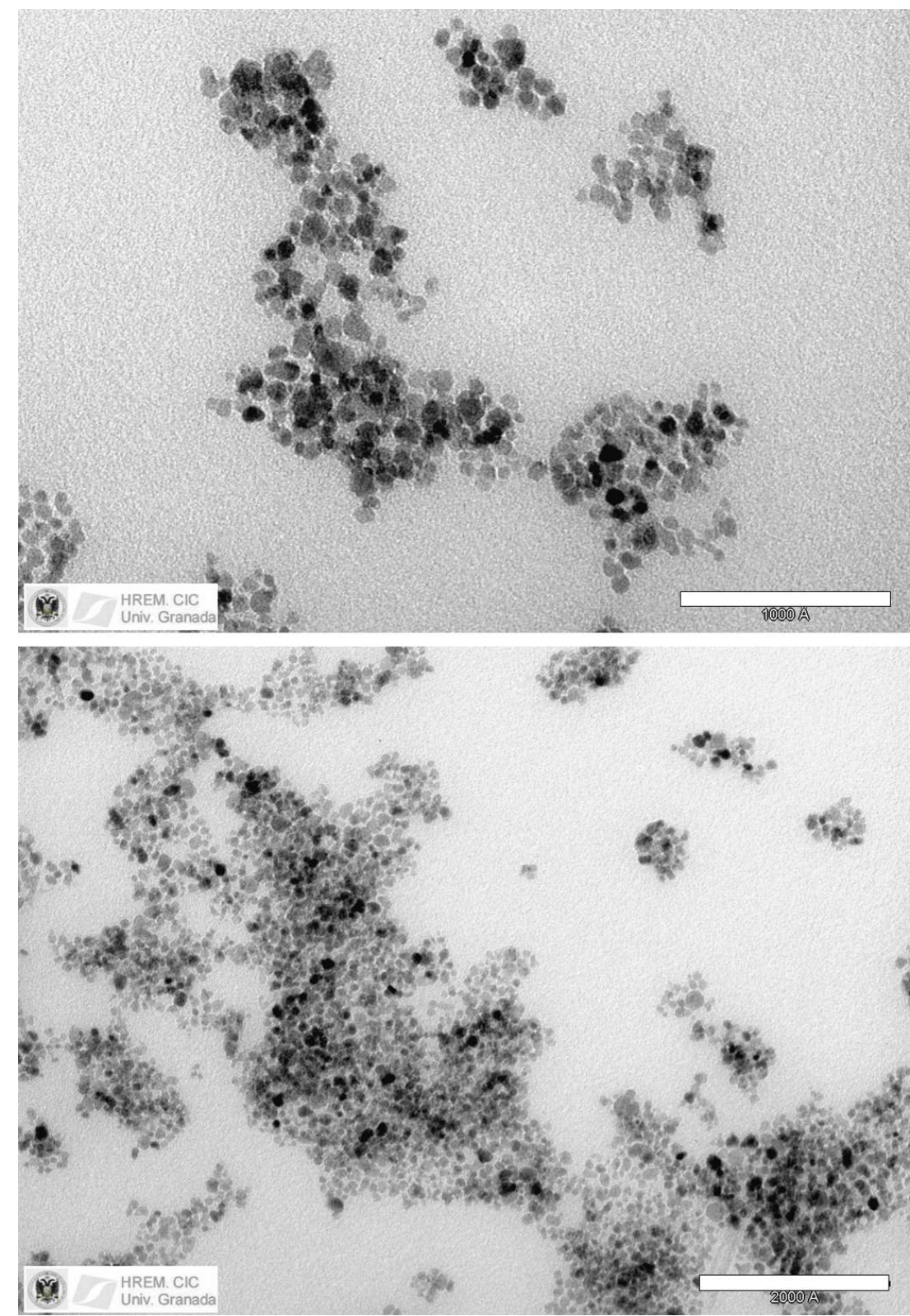

Figure 7.3: Fotografías TEM de nanopartículas de maghemita. Barras: 100 $\mathrm{nm}$ (superior), $200 \mathrm{~nm}$ (inferior). 


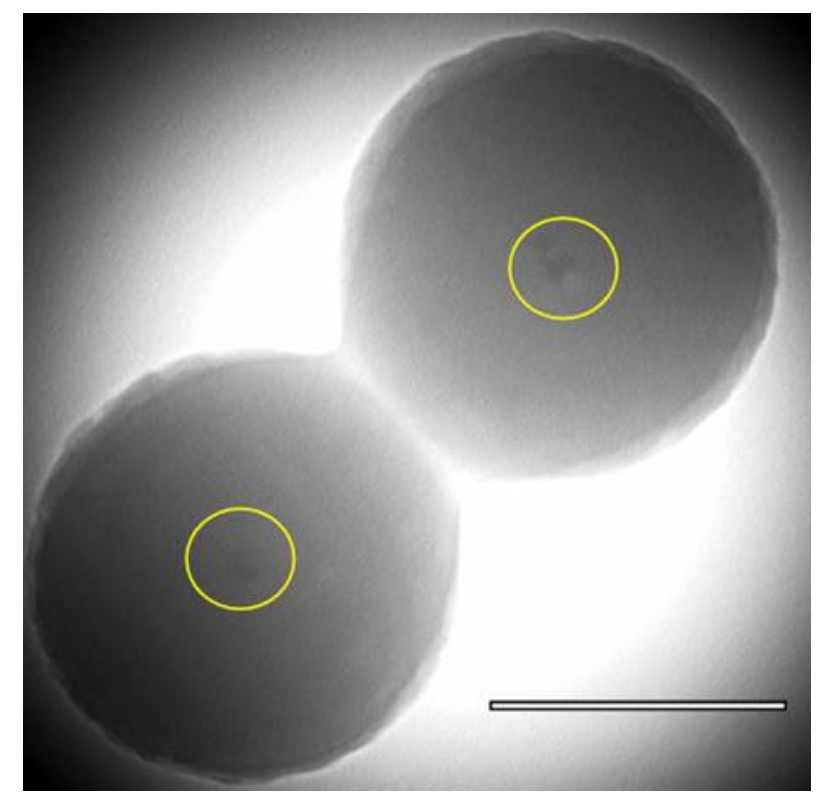

Figure 7.4: Partículas de sílice-maghemita vistas en microscopía TEM. Barra de $200 \mathrm{~nm}$. 


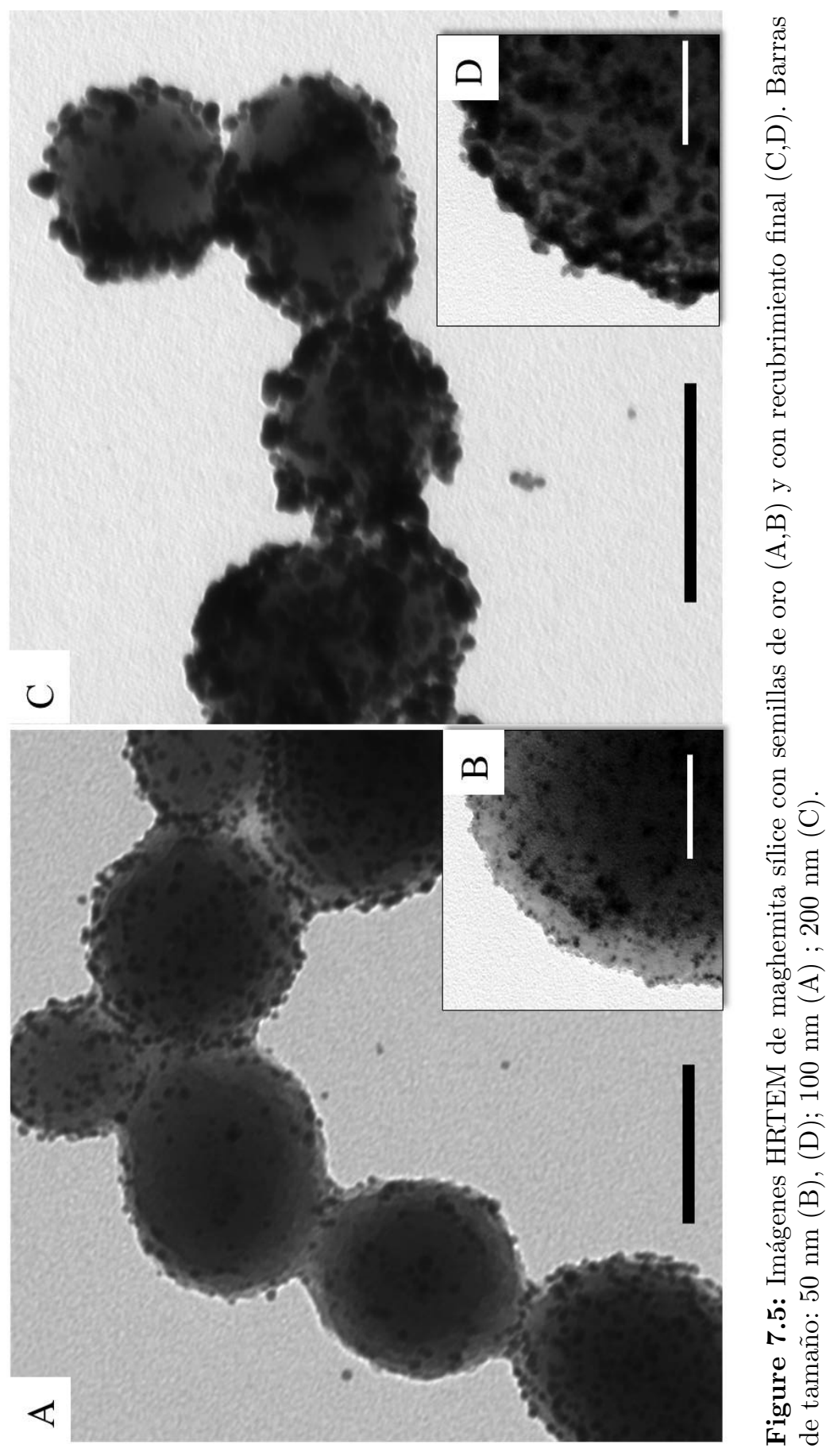


La Fig. 3.6 ilustra por su parte la geometría de las nanopartículas de oro, con tamaño en torno a los $10 \mathrm{~nm}$. Finalmente, mostramos en Fig. 7.5 imágenes de microscopía electrónica de alta resolución de las semillas $(\mathrm{A}, \mathrm{B})$ y la capa de oro $(\mathrm{C}, \mathrm{D})$ obtenidas con el método II.

\subsection{Propiedades Electrocinéticas}

En este apartado, mostramos como es posible sacar partido de la enorme sensibilidad de los fenómenos electrocinéticos, en concreto, de la electroforesis donde incluso pequeños cambios en la estructura superficial pueden significar variaciones en la distribución de carga en la superficie. Sin entrar en detalles en este resumen, baste recordar que en presencia de un campo eléctrico externo las partículas se mueven con una velocidad, la velocidad electroforética $\mathbf{v}_{e}$. Esta velocidad es proporcional al campo, siendo la constante de proporcionalidad la movilidad electroforética, $u_{e}$ :

$$
\mathbf{v}_{e}=u_{e} \mathbf{E},
$$

La expresión más simple de la movilidad en función del potencial electrocinético o potencial zeta, $\zeta$, es la de Helmholtz-Smoluchowski:

$$
\mathbf{v}_{e}=u_{e} \mathbf{E}=\frac{\varepsilon_{r s} \varepsilon_{0} \zeta}{\eta} \mathbf{E} .
$$

Las determinaciones de movilidad electroforética se llevaron a cabo en un dispositivo Zetasizer Nano-ZS (Malvern Instruments, U.K.), en suspensiones diluidas (fracciones de volumen de sólidos del orden de $10^{-4}$ ). Si se necesita, el potencial zeta se calcula a partir de la movilidad usando la teoría de O'Brien y White (O'Brien \& White, 1978).

\subsubsection{Resultados: diseño I}

La Fig. 4.2 muestra, en primer lugar, la valoración de potencial zeta de las partículas de maghemita, que manifiesta un punto isoeléctrico en torno a pH 7.5. Este resultado concuerda con los datos de otros autores que han trabajado con ferrofluidos basados en estas partículas (Halbreich et al., 1997). La presencia de la capa de sílice, por otro lado, cambia claramente la tendencia $\zeta \mathrm{pH}$, de modo que las partículas compuestas se comportan electrocinéticamente como la sílice (Rudzka et al., 2012), confirmando así la correcta cobertura del sustrato magnético. 


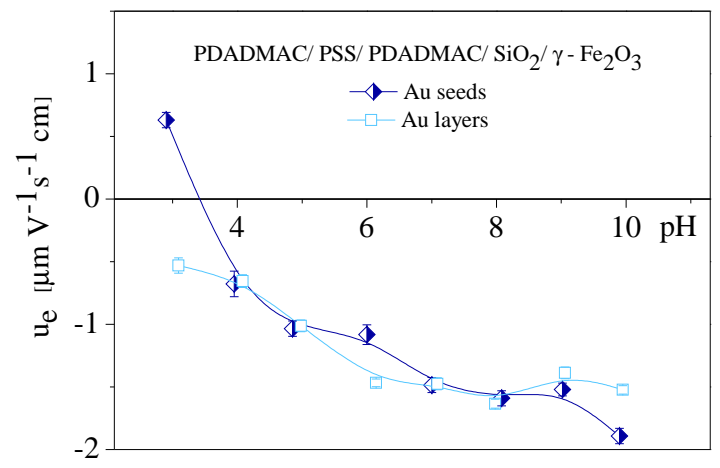

Figure 7.6: Comparación del efecto del $\mathrm{pH}$ sobre la movilidad de nanopartículas de maghemita/sílice con oro depositado.

Las determinaciones de movilidad nos ayudan también a detectar el avance de las sucesivas capas de polielectrolito en el método I de síntesis (Fig. 7.7). Nótese como el método LbL es una excelente técnica de modificación de superficies basada en el control de las interacciones electrostáticas entre capas sucesivas. Es también destacable el hecho de que, de acuerdo con las teorías sobre la electroforesis de partículas blandas, la movilidad no llega a anularse ni siquiera a concentraciones muy elevadas de iones (Ohshima, 2002). El efecto del recubrimiento de oro es igualmente detectable (Fig. $7.6)$.

\subsubsection{Resultados: diseño II}

El segundo es bastante diferente. En la Fig. 7.8 está claro que el polielectrolito catiónico APTMS se puede emplear para funcionalizar las esferas de sílice magnética, haciéndolas claramente positivas en un amplio intervalo de $\mathrm{pH}$ y adecuadas para el depósito de oro (Fig. 7.9). El cambio de punto isoeléctrico es una excelente indicación de la adsorción de semillas de oro. 


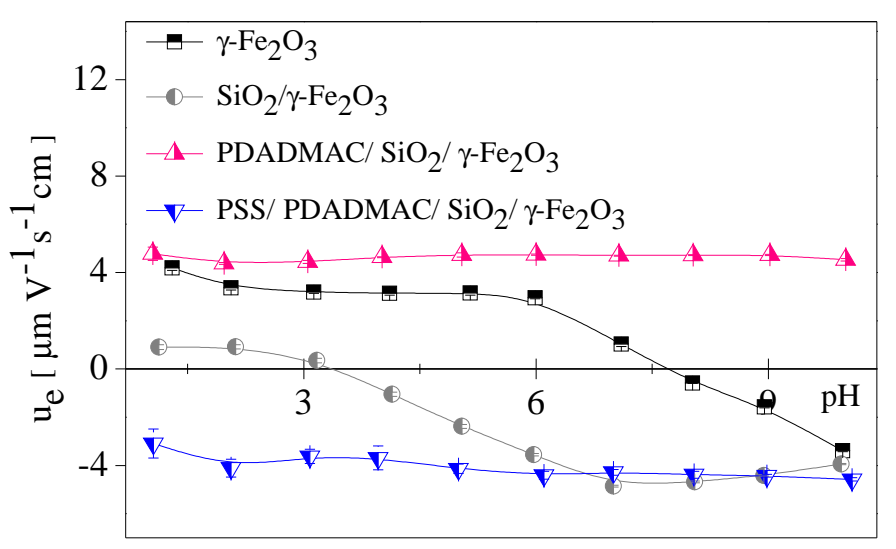

(a) $u_{e}$ en función del $\mathrm{pH}$ en $5 \mathrm{mM} \mathrm{KNO}_{3}$.

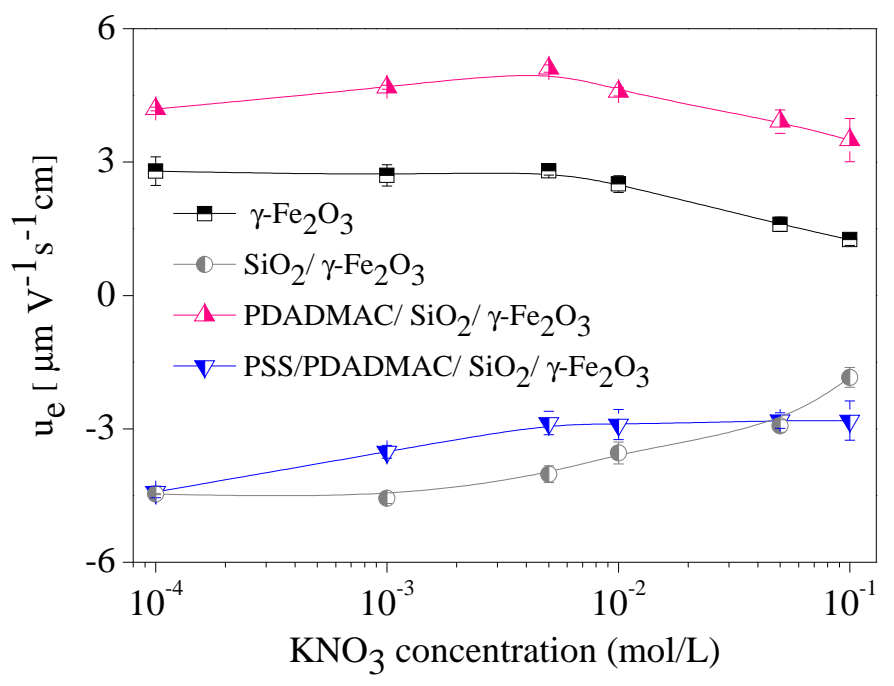

(b) $u_{e}$ en función de la fuerza iónica a $\mathrm{pH}$ constante.

Figure 7.7: Movilidad electroforética de maghemita y maghemita/sílice, antes y después del recubrimiento con polielectrolitos. 


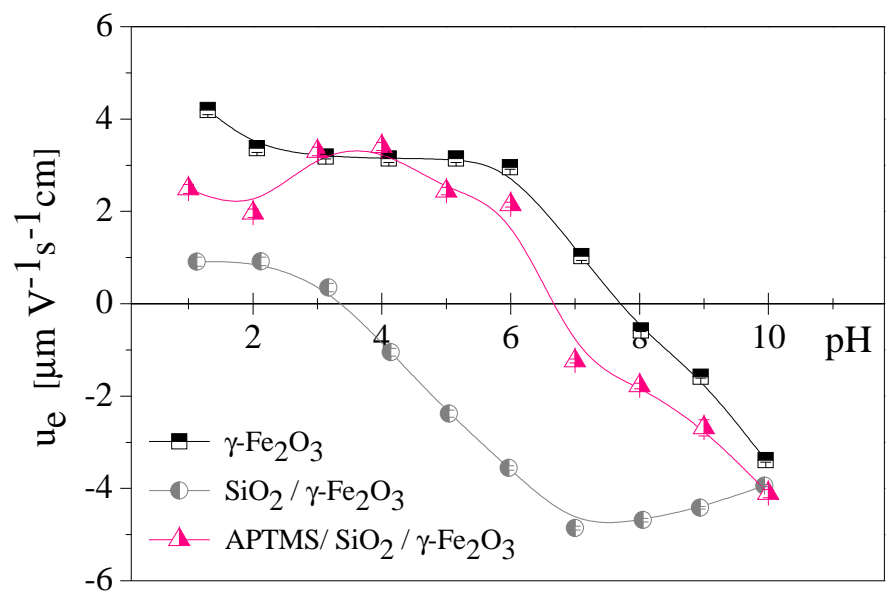

(a) $u_{e}$ en función del $\mathrm{pH}$.

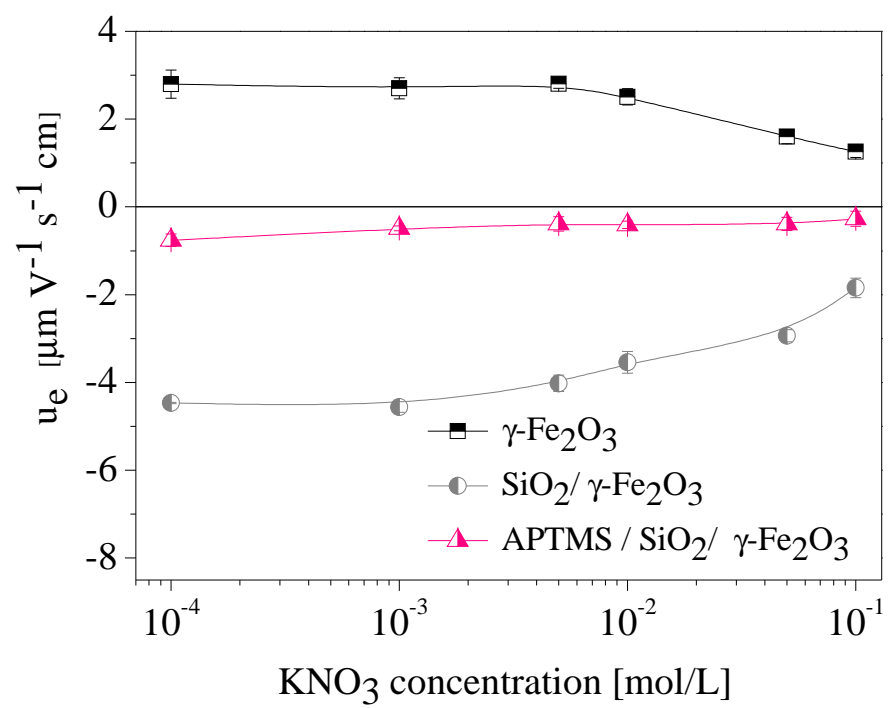

(b) $u_{e}$ en función de la fuerza iónica.

Figure 7.8: Movilidad de $\gamma-\mathrm{Fe}_{2} \mathrm{O}_{3}$, maghemita/sílice y maghemita/sílice con capa de APTMS depositada. 


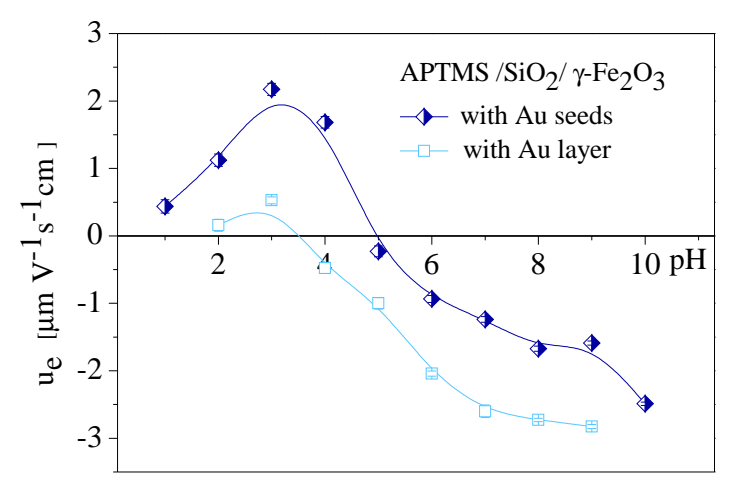

Figure 7.9: Comportamiento electroforético después del recubrimiento con oro, para el diseño II.

\subsection{Capacidad de adsorción de doxorrubicina}

\subsubsection{Aspectos generales}

Como se ha mencionado anteriormente, es esencial que el fármaco antitumoral alcance las células afectadas en concentraciones suficientes, mientras los efectos secundarios se reducen al máximo, es decir, con poco efecto en células no tumorales (Flórez, 2008). Queremos comprobar en este apartado si, en efecto, los vehículos diseñados son capaces de adsorber una carga suficiente de fármaco y liberarla después, tal como se ha descrito para otros vehículos magnéticos (Arias, 2008, 2011). Se usará doxorrubicina, como se ha mencionado (Fig. 7.10), cuya excelente utilidad terapéutica en muy diversos tumores está bien demostrada (Minotti et al., 2004). Debe mencionarse sin embargo que, como ocurre con otras antriciclinas, su uso presenta toxicidad cardíaca crónica, acumulativa y dependiente de la dosis, por lo que se han diseñado diversos tipos de métodos para su transporte y liberación, particularmente liposomas (Niu et al., 2010; Pakunlu et al., 2006; Rifkin et al., 2006), micelas poliméricas (Cuong et al., 2011; Gao et al., 2005; Kim et al., 2008; You et al., 2008) y nanopartículas portadoras (Nawara et al., 2012; Du et al., 2011; Nowicka et al., 2009).

\subsubsection{Determinación espectrofotométrica}

El fármaco presenta un espectro UV-Vis que permite una determinación relativamente fácil y con suficiente precisión en solución usando un espectrofotómetro Dinko 8500 (Dinko Instruments, España)se encuentran los 


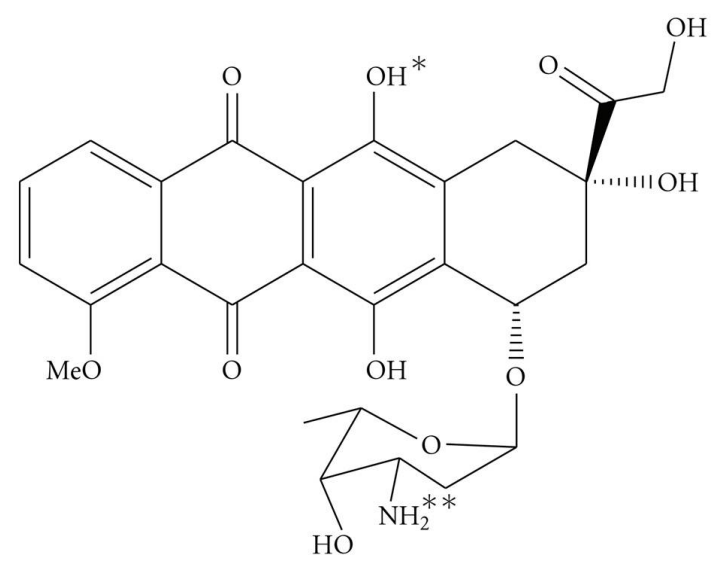

Figure 7.10: Estructura química de la doxorubicina.

espectros que se ilustran en la Fig. 7.11. Nótese la presencia de un máximo muy bien determinado en $489 \mathrm{~nm}$, así como el hecho de que la ley de Beer deja de cumplirse (el espectro se deforma y es imposible identificar el máximo mencionado) para concentraciones superiores a $3 \times 10^{-4} \mathrm{M}$. La figura muestra los datos y la recta de calibrado de absorbancia $(A)$ frente a concentración molar $(C):[A=(0.07 \pm 0.22)+(9400 \pm 220) \times C]$.

\subsubsection{Incorporación de agente antineoplásico}

Puede decirse que hay dos métodos de incorporación de fármacos a nanopartículas (Arias et al., 2010). Uno (absorción) supone la incorporación del fármaco en el interior de las partículas durante su síntesis. El otro es la adsorción superficial, es decir, adición del principio activo a partículas ya formadas. Dada la estructura de nuestras partículas, este es el único método aplicable y fue por ello el que se sigue en este trabajo. Se prepara en todos los casos una disolución de DOX de la concentración adecuada tal que mezclando $0.3 \mathrm{~mL}$ de ella con $0.2 \mathrm{~mL}$ de suspensión de NPs se llega a la concentración final buscada. Las partículas se mantienen en contacto con la disolcuión de fármaco durante 24 h, tras lo cual se centrifugan a 14000 rpm y se mide la absorbancia de los sobrenadantes.

La adsorción de Dox sobre los dos tipos de nanocompuestos se muestra en la Fig. 7.12, donde se aprecia que el segundo diseño es sin duda mejor portador, alcanzando una densidad de adsorción de saturación de $80 \mu \mathrm{mol} / \mathrm{g}$, frente a 40 en el caso del primer tipo de partículas. La mejor calidad del recubrimiento con oro es quizá la explicación de este hecho. 

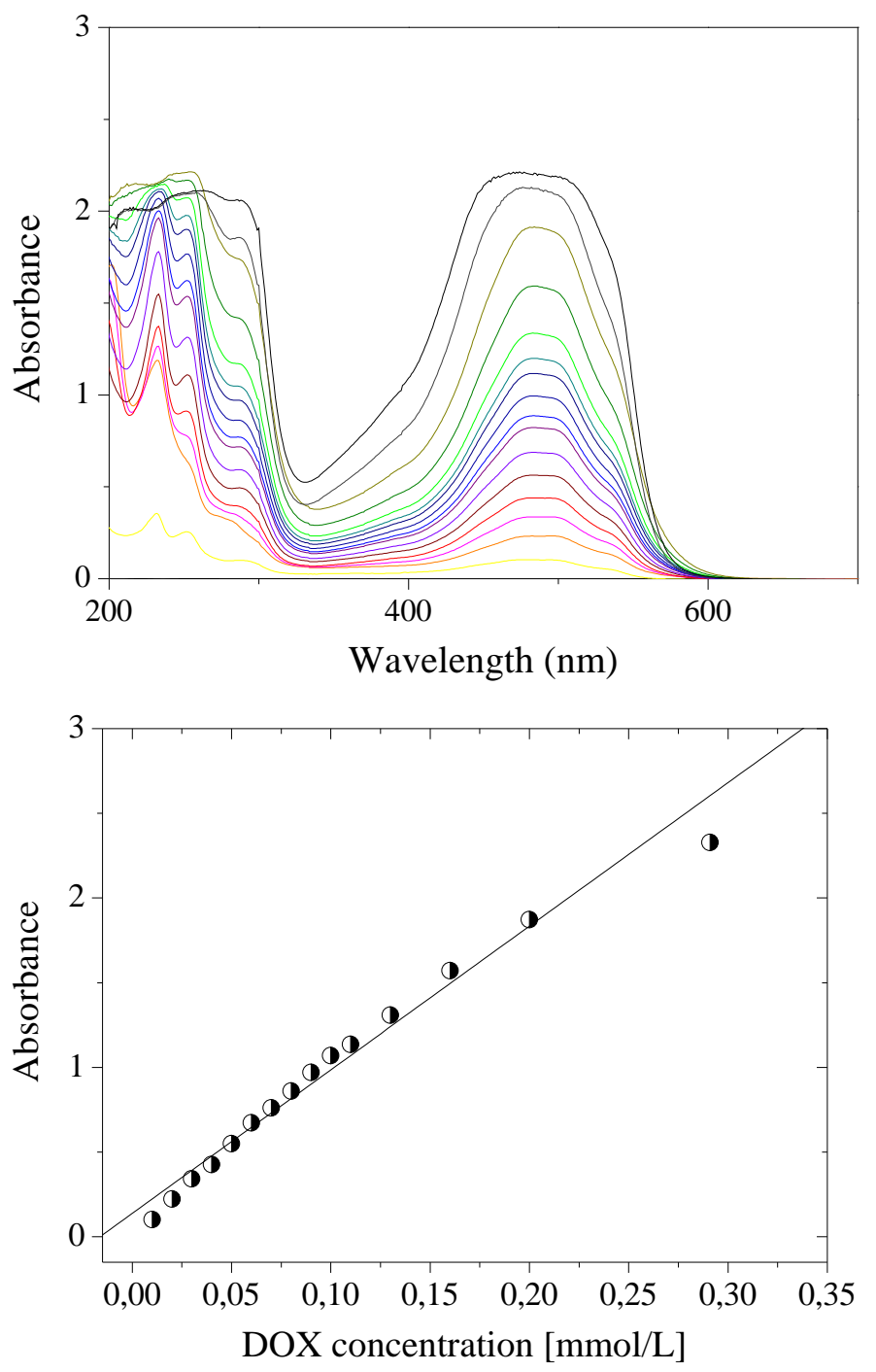

Figure 7.11: Superior: Absorbancia UV-Vis de disoluciones acuosas de DOX con concentraciones $(\mathrm{mM})$ (desde la curva inferior: 0.01, 0.02, 0.03, 0.04, 0.05, $0.06,0.07,0.08,0.09,0.1,0.11,0.13,0.16,0.2$. 0.3, 0.4. Inferior: Absorbancia en función de la concentración de DOX a una longitud de onda. 

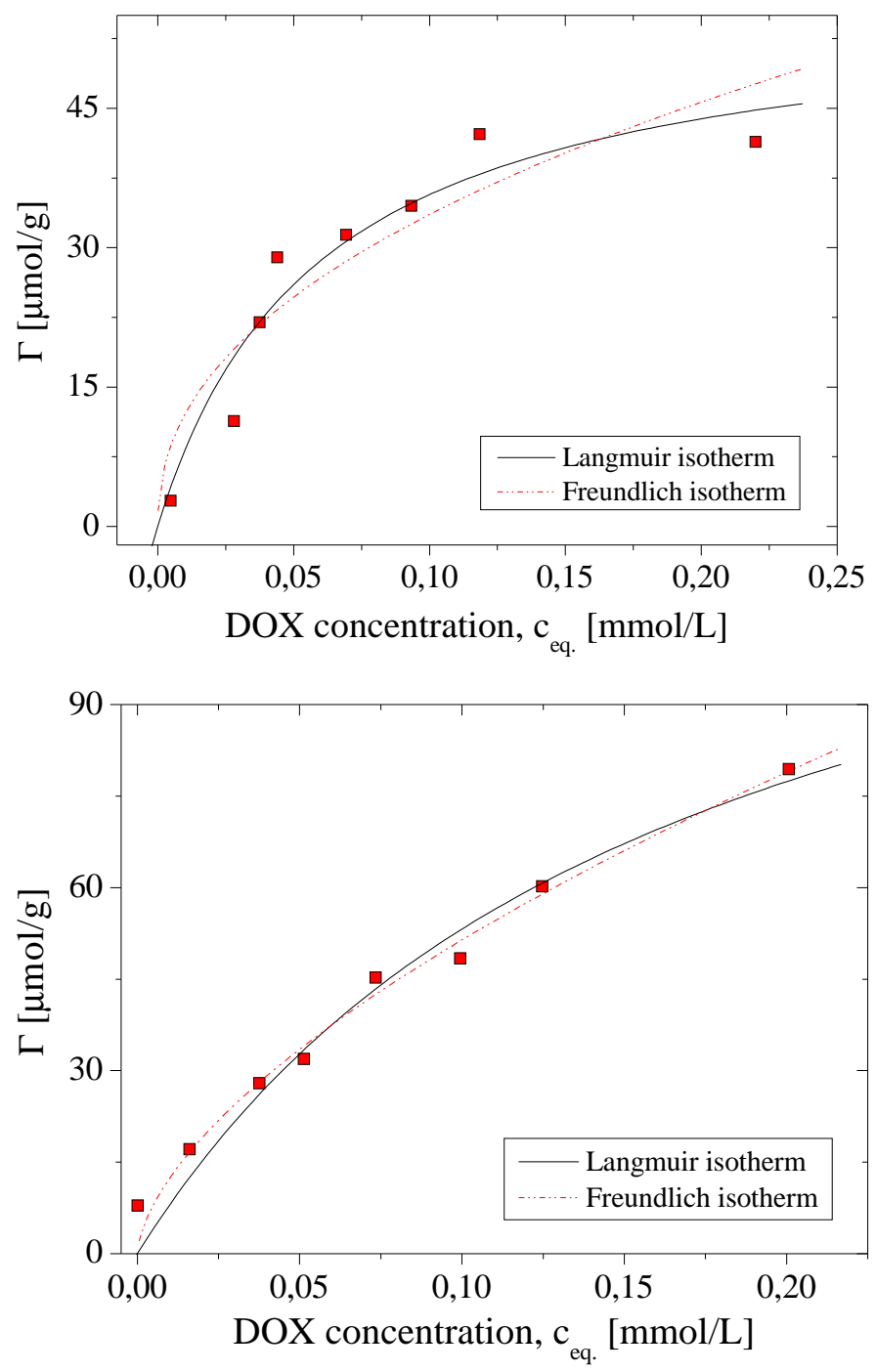

Figure 7.12: Adsorción de Dox sobre los dos diseños, junto con las curvas de ajuste a las isotermas de Langmuir y Freundlich. 

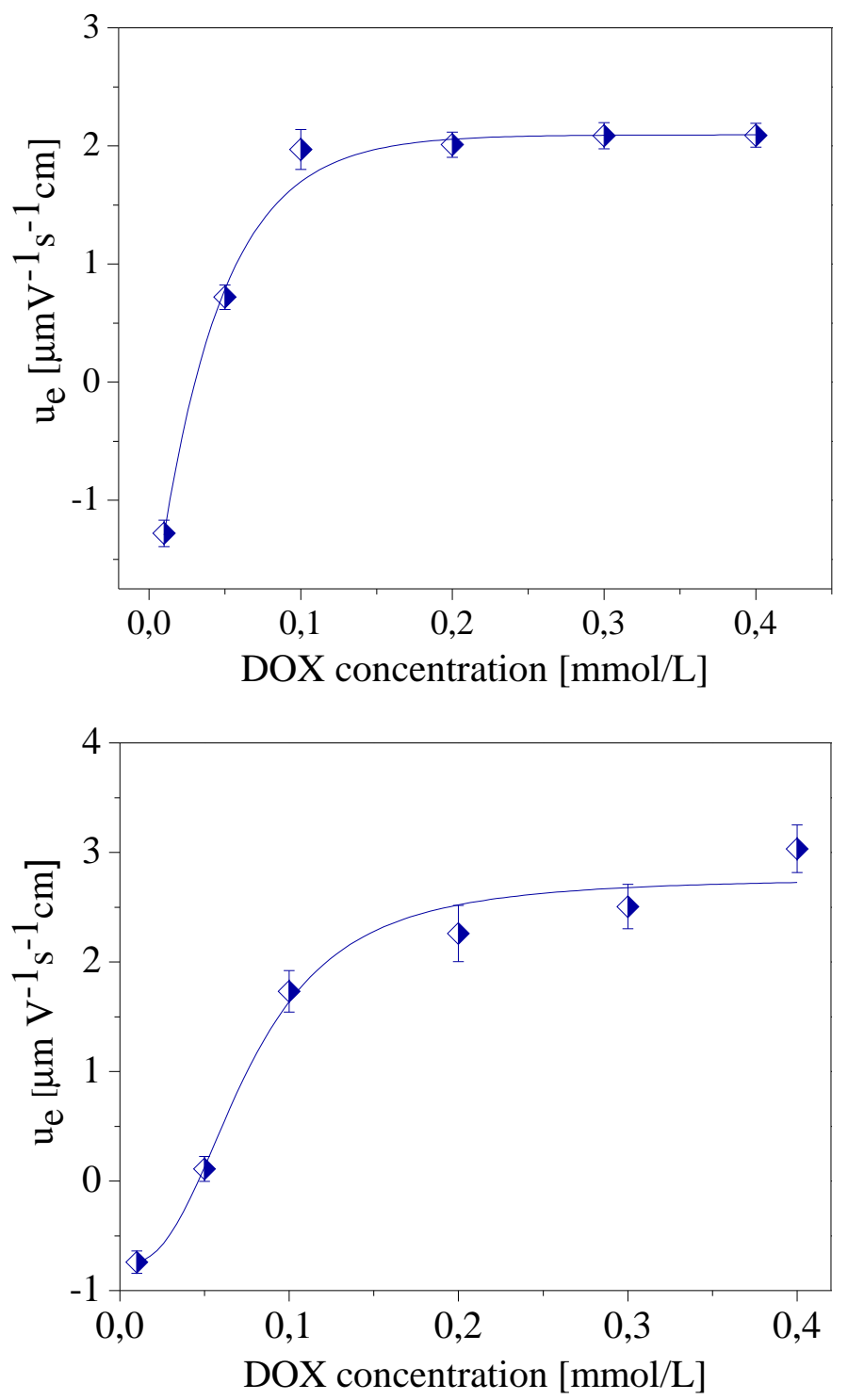

Figure 7.13: Movilidad electroforética de las nanoaestructuras tras 24 en contacto con disoluciones de DOX de las concentraciones que se indican (superior: tipo I; inferior: tipo II). 


\subsubsection{Análisis electrocinético}

Considerando el carácter catiónico de la doxorrubicina, es de esperar que el mecanismo principal de adsorción sea electrostático, y que la adsorción se manifieste en la carga superficial y por tanto, en la movilidad electroforética. La Fig. 7.13 confirma esta hipótesis, mostrando que la movilidad es negativa en cualquiera de los diseños sólo si la concentración de DOX es baja, tendiendo claramente a valores positivos en valores mayores, alcanzando un plateau para concentraciones del orden de $0.2 \mathrm{mmol} / \mathrm{L}$ (diseño I) y tendencia creciente en la zona positiva en el diseño II. Mecanismos similares de adsorción han sido recientemente propuestos por (Tapeinos et al., 2013) usando microesferas de polímero como vehículos.

\subsection{Liberación in vitro}

\subsubsection{Metodología}

El procedimiento seguido para evaluar la liberación del fármaco adsorbido consiste en añadir $0.6 \mathrm{~mL}$ de suspensión de NPs $(0.1 \%$ en peso de sólidos en el primer diseño y $0.065 \%$ en el segundo) a $0.4 \mathrm{~mL}$ de disolución DOX $0.75 \mathrm{mM}$. La suspensión resultante se mantiene en agitación $24 \mathrm{~h}$. Las partículas se centrifugan y se redispersan en $1 \mathrm{~mL}$ de buffer PBS a $\mathrm{pH}$ 7.4 y $37^{\circ} \mathrm{C}$. A intervalos de tiempo especificados, se toman $150 \mu \mathrm{L}$ de suspensión, se centrifuga y se mide la absorbancia del sobrenadante a 489 nm. Las partículas se reconstituyen y se devuelven a la suspensión madre.

\subsubsection{Resultados}

La Fig. 7.14 muestra el ritmo de liberación de DOX para los dos tipos de nanopartículas diseñadas. En la Tesis se detallan los distintos modelos de liberación que serían aplicables. En nuestro caso, siguiendo criterios de coeficiente de determinación ajustado y Akaike, los mejores ajustes fueron las cinéticas de orden uno y de Weibull (Fig. 7.15), es decir, el fármaco se libera a un ritmo proporcional a la cantidad de fármaco que queda sobre las partículas. También se encuentra un buen ajuste al modelo de KorsmeyersPeppas (ley de potencias) con un exponente (que se obtiene de los datos de tiempos cortos, como se indica en la Fig. 7.16) $n$ de valor 0.59 y 0.73 para los modelos I y II, respectivamente.

Modelo de primer orden En este modelo, la liberación ocurre de acuerdo con la ecuación: 

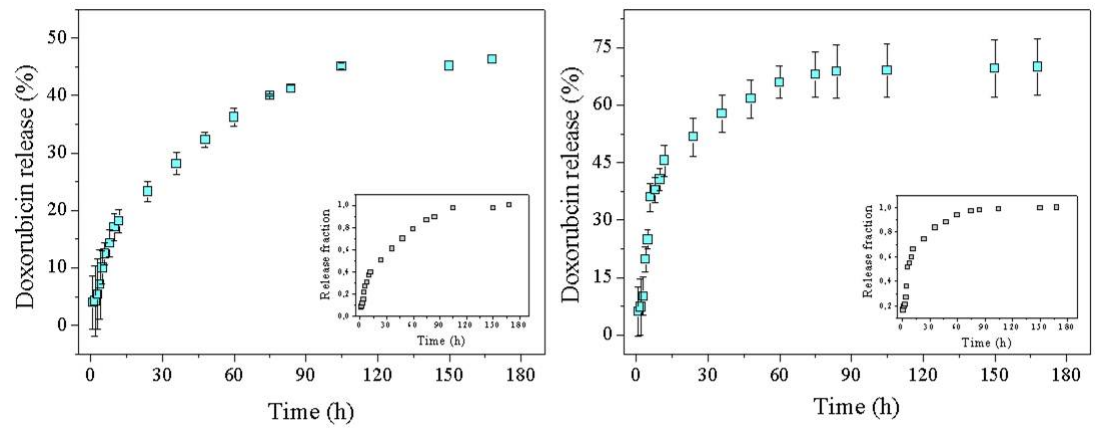

Figure 7.14: Perfiles de liberación de DOX desde nanocompuestos magnéticos. Izquierda: diseño I; derecha: diseño II.

$$
\frac{C}{t}=-K C,
$$

donde $K$ representa la constante cinética y $\mathrm{C}$ es la concentración de agente citotóxico.

El modelo de Korsmeyer-Peppas tiene su origen en una ecuación debida a (Korsmeyer et al., 1983). Puede expresarse como:

$$
M_{t} / M_{\infty}=k t^{n}
$$

donde $M_{t} / M_{\infty}$ representa la fracción de fármaco liberado, $k$ es la constante de velocidad, y el exponente $n$ da información acerca del tipo de mecanismo de liberación.

Finalmente, la ecuación empírica derivada por Weibull en 1951 se aplicó por primera vez a la liberación/disolución en vehículos de fármacos por (Lagenbucher, 1972). Posteriormente, se ha usado con éxito a la simulación de muchos ejemplos de liberación (Goldsmith et al., 1978; Vudathala \& Rogers, 1992). La ecuación del modelo de Weibull es:

$$
M=\left[1-e^{\frac{(t-T)^{b}}{a}}\right]
$$

donde $M$ es la fracción de agente citotóxico acumulada en la solución en función del tiempo $t$. $T$ representa el periodo de retraso o latencia en la liberación, y $a$ y $b$ son parámetros que ajustan la forma de la curva cinética. 

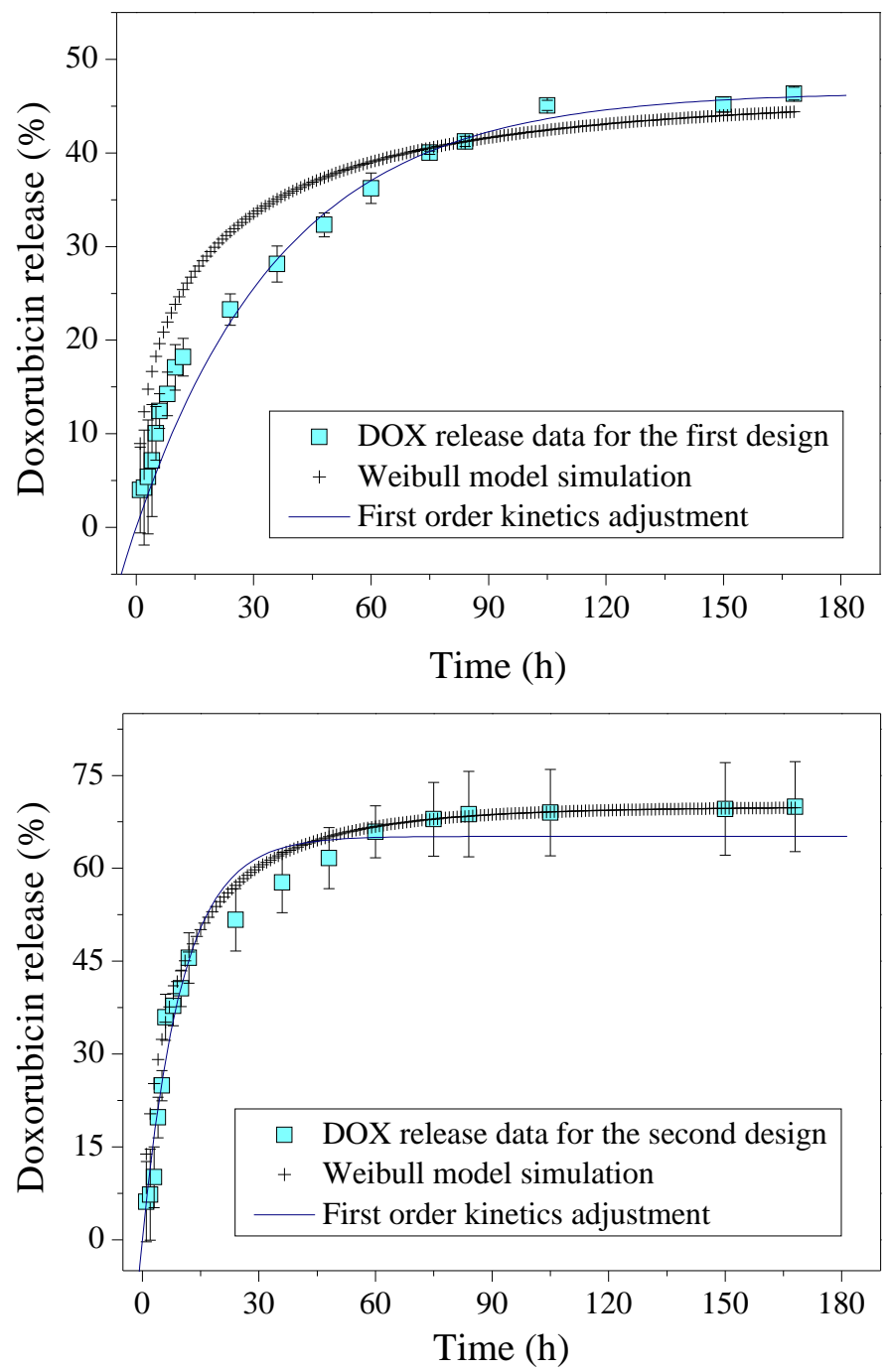

Figure 7.15: Ajustes de los datos de liberación a los modelos de Weibull y primer orden. 

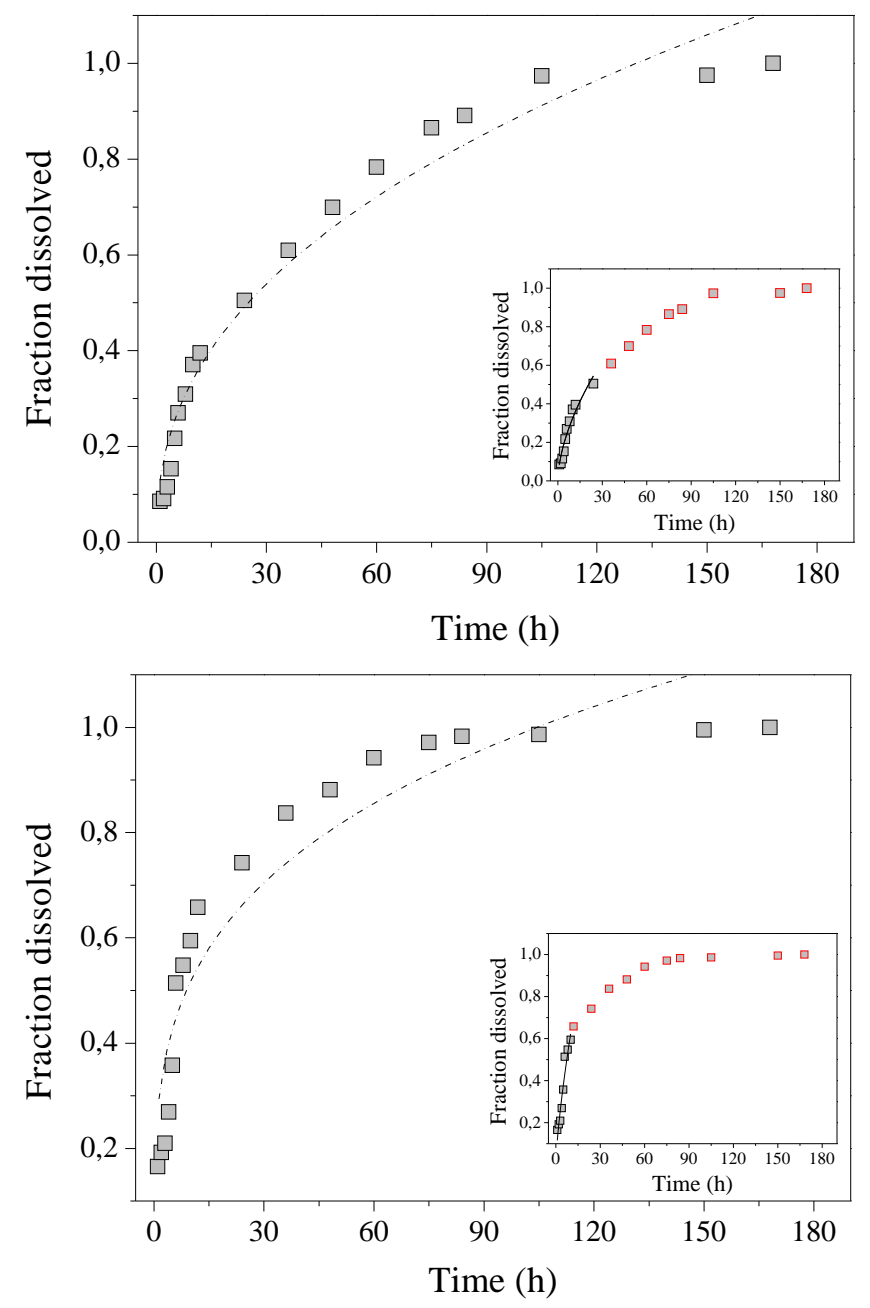

Figure 7.16: Fracción de DOX liberada de ambos diseños (I: gráfica superior; II: inferior) y ajuste al modelo Korsmeyer-Peppas (ley potencial). En las gráficas insertadas se representa el detalle de la zona de tiempos cortos para la obtención de $n$. 


\subsection{Cultivos celulares}

\subsubsection{Metodología}

En esta ultima parte de la tesis, se investiga el verdadero comportamiento de nuestros vehículos magnéticos de DOX en contacto con células tumorales. Se usan dos lineas celulares bien caracterizadas, a saber, PLC-PRF-5 (hígado) y DLD-1 (colorrectal). Los medios de cultivo son, respectivamente, RPMI1640 Y DMEM (Invitrogen, USA) suplementados con suero fetal bovino, penicilina y estreptomicina. La temperatura de incubación fue $37{ }^{\circ} \mathrm{C}$. El tiempo de contacto del fármaco libre (usado para comparación) y las nanopartículas fue de $2 \mathrm{~h}$, transcurridas las cuales, las células se fijan con paraformaldehído y se tiñen con DAPI (Sigma, USA). La autofluorescencia de las partículas se detecta con un microscopio láser confocal Leica Spectral (Alemania). El fármaco es fluorescente a $533 \mathrm{~nm}$ si se excita con láser de $488 \mathrm{~nm}$. Se llevó a cabo igualmente un estudio de viabilidad de las mismas células, utilizando el método de la sulfo-rodamina B (Valenzuela et al., 1995). Este estudio sólo se realizó con el segundo diseño.

\subsubsection{Resultados}

Las Figs. 7.17, 7.19 y 7.18 demuestran que la doxorrubicina se libera en los núcleos de las células cancerosas, que presentan la fluorescencia roja igualmente tras contacto con el fármaco libre como con las nanopartículas, y en los dos tipos de células.

Finalmente, la acción antitumoral de los vehículos sintetizados se confirma igualmente mediante el estudio de la viabilidad de las células tumorales en contacto con las partículas. Se uso para ello el método de la sulfo-rodamina B (Valenzuela et al., 1995) en el caso de los portadores de tipo II, dada su mayor estabilidad y los mejores resultados descritos en relación con su capacidad de adsorber fármaco. Las Figs. 7.21 y 7.20 muestran los ensayos de viabilidad de las dos lineas celulares para tres diluciones de la suspensión de nanopartículas, en comparación con el tratamiento realizado con DOX libre a concentraciones comparables. Nótese que las partículas sin fármaco solo afectan a la viabilidad al cabo de $48 \mathrm{~h}$ de contacto, mientras que su efecto es claro cuando llevan fármaco; el efecto es mucho más notable en el caso de las células DLD-1 (Fig. 7.21): mientras que para células de hígado la viabilidad es del $31 \%$ con DOX y $20 \%$ con nanopartículas, las cifras cambian a $51 \%$ y $22 \%$ cuando se trata de células de colon. Estos datos se refieren a las condiciones óptimas, esto es, $48 \mathrm{~h}$ de 

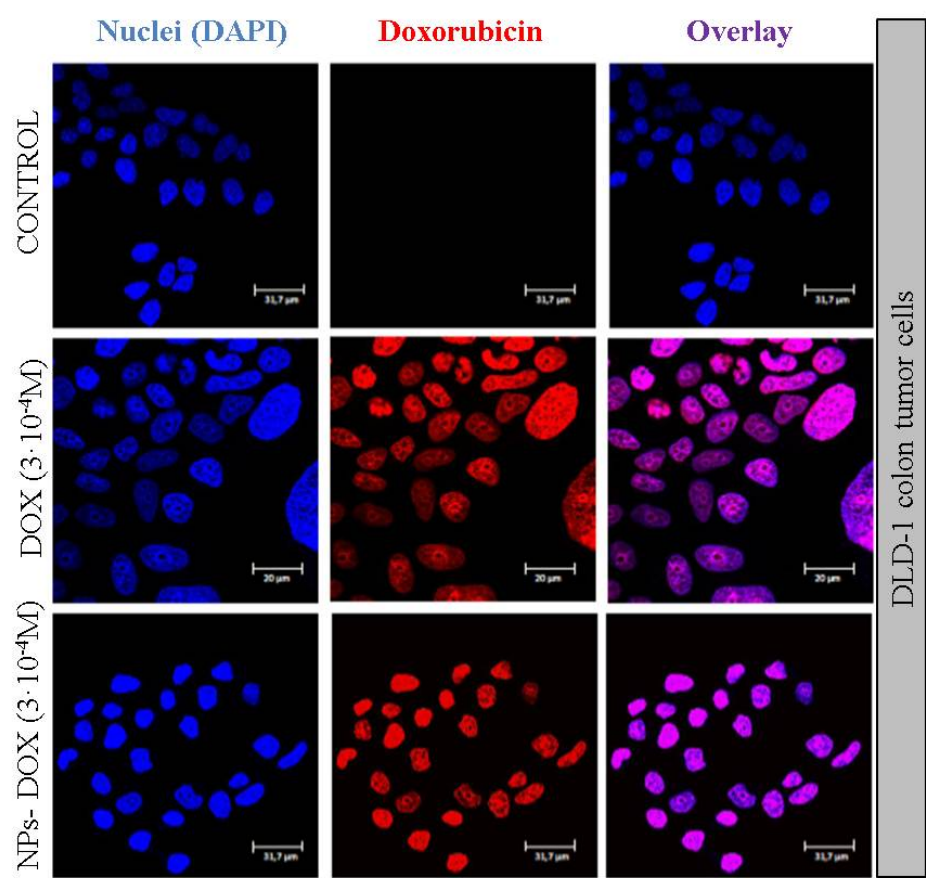

Figure 7.17: Imágenes de microscopia confocal de células tumorales DLD-1 (colon) no tratadas, y tratadas con nanopartículas portadoras del fármaco a dos concentraciones de DOX. El color azul corresponde al tintado de los núcleos. La concentración de partículas fue de $1.8 \mathrm{mg} / \mathrm{mL}$. 


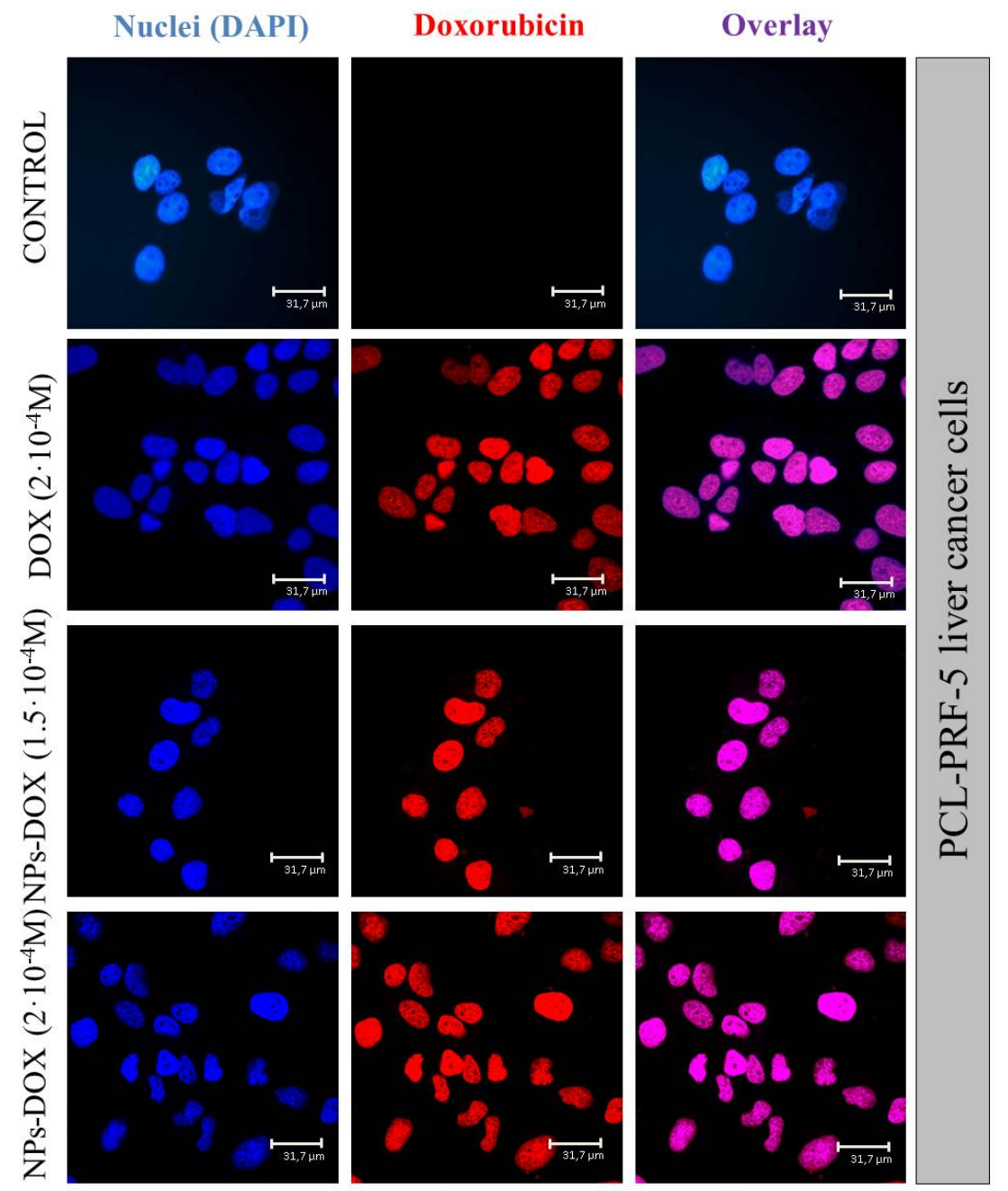

Figure 7.18: Igual que Fig. 7.17, para células de tumor hepático (PLC-PRF5). En la segunda fila se muestran resultados para células tratadas con fármaco libre. Concentración de partículas: $1.25 \mathrm{mg} / \mathrm{mL}$. diseño II 


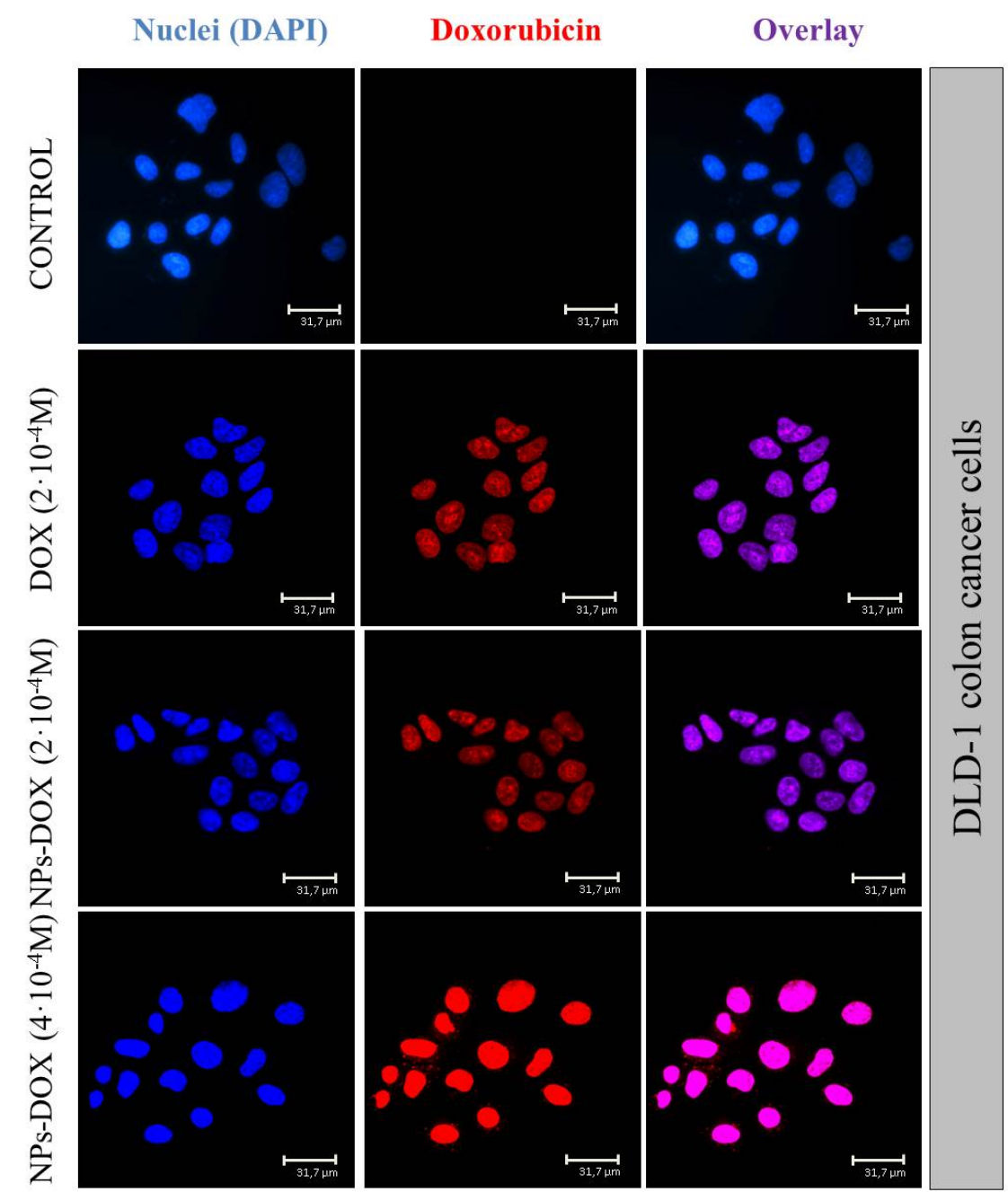

Figure 7.19: Como la Fig. 7.18, pero para tumor de colon (DLD-1). 

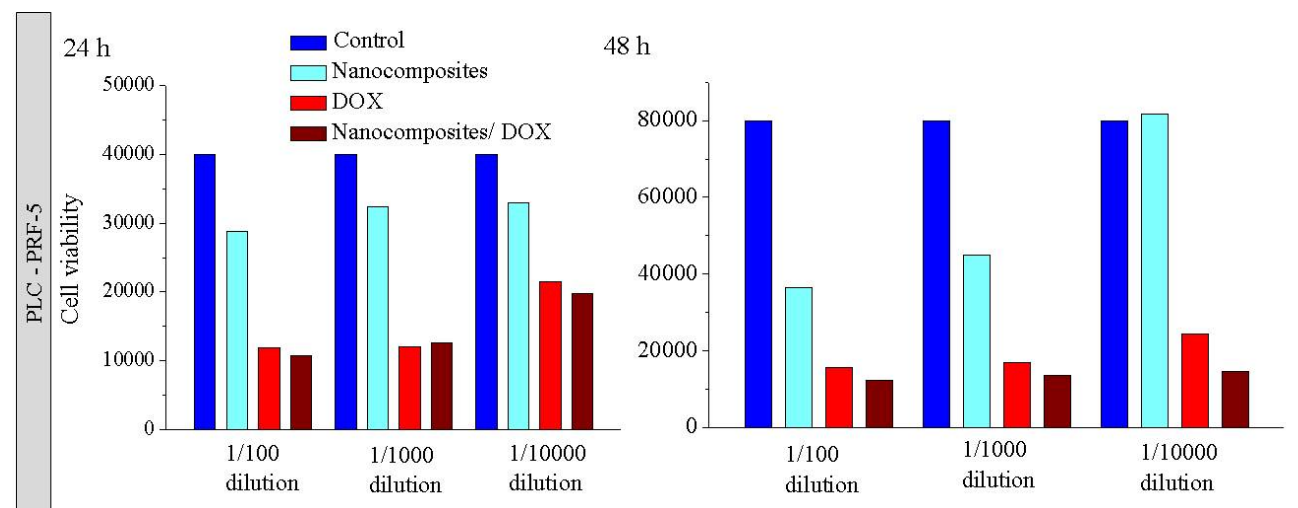

Figure 7.20: Ensayos de viabilidad para células de tumor hepático tratadas durante 24 y $48 \mathrm{~h}$ con partículas solas, DOX libre y DOX adsorbida sobre nanopartículas. La concentración de partículas en la suspensión no diluida es $1.25 \mathrm{mg} / \mathrm{mL}$.

contacto y dilución $1 / 10000$.

Finalmente, las fotografías de campo claro como las de la Fig. 7.22 muestran que la viabilidad celular se reduce significativamente tras el contacto con las NPs cargadas con DOX, en comparación con los resultados obtenidos con fármaco libre en disolución. El efecto de las partículas sin fármaco es pequeño comparativamente y probablemente se puede asociar con el estrés celular inducido por la sobre-estimulación endocítica. 

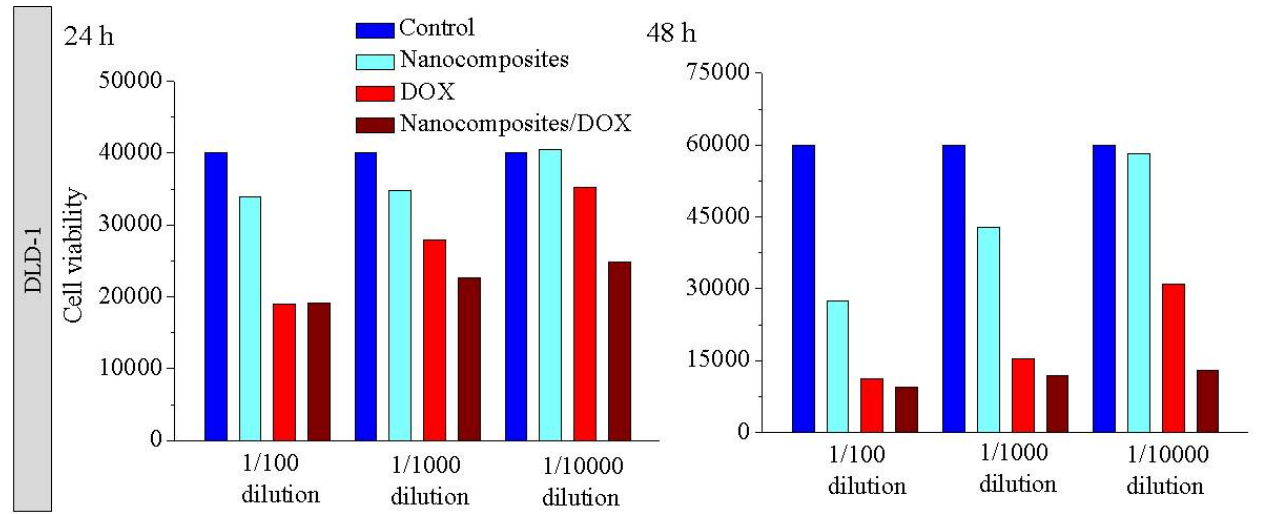

Figure 7.21: Como Fig. 7.20, pero para células de cáncer colorrectal, DLD-1.

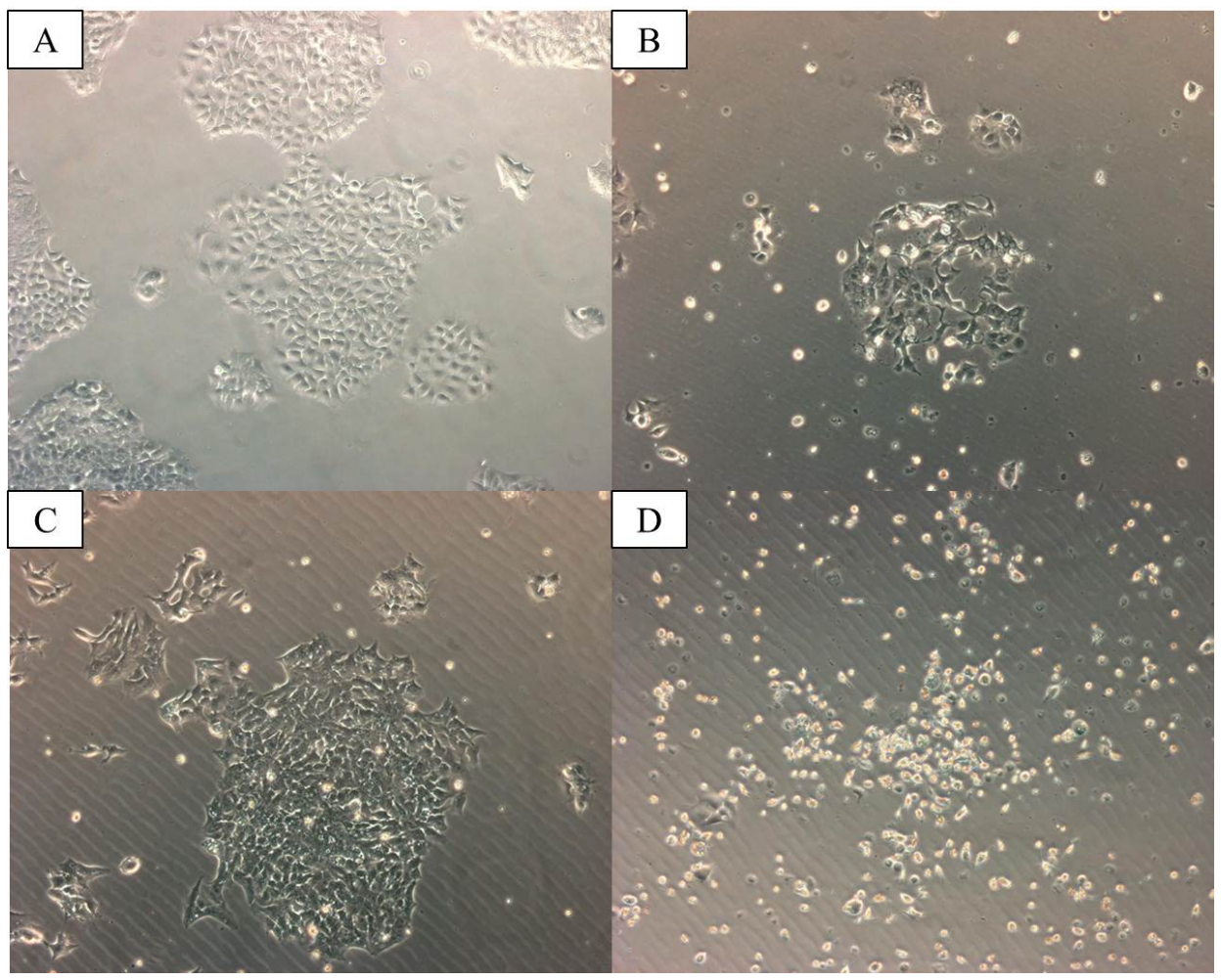

Figure 7.22: Imágenes de microscopía de campo claro de células DLD 1. A: control; B: en contacto con $0.3 \mathrm{mM}$ DOX en disolución; C: en contacto con nanopartículas; D: con DOX 0.3 mM adsorbida sobre nanopartículas. Diseño II. Fotos tras 48 h en contacto. 
Chapter 8

Conclusions 

We will present the essential contributions obtained from this Thesis, which may be abridged in the following conclusions:

Presentamos a continuación las contribuciones principales del presente trabajo, que resumimos en las siguientes conclusiones:

About the synthesis of the nanocompounds :

En relación a la síntesis de los nanocompuestos:

a) We have synthesized maghemite nanoparticles and through its coating process with silica and gold nanoparticles two different delivery systems for doxorubicin have been obtained.

Se han sintetizado nanopartículas de maghemita y, a través de su recubrimiento con silice y nanopartículas de oro, se han obtenido dos sistemas de liberación para la doxorrubicina.

b) In this manner, we have achieved the design of a controlled drug delivery system which is composed of a magnetic core and a biocompatible silica/gold coating.

De esta forma, se ha logrado un diseño controlado del sistema de liberación del fármaco compuesto por un núcleo magnético y un recubrimiento biocompatible de sílice/oro.

\section{About the structure and chemical composition :}

\section{En relación a la estructura y la composición química :}

c) Through X-ray Powder Diffraction we have confirmed that the majority of our magnetic core corresponds to maghemite, according to the six XRD peaks which are in agreement with the reference data in more than $58 \%$. This conclusion is also confirmed by the position of the principal peak of the synthesized maghemite.

Mediante la difracción de rayos $X$ por polvo, se ha confirmado que la mayoría de los núcleos magnéticos son de maghemita, de acuerdo con los seis picos de la difracción de rayos $X$, los cuales coinciden con los datos disponibles en la bibliografía en más de un $58 \%$. Esta conclusión se confirma también por la posición del pico principal en la sintesis de la maghemita. 
d) X-ray Photoelectron Spectroscopy permits to determine that the synthesized sample is only composed of $\mathrm{Fe}^{3+}$ states, so that the obtained iron oxide is principally maghemite.

La Espectroscopía de fotoelectrones emitidos por rayos $X$ permite determinar que la muestra sintetizada está compuesta únicamente por estados de $\mathrm{Fe}^{3+}$ de forma que el óxido de hierro obtenido es principalmente maghemita.

\section{Conclusions about the electrokinetic properties of the synthesized particles :}

\section{Conclusiones sobre las propiedades electrocinéticas de las partículas sintetizadas :}

e) With the help of electrophoretic study we could control the stability of the two fundamental steps in the overall nanocarrier formation in our work, namely, the maghemite synthesis and its silica/gold coating.

Con la ayuda de la electroforesis, se pudo controlar la estabilidad de los dos pasos fundamentales en la formación global de nanotransportadores, concretamente, en nuestro trabajo, en la sintesis de maghemita y el recubrimiento con sílice/oro.

f) Depositions of three oppositively charged polyelectrolyte layers, in the procedure I, and the cationic polyelectrolyte APTMS, in case of the procedure II, have been employed to prepare the magnetic silica spheres and functionalize them as a seat for gold nanoparticles.

Los depósitos de tres capas de polielectrolito opuestamente cargadas, en el procedimiento I, y de poliectrolito catiónico APTMS, para el procedimiento II, han sido usadas para la preparación de esferas de sílice magnetizadas y funcionalizadas como asiento para las nanopartículas de oro.

g) The electrophoretic mobility evolution confirms the formation of a gold layer onto the nanocomposites surface for both procedures followed.

La evolución en la movilidad electroforética confirma la formación de capas de oro sobre la superficie de los nanocompuestos en ambos de los procedimientos seguidos.

h) The results of mobility as a function of ionic strength agree with existing models regarding the finite value of the electrophoretic mobility 
at very high ionic strengths for soft particles (with polyelectrolyte coating).

Los resultados de la movilidad en función de la fuerza iónica coinciden con los modelos existentes en cuanto al valor finito de la movilidad electroforética para fuerzas iónicas muy altas en el caso de partículas blandas (con recubrimiento de polielectrolito).

\section{Magnetic properties of the particles :}

\section{Propiedades magnéticas de las partículas :}

i) Magnetic studies point out that for all materials (either unmodified or modified maghemite nanoparticles) we can observe superparamagnetic behaviour at room temperature, suggesting that the thermal fluctuations turn out to be prevailing over spontaneous magnetization at finite fields, while the net magnetization when the external magnetic field is not applied is zero.

Los estudios magnéticos han señalado que en todos los materiales (nanopartículas de maghemita modificadas o sin modificar) puede observarse un comportamiento superparamagnético a temperatura ambiente, sugiriendo que las fluctuaciones térmicas resultan prevalecer sobre la magnetización espontánea en campos finitos, mientras que la magnetización neta es cero cuando no hay campos eléctricos aplicados.

j) The saturation magnetization of the final nanocomposites is more than ten times lower than the saturation magnetization value for the bare maghemite nanoparticles.

La saturación magnética de los nanocompuestos finales es más de diez veces menor que la saturación magnética para las nanopartículas de maghemita desnudas.

k) The values of relaxivity of both bare and silica-coated maghemite have been compared to those of the commercial products utilized as MRI contrast agents.

Los valores de la relajación de ambos casos, maghemita desnuda y recubierta con sílice, han sido comparados con productos comerciales utilizados como agentes de contraste en Imagen por Resonancia Magnética.

About the drug transport capacity and the release mechanism of doxorubicin : 
En relación a la capacidad para transportar el fármaco y el mecanismo de liberación de la doxorrubicina :

l) Spectrophotometric characterization of the determination of the capacity for vehiculization of the antitumor drug by the designed nanoparticulate system has been performed.

Se ha llevado a cabo la caracterización espectrofotométrica para la determinación de la capacidad de vehiculización del fármaco antitumoral para el sistema de nanopartículas diseñadas.

m) Nanovehicle stability for both designs has been determined via optical absorbance study.

La estabilidad de los nanovehículos para ambos diseños ha sido determinada mediante un estudio de absorbancia óptica.

n) The evaluation of the surface drug adsorption for the two designed doxorubicin delivery systems has been carried out both qualitatively (electrophoretic mobility) and quantitatively (UV-Vis spectrophotometry). The best fit for the adsorption isotherm has been found to correspond to a Langmuir-type dependence.

La evaluación de la adsorción del fármaco para los dos diseños de liberación de doxorrubicina han sido realizados tanto cualitativamente (movilidad electroforética) como cuantitativamente (mediante espectrofotometría de UV-VIS). El mejor ajuste para las isotermas de adsorción corresponde a una dependencia del tipo Langmuir.

o) In vitro release study has been performed and the best model for the release mechanism has been chosen. Confocal fluorescence microscopy demonstrated that both DOX vehicle formulations are capable of penetrating the cell membranes and releasing the DOX payload into the nuclei of liver and colorectal tumor cells. Clear field microscopy have been employed in order to present cell viability results in more illustrative way.

Se ha realizado un estudio de liberación in vitro y se ha elegido el mejor modelo para el mecanismo de liberación. La microscopía confocal de fluorescencia ha demostrado que ambas vehiculizaciones de doxorrubicina son capaces de penetrar la membrana celular y liberar la carga de doxorrubicina en el núcleo de las células tumorales del hígado y del colon. La miscroscopía de campo claro se ha empleado para presentar los resultados de viabilidad de las células tumorales de una forma más ilustrativa. 


\section{References}

Alexis, F., Pridgen, E., Molnar, L. \& Farokhzad, O. (2008). Factors affecting the clearance and biodistribution of polymeric nanoparticles. Mol Pharm, 5(4), 505-515.

Arcamone, F., Animati, F., Berettoni, M., Bigioni, M., Capranico, G., Casazza, A., Caserini, C., Cipollone, A., De Cesare, M. \& FranCіOтTI, M.E.A. (1997). Doxorubicin disaccharide analogue: apoptosis-related imprimprove of efficacy in vivo. J. Natl. Cancer Inst, 89, 1217-1223.

ARIAS, J. (2008). Novel strategies to improve the anticancer action of 5-fluorouacil by using drug delivery systems. Molecules, 13, 2340-2369.

ARIAS, J. (2011). Drug targetting strategies in cancer treatment: An overview. Mini Rev. Med. Chem., 11, 1-17.

Arias, J., Lopez-Viota, M., Saez-Fernandez, E. \& Ruiz, M. (2010). Formulation and physicochemical characterization of poly( $\varepsilon$-caprolactone) nanoparticles loaded with ftorafur and diclofenac sodium. Colloids Surf B: Biointerfaces, 75, 204-208. 
Arias, J., Clares, B., Morales, M., Gallardo, V. \& Ruiz, M. (2011a). Lipid-based drug delivery systems for cancer treatment. Curr. Drug Targets, 12, 1151-1165.

Arias, J., López-Viota, M., Sáez-Fernández, E., Ruiz, M. \& Delgado, A. (2011b). Engineering of an antitumor (core/shell) magnetic nanoformulation based on the chemotherapy agent ftorafur. Colloids Surf., A, 384, 157-163.

Arruebo, M., Galán, M., Navascués, N., Téllez, C., Marquina, C., Ibarra, M. \& Santamaría, J. (2006). Development of magnetic nanostructured silica-based materials as potential vectors for drug-delivery applications. Chem. Mater., 18, 1911-1919.

Arruebo, M., Fernandez-Pacheco, R., Ibarra, M. \& Santamaria, J. (2007). Magnetic nanoparticles for drug delivery. NanoToday, 2, 22-32.

Atkinson, W., Brezovich, I. \& Chakraboorty, D. (1984). Usable frequencies in hyperthermia with thermal seeds. IEEE Trans. Biomed. Eng. BME, 31, $70-5$.

Baker, R. \& Longsdale, H. (1974). Contolled release: mechanisms and rates, in: Controlled Release of Biologically Active Agents. Plenum Press, New York,.

Banerjee, R., Katsenovich, Y., Lagos, L., Mclintosh, M., Zhang, X. \& LI, C. (2010). Nanomedicine: Magnetic nanoparticles and their biomedical applications. Current Medicinal Chemistry, 17(27), 3120-3141.

Bantle, S., Schmidt, M. \& Burchard, W. (1982). Simultaneous static and dynamic light scattering. Macromolecules, 15, 1604-1609. 
Barbé, C., Bartlett, J., Kong, L., Finnie, K., Qiang Lin, H., larkin, M., Calleja, S., Bush, A. \& Calleja, G. (2004). Silica particles: A novel drug-delivery system. Adv. Mater., 16, 1959-1966.

Barreto, J., O’Malley, W., Kubeil, M., Graham, H., B. Stephan \& SpICCIA, L. (2011). Nanomaterials: applications in cancer imaging and therapy. Adv. Matter., 23, H18-H40.

Berkowitz, A., Schuele, W. \& Flanders, P. (1968). Influence of crystalline size on the magnetic properties of acicular gamma-fe2o3 particles. J. Appl. Phys., 39, 1261-1263.

Berry, C. \& Curtis, A. (2003). Functionalization of magnetic nanoparticles for applications in biomedicine. J Phys. D Appl. Phys., 36, R198-R206.

Berry, C., Wells, S., Charles, S. \& Curtis, A. (2003). Dextran and albumin derivatised iron oxide nanoparticles: influence on fibroblasts in vitro. Biomaterials, 24, 4551-4557.

Berry, C., Wells, S., Charles, S., Aitchison, G. \& Curtis, A. (2004). Cell response to dextran-derivatised iron oxide nanoparticles post internalisation. Biomaterials, 25(23), 5405-5413.

Bhattacharya, R., Patra, C., Earl, A., Wang, S., Katarya, A., Lu, L., Kizhakkedathu, J., Yaszemski, M., Greipp, P., Mukhopadhyay, D. \& Mukherjee, P. (2007). Attaching folic acid on gold nanoparticles using covalent interaction via different polyethylene glycol backbones and targeting of cancer cells. Nanomed. Nanotechnol. Biol. Med., 3, 224-238.

BI, S., WeI, X., LI, N. \& LEI, Z. (2008). In situ formation of $f e_{3} o_{4}$ naoparticles within thermosensitive hairy hybrid particles. Mater. Lett., 62, 2963-2966. 
Blunk, T., Hochstrasser, D., Sanchez, J., Muller, B. \& Muller, R. (1993). Colloidal carriers for intravenous drug delivering: Plasma protein adsorption patterns on surface-modified latex particles evaluated by two-dimensional polyacrylamide gel electrophoresis. Electrophoresis, 14, 1382-87.

Bochard, J., G. Kreuter (1993). Interaction of serum components with poly(methylmethacrylate) nanoparticles and the resulting body distribution after intravenous injection in rats. J. Drug Target., 1, 15-19.

Boissière, M., Allouche, J., Brayner, R., Chanéac, C., Livage, J. \& Coradin, T. (2007). Design of iron oxide/silica/alginate hybrid magnetic carriers (hymac). J. Nanosci. Nanotechnol., 7, 4649-54.

Brigger, I., Dubernet, C. \& Couvreur, P. (2002). Nanoparticles in cancer therapy and diagnosis. Adv Drug Deliv Rev., 54, 631-651.

Brown, W. (1993). Dynamic light scattering: The method and some applications. Clarendon Press, Oxford, England.

Brown, W.J. (1963). Thermal fluctuations of a single-domain particle. Phys. Rev., 130, 1677-86.

Browne, E. \& SemelKa, R. (1999). MRI:Basic principles and applications. Wiley: New York.

Brulé, S., Levy, M., Wilhelm, C., Letourneur, D., Gazea, F., Ménager, C. \& Le Visage, C. (2011). Doxorubicin release triggered by alginate embedded magnetic nanoheaters: A combined therapy. Adv. Mater., 23, 787-790.

Busbee, B., Obare, S. \& Murphy, C. (2003). An improved synthesis of highaspect-ratio gold nanorods. Adv. Mater., 15, 414-416. 
Callister, W.J. (2005). Materials Science and Engineering, 7th Ed., Chap. 20 : Magnetic properties. John Wiley \& Sons.

Caruso, F., Lichtenfeld, H., Donath, E. \& Mohwald, H. (1999). Investigation of electrostatic interactions in polyelectrolyte multilayer films: Binding of anionic fluorescent probes to layers assembled onto colloids. Macromolecules, 32, 2317-2328.

Chan, D., Kirpotin, D. \& Bunn, P. (1993). Synthesis and evaluation of colloidal magnetic iron oxides for the site-specific radiofrequency-induced hyperthermia of cancer. J. Magn. Magn. Mater., 122, 374-378.

Chang, J., Chang, K. \& Hwang, D. (2007). In vitro cytotoxity of silica nanoparticles at high concentrations srongly ddepend on the metabolic activity type of the cell line. Environ Sci Technol, 41, 2064-8.

Chari, R. (1998). Targeted delivery of chemotherapeutics: tumor-activated prodrug therapy. Adv. Drug Deliv. Rev., 31, 89-104.

Chastellain, M., Petri, A., Gupta, A., Rao, K. \& Hofmann, H. (2004). Superparamagnetic silica-iron oxide nanocomposites for application in hyperthermia. Adv. Eng. Mater., 6, 235-241.

Chen, S., Zhu, J. \& Cheng, J. (2007). Preparation and in vitro evaluation of a novel combined multiparticulate delayed-onset sustained-release formulation of diltiazem hydrochloride. Die Pharmazie-An International Journal of Pharmaceutical Sciences, 62 (12), 907-913.

Cheon, J. \& Lee, J. (2008). Synergistically integrated nanoparticles as multimodal probes for nanobiotechnology. Acc Chem Res., 41, 1630-40. 
Chertok, B., David, A. \& Yang, V. (2010). Polyethyleneimine-modified iron oxide nanoparticles for brain tumor delivery using magnetic targeting and intracarotid administration. Biomaterials, 31, 6317-24.

Chibowski, E. (2003). Surface free energy of a solid from contact angle hysteresis. Adv. Colloid interface Sci., 103, 149-172.

Chibowski, E. (2007). On some relations between advancing, receding and young's contact angles. Adv. Colloid Interface Sci., 133, 51-59.

Chibowski, E. \& Gonzalez-Caballero, F. (1993). Theory and practice of thin-layer wicking. Langmuir, 9, 330-340.

Chibowski, E., Delgado, A., Rudzka, K., Szczes, A. \& Holysz, L. (2011). Surface modification of glass plates and silica particles by phospholipid adsorption. Journal of Colloid and Interface Science, 353, 281-289.

Chouly, C., Pouliquen, D., Lucet, I., Jeune, J. \& Jallet, P. (1996). Development of superparamagnetic nanoparticles for mri: Effect of particle size, charge and surface nature on distribution. J Microencapsulation, 13, 245-55.

Colombo, M., Carregal-Romero, S., Casula, M., Gutierrez, L., Morales, M., Bohm, I., Haverhagen, J.T., Prosperi, D. \& Parak, W. (2012). Biological applications of magnetic nanoparticles. Chem Soc Rev, 41, 4306-4334.

Connor, E., Mwamuka, J., Gole, A., Murphy, C. \& Wyatt, M. (2005). Gold nanoparticles are taken up by human cells but do not cause acute cytotoxicity. Small, 1, 325-327. 
Corot, C., Robert, P., Idee, J. \& Port, M. (2006). Recent advances in iron oxide nanocrystal technology for medical imaging. Adv Drug Deliv Rev., 58, $1471-1504$.

Costa, P. (2001). An alternative method to the evaluation of similarity factor in dissolution testing. Int. J. Pharm., 220 (1-2), 77-83.

Costa, P. \& Lobo, J. (2001). Modeling and comparison of dissolution profiles. Eur. J. Pharm. Sci., 13, 123-133.

Crank, J. (1975). The Mathematics of Diffusion. Clarendon Press, Oxford.

Cummings, L., Richardson, G. \& Hazelwood, L. (2000). Drug delivery by magnetic microspheres. In: Proc. Mathematics in Medicine Study Group.. UK: University of Notingham, Nottingham, pp 69-83.

Cuong, N.V., Jiang, J.L., Li, Y.L., Chen, J.R., Jwo, S. \& Hsieh, M. (2011). Doxorubicin-loaded peg-pcl-peg micelle using xenograph model of nude mice: Effect of multiple administration of micelle on the suppression of human breast cancer. Cancers, 3, 61-78.

Dash, S., Narasimha, P., Nath, L. \& Chowdhury, P. (2010). Kinetic modeling on drug release from controlled drug delivery systems. Acta Poloniae Pharmaceutica - Drug Research, 67 (3), 217-223.

Day, A. \& Prestwich, G. (2002). Hyaluronan-binding proteins: Tying up the giant. J. Biol. Chem., 277, 4585-4588.

De Jong, W. \& Borm, P. (2008). Drug delivery and nanoparticles: Applications and hazards. International Journal of Nanomedicine, 3(2), 133-149.

Debye, P. (1929). Polar molecules. The Chemical Catalog Company, Inc. 
Decuzzi, P. \& Ferrari, M. (2006). The adhesive strength of non-spherical particles mediated by specific interactions. Biomater, 27, 5307-5314.

Decuzzi, P., Godin, B., Tanaka, T., Lee, S., Chaippini, C., Liu, X. \& FerRARI, J. (2010). Size and shape effects in the biodistribution of intravascularly injected particles. J. Control Release, 141, 320-7.

Delgado, A. \& Arroyo, F. (2002). Electrokinetic Phenomena and Their Experimental Determination: An Overview. (Chapter 1) In: Interfacial Electrokinetics and Electrophoresis.. Marcel Dekker, New York, USA.

Delgado, A., González-Caballero, F., Hunter, R., Koopal, L. \& LyKLEMA, J. (2007). Measurement and interpretation of electrokinetic phenomena. Journal of Colloid and Interface Science, 309, 194-224.

Desai, S., Simonelli, A. \& Higuchi, W. (1965). Investigation of factors influencing release release of solid drug dispersed in inert matrices. J. Pharm. Sci., 54, 1459-1464.

DEY, T. (2006). Polymer-coated magnetic nanoparticles: Surface modification and end-functionalization. J. Nanosci. Nanotechnol., 6, 2479-2483.

Di Marco, M., Port, M., Couvreur, P., Dubernet, C., Ballirano, P. \& SADUN, C. (2006). Structural characterization of ultrasmall superparamagnetic iron oxide (uspio) particles in aqueous suspension by energy dispersive $\mathrm{x}$-ray diffraction (edxd). J. Am. Chem. Soc., 128, 10054-10059.

Dobson, J. (2006a). Gene therapy progress and prospects: magnetic nanoparticlebased gene delivery. Gene Therapy, 13, 283-287.

Dobson, J. (2006b). Magnetic nanoparticles for drug delivery. Drug. Dev. Res., 67, 55-60. 
Du, J.Z., Du, X.., MAO, C.Q. \& WANG, J. (2011). Tailor-made dual ph-sensitive polymer-doxorubicin nanoparticles for efficient anticancer drug delivery. J. Am. Chem. Soc., 133, 17560-17563.

Duran, J., Arias, J., Gallardo, V. \& Delgado, A. (2008). Magnetic colloids as drug vehicles. J. Pharm. Sci., 97, 2948-2983.

Elnakat, H. \& Ratnam, M. (2004). Distribution, functionality and gene regulation of folate receptor isoforms: implications in targeted therapy. Adv. Drug Deliv. Rev., 56, 1067-1084.

Elster, A. \& Burdette, J. (2001). Questions and answers in Magnetic Resonance Imaging. St Louis, USA: Mosby.

Enüstün, B. \& Turkevich, J. (1963). Coagulation of colloidal gold. J. Am. Chem. Soc., 85(21), 3317-3328.

Fauconnier, N., Pons, J., Roger, J. \& Bee, A. (1997). Thiolation of maghemite nanoparticles by dimercaptosuccinic acid. J. Colloid Interface Sci., 194, 427-433.

Feltin, N. \& Pileni, M.P. (1997). New technique for synthesizing iron ferrite magnetic nanosized particles. Langmuir, 13(15), 3927-3933.

Figuerola, A., Di Corato, R. \& Manna, T., L. Pellegrino (2010). From iron oxide nanoparticles towards advanced iron-based inorganic materials designed for biomedical applications. Pharmacological Research, 62, 126-143.

Flesch, C., Unterfinger, Y., Bourgeat-lami, E., Duguet, E., Delaite, C. \& Dumas, P. (2005). Poly(ethylene glycol) surface coated magnetic particles. Macromol. Rapid Commun., 26, 1494-1498.

FlóRez, J. (2008). Farmacología humana. Barcelona: Maason S.A. 
Freitas, M. \& Marchetti, J. (2005). Nimesulide pla microspheres as a potential sustained release system for the treatment of inflammatory diseases. Int. J. Pharm., 295, 201-211.

Freundlich, H. (1926). Colloid and Capillary Chemistry. Methuen and Co., Ltd. London.

Fu, L., Dravid, V. \& Johnson, D. (2001). Self-assembled (sa) bilayer molecular coating on magnetic nanoparticles. Applied Surface Science, 181, 173-178.

GaO, Z., Fain, H. \& RApoport, N. (2005). Controlled and targeted tumor chemotherapy by micellar-encapsulated drug and ultrasound. J Control Release, $102(1), 203-22$.

Ghosh, P., Han, G., De, M., Kim, C. \& Rotello, V. (2008). Gold nanoparticles in delivery applications. Adv. Drug Delivery Rev., 60, 1307-1315.

Goldsmith, J., Randall, N. \& Ross, S. (1978). On methods of expressing dissolution rate data. J. Pharm. Pharm., 30, 347-349.

Govorov, A. \& Richardson, H. (2007). Generating heat with metal nanoparticles. NanoToday, 2, 30-38.

Gratton, S., Ropp, P., Pohlhaus, P., Luft, J., Madden, V., Napier, M. \& DeSimone, J. (2008). The effect of particle design on cellular internalization pathways. Proc. Natl Acad. Sci. USA, 105, 11613-11618.

Greaves, C. (1983). Powder neutron diffraction investigation of vacancy ordering and covalence in gamma-fe2o3. J. Solid State Chem., 49, 325-333.

Gref, R., Minamitake, Y., Peracchia, M., Trubetskoy, V., Torchilin, V. \& LANGer, R. (1994). Biodegradable long-circulating polymeric nanospheres. Science, 263(5153), 1600-3. 
GreIsh, K. (2007). Enhanced permeability and retention of macromolecular drugs in solid tumors: a royal gate for targeted anticancer nanomedicines. J Drug Target., 15, 457-64.

Grosse, C. \& Delagdo, A. (2010). Dielectric dispersion in aqueous colloidal systems. Current Opinion in Colloid and Interface Science, 15 (3), 145-159.

Grosvenor, A.P., Kobe, B.A., Biesinger, M.C. \& McIntyre, N.S. (2004). Investigation of multiplet splitting of fe $2 \mathrm{p}$ xps spectra and bonding in iron compounds. Surf. Interface Anal., 36, 1564-1574.

Gullotti, E. \& Yeo, Y. (2009). Extracellularly activated nanocarriers: a new paradigm of tumor targeted drug delivery. Mol Pharm, 6, 1041-51.

Guo, D., Wu, C., Li, X., Jiang, H., Wang, X. \& Chen, B. (2008). In vitro cellular uptake and cytotoxic effects of functionalized nickel nanoparticles on leukemia cancer cells. J. Nanosci. Nanotechnol., 8, 2301-7.

Gupta, A. \& Curtis, A. (2004). Surface modified superparamagnetic nanoparticles for drug delivery: interaction studies with human fibroblasts in culture. $J$. Mater. Sci. Mater. Med., 15 (4), 493-496.

Gupta, A. \& Gupta, M. (2005). Synthesis and surface engineering of iron oxide nanoparticles for biomedical applications. Biomaterials, 26, 3995-4021.

Gupta, A., Naregalkar, R., Vaidya, V. \& Gupta, M. (2007). Recent advances on surface engineering of magnetic iron oxide nanoparticles and their biomedical applications. Nanomedicine (London), 2, 23-39.

HaAs, C. (1965). Phase transitions in crystals with the spinel structure. J. Phys. Chem. Solids, 26, 1225-1226. 
Halbreich, A., Roger, J., Pons, J., Da Silva, M., Hasmonay, E., Roudier, M., Boynard, M., Sestier, C., Amri, A., Geldwerth, D., Fertil, B., BACRI, J. \& SABOlovic, D. (1997). Magnetic maghemite nanoparticles in: Scientific and clinical applications of magnetic carriers. Plenum Press, New York.

Hergt, R., Andra, W., D’Ambly, C., Hilger, I., Kaiser, W., Richter, U. \& Schmidt, H. (1998). Physical limits of hyperthermia using magnetite fine particles. IEEE Trans. Magn., 34, 3745-54.

HiguCHI, T. (1961). Rate of release of medicaments from ointment bases containing drugs in suspensions. J. Pharm. Sci., 50, 874-875.

Higuchi, T. (1963). Mechanisms of sustained action mediation. theoretical analysis of rate of release of solid drugs dispersed in solid matrices. J. Pharm. Sci., 52, $1145-1149$.

Hillaireau, H. \& Couvreur, P. (2009). Nanocarriers' entry into the cell: relevance to drug delivery. Cell. Mol. Life Sci., 66, 2873-96.

Hixson, A. \& Crowell, J. (1931). Dependence of reaction velocity upon surface and agitation, i.theoretical consideration. Ind. Eng. Chem., 23, 923-931.

Hobson, B. \& Denekamp, J. (1984). Endothelial proliferation in tumours and normal tissues: continuous labelling studies. Br J Cancer., 49, 405-413.

Hu, S., Liu, T., Liu, D. \& Chen, S. (2007). Nano-ferrosponges for controlled drug release. J. Controlled Release, 121, 181-189.

Hunter, R. (1981). Zeta Potential in Colloid Science. Academic Press: New York.

Hunter, R. (1987). Foundations of Colloid Science, Vol.I. Oxford Univ. Press, Oxford. 
Husband, J. \& Reznek, R. (2000). Cancer imaging 2000: principles, strategies, challenges. Cancer Imaging, 1, 1-4.

Hussain, S., Braydich-Stolle, L., Schrand, A., Murdock, R., Yu, K., Mattie, D., Schlager, J. \& Torrones, M. (2009). Toxicity evaluation for safe use of nanomaterials: Recent achievements and technical challenges. Adv. Mater., 21, 1549-1559.

Jain, T., Morales, M., Sahoo, S., Leslie-Pelecky, D. \& Labhasetwar, V. (2005). Iron-oxide nanoparticles for sustained delivery of anticancer agents. Mol Pharm, 2(3), 194-205.

Jaracz, S., Chen, J., Kuznetsova, L. \& Ojima, I. (2005). Recent advances in tumor-targeting anticancer conjugates. Bioorganic $\mathcal{E} 3$ Medicinal Chemistry, 13, 5043-5054.

JeOng, J., HA, T. \& Chung, B. (2006). Enhanced reusability of hexa-argininetagged esterase immobilized on gold-coated magnetic nanoparticles. Anal. Chim. Acta, 569, 203-209.

Jezzard, P., Matthews, P. \& Smith, S. (2002). Functional MRI: an introduction to methods. Oxford Univ. Press: Oxfrod.

Jia, Y., Yuan, M., Yaun, H., Huang, X., Sui, X., Cui, X., Tang, F., Peng, J., Chen, J., Lu, S., Xu, W., Zhang, L. \& Guo, Q. (2012). Coencapsulated of magnetic fe3o4 nanoparticles and doxorubicin into biodegradable plga nanocarriers for intratumoral drug delivery. Int. J. Nanomedicine, $\mathbf{7}$, $1697-1708$.

Jordan, A. (2001). Presentation of a new magnetic field therapy system for the treatment of human solid tumors with magnetic fluid hyperthermia. J. Magn. Magn. Mater., 225, 118-26. 
Jordan, A., Wurst, P., Scholz, R., Tesche, B., Fahling, H., Mitrovics, T., Vogl, T., Cervos-navarro, J. \& Felix, R. (1996). Cellular uptake of magnetic fluid particles and their effects on human adenocarcinoma cells exposed to ac magnetic fields in vitro. Int. J. Hyperthermia, 12, 705-722.

Jordan, A., Scholz, R., Wust, P., Fahling, H., Krause, J., Wlodarczyk, W., Sander, B., Vogl, T. \& Felix, R. (1997). Effects of magnetic fluid hyperthermia (mfh) on c3h mammary carcinoma in vivo. Int. J. Hyperthermia, $13(6), 587-605$.

JUNG, C. (1995). Surface properties of superparamagnetic iron oxide mr contrast agents: ferumoxides, ferumoxtran, ferumoxil. Magn. Reson. Imaging, 13, 675691.

Kachkachi, H., Ezzir, A., Nogues, M. \& Tronc, E. (2000). Surface effects in nanoparticles: application to maghemite gamma- $f e_{2} o_{3}$. Eur. Phys. J. B, 14, $681-689$.

Kellaway, I., ed. (1991). Proceedings and program of the 18th International Symposium on Controlled Release of Bioactive Materials, p. 275. Controlled Release Society in Deerfield IL, USA.

Kelly, K., Coronado, E., Zhao, L. \& Schatz, G. (2003). The optical properties of metal nanoparticles: The influence of size, shape, and dielectric environment. J Phys. Chem. B., 107, 668-677.

Kerbel, R. (2000). Tumor angiogenesis: past, present and the near future. Carcinogenesis, 21, 505-515.

KerKer, M. (1969). The sattering of light and other electromagnetic radiation. (Advanced level. A classic on theory of light scattering). Academic Press: New York. 
Kim, D., Lee, E., Оh, K., Gao, Z. \& Bae, Y. (2008). Doxorubicin-loaded polymeric micelle overcomes multidrug resistance of cancer by double-targetting folate receptor and early endosomal ph. Small, 4 (11), 2043-50.

Kim, J., Yoon, T., Yu, K., Kim, B., Park, S., Kim, H., Lee, K., Park, S., Lee, J. \& Chо, M. (2006). Toxicity and tissue distribution of magnetic nanoparticles in mice. Toxicol. Sci., 89, 338-347.

KIM, S. \& CHU, C.C. (2000). In vitro release bahavior of dextran-methacrylate hydrogels using doxorubicin and other model compounds. J. Biomater Appl, 15, 23-46.

Kim, Y., Park, J. \& Prausnitz, M. (2012). Microneedles for drug and vaccine delivery. Advanced Drug Delivery Reviews, 64, 1547-1568.

Kodama, R., Berkowitz, A., McNiff, E. \& Foner, S. (1996). Surface spin disorder in nife2o4 nanoparticles. Phys. Rev. Lett., 77, 394-397.

Kohane, D. (2007). Microparticles and nanoparticles for drug delivery. Biotechnol. Bioeng, 96, 203-209.

Koleva, D., Guo, Z., van Breugel, K. \& De Wit, J. (2009). The beneficial secondary effects of conventional and pulse cathodic protection for reinforced concrete, evidenced by x-ray and microscopic analysis of the steel surface and the steel/cement paste interface. Mater. Corros., 60(9), 704-715.

Korsmeyer, R., Gurny, R., Doelker, E., Buri, P. \& Peppas, N. (1983). Mechanisms of solute release from porous hydrophilic polymers. Intern. J. Pharm., 15, 25-35.

KREUteR, J. (1983). Evaluation of nanoparticles as drug-delivery systems i: Preparation methods. Pharm. Acta Helv., 58, 196-209. 
Kreuter, J. (1985). Factors influencing the body distribution of polyacrylic nanoparticles. In: Drug Targetting. Elsevier, Amsterdam.

Kumar, A., Sahoo, B., Montpetit, A., Behera, S., Lockey, R. \& S.S., M. (2007). Development of hyaluronic acid-fe2o3 hybrid magnetic nanoparticles for targeted delivery of peptides. Nanomedicine, 3, 132-7.

Kuperman, V. (2000). Magnetic resonance imaging: physical principles and applications. Academic Press: San Diego.

Kwon, J., Hwang, S., Jin, H., Kim, D., Minai-Tehrani, A., Yoon, H., Choi, M., Yoon, T., Han, D., Kang, Y., Yoon, B., Lee, J. \& Cho, M. (2008). Body distribution of inhaled fluorescent magnetic nanoparticles in the mice. J. Occup. Health, 50(1), 1-6.

LAGENBUChER, F. (1972). Linearization of dissolution rate curves by the weibull distribution. J. Pharm. Pharmacol., 24, 979-981.

Lang, C., Schüler, D. \& Faivre, D. (2007). Synthesis of magnetite nanoparticles for bio-and nanotechnology: genetic engineering and biomimetics of bacterial magnetosomes. Macromol. Biosci., 7, 144-51.

Langmuir, I. (1916). The constitution and fundamental properties of solids and liquids. part i: Solids. J. Am. Chem. Soc., 38 (11), 2221-2295.

LANGMUIR, I. (1918). The adsorption of gases on plane surfaces of glass, mica and platinum. J. Amer. Chem. Soc., 40, 1361-1368.

LApidus, H. \& LoRdi, N. (1966). Some factors affecting the release of a watersoluble drug from a compresed hydrophilic matrix. J. Pharm. Sci., 55, 840-843.

Laurent, S., Forge, D., Port, M., null Roch, A., Robic, C., Elst, L. \& MüLleR, R. (2008). Magnetic iron oxide nanoparticles: Synthesis, stabiliza- 
tion, vectorization, physicochemical characterizations, and biological applications. Chem. Rev., 108, 2064-2110.

Laurent, S., Burtea, C., Thirifays, C., Häfeli, U. \& Mahmoudi, M. (2011). Crucial ignored parameters on nanotoxicology: The importance of toxicity assay modifications and cell vision. PLoS ONE, 7, e29997.

Lecommandoux, S., Sandre, O., Chécot, F. \& Perzynski, R. (2006). Smart hybrid magnetic self-assembled micelles and hollow capsules. Prog. Solid State Chem., 34, 171-179.

Lee, J., Lu, J., Low, P. \& Fuchs, P. (2002). Synthesis and evaluation of taxol-folic acid conjugates as targeted antineoplastics. Bioorg. Med. Chem., 10, $2397-2414$.

LEE, P. (2011). Modeling of drug release from matrix systems involving moving boundbound: approximate analytical solutions. Int. J. Pharm., 418 (1), 18-27.

Lee, S., Bae, S., Takemura, Y., Shim, I., Kim, T., Kim, J., Lee, H., Zurn, S. \& KIM, C. (2007). Self-heating characteristics of cobalt ferrite nanoparticles for hyperthermia application. J. Magn. Magn. Mater., 310, 2868-2870.

Lewinski, N., Colvin, V. \& Drezek, R. (2008). Cytotoxity of nanoparticles. Small, 4, 26-49.

Li, L., Fan, M., Brown, R., van Leeuwen, J., Wang, J., Wang, W., Song, Y. \& ZhANG, P. (2006). Synthesis, properties, and environmental applications of nanoscale iron-based materials: a review. Critical Rev Environ Sci Technol, 26, 405-31.

Liao, M. \& Chen, D. (2002). Preparation and charaterization of a novel magnetic nano-adsorbent. J. Mater. Chem., 12, 3654-3659. 
Lin, B., Shen, X. \& Cui, S. (2007). Application of nanosized fe3o4 in anticancer drug carriers with target-orientation and sustained-release properties. Biomed. Mater., 2, 132-134.

Liu, C., Rondinone, A. \& Zhang, Z. (2000). Synthesis of magnetic spinel ferrite cofe2o4 nanoparticles from ferric salt and characterization of the size-dependent superparamagnetic properties. Pure Appl. Chem., 72, 37-45.

Liv, H., Chen, D., Li, L., Liu, T., Tan, L., Wu, I. \& Tang, F. (2011). Multifunctional gold nanoshells on silica nanorattles: A platform for the combination of photothermal therapy and chemotherapy with low systemic toxicity. Angew. Chem. Int. Ed., 50, 891-895.

Liu, T., Hu, S., Liu, K., Liu, D. \& Chen, S. (2008). Study on controlled drug permeation of magnetic-sensitive ferrogels: effect of fe3o4 and pva. J. Control. Release, 126(3), 228-36.

Liu, X., Kamiski, M., Chen, H., Torno, M., Taylor, L. \& Rosengart, A. (2007a). Synthesis and characterization of highly-magnetic biodegradable poly(d,l-lactide-co- glycolide) nanospheres. J Control Release, 119, 52-8.

LiU, X., YAnG, G. \& Fu, S. (2007b). Mass synthesis of nanocrystalline spinel ferrites by a polymer-pyrolysis route. Mater. Sci. Eng. C, 27, 750-755.

Lopez-Viota, J., Mandal, S., Delgado, A., Toca-Herrera, J., Moller, M., Zanuttin, F., Balestrino, M. \& Krol, S. (2009). Electrophoretic characterization of gold nanoparticles functionalized with human serum albumin (hsa) and creatine. J. Colloid Interface Sci., 1, 215-223.

Lu, A., Salabas, E. \& Schüth, F. (2007). Magnetic nanoparticles: synthesis, protection, functionalization, and application. Angew. Chem., Int. Ed., 46, $1222-44$. 
Lu, Y., Yin, Y., Mayers, B. \& XIA, Y. (2002). Modifying the surface properties of superparamagnetic iron oxide nanoparticles through a sol-gel approach. Nano Lett., 2, 183-186.

Lübbe, A., Bergemann, C., Huhnt, W., Fricke, T., Riess, H., Brock, J. \& Hunn, D. (1996). Preclinical experiences with magnetic drug targetting: tolerance and efficacy. Cancer Res, 56, 4694-701.

Lübbe, A., Bergemann, C., Brock, J. \& McClure, D. (1999). Physiological aspects in magnetic drug-targeting. J. Magn. Magn. Mater., 194, 149-55.

Lutz, J.F., Stiller, S., Hoth, A., Kaufner, L., Pison, U. \& Cartier, R. (2006). One-pot synthesis of pegylated ultrasmall iron-oxide nanoparticles and their in vivo evaluation as magnetic resonance imaging contrast agents. Biomacromolecules, 7, 3132-8.

Lyer, A., Khaled, G., Fang, J. \& Maeda, H. (2006). Exploiting the enhanced permeability and retention effect for tumor targeting. Drug Discov Today, 11, $812-818$.

Lyklema, J. (1991). Electrified interfaces in aqueous dispersions of solids. Pure Appl. Chem., 63, 895-906.

Ma, D., Guan, J., Normandin, F., Dénommée, S., Enright, G., Veres, T. \& Simard, B. (2006). Multifunctional nano-architecture for biomedical applications. Chem. Mater., 18, 1920-27.

MAEDA, H. (2001). The enhanced permeability and retention (epr) effect in tumor vasculature: the key role of tumor-selective macromolecular drug targeting. Adv Enzyme Regul., 41, 189-207. 
Maeda, H. (2010). Tumor-selective delivery of macromolecular drugs via the epr effect: background and future prospects. Bioconjug Chem, 21(5), 797-802.

Maeda, H., Matsumura, Y., Oda, T. \& Sasamoto, K. (1986). In :Protein tailoring for food and medical uses. p.353-382. Marcel Dekker, New York, USA.

Maeda, H., Bharate, G. \& Daruwalla, J. (2009). Polymeric drugs for efficient tumor-targeted drug delivery based on epr-effect. Eur J Pharm Biopharm., 71, 409-19.

Mahmoudi, M., Simchi, A., Imani, M., Shokrgozar, M., Milani, A., Häfeli, U. \& Stroeve, P. (2010). A new approach for the in vitro indentification of the cytotoxicity of superparamagnetic iron oxide nanoparticles. Colloids Surf B Biointerfaces, 75, 300-309.

Mahmoudi, M., Laurent, S., Shokrgozar, M. \& Hosseinkhani, M. (2011a). Toxicity evaluations of superparamagnetic iron oxide nanoparticles: Cell vision versus physicochemical properties of nanoparticles. ACS Nano, 5, 7263-7276.

Mahmoudi, M., Sant, S., Wang, S., Laurent, S. \& Sen, T. (2011b). Superparamagnetic iron oxide nanoparticles (spions): development, surface modification and applications in chemotherapy. Adv Drug Deliv Rev, 63 (1-2), 24-46.

Mahoney, B., Raghunand, N., Baggett, B. \& Gillies, R. (2003). Tumor acidity, ion trapping and chemotherapeutics. 1. acid ph affects the distribution of chemotherapeutic agents in vitro. Biochemical Pharmacology, 66, 1207-1218.

Martinez, L., Leinen, D., Martin, F., Gabas, M., Ramos-Barrado, J.R., Quagliata, E. \& Dalchiele, E.A. (2007). Electrochemical growth of diverse iron oxide (fe3o4, a-feooh, and c-feooh) thin films by electrodeposition chemical tuning. J. Electrochem. Soc., 154(3), D126-D133. 
Massart, R. (1981). Preparation of aqueous magnetic liquids in alkaline and acidic media. IEEE Trans. Magn., MAG-17, 1247-1248.

Massen, S., Fattal, E., Müller, R.H. \& Couvreur, P. (1993). Cell cultures for the assessment of toxicity and uptake of polymeric particulate drug carriers. S.T.P. Pharma Sci., 3(1), 11-22.

Matsumura, Y. (2008). Poly (amino acid) micelle nanocarriers in preclinical and clinical studies. Adv. Drug Deliv. Rev., 60, 899-914.

Mauger, J., Chilko, D. \& Howard, S. (1986). On the analysis of the dissolution data. Drug Dev. Ind. Pharm., 12, 969-992.

Maurer-Jones, M., Bantz, K., Love, S., Marquis, B. \& Haynes, C. (2009). Toxicity of therapeutic nanoparticles. Nanomedicine, 4, 219-241.

McBain, S., Yiu, H. \& Dobson, J. (2008). Magnetic nanoparticles for gene and drug delivery. Int. J. Nanomedicine, 3(2), 169-180.

Mikhaylova, M., Jo, Y., Kim, D., Bobrysheva, N., Andersson, Y., Eriksson, T., Osmolowsky, M., Semenov, V. \& Muhammed, M. (2004). The effect of biocompatible coating layers on magnetic properties of superparamagnetic iron oxide nanoparticles. Hyperfine Interact., 156-157, 257-263.

Millan, A., Urtizberea, A., Silva, N., Palacio, F., Amaral, V., Snoeck, E. \& Serin, V. (2007). Surface effects in maghemite nanoparticles. J. Magn. Magn. Mater., 312, L5-L9.

Mills, N., Tornqvist, H., Robinson, S., Gonzalez, M., Darnley, K., Macnee, W., Boon, N., Donaldson, K., Blomberg, A., Sandstrom, T. \& Newby, D. (2005). Diesel exhaust inhalation causes vascular dysfunction and impaired endogenous fibrinolysis. Circulation, 112, 3930-36. 
Minotti, G., Menna, P., Salvatorelli, E., Cairo, G. \& Gianni, L. (2004). Anthracycylines: molecular advances and pharmacologic developments in antitumor activity and cardiotoxicity. Pharmacol. Rev., 56, 185-229.

Mitragotri, S. \& Lahann, J. (2009). Physical approaches to biomaterial design. Nat Mater., 8(1), 15-23.

Moghimi, M., Hunter, A. \& Murray, J. (2001). Long-circulating and targetspecific nanoparticles: theory to practice. Pharmacol. Rev., 53, 283-318.

Moore, J. \& Flanner, H. (1996). Mathematical comparison of dissolution profiles. Pharm. Technol., 20, 64-74.

Morales, M.P., Pecharroman, C., Gonzáles Carreño, T. \& Serna, C.J. (1994). Structural characteristics of uniform gamma-fe2o3 particles with different axial (length/width) ratios. J. Solid State Chem., 108, 158-163.

Moroz, P., Jones, S. \& Gray, B. (2002). Magnetically mediated hypethermia: current status and future directions. Int. J. Hyperthermia, 18, 267-84.

Mosbach, K. \& Schröder, U. (1979). Preparation and application of magnetic polymers for targeting of drugs. FEBS Lett., 102, 112-6.

Müller, R., Luck, M., Harnisch, S. \& Thode, K. (1997). Intravenously injected particles: Properties and interactions with blood proteins - The key determining the organ distribution. In: Scientific and Clinical Applications of Magnetic Carriers. Plenum Press, New York.

Muro, S., Garnacho, C., Champion, J., Leferovich, J., Gajewski, C., Schuchman, E., Mitragotri, S. \& Muzykantov, V. (2008). Control of endothelial targeting and intracellular delivery of therapeutic enzymes by mod- 
ulating the size and shape of icam-1-targeted carriers. Mol. Ther., 16, 14501458.

Nagahara, A., Merrill, D., Coppola, G., Tsukada, S., Schroeder, B., Shaked, G., Wang, L., Blesch, A., Kim, A., Conner, J., Rockenstein, E., Chao, M., Koo, E., Geschwind, D., Masliah, E., Chiba, A. \& Tuszynski, M. (2009). Neuroprotective effects of brain-derived neurotrophic factor in rodent and primate models of alzheimer's disease. Nature Medicine, 15, 331-337.

Najdek, K., Bianconi, F., Da Rocha, T., Zanchet, D., Bonacin, J., Novak, M., Vaz, M. \& Winnischofer, H. (2011). Structure and morphology of spinel $\mathrm{mfe}_{2} \mathrm{O}_{4}(\mathrm{~m}=\mathrm{fe}, \mathrm{co}, \mathrm{ni})$ nanoparticles chemically synthesized from heterometallic complexes. J. Colloid Interface Sci., 358, 39-46.

Nakamura, T., Akita, H., Yamada, Y., Hatakeyama, H. \& Harashima, H. (2012). A multifunctional envelope-type nanodevice for use in nanomedicine: Concept and applications. Accounts of Chemical Research, 45 (7), 1113-1121.

Nawara, K., Romiszewski, J., Kijewska, K., Szczytko, J., Twardowski, A., Mazur, M. \& Krysinski, P. (2012). Adsorption of doxorubicin onto citrate-stabilized magnetic nanoparticles. J. Phys. Chem. C, 116(9), 55985609.

Neuberger, T., Schopf, B., Hotmann, H. \& Hofmann, B., M. von ReChenberg (2005). Superparamagnetic nanoparticles for biomedical applications: Possibilities and limitations of a new drug delivery system. J. Magn. Magn. Mater., 293, 483-496.

Niu, G., Coqburn, B. \& Hughes, J. (2010). Preparation and characterization of doxorubicin liposomes. Methods Mol Biol., 624, 211-9. 
Nowicka, A., Kowalczyk, A., Donten, M., Krysinski, P. \& Stojek, Z. (2009). Influence of a magnetic nanoparticles as a drug carrier on the activity of anticancer drugs: Iinteraction of double stranded dna and doxorubicin modified with a carrier. Anal. Chem., 81, 7474-7483.

Oberdörster, G. (2009). Safety assessment foe nanotechnology and nanomedicine: concepts of nanotoxicology. Journal of Internal Medicine, 267, 89-105.

Oberdörster, G., Oberdörster, E. \& Oberdörster, J. (2005). Nanotoxicology: an emerging discipline evolving from studies of ultrafine particles. Env. Health Perspect., 113, 823-39.

Oberdörster, G., Stone, V. \& Donaldson, K. (2007). Toxicology of nanoparticles: a historical perspective. Nanotoxicology, 1, 2-25.

O'Brien, R. \& White, L. (1978). Electrophoretic mobility of a spherical colloidal particle. J. Chem. Soc. Faraday Trans. II, 74, 1607-1626.

Ohshima, H. (2002). In: Interfacial Electrokinetics and Electrophoresis. Marcel Dekker, New York.

Ohtsubo, T., Igawa, H., Saito, T., Matsumoto, H., Park, H., Song, C., KANO, E. \& SAITO, H. (2001). Acacid environment modifies heat- or radiationinduced apoptosis in human maxillary cancer cells. Int. J. Radiat Oncol Biol Phys, 49, 1391-8.

Paciotti, G., Kingston, D. \& Tamarkin, L. (2006). Colloidal gold nanoparticles: A novel nanoparticle platform for developing multifunctional tumortargeted drug delivery vectors. Drug Dev. Res., 67, 47-54. 
Pakunlu, R., Wang, Y., Saad, M., Khandare, J., Starovoytov, V. \& Minko, T. (2006). In vitro and in vivo intracellular liposomal delivery of antisense oligonucleotides and anticancer drug. J Controlled Release, 114 (2), $153-62$.

Pankhurst, Q., Connolly, J., Jones, S. \& Dobson, J. (2003). Applications of magnetic nanoparticles in biomedicine. J. Phys. D: Appl. Phys., 36, R167R181.

Park, H., Yang, J., Lee, J., HaAm, S., Choi, I. \& Yoo, K. (2009). Multifunctional nanoparticles combined doxorubicin and photothermal treatments. ACS Nano, 3 (10), 2919-2926.

Parveen, S., Misra, R. \& Sahoo, S. (2012). Nanoparticles: a boon to drug delivery, therapeutics, diagnostics and imaging. Nanomedicine: Nanotechnology, Biology, and Medicine, 8, 147-166.

Patil, R., Guhagarkar, S. \& Devarajan, P. (2008). Engineered nanocarriers of doxorubicin: a current update. Crit. Rev. Ther. Drug Carrier Syst., 25, 1-61.

Pecora, R. (1985). Dynamic light scattering: Applications of Photon Correlation Spectroscopy.. Plenum, New York.

Peira, E., Marzola, P., Podio, V., Aime, S., Sbarbati, A. \& Gasco, M. (2003). In vitro and in vivo study of solid lipid nanoparticles loaded with superparamagnetic iron oxide. J. Drug Target., 11, 19-24.

Pelley, J., DaAr, A. \& Saner, M. (2009). State of academic knowledge on toxicity and biological fate of quantum dots. Toxicol Sci., 112, 276-96.

Peppas, N. (1985). Analysis of fickian and non-fickian drug release from polymers. Pharm. Acta Helv., 60, 110-111. 
Pham, T., Jackson, J., Halas, N. \& Lee, T. (2002). Preparation and characterization of gold nanoshells coated with self-assembled monolayers. Langmuir, 18, 4915-4920.

Pilloni, M., Nicolas, J., Marsaud, V., Bouchemal, K., Frongia, F., Scano, A., Ennas, G. \& Dubernet, C. (2010). Pegylation and preliminary biocompatibility evaluation of magnetite-silica nanocomposites obtained by high energy ball milling. Int. J. Pharm., 401, 103-112.

Plank, M. \& Sleeman, B. (2003). Tumour-induced angiogenesis: A review. Journal of Theoretical Medicine, 5, 137-153.

Polli, J., Rekhi, G., Augsburger, L. \& Shah, V. (1997). Methods to compare dissolution profiles and a rationale for wide dissolution specifications for metoprolol tartrate tablets. J. Pharm. Sci., 86 (6), 690-700.

Prados, J., Melquizo, C., Ortiz, R., Velez, C., Alvarez, P., Arias, M., J.L. Ruiz, Gallardo, V. \& Aranega, A. (2012). Doxorubicin-loaded nanoparticles: new advances in breast cancer therapy. Anticancer Agents Med Chem, 12(9), 1058-70.

Prasad, P., ed. (2006). Magnetic Resonance Imaging: Methods and Biologic Applications.. Humana Press, New Jersey.

Prashant, K., El-Sayed, I. \& El-Sayed, M. (2007). Au nanoparticles target cancer. NanoToday, 2, 18-29.

PriJic, S. \& Sersa, G. (2011). Magnetic nanoparticles as targeted delivery systems in oncology. Radiol Oncol, 45(1), 1-16. 
Prime, K. \& Whitesides, G. (1991). Self-assembled organic monolayers: model systems for studying adsorption of proteins at surfaces. Science, 252, 11641167.

Prista, L., Alves, A. \& Morgado, R. (1995). Técnica Farmacêutica e Farmácia Galénica, Vol. I. Fundacão Calouste Gulbenkian, Lisboa.

Raghunand, N. \& Gillies, R. (2002). ph and drug resistance in tumors. Drug Resist Updates, 3, 39-47.

Ravi Kumar, M., Gameti, M. \& Mohapatra, S. (2004). Cationic silica nanoparticles as gene carriers: synthesis, characterization and transfection efficiency in vitro and in vivo. J. Nanosci. Naotechnol., 4, 876-81.

Reddy, L., Arias, J., Nicolas, J. \& Couvreur, P. (2012). Magnetic nanoparticles: Design and characterization, toxicity and biocompatibility, pharmaceutical and biomedical applications. Chem. Rev., 112 (11), 5818-5878.

ReILly, J. (1992). Principles of nerve and heart excitation by time-varying magnetic fields. Ann. New York Acad. Sci., 649, 96-117.

Rifkin, R., Gregory, S., Mohrbacher, M. \& Hussein, M. (2006). Pegylated liposomal doxorubicin, vincristine, and dexamethasone provide significant reduction in toxicity compared with doxorubicin, vincristine, and dexamethasone in patients with newly diagnosed multiple myeloma. Cancer, 106 (4), $848-858$.

Ritger, P. \& Peppas, N. (1987a). A simple equation for description of solute release. i. fickian and non-fickian release from non-swellable devices in the form of slabs, spheres, cylinders or discs. J. Control Release, 5, 23-36. 
Ritger, P. \& Peppas, N. (1987b). A simple equation for description of solute release. ii. fickian and anomalous release from swellable devices. J. Controlled Release, 5, 37-42.

Roseman, T. \& Higuchi, W. (1970). Release of medroxyprogesterona acetate from a silicone polymer. J. Pharm. Sci., 59, 353-357.

Rosensweig, R. (2002). Heating magnetic fluid with alternating magnetic field. J. Magn. Magn. Mater., 252, 370-74.

Rudzka, K., Delgado, A. \& L. Viota, J. (2012). Maghemite functionalization for antitumor drug vehiculization. Molecular Pharmaceutics, 9 (7), 2017-2028.

Salgueiriño Maceira, V., Correa-Duarte, M.A., Farle, M., LopezQuintela, A., Sieradzki, K. \& Diaz, R. (2006). Bifunctional gold-coated magnetic silica spheres. Chem. Mater., 18, 2701-2706.

Sandhu, A., Handa, H. \& ABe, M. (2010). Synthesis and applications of magnetic nanoparticles for biorecognition and point of care medical diagnostics. Nanotechnology, 21(44), 442001-442022.

Sarin, H., Kanevsky, A., Wu, H., Brimacombe, K., Fung, S., Sousa, A., Auh, S., Wilson, C., Sharma, K., Aronova, M., Leapman, R., GrifFITHS, G. \& HALL, M. (2008). Effective transvascular delivery of nanoparticles across the blood-brain tumor barrier into malignant glioma cells. J Transl Med, 6, 80 .

Schlorf, T., Meincke, M., Kossel, C.C., E.and Glüer, Jansen, O. \& Mentlein, R. (2011). Biological properties of iron oxide nanoparticles for cellular and molecular magnetic resonance imaging. Int. J. Mol. Sci., 12, 12-23. 
Schmidt, R. \& Thews, G. (1995). Physiologie des Menschen, p.515. Springer, Berlin.

Schroeder, U., Sommerfeld, P., Ulrich, S. \& Sabel, B. (1998). Nanoparticle technology for delivery of drugs across the blood-brain barrier. J. Pharm. Sci., 87, 1305-1307.

Schulze, E., Ferruci, J., Poss, K., Lapointe, L., Bogdanova, A. \& WEISSLEDER, R. (1995). Cellular uptake and trafficking of a prototypical magnetic iron oxide label in vitro. Invest. Radiol., 30, 604-610.

Schütt, W., Grüttner, C., U.O., H., Zborowski, M., Teller, J., Putzar, H. \& Schümichen, C. (1997). Applications of magnetic targeting in diagnosis and therapy - possibilities and limitations: A mini-review. Hybridoma, 16(1), 109-117.

Senter, P. \& Sievers, E. (2012). The discovery and development of brentuximab vedotin for use in relapsed hodgkin lymphoma and systemic anaplastic large cell lymphoma. Nature Biotechnology, 30 (7), 631-637.

Senyei, A., Widder, K. \& Czerlinski, C. (1978). Magnetic guidance of drug carrying microspheres. J. Appl. Phys., 49, 3578-3583.

Seo, W., Lee, J., Sun, X., Suzuki, Y., Mann, D., Liu, Z., Terashima, M., Yang, P., McConnell, M., Nishimura, D. \& Dai, H. (2006). Feco/graphitic-shell nanocrystals as advanced magnetic-resonance-imaging and near-infrared agents. Nat. Mater., 5, 971-6.

Shah, V., Lesko, L., Fan, J., Fleischer, N., Handerson, J., Malinowski, H., Makary, M., Ouderkirk, L., Roy, S., Sathe, P., Singh, G., TillMan, L., Tsong, Y. \& Williams, R. (1997). Fda guidance for industry: disso- 
lution testing of immediate release solid oral dosage forms. Dissolution Technol., $4,15-22$.

Sharifi, S., Behzadi, S., Laurent, S., Forrest, L., Stroeve, P. \& MahMoudi, M. (2012). Toxicity of nanomaterials. Chem. Soc. Rev., 41, 2323-2343.

Shmakov, A.N., Kryukova, G.N., Tsybhulya, V.S., Chiuviliu, A.L. \& Solovyeva, V.P. (1995). Vacancy ordering in gamma-fe2o3: Synchrotron xray powder diffraction and high-resolution electron microscopy studies. J. Appl. Crystallogr., 28, 141-145.

Siegbahn, K. (1967). ESCA: Molecular and Solid State Structure studied by means of Electron Spectroscopy.. Uppsåla.

Siepmann, J. \& Peppas, N. (2001). Modeling of drug release from delivery systems based on hydroxypropyl methylcellulose (hpmc) . Adv. Drug Deliv. Rev., 64, 139-174.

Singh, N., Jenkins, G., Asadi, R. \& Doak, S. (2010). Potential toxicity of superparamagnetic iron oxide nanoparticles (spion). Nano Reviews, 1, 5358-.

Skoog, D., Holler, F. \& Nieman, T. (1998). Principles of instrumental analysis, 5th Ed.. New York: Thomson Brooks/Cole.

Slowing, I., Trewyn, B., Giri, S. \& Lin, V. (2007). Mesoporous silica nanoparticles for drug delivery and biosensing applications. Adv. Funct. Mater., 17, $1225-1236$.

Slowing, I., Vivero-Escoto, J., Wu, C.W. \& Lin, V.Y. (2008). Mesoporous silica nanoparticles as controlled release drug delivery and gene transfection carriers. Adv. Drug Delivery Rev., 60, 1278-1288. 
Soenen, S., Himmelreich, U., Nuytten, N., Pisanic 2nd, T., Ferrari, A. \& De Cuyper, M. (2010). Intracellular nanoparticle coating stability determines nanoparticle diagnostics effecicacy and cell functionality. Small, $\mathbf{6}$, $2136-45$.

Soenen, S., Rivera-Gil, P., Montenegro, J., Parak, W., De Smedt, S. \& Braeckmans, K. (2011). Cellular toxicity of inorganic nanoparticles: Common aspects and guidelines for improved nanotoxicity evaluation. Nano Today, 6 , $446-465$.

Sonvico, F., Mornet, S., Vasseur, S., Dubernet, C., Jalllard, D., Degrouard, J., Hoebeke, J., Duguet, E., Colombo, P. \& Couvreur, P. (2005). Folate-conjugated iron oxide nanoparticles for solid tumor targeting as potential specific magnetic hyperthermia mediators: synthesis, physicochemical characterization, and in vitro experiments. Bioconjug. Chem., 16 (5), 11811188.

StÖвеR, W., Fink, A. \& Bohr, E. (1968). Controlled growth of monodisperse silica spheres in the micron size range. J. Colliod Interface Sci., 26, 62-69.

Sturgeon, R. \& Schulman, S. (1977). Electronic absorption spectra and protolytic equilibria of doxorubicin: Direct spectrophotometric determination of microconstants. J Pharm. Sci., 66, 958-961.

Sun, C., Lee, J. \& Zhang, M. (2008). Magnetic nanoparticles in mr imaging and drug delivery. Adv. Drug Delivery Rev., 60, 1252-1265.

Sun, S., Zeng, H., Robinson, D., Raoux, S., Rice, P., Wang, S. \& Li, G. (2004). Monodisperse $m \mathrm{me}_{2} \mathrm{o}_{4}(\mathrm{~m}=\mathrm{fe}, \mathrm{co}, \mathrm{mn})$ nanoparticles. J. Am. Chem. Soc., 126, 273-279. 
Tapeinos, C., Efthimiadou, E., Boukos, N., Charitidis, C., Koklioti, M. \& Kordas, G. (2013). Microspheres as therapeutic delivery agents: synthesis and biological evaluation of ph respresponsive. J Mater. Chem. B, 1, 194-203.

Tartaj, P., Del Puerto Morales, M., Veintemillas-Verdaguer, S., Gonzáles Carreño, T. \& Serna, C.J. (2003). The preparation of magnetic nanoparticles for applications in biomedicine. J. Phys. D: Appl. Phys., 36, R182-R197.

Temesghen, W. \& Sherwood, P. (2002). Analytical utility of valence band x-ray photoelectron spectroscopy of iron and its oxides, with spectral interpretation by cluster and band structure calculations. Anal. Bioanal. Chem., 373, 601-608.

Thassu, D., Deleers, M. \& Pathak, Y., eds. (2007). Nanoparticulate drug delivery systems in: Drugs and the pharmaceutical science; vol. 166. Informa Healthcare USA, Inc.

Thode, K., Müller, R. \& Kresse, M. (2000). Two-time window and multiangle photon correlation spectroscopy size and zeta potential analysis-highly sensitive rapid assay for dispersion stability. J. Pharm. Sci., 89(10), 1317-24.

Thoeny, H., Triantafyllou, M., Birkhauser, F., Froenlich, J., TsherING, D. \& Binser, T.E.A. (2009). Combined ultrasmall superparamagnetic particles of iron oxide-ehnaced and diffusion-weighted magnetic resonance imaging reliably detect pelvic lymph node metastases in normal-sized nodes of bladder and prostate cancer patients. Eur Urol, 55, 761-9.

Tong, R., Yala, L., Fan, T. \& Cheng, J. (2010). The formulation of aptamercoated paclitaxel-polyactide nanoconjugates and their targeting to cancer cells. Biomaterials, 31, 3043-53. 
Toole, B. (1982). In: Cell Biology of the Extracellular Matrix. p.259-294. Plenum Press, New York.

Toole, B., Wright, T. \& Tammi, M. (2002). Hyaluronan-cell interactions in cancer and vascular disease. J. Biol. Chem., 277, 4593-6.

Turley, E., Belch, A., Poppema, S. \& Pilarski, L. (1993). Expression and function of a receptor for hyaluronan-mediated motility on normal and malignant b lymphocytes. Blood, 81, 446-53.

Urbain, O. \& Steman, W. (1941). Magnetic flocculation for removing suspended matter from whey, distillery and east plant wastes, straw board-mill wastes etc.

Vaage, J., Mayhew, E., Lasic, D. \& Martin, F. (1992). Therapy of primary and metastatic mouse mammary carcinomas with doxorubicin encapsulated in long circulating liposomes. Int. J. Cancer, 51, 942-8.

Valenzuela, M., Núñez, M., Villalobos, M., Siles, E., Olea, N., PeDraza, V., McMillan, T. \& Ruíz de Almodóvar, J. (1995). Relationship between doxorubicin cell sensitivity, drug-induced dna double-strand breaks, glutathione content and p-glycoprotein in mammalian tumor cells. Anticancer drugs, 6(6), 749-757.

VAN Oss, C. (2006). Interfacial forces in aqueous media. 2nd ed.; CRC Press: Boca Raton, Florida, USA.

van Oss, C., Chaudhury, M. \& Good, R. (1988). Interfacial liftshift-van der waals and polar interactions in macroscopic system. Chem. Rev., 88, 927-941.

Varallyay, P., Nesbit, G., Muldoon, L., Nixson, R., Delashaw, J., Cohen, J., Petrillo, D., Rink \& Neuwelt, E. (2002). Comparison of two 
superparamagnetic viral-sized iron oxide particles ferumoxides and ferumoxtran10 with a gadolinium chelate in imaging intracranial tumors. Am J Neuroradiol., 23, 510-519.

Viota, J., Durán, J., González-Caballero, F. \& Delgado, A. (2007). Magnetic properties of extremely bimodal magnetite suspensions. J. Magn. Magn. Mater., 314, 80-86.

Viota, J., Rudzka, K., Trueba, A., Torres-Aleman, I. \& Delgado, A. (2011). Electrophoretic characterization of insulin growth factor (igf-1) functionalized magnetic nanoparticles. Langmuir, 27 (10), 6426-32.

Viota, J., Carazo, A., Munoz-Gamez, J., Rudzka, K., GómezSotomayor, R., Ruiz-Extremera, A., Salmerón, J. \& Delgado, A. (2013). Functionalized magnetic nanoparticles as vehicles for the delivery of the antitumor drug gemcitabine to tumor cells. physicochemical in vitro evaluation. Materials Science and Engineering C, 33 (3), 1183-1192.

Vlatrdingerbroek, M. \& den Boer, J., eds. (2003). Magnetic Resonance Imaging: Theory and Practice. 3rd Ed.. Springer, Berlin.

Voltairas, P., Fotiadis, D. \& Michalis, L. (2002). Hydrodynamics of magnetic drug targeting. J. Biomech., 35, 813-21.

Vudathala, G. \& Rogers, J. (1992). Dissolution of fludrocortisone from phospholipid coprecipitates. J. Pharm. Sci., 82, 282-286.

WANG, X. (2007). The application of fe3o4 nanoparticles in cancer research: a new strategy to inhibit drug resistance. J Biomed. Mater. Res. A, 80, 852-60. 
Wang, Y., Hussain, S. \& Krestin, G. (2001). Superparamagnetic iron oxide contrast agents: physicochemical characteristics and applications in mr imaging. Eur Radiol, 11, 2319-31.

Weissleder, R., Bogdanov, A., Neuwelt, E. \& Papisov, M. (1995). Longcirculating iron oxides for $\mathrm{mr}$ imaging. Adv. Drug Delivery Rev., 16 (2-3), $321-334$.

Weitman, S., Lark, R., Coney, L., Fort, D., Frasca, V., Zurawski, V. \& KAMEN, B. (1992). Distribution of the folate receptor gp38 in normal and malignant cell lines and tissues. Cancer Res., 52, 3396-401.

Wells, I.S.I.C.O.U.P.L.., A.F. (1975). Structural Inorganic Chemistry. Oxford Univ. Press, London.

Widder, K., Senyei, A. \& Scapelli, D. (1978). Magnetic microspheres: a model system for site specific drug delivery in vivo. Proc. Soc. Exp. Biol. Med., 58, 141-146.

Widder, K., Senyei, A. \& Ranney, D. (1979). Magnetically responsive microspheres and other carriers for the biophysical targeting of antitumour agents. Adv. Pharmacol. Chemother., 16, 213-271.

Widder, K., Morris, R., Poore, G., Howard, D. \& Senyei, A. (1983). Selective targeting of magnetic albumin microspheres containing low-dose doxorubicin - total remission in yoshida sarcoma-bearing rats. Eur. J. Cancer. Clin. Oncol., 19, 135-9.

Wiessleder, R., Reimer, P., Lee, A., Wittenberg, J. \& Brandy, T. (1990). Mr receptor imaging: ultrasmall iron oxide particles targeted to asialoglycoprotein receptors. AJR Am J Roentgenol., 155(6), 1161-7. 
Williams, P., Carpino, F. \& Zborowski, M. (2009). Magnetic nanoparticle drug carriers and their study by quadrupole magnetic field-flow fractionation. Mol. Pharmaceutics, 6, 1290-1306.

World Health Organization, W. (2008). World cancer report. Tech. rep., International Agency for Research on Cancer (Eds. Peter Boyle Bernard Levin).

YANG, B., Zhang, L. \& Turley, E. (1993). Identification of two hyaluronanbinding domains in the hyaluronan receptor rhamm. J. Biol. Chem., 268, 861723.

Yiu, H., Wright, P. \& Botting, N. (2001). Enzyme immobilisation using sba-15 mesoporous molecular sieves with functionalised surfaces. J Mol Catal B Enzym, 15, 81-92.

You, J., Li, X., De Cui, F., Du, Y., Yuan, H. \& Hu, F. (2008). Folateconjugated polymer micelles for active targeting to cancer cells: preparation, in vitro evaluation of targeting ability and cytotoxicity. Nanotechnology, 19 (4), $1-9$.

Yu, H., Chen, M., Rice, P., Wang, S., White, R. \& Sun, S. (2005). Dumbbell-like bifunctional au-fe3o4 nanoparticles. Nano Lett., 5, 379-82.

YU, S. \& CHow, G. (2004). Carboxyl group (-co2h) functionalized ferrimagnetic iron oxide nanoparticles for potential bio-applications. J. Mater. Chem., 14, $2781-86$.

Zeldowitsch, J. (1934). Adsorption site energy distribution. Acta Physicochim. URSS, 1, 961-73. 
Zhao, W., Gu, J., Zhang, L., Chen, H. \& Shi, J. (2005). Fabrication of uniform magnetic nanocomposite spheres with a magnetic core/ mesoporous silica shell structure. J. Am. Chem. Soc., 127, 8916-8917. 